\title{
Search for the Standard Model Higgs Boson in Associated Production with W Boson at the Tevatron
}

\author{
by \\ Chun Xu \\ A dissertation submitted in partial fulfillment \\ of the requirements for the degree of \\ Doctor of Philosophy \\ (Physics) \\ in The University of Michigan \\ 2010
}

Doctoral Committee:

Professor Jianming Qian, Chair

Professor Ratindranath Akhoury

Professor Homer A. Neal

Associate Professor Ji Zhu

Assistant Professor Aaron T. Pierce 


\section{ACKNOWLEDGEMENTS}

The work presented in this thesis is done at the $\mathrm{D} \varnothing$ experiment. I would like to thank all the dedicated people in the $\mathrm{D} \varnothing$ collaboration, for their efforts to build the foundation on which a physics analysis can rely.

I would like to express my gratitude to my advisor, Jianming Qian, for his mentoring and support in the past five years. No matter what kind of problem I ran into, I was always able to have valuable and constructive suggestions from him. His physics acumen and attitude towards solving a problem are the best things I can learn from an advisor.

I am deeply indebted to a long list of collaborators: Yuji Enari, Alan Magerkurth, Jeremie Lellouch, Gregorio Bernardi, Nils Huske, Michiel Sanders, Jonas Strandberg, Thomas Gadfort, Dale Johnston, Aurelio Juste, etc. I enjoyed working together with Yuji through the difficult stages of this analysis. I am very grateful for Alan's major contribution to the Matrix Element Method in the analysis. I want to thank everyone working on WH analysis at D $\varnothing$ for their great help. All the comments and suggestions from the Higgs group and the $\mathrm{V}+$ Jets group at D $\varnothing$ experiment are also greatly appreciated.

I would like to give my thanks to Ratindranath Akhoury, Homer Neal, Aaron Pierce, Ji Zhu for serving on my committee. Thanks to Kenneth Herner for correcting grammatical and typographical errors in the thesis. 


\section{TABLE OF CONTENTS}

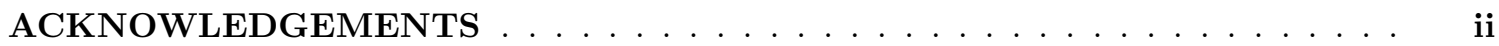

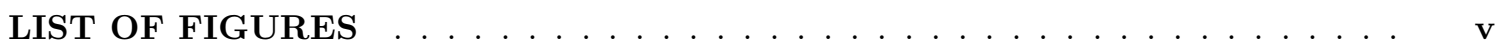

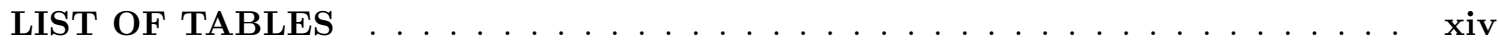

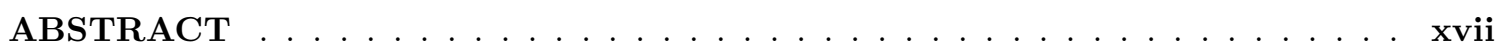

\section{CHAPTER}

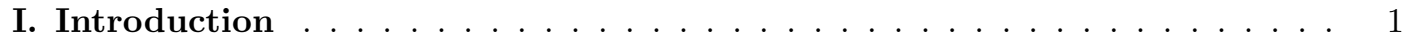

II. The Standard Model Higgs Production and Decay in Hadron Collisions . 4

2.1 The Standard Model, Higgs Mechanism and Higgs Boson . . . . . . . . . . . 4

2.1.1 The Standard Model Theory . . . . . . . . . . . . . . . . . . . 4

2.1.2 Spontaneous Symmetry Breaking and the Higgs Mechanism . . . . 7

2.1.3 The Higgs Boson . . . . . . . . . . . . . . . . . . . . 8

2.2 A Review on the Searches for the Higgs Boson . . . . . . . . . . . . . . . 10

2.2.1 Constraints on the Higgs Boson Mass . . . . . . . . . . . . . . . . 10

2.2.2 Search for the Higgs Boson at the Tevatron . . . . . . . . . . . . 13

2.3 The Higgs Boson Production and Decay at Hadron Colliders . . . . . . . . . 14

2.3.1 Production of the Standard Model Higgs Boson . . . . . . . . . . . 14

2.3.2 Decay of the Standard Model Higgs Boson . . . . . . . . . . . . . 16

2.3.3 Search for $p \bar{p} \rightarrow W^{ \pm} H \rightarrow l \nu b \bar{b}$ at the Tevatron . . . . . . . . . 19

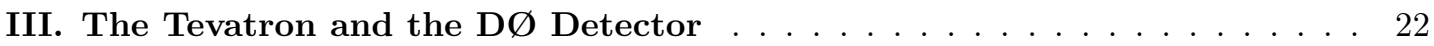

3.1 The Tevatron Accelerator . . . . . . . . . . . . . . . . . . . . 22

3.1.1 An Overview of the Accelerators at the Tevatron . . . . . . . . . 22

3.1 .2 The Tevatron, Cross Section and Luminosity . . . . . . . . . . 28

3.2 The Upgraded D $\varnothing$ Detector . . . . . . . . . . . . . . . . . . . . 30

3.2 .1 Overview of the $\mathrm{D} \emptyset$ Detector . . . . . . . . . . . . 30

3.2 .2 Tracking System . . . . . . . . . . . . . . . . . . . 32

3.2.3 Calorimeter System . . . . . . . . . . . . . . . . . . . . . . 37

3.2 .4 The Muon System . . . . . . . . . . . . . . . . . . . . . . 39

3.2.5 Luminosity Monitor, Trigger System and Data Acquisition System 42

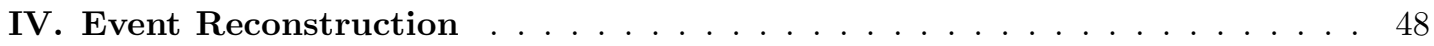

4.1 Offline Event Reconstruction . . . . . . . . . . . . . . . . . . . . . . . . . . 48

4.1.1 Track and Primary Vertex Reconstruction . . . . . . . . . . . . 49

4.1.2 Electromagnetic Object Reconstruction . . . . . . . . . . . . 52 
4.1.3 Jet Reconstruction and Identification . . . . . . . . . . . . . . . . 54

4.1.4 Missing Transverse Energy . . . . . . . . . . . . . . . . 57

4.1.5 Muon Reconstruction and Identification . . . . . . . . . . . . 58

4.2 Corrections to Physics Objects . . . . . . . . . . . . . . . . . 60

4.2.1 Jet Energy Scale, JetSSR and Jet ID Efficiency Corrections . . . . 60

4.2 .2 Lepton ID Efficiency Correction . . . . . . . . . . . . . . . 65

4.2.3 Missing $E_{T}$ Recalculation . . . . . . . . . . . . . . 67

4.3 Identification of b Jets . . . . . . . . . . . . . . . . . . . 67

4.3.1 The DØ Neural Network b-jet Tagger . . . . . . . . . . . . . 67

4.3.2 Tag Rate Function and Taggability . . . . . . . . . . . . . 74

V. The Datasets and Event Selection . . . . . . . . . . . . . . . . . . . . . . . 79

5.1 Data Sample and Simulated Events . . . . . . . . . . . . . . . . 79

5.1.1 Data Sample, Luminosities and Triggers . . . . . . . . . . . . 79

5.1.2 Simulated Event Samples . . . . . . . . . . . . . . . . 85

5.2 The Event Selection Criteria . . . . . . . . . . . . . . . . . . . . 93

$5.2 .1 W$ Boson Identification . . . . . . . . . . . . . . . . . 93

5.2 .2 Jet Selection . . . . . . . . . . . . . . . . . . . . 95

5.2.3 Multijet Background Estimation . . . . . . . . . . . . . 96

5.2.4 Normalization of Simulated Events . . . . . . . . . . . . . . . 98

5.3 The Event Selection of b-jet Tagging . . . . . . . . . . . . . . 100

VI. Multivariate Analysis ........................... 113

6.1 Matrix Element Method ....................... 113

6.1.1 Introduction to the Method ................ 113

6.1.2 Event Probability Density Function . . . . . . . . . . . . . . 114

6.1.3 The Matrix Element Discriminant . . . . . . . . . . . . . 119

6.2 Artificial Neural Network . . . . . . . . . . . . . . . . . . . 122

6.2.1 The Architecture of the Network . . . . . . . . . . . . . . . 122

6.2 .2 The Input Variables . . . . . . . . . . . . . . . . . . 123

6.2.3 The Neural Network Training and the Output . . . . . . . . . . 124

VII. Results of WH Production Search . . . . . . . . . . . . . . . . . . . 144

7.1 Systematic Uncertainties . . . . . . . . . . . . . . . . . . . 144

7.2 Limits on WH Production Cross Sections . . . . . . . . . . . . . . . . . 146

7.2.1 The Limit Calculation Method . . . . . . . . . . . . . . . 151

7.2.2 The Upper Limits on $W H$ Production Cross Section . . . . . . . . 153

7.3 Conclusions ......................... . . 155

BIBLIOGRAPHY . . . . . . . . . . . . . . . . . . . . . . 161 


\section{LIST OF FIGURES}

\section{Figure}

2.1 The Higgs boson couplings to gauge bosons, fermions and itself in the Standard Model. The coupling parameters are respectively $g_{H V V}, g_{H H V V}, g_{H f f}, g_{H H H}$ and

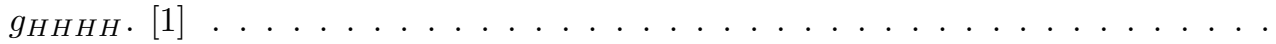

2.2 Constraints on the Higgs boson mass as a function of energy cutoff scale $\Lambda$ [2]. The upper bound is derived from triviality, while the lower bound is from the vacuum stability. The top quark mass and strong coupling constant in the calculation are $m_{t}=175 \pm 6 \mathrm{GeV}$ and $\alpha_{s}\left(M_{Z}\right)=0.118 \pm 0.002$ respectively. . . . . . . . . .

2.3 The $\Delta \chi^{2}=\chi^{2}-\chi_{\min }^{2}$ of the fit to electroweak precision data as a function of Higgs boson mass $m_{H}$. The solid line is the fitted value including all $h i g h-Q^{2}$ data. The shaded band represents theoretical uncertainty from unknown higher order corrections. The vertical bands are the exclusion areas resulted from direct searches for Higgs boson at LEP and the Tevatron. Also shown in the plot are uses of different $\Delta \alpha_{\text {had }}$ values and inclusion of $l o w-Q^{2}$ data. [3] . . . . . . . . .

2.4 The sensitivity of the Higgs boson at the Tevatron. The corresponding integrated luminosities as a function of Higgs boson mass are expected to have exclusion at $95 \%$ C.L., $3 \sigma$ evidence and $5 \sigma$ discovery. The narrow curves are the updated studies in 2003, while the thicker curves are the results of SHWG study in 1999. [4] . . .

2.5 The major production processes of the Standard Model Higgs boson at hadron colliders. [1] (a) gluon-gluon fusion. (b) vector boson fusion. (c) associated production with $W^{ \pm}$and $Z$ bosons. (d) associated production with heavy quarks. . . . . . . .

2.6 The cross sections of the Standard Model Higgs boson production as a function of the Higgs boson mass. Results are for the main production mechanisms at the Tevatron of $\sqrt{s}=1.96 \mathrm{TeV}$. The calculation is valid at NNLO in the QCD expansion for $g g \rightarrow H, q \bar{q} \rightarrow V H$ and $b \bar{b} \rightarrow H$, while it's accurate at NLO for $q q \rightarrow q q H$ and $g g, q q \rightarrow t \bar{t} H \ldots \ldots \ldots \ldots \ldots \ldots$

2.7 The decay width $\Gamma(H)$ as a function of Higgs boson mass in the Standard Model [1]. The data for Standard Model Higgs boson decay is obtained using the program

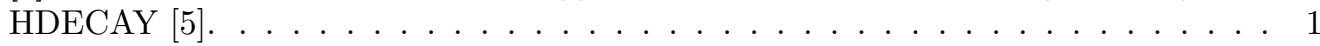

2.8 The branching ratios $B R(H)$ as a function of Higgs boson mass in the Standard Model [1], as calculated using the program HDECAY [5] . . . . . . . . . . . . 19 
2.9 The Higgs production cross section times the branching ratios of main decay modes at the Tevatron as a function of Higgs boson mass. The NNLO cross sections of Higgs boson production are used in the calculation. The branching ratios of Higgs boson decay and $W / Z$ boson decay with single lepton flavor have been taken into

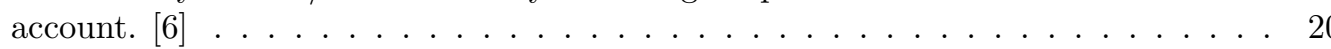

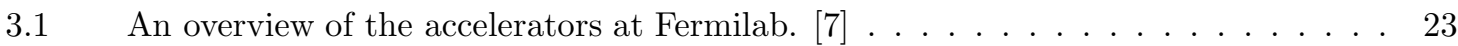

3.2 The basic configuration of the magnetron surface plasma source. [8] . . . . . . 24

3.3 Schematic diagram of a simple two-stage diode voltage multiplier. Each stage of the chain yields $2 V_{0}$ output. The dotted lines represent special addition in the five-stage Fermilab Cockcroft-Walton accelerator. The purpose of this addition is to reduce the ripple while yielding the same output. $[9] \ldots \ldots \ldots \ldots$

3.4 A simplified top view of Alvarez drift tube linac. A RF tank contains $n+1$ resonant cells. Each cell is filled with a bunch of particles. The particles are alternatively focused and defocused using quadrupole magnets embedded within the drift tubes. The bunches of particles are always accelerated in the gap between drift tubes while they are shielded in the drift tubes from the field of RF tank. $[9] \ldots \ldots$. . . . .

3.5 The lithium lens used to collect antiprotons from the secondary particles originated from the target. $[10] \ldots \ldots \ldots \ldots \ldots \ldots$

3.6 Overview of the upgraded D0 detector from inside the Tevatron ring. The forward proton detector is not shown. The details of the central region are shown in Fig. [11] 31

3.7 A side view of the D0 central tracking detectors in the $x-z$ plane. The central tracking detectors include the SMT, CFT and Solenoid outside the beam pipe. The luminosity monitor, preshower detectors and parts of the calorimeters are also shown in the diagram. $[11] \ldots \ldots \ldots \ldots \ldots$

3.8 The layout of the D0 Silicon Microstrip Tracker with its disk and barrel design. [11] 34

3.9 Schematic view of the D0 Central Fiber Tracker. a) A side view of the CFT in $r-z$ plane. It is located between the SMT and the Solenoid. There are 8 layers of concentric barrels labeled as \#1 to \#8 from the innermost the outermost. b) A magnified end view of the barrels in $x-y$ plane. Each barrel contains an axial doublet layer along the beam direction and another steoreo doublet layer at a stereo

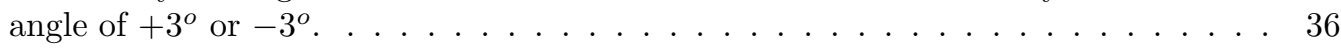

3.10 The magnetic field lines in $y-z$ plane of the DØmagnetic system. The field strengths of both solenoidal and toroidal magnets are in unit of kiloGauss (1 kiloGauss $=0.1$ Tesla), at full current $4749 A$ and $1500 A$ respectively. The field in the central toroid is $\sim 1.8 T$, while it is $\sim 1.9 T$ in the end toroid. [11] . . . . . .

3.11 Schematic view of unit cell for the calorimeter. A unit cell includes the absorber plate and signal board with gaps filled with liquid argon. The absorber plate is made of depleted uranium, while the signal board is copper pad insulated with G10 insulator and coated with highly resistive epoxy. A high voltage of typically $2 \mathrm{kV}$ is applied between the signal board and the absorber plate. The liquid argon gap is $2.3 \mathrm{~mm}$ and takes the electrons about $450 \mathrm{~ns}$ to drift across to the signal board.

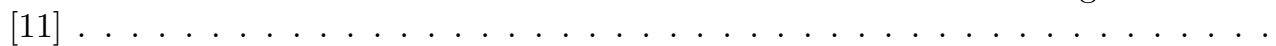


3.12 Isometric view of the central and two end calorimeters. Electromagnetic modules have 4 layers with absorber materials of approximately 1.4,2.0,6.8, $9.8 X_{0}$ in the central and 1.6,2.6,7.9,9.3 $X_{0}$ in the end. In the central region, the hadronic modules have 1.3,1.0,0.76 $\lambda_{0}$ for the fine ones and $3.2 \lambda_{0}$ for the coarse part. In the two end regions, the absorb depths are $4 \times 1.1+4.1 \lambda_{0}$ for the inner hadronic, $4 \times 0.9+4.4$ $\lambda_{0}$ for the middle hadronic and $6.0 \lambda_{0}$ for the outer hadronic. $[11] \ldots \ldots$. . . . .

3.13 Schematic view of a portion of the DØcalorimeters in $r-z$ plane. The CC covers up to $|\eta|=1.1$ detector region, while the EC covers the region of $1.5<|\eta|<4.0$. The ICR region of $1.1<|e t a|<1.4$ is covered by ICD. [11] . . . . . . . . . .

3.14 Diagrams of the DØRun II Luminosity Monitor. a) $r-\phi$ view of the monitors. The wedge shaped are the the scintillation tiles, on which the small circles are the corresponding photomultiplier tubes. b) $r-z$ view of the monitors. The two monitors are positioned at $z= \pm 140 \mathrm{~cm}$ along the beamline. [12] . . . . . . .

3.15 The total integrated luminosity. The lower curve is the luminosity recorded by the DØdetector, while the upper curve is the luminosity delivered by the Tevatron. . .

3.16 An overview of the data flow in the DØtrigger and data acquisition systems. [11] . 45

3.17 Information flow of the DØLevel 1 and Level 2 trigger systems. [11] . . . . . . 46

3.18 Schematic view of the data flow in the DØL3DAQ system. [11] $\ldots \ldots \ldots \ldots$

4.1 a) $p_{T}$ spectrum of tracks from the hard scattering physics data events and the simulated minimum bias events. b) Vertex probability from the hard scattering physics data events and the simulated minimum bias events. $[13] \ldots \ldots \ldots$

4.2 a) $\chi^{2}$ test on H-matrix with seven shower shape variables. b) Electron likelihood discriminant distribution. The real electron and fake electron events are both shown in the plots. $[14] \ldots \ldots \ldots \ldots \ldots \ldots$

4.3 An illustration of the sensitivity in the seed-based cone jet clustering. In case there is soft gluon radiated by either of the two independent jets, the two jets could be treated as single jet in the seed-based cone jet clustering. [15] . . . . . . . . .

4.4 Offset energy as a function of jet detector eta $\eta_{\text {det }}$. a) Energy offset determined in Run IIa datasets. b) Energy offset determined in Run IIb dataset. Its dependence on number of primary vertices is also shown in the plots. . . . . . . . . . .

4.5 Relative MPF Correction using $\gamma+j e t$ in data events a function of jet detector eta $\eta_{\text {det }}$. a) with 0.7 jet cone Run IIa datasets. b) With 0.5 jet cone in Run IIb datasets. Its dependence on the energy is also shown in the plots. . . . . . . . .

4.6 Showering correction using $\gamma+$ jet events as a function of jet energy in Run IIa. a) With 0.5 jet cone. b) With 0.7 jet cone. Its dependence on detector eta is also shown in the plots. . . . . . . . . . . . . . . . .

4.7 The total JES correction as a function of jet pseudorapidity using $\gamma+$ jet events in Run IIb datasets. Different curves correspond to different measured jet transverse energies. . . . . . . . . . . . . . . . . . . 
4.8 The smearing factor as a function of $p_{T}^{\gamma}$ applied to simulated events. The curves represent different detector regions: CC (blue), ICR (red) and EC (green). The dashed curves reflect the statistical uncertainties only. . . . . . . . . . . . . . .

4.9 The difference in jet energy scale correction between data and simulated events, as a function of $p_{T}^{\gamma}$. Shown in the plot is for CC detector region only. The yellow band represents the statistical errors. . . . . . . . . . . . . . .

4.10 The turn-on curves of jet identification efficiencies as a function of jet $p_{T}$. a) Data. b) MC. The dashed curves represent statistical errors only. . . . . . . . . . . . .

4.11 The scale factor for correcting jet identification efficiency difference between data and simulated events, as a function of $\left|\eta_{D}\right|$ binned by 0.1 . The scale factor is determined using the jets in the reconstruction efficiency plateau region and Run IIb datasets only. $[16] \ldots \ldots \ldots \ldots$. . . . . . . . . . . . . .

4.12 The muon smearing parameters as a function of muon $p_{T}$ for events where both muons have SMT hits and $\left|\eta_{C F T}\right|<1.6$. The dashed lines represent the $1 \sigma$ uncertainties of the two parameterizations. $[17] \ldots \ldots \ldots \ldots$

4.13 The correction factor of medium $n s e g=3$ muon identification efficiency as a function of $\eta$. Muons in the hole regions are not considered. . . . . . . . . . . .

4.14 The correction factor of medium tracking efficiency as a function of CFT detector

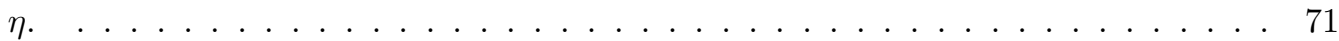

4.15 The correction factors of muon isolation efficiencies as a function of muon $p_{T}$. . . 72

4.16 The correction factors of muon isolation efficiencies as a function of number of jets with $p_{T}>15 \mathrm{GeV} \ldots \ldots \ldots \ldots \ldots \ldots$

4.17 A schematic view of b hadron production and decay in $p \bar{p}$ collisions. $L$ is the $\mathrm{b}$ hadron decay length, while $d_{0}$ is the impact parameter for one of the tracks. . . .

4.18 The b-jet tagging efficiency versus the tagging fate rate for the 12 operation points of the DØNN b-jet tagger. The blue dots are determined on Run IIb datasets while the red dots are determined on Run IIa datasets. The error represents the full statistical and systematic uncertainty with which we know the data efficiency and fake rate for the samples on a per jet basis. $[18] \ldots \ldots \ldots$

4.19 b-tagging efficiency as a function of $p_{T}$ or $\eta$ for the Tight operation point of the NN b-jet tagger. It is determined on Run IIb datasets (red for MC and green for data) in the CC, ICR and EC region respectively. The TRFb on data is obtained by multiplying the $\mathrm{MC}$ b-jet tagging efficiency by the scale factor. The dotted black lines are the fit errors dominated by the scale factor fit errors. $[18] \ldots \ldots$. . .

$4.20 \mathrm{c}$ jet tagging efficiency as a function of $p_{T}$ or $\eta$ for the Tight operation point of the $\mathrm{NN}$ tagger. It is determined on Run IIb datasets (red for MC and green for data) in the CC, ICR and EC region respectively. The TRFc on data is obtained by multiplying the b-jet tagging efficiency by the $\mathrm{c} / \mathrm{b}$ scale factor. The dotted black lines are the fit errors dominated by the scale factor fit errors. [18] . . . . . . . 77 
4.21 Closure test for p20 Jet taggability scale factors for electron and muon channel (a) Electron $p_{T}$ dependence, (b) Electron $\eta$ dependence, (c) Muon $p_{T}$ dependence and (d) Muon $\eta$ dependence.

5.1 Kinematic distributions for CC+EC electrons of the $W+2$ jet event sample (Run IIb data set): a) energy; b) transverse momentum; c) $\eta$; d) $\varphi$. The simulation is normalized to the integrated luminosity of the data sample using the expected cross sections (absolute normalization) except for the $W+$ jets sample which is normalized to data on the "pre-tag sample", taking into account all the other backgrounds. . . . . . . . . . . . . . . . . . . . . 103

5.2 Kinematic distributions for muons of the $W+2$ jet event sample (Run IIb data set): a) energy; b) transverse momentum; c) $\eta$; d) $\varphi$. The simulation is normalized to the integrated luminosity of the data sample using the expected cross sections (absolute normalization) except for the $W+$ jets sample which is normalized to data on the "pre-tag sample", taking into account all the other backgrounds. . . . 104

5.3 Distribution in the $W+2$ jets sample of the (a) lepton momentum, (b) the transverse $W$ mass, (c) the $H_{T}$ variable and (d) missing transverse energy compared to the simulated expectation in the $W+2$ jet event sample. The simulation is normalized to the integrated luminosity of the data sample using the expected cross sections (absolute normalization) except for the $W+$ jets sample which is normalized on the "untagged sample" to the data, taking into account all the other backgrounds. . . . . . . . . . . . . . . . . . 105

$5.4 \quad$ Distribution in the $W+2$ jets sample of the (a) $p_{T}$ of the leading and (b) next to leading jet, (c) of the distance in the $\eta-\varphi$ plane between the two jets and (d) of the dijet mass (d) between the two jets in the $W+2$ jet sample compared with the simulated expectation. The simulation is normalized to the integrated luminosity of the data sample using the expected cross sections (absolute normalization) except for the $W+$ jets sample which is normalized on the "untagged sample" to the data, taking into account all the other backgrounds. . . . . . . . . . . . . . 106

5.5 The $\epsilon_{W}$ and $\epsilon_{Q C D}$ as a function of muon $p_{T}$. The upper blue and lower red solid lines represent $\epsilon_{W}$ and $\epsilon_{Q C D}$ determined from Run IIb datasets, while the upper blue and lower red dotted lines are determined from Run IIa datasets. Note that the $W$ contributions to $\epsilon_{Q C D}$ is not subtracted in Run IIa calculations. . . . . . . . 107

5.6 (a) W transverse mass for loose electron on data (black) as QCD sample and WH $\mathrm{MC}$ (red) as real $\mathrm{W}$ sample. (b) 2D ratio plot of data / WH MC on W transverse mass vs Missing Et. Normalization of WH MC is same as in Fig. (a). QCD events distribute low $\mathbb{E}_{T}$ and low $M_{W}^{T}$ which can be eliminated by triangle cut of $M_{W}^{T}>-0.5 E_{T}+40 G e V$ as shown in black line. WH MC is normalized on W peak. 107

5.7 Reweighting functions on the jet angular distributions of simulated $W / Z+$ jets events. (a) Leading jet $\eta$. (b) $2^{\text {nd }}$ Leading jet $\eta$. (c) $\Delta \eta$ (jet 1 , jet 2$)$. (d) $\Delta \phi($ jet 1 , jet 2 ). 108

5.8 Shape uncertainty on the dijet mass in $W j j$ and $W b b$ events: a) uncertainty originating from the $\eta, \varphi, \delta-\eta, \delta-\varphi$ reweighting; b) additional uncertainty on the shape as obtained by a fit to the ratio of data to the reweighted simulation to the data; the fitted result is also shown; the actual uncertainty is the fit to which is conservatively added another $+5 \%$ uncertainty. . . . . . . . . . . . . . 108 
5.9 Kinematic distributions in the $W+2 j$ ets sample with exactly one jet b-tagged. (a) $p_{T}$ of the leading jet. (b) $p_{T}$ of the second leading jet. (c) $\Delta R$ between the two leading jets. (d) invariant mass of the leading two jets. The dots represent the distribution of data events, which are compared to the solid line representing the sum of the background events. The simulated events are fully normalized and corrected as discussed previous sections. The expected contribution of the Standard Model WH events with $m_{H}=115 \mathrm{GeV}$ shown in the plot scaled up by a factor of 10.109

5.10 Kinematic distributions in the $W+2 j e t s$ sample with 2 jet b-tagged. (a) $p_{T}$ of the leading jet. (b) $p_{T}$ of the second leading jet. (c) $\Delta R$ between the two leading jets. (d) invariant mass of the leading two jets. The dots represent the distribution of data events, which are compared to the solid line representing the sum of the background events. The simulated events are fully normalized and corrected as discussed previous sections. The expected contribution of the Standard Model WH events with $m_{H}=115 \mathrm{GeV}$ shown in the plot scaled up by a factor of 10 . . . . . 110

5.11 The dijet invariant mass distributions (linear scale) of events with single or double b-tagged jets. a) $W+2$ jet events with exactly one jet tagged as Tight b jet. b) $W+3 j$ jet events with exactly one jet tagged as Tight b jet. c) $W+2$ jet events with at least two jets tagged as oldLoose b jets. d) $W+3$ jet events with at least two jets tagged as oldLoose $\mathrm{b}$ jets. The simulated processes are normalized to the integrated luminosity of the data sample using the expected cross sections (absolute normalization) except for the $W+$ jets sample which is normalized on the "untagged sample" to the data, taking into account all the other backgrounds. The backgrounds labeled as "other" in the figure are dominated by single-top production. Also shown is the contribution expected for standard model $W H$ production with $m_{H}=115 \mathrm{GeV}$, multiplied by a factor $10 \ldots \ldots \ldots \ldots 11$

5.12 The dijet invariant mass distributions (logarithmic scale) of events with single or double b-tagged jets. a) $W+2$ jet events with exactly one jet tagged as Tight $\mathrm{b}$ jet. b) $W+3$ jet events with exactly one jet tagged as Tight $\mathrm{b}$ jet. c) $W+$ 2 jet events with at least two jets tagged as oldLoose b jets. d) $W+3$ jet events with at least two jets tagged as oldLoose $b$ jets. The simulated processes are normalized to the integrated luminosity of the data sample using the expected cross sections (absolute normalization) except for the $W+$ jets sample which is normalized on the "untagged sample" to the data, taking into account all the other backgrounds. The backgrounds labeled as "other" in the figure are dominated by single-top production. Also shown is the contribution expected for standard model $W H$ production with $m_{H}=115 \mathrm{GeV}$, multiplied by a factor $10 . \ldots 112$

6.1 The corresponding Feynman diagrams of the leading-order matrix elements used in the calculation of event probability density functions. Upper row: $u d \rightarrow t b, u b \rightarrow t d$; Middle row: $u d \rightarrow W b b, s g \rightarrow W c g, u d \rightarrow W g g$. Lower row: $u u \rightarrow W W, u d \rightarrow W Z$,

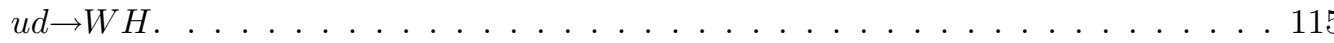

6.2 Energy difference between a reconstructed jet and its matched parton for three types of jets for all detector regions and all jet energies. . . . . . . . . . . . . . 116

6.3 Result of the two-dimensional grid optimization for the constants $C_{W b b}$ and $C_{W c g}$ in the $e+$ jets channel for single and double tagged events. The result shown is for a Higgs mass of $115 \mathrm{GeV}$. . . . . . . . . . . . . . . . . . . . . 121 
6.4 Result of the two-dimensional grid optimization for the constants $C_{W b b}$ and $C_{W c g}$ in the $\mu+$ jets channel for single and double tagged events. The result shown is for a Higgs mass of $115 \mathrm{GeV} \ldots \ldots \ldots \ldots$. . . . . . . . . . . . . . . .

6.5 Distributions of the Matrix Element discriminant. The data events are compared to the simulated events for both single and double b-tagged jets. a) ME discriminant for the single b-tagged events. b) ME discriminant for the double b-tagged events. c) Log scale of figure (a). d) Log scale of figure (b). The simulation is normalized to the integrated luminosity of the data sample using the expected cross sections (absolute normalization) except for the $W+$ jets sample which is normalized on the "pre-tag sample" to the data, taking into account of all the other backgrounds. The $W H$ expected contribution which is scaled by a factor of 10 is peaking at high values of the ME discriminant as shown in c) and $d)$. . . . . . . . . . . . 126

6.6 Discriminating power represented by the neural network output difference for each input variable in a nine-input neural network to separate $W H$ signal events from from $W b b$ background events. . . . . . . . . . . . . . . . . . . . . . 127

6.7 Distributions of the input variables for the neural network, of the $W+2$ jet electron $(\mathrm{CC}+\mathrm{EC})$ analysis with the Run IIb data set before $b$-tagging: a) $p_{T}$ of leading jet, b) $p_{T}$ of sub-leading jet, c) $p_{T}$ of $e-\mathbb{E}_{T}$ system, d) dijet invariant mass. . . . . . 128

6.8 Distributions of the input variables for the neural network, of the $W+2$ jet muon analysis with the Run IIb data set before $b$-tagging: a) $p_{T}$ of leading jet, b) $p_{T}$ of sub-leading jet, c) $p_{T}$ of $\mu$ - $E_{T}$ system, d) dijet invariant mass. . . . . . . . .

6.9 Distributions of the input variables for the neural network and the neural network output of the $W+2$ jet electron (CC+EC) analysis with the Run IIb data set, before $b$-tagging: a) $p_{T}$ of dijet system, b) $\Delta \phi$ between two leading jets, c) $\Delta R$ between two leading jets, d) neural network output (using the $m_{H}=115 \mathrm{GeV}$, double $b$-tag neural network). . . . . . . . . . . . . . . . . . 130

6.10 Distributions of the input variables for the neural network and the neural network output of the $W+2$ jet muon analysis with the Run IIb data set, before $b$-tagging: a) $p_{T}$ of dijet system, b) $\Delta \phi$ between two leading jets, c) $\Delta R$ between two leading jets, d) neural network output (using the $m_{H}=115 \mathrm{GeV}$, double $b$-tag neural

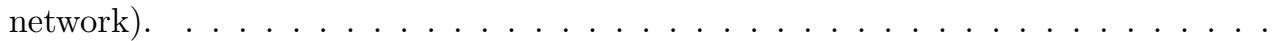

6.11 Distributions of the input variables for the neural network of the $W+2$ jet electron $(\mathrm{CC}+\mathrm{EC})$ analysis with the Run IIb data set, for events with a single $b$-tag (exclusive): a) $p_{T}$ of leading jet, b) $p_{T}$ of sub-leading jet, c) $p_{T}$ of $e-E_{T}$ system, d) dijet invariant mass. . . . . . . . . . . . . . . . . 132

6.12 Distributions of the input variables for the neural network of the $W+2$ jet muon analysis with the Run IIb data set, for events with a single $b$-tag (exclusive): a) $p_{T}$ of leading jet, b) $p_{T}$ of sub-leading jet, c) $p_{T}$ of $\mu$ - $\mathbb{E}_{T}$ system, d) dijet invariant mass.133

6.13 Distributions of the input variables for the neural network and the neural network output of the $W+2$ jet electron $(\mathrm{CC}+\mathrm{EC})$ analysis with the Run IIb data set, for events with a single $b$-tag (exclusive): $b$-tagging: a) $p_{T}$ of dijet system, b) $\Delta \phi$ between two leading jets, c) $\Delta R$ between two leading jets, d) neural network output (using the $m_{H}=115 \mathrm{GeV}$, single $b$-tag neural network) . . . . . . . . . . . 134 
6.14 Distributions of the input variables for the neural network and the neural network output of the $W+2$ jet muon analysis with the Run IIb data set, for events with a single $b$-tag (exclusive): $b$-tagging: a) $p_{T}$ of dijet system, b) $\Delta \phi$ between two leading jets, c) $\Delta R$ between two leading jets, d) neural network output (using the $m_{H}=115 \mathrm{GeV}$, single $b$-tag neural network) . . . . . . . . . . 135

6.15 Distributions of the input variables for the neural network of the $W+2$ jet electron $(\mathrm{CC}+\mathrm{EC})$ analysis with the Run IIb data set, for events with a double $b$-tag: a) $p_{T}$ of leading jet, b) $p_{T}$ of sub-leading jet, c) $p_{T}$ of $e-\mathbb{E}_{T}$ system, d) dijet invariant mass. 136

6.16 Distributions of the input variables for the neural network of the $W+2$ jet muon analysis with the Run IIb data set, for events with a double $b$-tag: a) $p_{T}$ of leading jet, b) $p_{T}$ of sub-leading jet, c) $p_{T}$ of $\mu$ - $\mathbb{T}_{T}$ system, d) dijet invariant mass. . . . . 137

6.17 Distributions of the input variables for the neural network and the neural network output of the $W+2$ jet electron $(\mathrm{CC}+\mathrm{EC})$ analysis with the Run IIb data set, for events with a double $b$-tag: $b$-tagging: a) $p_{T}$ of dijet system, b) $\Delta \phi$ between two leading jets, c) $\Delta R$ between two leading jets, d) neural network output (using the $m_{H}=115 \mathrm{GeV}$, double $b$-tag neural network) . . . . . . . . . . .

6.18 Distributions of the input variables for the neural network and the neural network output of the $W+2$ jet muon analysis with the Run IIb data set, for events with a double $b$-tag: $b$-tagging: a) $p_{T}$ of dijet system, b) $\Delta \phi$ between two leading jets, c) $\Delta R$ between two leading jets, d) neural network output (using the $m_{H}=115$ $\mathrm{GeV}$, double $b$-tag neural network) . . . . . . . . . . . . . . . . 139

6.19 Distributions (Log-y) of the input variables for the neural network and the neural network output, of the $W+2$ jet electron analysis with the Run IIb data set, for events with a double $b$-tag: $b$-tagging: a) $p_{T}$ of dijet system, b) $\Delta \phi$ between two leading jets, c) $\Delta R$ between two leading jets, d) neural network output (using the $m_{H}=115 \mathrm{GeV}$, double $b$-tag neural network). . . . . . . . . . . . . 140

6.20 Distributions (Log-y) of the input variables for the neural network and the neural network output, of the $W+2$ jet muon analysis with the Run IIb data set, for events with a double $b$-tag: $b$-tagging: a) $p_{T}$ of dijet system, b) $\Delta \phi$ between two leading jets, c) $\Delta R$ between two leading jets, d) neural network output (using the $m_{H}=115 \mathrm{GeV}$, double $b$-tag neural network) . . . . . . . . . . . . . 141

6.21 Distributions (with linear vertical scale) of the neural network output compared with the simulated expectation. a) before $b$-tagging for the single-tag NN. b) before $b$-tagging for the double-tag NN. c) in the single $b$-tagged sample for the single-tag NN. d) in the double $b$-tagged sample for the double-tag NN ; The simulation is normalized to the integrated luminosity of the data sample using the expected cross sections (absolute normalization) except for the $W+$ jets sample which is normalized on the "pre-tag sample" to the data, taking into account all the other backgrounds. The $W H$ expected contribution for $m_{H}=115 \mathrm{GeV}$, multiplied by a factor 10, is peaking at high values of the $\mathrm{NN}$ output as shown in c) and d). . . . 142 
6.22 Distributions (with logarithmic vertical scale) of the neural network output compared with the simulated expectation. a) before $b$-tagging for the single-tag NN. b) before $b$-tagging for the double-tag NN. c) in the single $b$-tagged sample for the single-tag NN. d) in the double $b$-tagged sample for the double-tag NN ; The simulation is normalized to the integrated luminosity of the data sample using the expected cross sections (absolute normalization) except for the $W+$ jets sample which is normalized on the "pre-tag sample" to the data, taking into account all the other backgrounds. The $W H$ expected contribution for $m_{H}=115 \mathrm{GeV}$, multiplied by a factor 10, is peaking at high values of the $\mathrm{NN}$ output as shown in c)

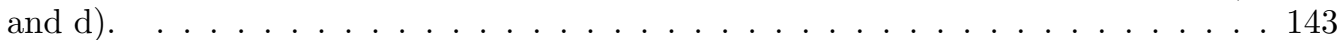

7.1 The results of calculation on the upper limits of $W H$ production cross section $\sigma(p \bar{p} \rightarrow W H) \times B R(H \rightarrow b \bar{b})$. The $2.7 \mathrm{fb}^{-1}$ of Run IIa and RunIIb datasets with the 16 event sub-samples are combined in the plots. a) The log-likelihood ratios as a function of the Higgs boson mass $m_{H}$. The dashed lines represent for $L L R_{b}$ (upper line) and $L L R_{s+b}$ (lower line), while the solid line represents $L L R_{o b s}$. The green band (inner region) and yellow band region (extended from the inner to the outermost) stand for the $\pm 1 \sigma$ and $\pm 2 \sigma$ standard deviations. b) The ratios of the upper limits on the production cross sections to those predicted by the Standard Model for $\sigma(p \bar{p} \rightarrow W H) \times B R(H \rightarrow b \bar{b})$, as a function of $m_{H}$. The solid line and dashed lines represent the observed and expected limits at $95 \%$ C.L. respectively. The straight line at ratio $=1.0$ indicates the Standard Model prediction. . . . . . . 159

7.2 The ratios of $95 \%$ C.L. cross section limit on the Standard Model Higgs production to the Standard Model expectation, as a function of $m_{H}$. The solid and dashed lines represent the observed and expected limits respectively, while the green and yellow bands stand for $1 \sigma$ and $2 \sigma$ standard deviations. The searches for $W H / Z H / H$, with $H \rightarrow b \bar{b} / W^{+} W^{-} / \gamma \gamma / \tau^{+} \tau^{-}$final states, using $0.9-4.2 \mathrm{fb}^{-1}$ of data collected at the DØdetector have been combined in this plot. [19] . . . . . . . . . . . . . 160

7.3 Observed and expected upper limits on the Standard Model Higgs production at the Tevatron as a function of the Higgs boson mass. The limits are expressed as the constraints on the ratio of experimental production cross section to the one predicted by the Standard Model. The solid curve represents the observed ratio, while the dotted is the expected (background-only hypothesis) ratio. The limits are calculated with a Bayesian method at $95 \%$ C.L.. The shaded bands indicate fluctuation regions of the limits at $68 \%$ and $95 \%$ probability respectively in the absence of signal. $[20] \ldots \ldots$. . . . . . . . . . . . . . . . . 160 


\section{LIST OF TABLES}

\section{$\underline{\text { Table }}$}

2.1 List of leptons and quarks in the Standard Model . . . . . . . . . . . . . . . . 5

2.2 List of gauge bosons in the Standard Model . . . . . . . . . . . . . . . . 5

4.1 Overview of the definitions of muon type and quality . [17] . . . . . . . . . . . . . 59

4.2 NN parameters. $[18]$. . . . . . . . . . . . . . . . . . . . . . . . 72

4.3 The Neural Network input variables ranked in order of b-jet discrimination power.

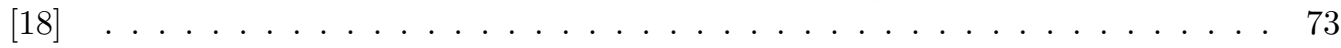

4.4 The operation points of the DØNN b-jet tagger. [18] . . . . . . . . . . . . . . . 74

5.1 Run IIa and Run IIb data samples used in the analysis. . . . . . . . . . . . . . . 80

5.2 Recorded integrated luminosities for the datasets in EM channel. The datasets correspond to different trigger list versions, which are used in different periods of DØdata taking. The triggers in the table are unprescaled triggers. . . . . . . .

5.3 Recorded integrated luminosities for the datasets in MU channel. The datasets correspond to different trigger list versions, which are used in different periods of DØdata taking. The triggers in the table are unprescaled triggers. . . . . . . . . . 82

5.4 EM+JET triggers through trigger versions v8 - v14 in the Run IIa datasets. . . . . 82

5.5 Single EM triggers through trigger versions v8 - v14 in the Run IIa datasets. $\quad$. . . 83

5.6 Single EM triggers through trigger versions v15-v16 in the Run IIb datasets. . . . . 84

5.7 List of Single MU triggers used in the trigger study on Run IIa datasets. . . . . . . 85

5.8 The simulated samples of signal events $p \bar{p} \rightarrow W+H \rightarrow l \nu+b \bar{b}$ and $p \bar{p} \rightarrow Z+H \rightarrow$ $l l+b \bar{b}$. The MC event generators, number of generated events and cross sections are shown in the table for Run IIa and Run IIb respectively. The branching ratios used in the calculation is for only one lepton flavor. . . . . . . . . . . . . . . . 88

5.9 The simulated samples of the diboson processes $W W, W Z$ and $Z Z$. The MC event generators, number of generated events and cross sections are shown in the table for Run IIa and Run IIb respectively. A combination of several exclusive channels is used for Run IIa, while the inclusive samples are used for Run IIb. . . . . . . . . 
5.10 The simulated samples of top pair and electroweak single top productions. The $\mathrm{MC}$ event generators, number of generated events and cross sections are shown in the table for Run IIa and Run IIb respectively. The K factor for $t \bar{t}$ scales the $t \bar{t}$ cross section to $6.8 \mathrm{pb}$. No $\mathrm{K}$ factor is given for single top since the cross section given is already at NLO . . . . . . . . . . . . . . . . . .

5.11 The simulated samples of $W+j e t s$ and $Z+j e t s$ for Run IIa. The MC event generators, number of generated events and cross sections are shown in the table. The samples are generated in bins of light parton multiplicity. Each bin is considered exclusive with a certain number of partons, except that the last bin is inclusive with more partons. The light partons are required to have $p_{T}>8 \mathrm{GeV}$ and $|\eta|<5$. The $l$ is inclusive to be $e, \mu$ and $\tau \ldots \ldots \ldots \ldots \ldots$

5.12 The simulated samples of $W+$ jets and $Z+$ jets for Run IIb. The MC event generators, number of generated events and cross sections are shown in the table. The samples are generated in bins of light parton multiplicity. Each bin is considered exclusive with a certain number of partons, except that the last bin is inclusive with more partons. The light partons are required to have $p_{T}>8 \mathrm{GeV}$ and $|\eta|<5$. The $l$ is inclusive to be $e, \mu$ and $\tau \ldots \ldots \ldots \ldots \ldots$

5.13 Fake rate for $\mathrm{CC}$ and $\mathrm{EC}$ electron channel at $\mathrm{pT}=30 \mathrm{GeV}$ for $\mathrm{v} 8$-v15 trigger lists. Errors are statistical only. An additional 15\% systematic errors on these parameters is considered when estimating the total uncertainty on the QCD background. . . .

5.14 The scale factor $S F$ applied to $W / Z+j e t s$ samples. (errors are statistical only, the uncorrelated systematic uncertainties between the $e$ and $\mu$ determination is greater than $10 \%) . \ldots \ldots \ldots \ldots \ldots \ldots$

5.15 The HF factor determined with b-jet tagging operation points VeryTight, Tight and oldLoose on different datasets. (errors are statistical only, the uncorrelated systematic uncertainties between the $e$ and $\mu$ determination is greater than $10 \%$ ).

5.16 Summary table for the $W+2,3$ jet final states. Observed events in data are compared to the expected number of $W+$ jet events before tagging, with exactly one tight $b$-tagged jet, and with exactly 2 loose $b$-tagged jets. First three columns are for the $W+2$ jet channel, the last three columns for the $W+3$ jet channel. Expectation originates from the simulation of $W H$ and $Z H$ (with $m_{H}=115 \mathrm{GeV}$ ), dibosons ( $W W, W Z, Z Z$, labeled $W Z$ in the table), $W b \bar{b}$ production, top production $(t \bar{t}$ and single-top), multijet background and " $W+$ jet" production, which contains light and $c$ quarks. All $Z$ processes are fully simulated, and included in the corresponding $W$ categories. The processes $W(Z) b \bar{b}$ and $W(Z)+$ light and/or $c$ jets are counted separately. "n.t.d." stands for "normalized to data". The uncertainties given include statistics and systematics. . . . . . . . . . . . . . . . . 102

6.1 For three of the background processes in the analysis, $t \bar{t} \rightarrow \ell+$ jets, $t \bar{t} \rightarrow \ell \ell$ and multijet, there is no dedicated matrix element. Using a $\chi^{2}$ we determined the background sample with a matrix element which is most similar to each of the three. For the purpose of determining the constants in the background probability density function $t \bar{t} \rightarrow \ell+$ jets and $t \bar{t} \rightarrow \ell \ell$ are treated as $t b$ and multijet events are treated as $W+$ jets. . . . . . . . . . . . . . . . . . . 12 
6.2 Background fractions chosen for each analysis channel. The $C_{W b b}, C_{W c g}$ and $C_{W g g}$ constants are obtained from an optimization for the best expected limit on the $W H$ cross section. The optimization is done separately for each Higgs mass, and we use an average over the constants for the various Higgs masses in each of the four channels. . . . . . . . . . . . . . . . . . . . . . . . . . . 12

7.1 Sources of the systematic uncertainties of the sixteen event samples. The naming conventions is explained in the Table 7.2. The terms labeled with $\times$ means they are associated. Those sources with names starting with $B \mathrm{kgd}$ apply only to the background, while the other apply to both the background and signal, except for the last error of the table is only for $W H$.

7.2 The naming convention of the systematic uncertainties in Tab. 7.1. Those sources with names starting with $B k g d$ apply only to the background, while the other apply to both the background and signal, except for the last entry of the table is only for $W H$.

7.3 Systematic uncertainties (\%) for different physics processes containing electron in the final states with single jet b-tagged. The total uncertainties are also summarized in the table. . . . . . . . . . . . . . . . . . . . . . 149

7.4 Systematic uncertainties (\%) for different physics processes containing electron in the final states with double jet b-tagged. The total uncertainties are also summarized in the table. . . . . . . . . . . . . . . . . . . . 149

7.5 Systematic uncertainties (\%) for different physics processes containing muon in the final states with single jet b-tagged. The total uncertainties are also summarized in the table. . . . . . . . . . . . . . . . . . 150

7.6 Systematic uncertainties (\%) for different physics processes containing muon in the final states with double jet b-tagged. The total uncertainties are also summarized in the table. . . . . . . . . . . . . . . . . . . . . . . . . . . . .

7.7 The ratios of the expected (observed) production cross section limits to the Standard Model prediction, where the cross section is $\sigma(p \bar{p} \rightarrow W H) \times B R(H \rightarrow b \bar{b})$ with the Higgs boson mass $m_{H}=115 \mathrm{GeV}$. The electron and muon, single and double b-tagged have been combined for all the numbers in the table. The $1.0 \mathrm{fb}^{-1}$ Run IIa results (March 2007) are the previous cut-based analysis without multivariate analysis techniques. . . . . . . . . . . . . . . . . . 154

7.8 The ratios of $95 \%$ C.L. expected and observed limits on $\sigma(p \bar{p} \rightarrow W H) \times B(H \rightarrow b \bar{b})$, to their corresponding Standard Model prediction, as a function of the Higgs boson

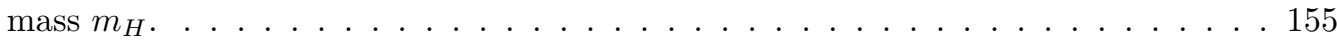




\begin{abstract}
A search for the Standard Model Higgs boson in proton-antiproton collisions with center-of-mass energy $1.96 \mathrm{TeV}$ at the Tevatron is presented in this dissertation. The process of interest is the associated production of $\mathrm{W}$ boson and Higgs boson, with the $\mathrm{W}$ boson decaying leptonically and the Higgs boson decaying into a pair of bottom quarks. The dataset in the analysis is accumulated by the $\mathrm{D} \varnothing$ detector from April 2002 to April 2008 and corresponding to an integrated luminosity of $2.7 \mathrm{fb}^{-1}$. The events are reconstructed and selected following the criteria of an isolated lepton, missing transverse energy and two jets. The DØ Neural Network b-jet identification algorithm is further used to discriminate $b$ jets from light jets. A multivariate analysis combining Matrix Element and Neural Network methods is explored to improve the Higgs boson signal significance. No evidence of the Higgs boson is observed in this analysis. In consequence, an observed (expected) limit on the ratio of $\sigma(p \bar{p} \rightarrow \mathrm{WH})$ $\times \mathrm{Br}(\mathrm{H} \rightarrow b \bar{b})$ to the Standard Model prediction is set to be 6.7 (6.4) at 95\% C.L. for the Higgs boson with a mass of $115 \mathrm{GeV}$.
\end{abstract}




\section{CHAPTER I}

\section{Introduction}

The Standard Model, as a quantum field theory in particle physics, is developed to describe the elementary particles as well as their interactions. It is well established as a unified framework to explore and understand the matter and forces in our universe. The theory defines a group of fundamental particles which are not able to be further divided into smaller ones. A subset of these particles, known as fermions with spin equal to $1 / 2$, is considered to make up the matter in the world. The other subset of the particles, known as bosons with spin equal to 1, is responsible for mediating forces among these particles.

The model turned out to be a great success both theoretically and experimentally over the past century. It is a gauge theory with symmetry group $S U_{C}(3) \times$ $S U_{L}(2) \times U_{Y}(1)$, which incorporates three of the known fundamental interactions: strong force, weak force and electromagnetic force. The theory precisely interprets that the fermions such as leptons and quarks interact with each other by exchanging the gauge bosons. Nearly all the particles in the Standard Model have been observed in various particle physics experiments. Over half of these particles are actually first predicted by the model and then discovered experimentally. The characteristics of the particles and their interactions are consistent with the theoretical predictions. 
However, there is a mysterious particle, called the Higgs boson, remaining to be discovered in the Standard Model. As a massive boson without intrinsic spin, it is introduced in theory to explain the mass origins of all other particles. The way in which all massive particles acquire their masses is called the Higgs mechanism. In this mechanism, an extra field, called the Higgs field, is introduced to simultaneously break the symmetry of the gauge fields. The Higgs field interacts with these gauge fields and generates the masses of the vector bosons, while it can give the fermion masses through the Yukawa interaction with fermionic fields. The Higgs field also interacts with itself and predicts a massive scalar boson named as Higgs boson.

Search for the Higgs boson has become the center of interest in the recent experiments, particularly at the Tevatron proton-antiproton collider at Fermilab. The mass of the Higgs boson itself is not yet predicted, although the interactions of fields give masses to other particles. It requires very high energy to produce and is unstable with short lifetime. In the past decades, there have been indirect and direct searches for the Higgs boson in several high energy physics experiments. The direct search of LEP experiments at CERN excludes the existence of Higgs boson with mass less than $114 \mathrm{GeV}$ at $95 \%$ C.L. [3]. For the time being, the Tevatron experiments are the only ones in the world capable of directly searching for the Higgs boson.

The dominant process for producing the Higgs boson at the Tevatron is the gluongluon fusion mechanism. For mass below $135 \mathrm{GeV}$, the Higgs boson tends to decay to a pair of bottom quarks with large branching ratios [21]. The final states are overwhelmed by the multi-jet background processes in hadron collisions. This makes it difficult to search for the low mass Higgs boson prouduction via gluon-gluon fusion. Since the Higgs boson prefers to couple to a heavy particle, it could be produced in association with a vector boson. The vector boson is relatively easy to be identified 
through its leptonic decay and distinguished from the large background in hadron collisions. This signature makes the assoicated productions of the Higgs boson with vector bosons the most promising channels to search for the Higgs boson with a mass less than $135 \mathrm{GeV}$ at the Tevatron.

This thesis is focused on searching for Higgs boson production associated with a $\mathrm{W}$ boson at the $\mathrm{D} \emptyset$ experiment of the Tevatron. The $\mathrm{W}$ boson is identified through its leptonic decay into a lepton and a neutrino, while the Higgs boson is reconstructed from a pair of bottom quarks. An overview on the Higgs mechanism in the Standard Model together with Higgs production and decay at hadron colliders is given in Chapter II. In Chapter III, I describe the Tevatron accelerator as well as the DØ detector in more details. Chapter IV includes the various algorithms of event reconstruction, correction and b-jet identification. The event samples and the event selection criteria are described in detail in Chapter V. In Chapter VI, multivariate techniques are implemented to further discriminate the Higgs signal from the background events. Finally, the conclusions and discussions on Higgs production at the Tevatron are given in Chapter VII. 


\section{CHAPTER II}

\section{The Standard Model Higgs Production and Decay in Hadron Collisions}

A brief overview of the Standard Model Higgs boson together with its predicted experimental signatures is given in this chapter. First of all, an introduction to the theoretical aspects of the Standard Model and Higgs boson is made through Section 2.1. The contraints on the Higgs boson mass from both theorectical calculations and experimental results are summarized in Section 2.2. The results on the Tevatron sensitivity to the Higgs boson production are also given in that section. In Section 2.3 the various mechanisms of the Higgs boson production and decay in hadron collisions are explained. The channel to search for the Higgs boson in this thesis is briefly discussed as well.

\subsection{The Standard Model, Higgs Mechanism and Higgs Boson}

\subsubsection{The Standard Model Theory}

The Standard Model theory in particle physics provides a quite elegant framework to describe the elementary particles and the interactions among these particles. It is a gauge theory built on the symmetry group $S U_{C}(3) \times S U_{L}(2) \times U_{Y}(1)$, which unifies the strong, weak and electromagnetic interactions in the nature.

A group of particles, called leptons and quarks, are known to make up the matter 


\begin{tabular}{|l|c|c|c|c|c|}
\hline & \multicolumn{3}{|c|}{ Generation } & Electric Charge & Spin \\
& 1 st & 2 nd & 3rd & & \\
\hline \multirow{2}{*}{ Leptons } & $\nu_{e}$ & $\nu_{\mu}$ & $\nu_{\tau}$ & 0 & $1 / 2$ \\
& $e$ & $\mu$ & $\tau$ & -1 & $1 / 2$ \\
\hline \multirow{2}{*}{ Quarks } & $\mathrm{u}$ & $\mathrm{c}$ & $\mathrm{t}$ & $2 / 3$ & $1 / 2$ \\
& $\mathrm{~d}$ & $\mathrm{~s}$ & $\mathrm{~b}$ & $-1 / 3$ & $1 / 2$ \\
\hline
\end{tabular}

Table 2.1: List of leptons and quarks in the Standard Model

\begin{tabular}{|l|c|c|c|c|c|}
\hline Gauge Bosons & Interactions & Fields & Symmetry Group & Charge & Spin \\
\hline$\gamma$ & Electromagnetic & $A_{\mu}$ & $U_{Y}(1)$ & 0 & 1 \\
\hline$Z, W^{ \pm}$ & Weak & $Z_{\mu}, W_{\mu}^{ \pm}$ & $S U_{L}(2)$ & $0, \pm 1$ & 1 \\
\hline Gluons & Strong & $g_{\mu}^{a}$ & $S U_{C}(3)$ & 0 & 1 \\
\hline
\end{tabular}

Table 2.2: List of gauge bosons in the Standard Model

in the world. One distinct feature is that the spins of these particles are $1 / 2$, for which they are called fermions. These matter particles are further organized into three generations, which are summarized in Tab. 2.1. Each particle in the table has its counterpart known as antiparticle with opposite electric charge. The particles and their corresponding antiparticles are depicted using fields in the theory. The electron, muon, tau and quarks have both left-hand and right-hand chiral components, while the neutrinos are considered to be only left-handed. The quarks are color triplets in the $S U_{C}(3)$ group while leptons are color singlets. The fields preserve the renormalizability of electroweak theory [22].

The interactions among the fermions are mediated by another group of particles, called gauge bosons, of which the spin is 1 . The gauge bosons and corresponding interactions are summarized in Tab. 2.2. The photon $\gamma, Z, W_{\mu}^{ \pm}$bosons and gluons mediate the electromagnetic, weak and strong forces respectively.

The Standard Model theory unifies the strong, weak and electromagnetic interactions through symmetry group $S U_{C}(3) \times S U_{L}(2) \times U_{Y}(1)$. The Lagrangian is generally used to describe the dynamics of the entire physical system. The Lagrangian density 
for the interactions in the Standard Model theory is given by

$$
\begin{aligned}
\mathcal{L}_{S M}= & -\frac{1}{4} G_{\mu \nu}^{a} G_{a}^{\mu \nu}-\frac{1}{4} W_{\mu \nu}^{a} W_{a}^{\mu \nu}-\frac{1}{4} B_{\mu \nu} B^{\mu \nu} \\
& +\bar{L}_{i} i D_{\mu} \gamma^{\mu} L_{i}+\bar{e}_{R_{i}} i D_{\mu} \gamma^{\mu} e_{R_{i}}+\bar{Q}_{i} i D_{\mu} \gamma^{\mu} Q_{i}+\bar{u}_{R_{i}} i D_{\mu} \gamma^{\mu} u_{R_{i}}+\bar{d}_{R_{i}} i D_{\mu} \gamma^{\mu} d_{R_{i}}
\end{aligned}
$$

where $\gamma^{\mu}$ are $4 \times 4 \gamma$ matrices, $D_{\mu}$ is the covariant derivative, $L_{i}$ and $Q_{i}(i=1,2,3)$ represent left-handed leptons and quarks in weak isodoubletes, $e_{R_{i}}, u_{R_{i}}$ and $d_{R_{i}}$ represent right-handed fermions in weak isosinglets. The field strengths of the different gauge fields in the $U_{Y}(1), S U_{L}(2)$ and $S U_{C}(3)$ groups are given by

$$
\begin{aligned}
& B_{\mu \nu}=\partial_{\mu} B_{\nu}-\partial_{\nu} B_{\mu} \\
& W_{\mu \nu}^{a}=\partial_{\mu} W_{\nu}^{a}-\partial_{\nu} W_{\mu}^{a}+g_{2} \epsilon^{a b c} W_{\mu}^{b} W_{\nu}^{c} \\
& G_{\mu \nu}^{a}=\partial_{\mu} G_{\nu}^{a}-\partial_{\nu} G_{\mu}^{a}+g_{s} f^{a b c} G_{\mu}^{b} G_{\nu}^{c}
\end{aligned}
$$

where $\epsilon^{a b c}$ and $f^{a b c}$ are the antisymmetric tensors, $g_{2}$ and $g_{s}$ are the coupling constants of $S U_{L}(2)$ and $S U_{C}(3)$ groups [1].

This Lagrangian is invariant under local gauge transformations for fermion and gauge fields. It successfully incorporates the three known fundamental forces into a unified theoretical framework. However, it is not yet able to explain the mass origins of the fermions and some gauge bosons, which have been experimentally observed to be massive. If the mass terms of fermions and gauge bosons, $-m_{f} \bar{\psi}_{f} \psi_{f}$ and $\frac{1}{2} M_{V}^{2} W_{\mu} W^{\mu}$, were explicitly added into the Lagrangian, the local gauge invariance of $S U_{L}(2) \times U_{Y}(1)$ would be broken. As an alternative solution, the Higgs mechanism of spontaneous symmetry breaking [23] [24] [25] [26] was proposed to give masses to particles. The mechanism is briefly reviewed in the following subsection. 


\subsubsection{Spontaneous Symmetry Breaking and the Higgs Mechanism}

The concept of spontaneous symmetry breaking is the basis on which the Higgs mechanism is introduced to the Standard Model theory. A physical system, which is initially symmetric regarding to certain symmetry group, may naturally go into a non-symmetric vacuum state and thus break the symmetry. The process can be illustrated using a scalar real field $\phi$, of which the Lagrangian is

$$
\mathcal{L}=\frac{1}{2} \partial_{\mu} \phi \partial^{\mu} \phi-V(\phi), \text { where } V(\phi)=\frac{1}{2} \mu^{2} \phi^{2}+\frac{1}{4} \lambda \phi^{4}
$$

The Lagrangian is invariant under the reflection symmetry $\phi \leftrightarrow-\phi$. Assuming $\mu^{2}<0$, the potential $V(\phi)$ has the minimum when $\phi= \pm \sqrt{-\frac{\mu^{2}}{\lambda}} \equiv v$, where $v$ is called the vacuum expectation value of $\phi$. In this case we have to make a perturbative expansion around the minimum $v$ by defining the field $\phi^{\prime}=\phi-v$. The Lagrangian in terms of the new field now contains a cubic term, for which the reflection symmetry is spontaneously broken. This study can be generalized to continuous symmetry as well. According to the Goldstone theorem [27], there would be massless scalar particles, called Goldstone bosons, when continuous symmetry is spontaneously broken.

The Higgs mechanism is then introduced to generate masses for gauge bosons $W^{ \pm}$and $Z$ in the Standard Model gauge theory. The simplest choice is to add the invariant term

$$
\mathcal{L}_{H}=\left(D^{\mu} \Phi\right)^{\dagger}\left(D^{\mu} \Phi\right)-\mu^{2} \Phi^{\dagger} \Phi-\lambda\left(\Phi^{\dagger} \Phi\right)^{2}
$$

where $\Phi$ is $S U_{L}(2)$ doublet of complex scalar fields $\Phi=\left(\begin{array}{c}\phi^{\dagger} \\ \phi^{0}\end{array}\right)=\frac{1}{\sqrt{2}}\left(\begin{array}{c}\phi_{1}+i \phi_{2} \\ \phi_{3}+i \phi_{4}\end{array}\right)$. In this case, $\Phi$ has the vacuum expectation value by preserving $U_{Y}(1)$ symmetry $<\Phi>_{0}=\frac{1}{\sqrt{2}}\left(\begin{array}{l}0 \\ v\end{array}\right)$ with $v=\sqrt{-\frac{\mu^{2}}{\lambda}}$. When making a gauge transformation on 
the field $\Phi$ to move to the unitary gauge, $\Phi$ can then be modified with $\Phi(x)=$

$\frac{1}{\sqrt{2}}\left(\begin{array}{c}0 \\ v+H(x)\end{array}\right)$. Rewriting the Lagrangian $\mathcal{L}_{\mathcal{H}}$ gives the new fields $W_{\mu}^{ \pm}$and $Z_{\mu}$, which are corresponding to $W^{ \pm}$and $Z$ bosons with masses $M_{W}=\frac{1}{2} v g_{2}$ and $M_{Z}=$ $\frac{1}{2} v \sqrt{g_{1}^{2}+g_{2}^{2}}$, where $g_{1}$ is the electromagnetic coupling constant.

The same exercise can be done for generating the fermion masses by introducing the $S U_{L}(2) \times U_{Y}(1)$ Yukawa Lagrangian with the same field $\Phi$

$$
\mathcal{L}_{\text {Yukawa }}=-\lambda_{e} \bar{L} \Phi e_{R}-\lambda_{d} \bar{Q} \Phi d_{R}-\lambda_{u} \bar{Q} \tilde{\Phi} u_{R}+\text { h.c. }
$$

where h.c. represents the Hermitian conjugate of the terms in the Lagrangian. The masses of the fermions are given by $m_{e}=\frac{\lambda_{e} v}{\sqrt{2}}, m_{u}=\frac{\lambda_{u} v}{\sqrt{2}}$ and $m_{d}=\frac{\lambda_{d} v}{\sqrt{2}}$.

In both cases, the same doublet complex field $\Phi$ is introduced to spontaneously break the $S U_{L}(2) \times U_{Y}(1)$ symmetry, while preserving the electromagnetic $U_{Y}(1)$ symmetry and $S U_{C}(3)$ color symmetry. As a result, the weak vector bosons $W^{ \pm}$, $Z$ and the fermions acquire their masses respectively, while the photon and gluons remain as massless bosons. Another important feature of the mechanism is the appearance of a massive scalar field, called the Higgs field. The Higgs field predicts a scalar particle, the Higgs boson, which is discussed in the next subsection.

\subsubsection{The Higgs Boson}

The Higgs boson is a massive scalar particle predicted by the Higgs mechanism, which is developed to give masses to the vector bosons. If the Higgs field related terms are picked from Eq. 2.4, a special Higgs Lagrangian is built as

$$
\mathcal{L}_{\text {Higgs }}=\frac{1}{2}\left(\partial^{\mu} H\right)^{2}-\lambda v^{2} H^{2}-\lambda v H^{3}-\frac{1}{4} \lambda H^{4}
$$

The Higgs boson mass is given by

$$
m_{H}=\sqrt{2 \lambda v^{2}}=\sqrt{-2 \mu^{2}}
$$


The vacuum expectation value $v$ is determined from the mass of the $W$ boson and the Fermi constant $G_{F}$

$$
v=\frac{2 m_{W}}{g_{2}}=\sqrt{\frac{\sqrt{2} g^{2}}{4 G_{F} g_{2}^{2}}} \simeq 246 G e V,
$$

where $G_{F}$ is measured from muon decay. However, the value of $\lambda$ is not known yet, which makes the mass of the Higgs boson a mystery in theory.

The Higgs boson interacts with gauge bosons, fermions and itself with different coupling strengths. The possible ways of the Higgs couplings are given by the interaction terms in the Lagrangians of Eq. 2.4 and 2.5. These interactions are summarized in terms of vertices with Feynman diagrams in Fig. 2.1. The diagrams show that

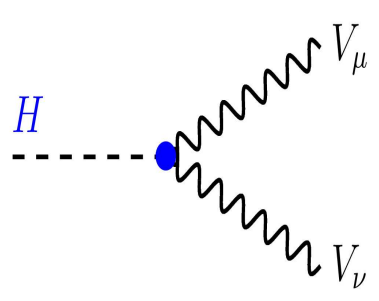

(a) $g_{H V V}=\frac{2 m_{V}^{2}}{v} \times\left(-i g_{\mu \nu}\right)$

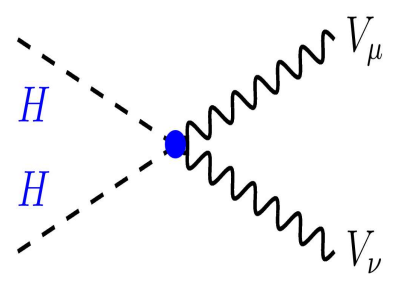

(b) $g_{H H V V}=\frac{2 m_{V}^{2}}{v^{2}} \times\left(-i g_{\mu \nu}\right)$

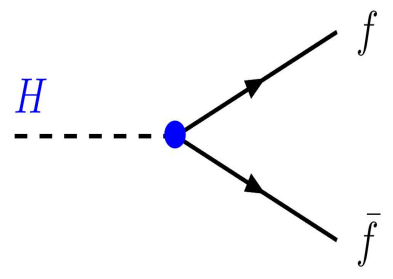

(c) $g_{H f f}=\frac{m_{f}}{v} \times(i)$

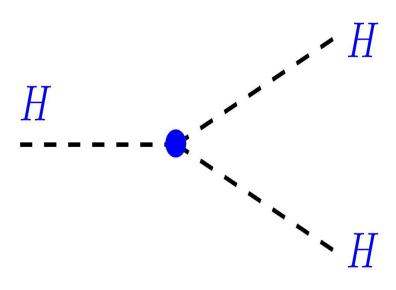

(d) $g_{H H H}=\frac{3 m_{H}^{2}}{v} \times(i)$

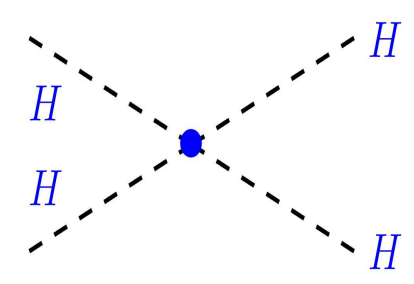

(e) $g_{H H H H}=\frac{3 m_{H}^{2}}{v^{2}} \times(i)$

Figure 2.1: The Higgs boson couplings to gauge bosons, fermions and itself in the Standard Model. The coupling parameters are respectively $g_{H V V}, g_{H H V V}, g_{H f f}, g_{H H H}$ and $g_{H H H H}$. [1]

the Higgs boson couples to particles in several ways in the Standard Model theory. The coupling strength of the Higgs boson to vector boson is proportional to the mass square of vector boson, while in the case of fermion it is proportional to the fermion 
mass. The self-coupling strengths of the Higgs boson are proportional to the mass square of Higgs boson itself. The coupling information is very useful in the following discussion on the potential production and decay of the Higgs boson in hadron collisions.

\subsection{A Review on the Searches for the Higgs Boson}

\subsubsection{Constraints on the Higgs Boson Mass}

In order to discover the Higgs boson, it would be useful to have some prior knowledge about its mass. The constraints on the Higgs boson mass so far have been achieved with different methods both theoretically and experimentally.

Most of the theoretical constraints are calculated based on the assumption that the Standard Model theory is valid up to certain energy scale, above which new physics beyond the Standard Model theory would come into being. The tree-level unitarity from scattering amplitudes of longitudinal vector bosons gives certain upper limits of $\mathcal{O}(700 \mathrm{GeV})$ [28]. Perturbativity studies from Higgs decay processes also suggest similar upper limits on the Higgs boson mass [29]. More stringent constraints are derived from the theory triviality and vacuum stability [2], which are based on high order calculations of Higgs boson quartic couplings with contributions from top quark and vector bosons. As is shown in Fig. 2.2, the constraints on Higgs boson mass varies with the new physics energy scale $\Lambda$. If the energy scale is at $\sim 1 \mathrm{TeV}$, the Higgs boson mass is allowed to be $50 \mathrm{GeV} \lesssim m_{H} \lesssim 800 \mathrm{GeV}$. If the Standard Model theory is valid up to the GUT scale $10^{16} \mathrm{GeV}$, the Higgs boson mass is going to be $130 \mathrm{GeV} \lesssim m_{H} \lesssim 180 \mathrm{GeV}$. In addition, when $\Lambda>1 \mathrm{TeV}$, the fine-tuning problem with the Higgs boson mass and its radiative corrections could further provide useful information about the Higgs boson mass [30].

There have been direct limits on the Higgs boson mass from high energy experi- 


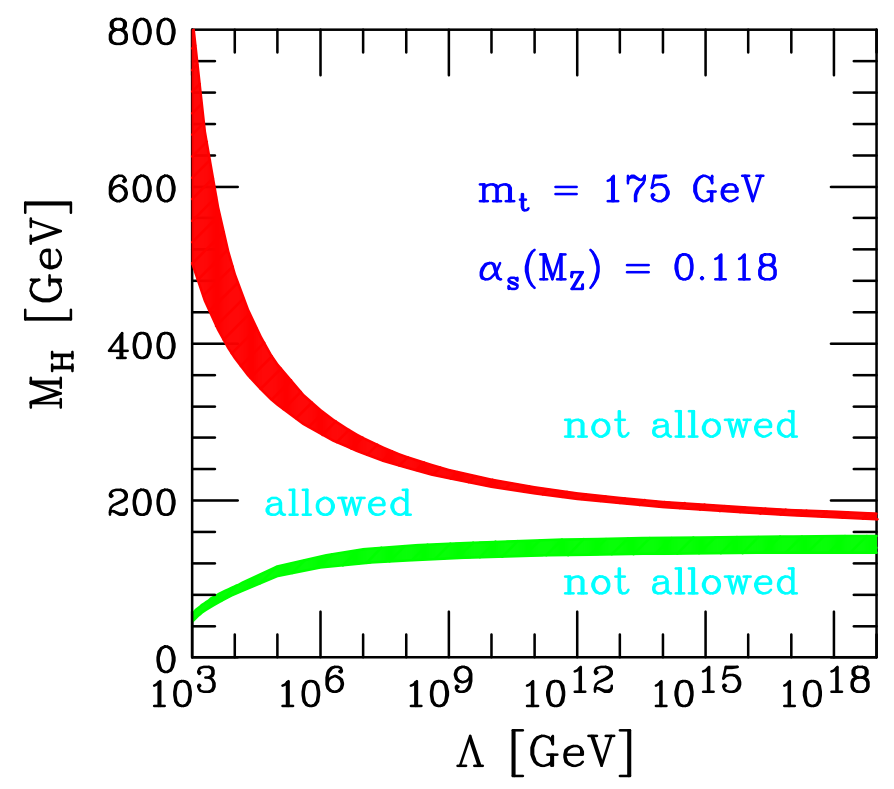

Figure 2.2: Constraints on the Higgs boson mass as a function of energy cutoff scale $\Lambda$ [2]. The upper bound is derived from triviality, while the lower bound is from the vacuum stability. The top quark mass and strong coupling constant in the calculation are $m_{t}=175 \pm 6 \mathrm{GeV}$ and $\alpha_{s}\left(M_{Z}\right)=0.118 \pm 0.002$ respectively.

ments. Direct searches for the Higgs boson have been performed by analyzing a total of $2461 \mathrm{pb}^{-1}$ data at the Large Electron-Positron Collider (LEP) experiment with $\sqrt{s}$ ranging from 189 to $209 \mathrm{GeV}$. No signal of Higgs boson was observed but a lower bound on Higgs boson mass is established to be $114.4 \mathrm{GeV}$ at $95 \%$ C.L. [31]. The most recent results at the Tevatron of $\sqrt{s}=1.96 \mathrm{TeV}$ exclude the existence of the Standard Model Higgs boson with $160 \mathrm{GeV}<m_{H}<170 \mathrm{GeV}$ at 95\% C.L. [20].

Rather strong constraints on the Higgs boson mass are indirectly obtained from high precision measurements of electroweak parameters. The idea is based on the fact that most of the electroweak parameters, such as the $W$ boson mass, are very sensitive to Higgs boson mass through high order loop corrections. A set of electroweak observables, combined from high- $Q^{2}$ electroweak precision measurements at LEP, SLC and the Tevatron, are fed into a global fit of $\Delta \chi^{2}=\chi^{2}-\chi_{\min }^{2}$ as a function 
of Higgs boson mass. As shown in Fig. 2.3, the preferred Higgs boson mass corresponds to the minimum of the fitted curve. The shaded band represents theoretical uncertainties from unknown higher order corrections. The recent results suggest a Higgs boson mass of $m_{H}=87_{-26}^{+35} \mathrm{GeV}$, where the uncertainties are derived only from $\Delta \chi^{2}=1$ corresponding to $68 \%$ C.L.. An upper limit of $186 \mathrm{GeV}$ is also derived from $\Delta \chi^{2}=2.7$ including both the experimental and theoretical uncertainties at $95 \%$ C.L.. These constraints on the Higgs boson mass provide useful guidance to further searches for the Standard Model Higgs boson.

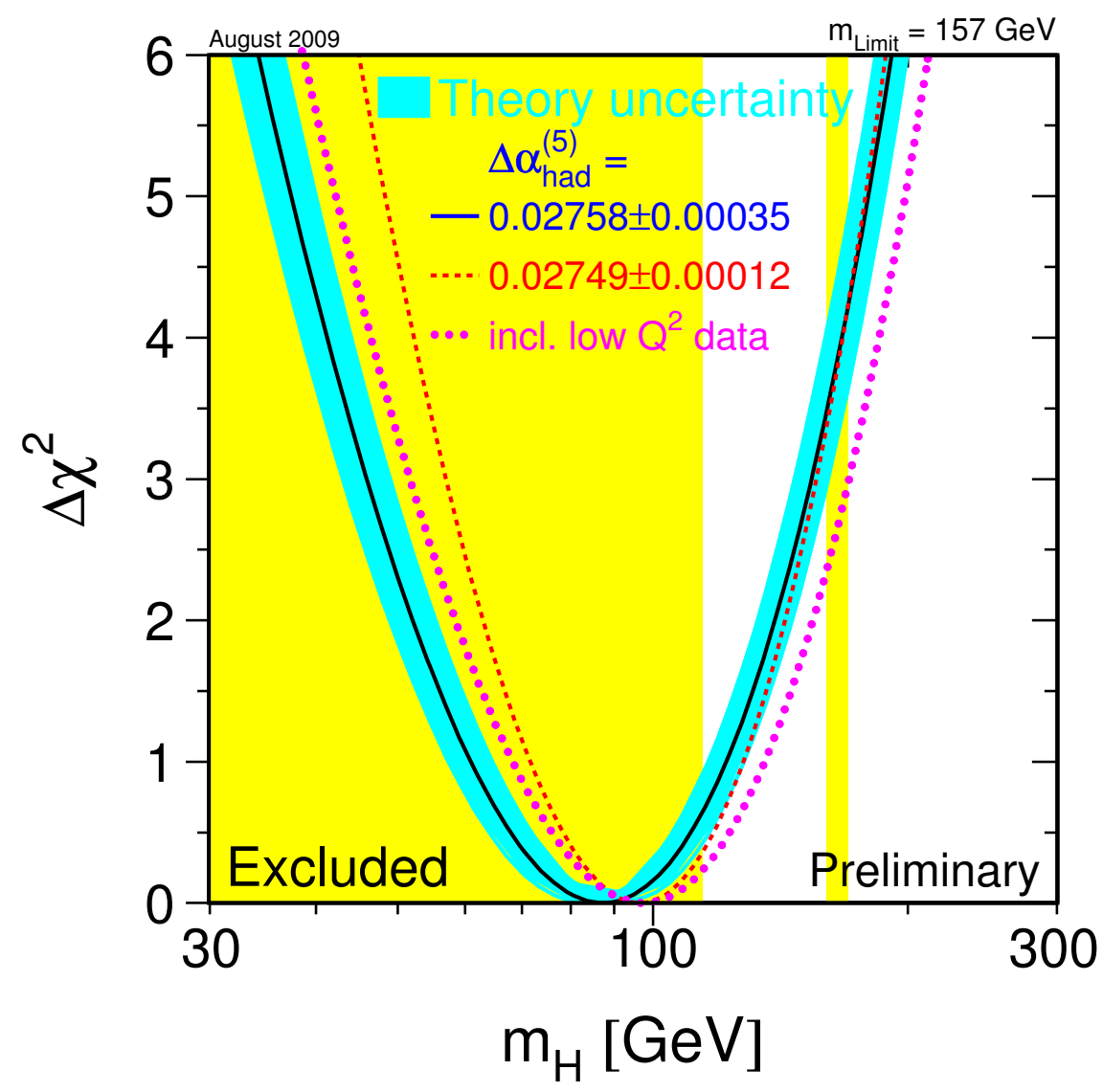

Figure 2.3: The $\Delta \chi^{2}=\chi^{2}-\chi_{\min }^{2}$ of the fit to electroweak precision data as a function of Higgs boson mass $m_{H}$. The solid line is the fitted value including all high- $Q^{2}$ data. The shaded band represents theoretical uncertainty from unknown higher order corrections. The vertical bands are the exclusion areas resulted from direct searches for Higgs boson at LEP and the Tevatron. Also shown in the plot are uses of different $\Delta \alpha_{\text {had }}$ values and inclusion of low- $Q^{2}$ data. [3] 


\subsubsection{Search for the Higgs Boson at the Tevatron}

The constraints on the Higgs boson mass prefer a light Higgs boson with mass range below $200 \mathrm{GeV}$, which gives the Tevatron great potential to observe this particle. At present the Tevatron is the only running accelerator in the world capable of direct Higgs boson search before the LHC turning into practical operation. The results of studies on the Higgs sensitivity at the Tevatron are summarized in Fig. 2.4 [4], while the thicker curves are the results of SHWG study in 1999 [32]. In the mass range $110-130 \mathrm{GeV}$, the Tevatron could be able to exclude the existence of the Higgs boson at $95 \%$ C.L. with $4 \mathrm{fb}^{-1}$ data, while a $3 \sigma$ evidence could be achieved with $8 \mathrm{fb}^{-1}$ data per experiment.

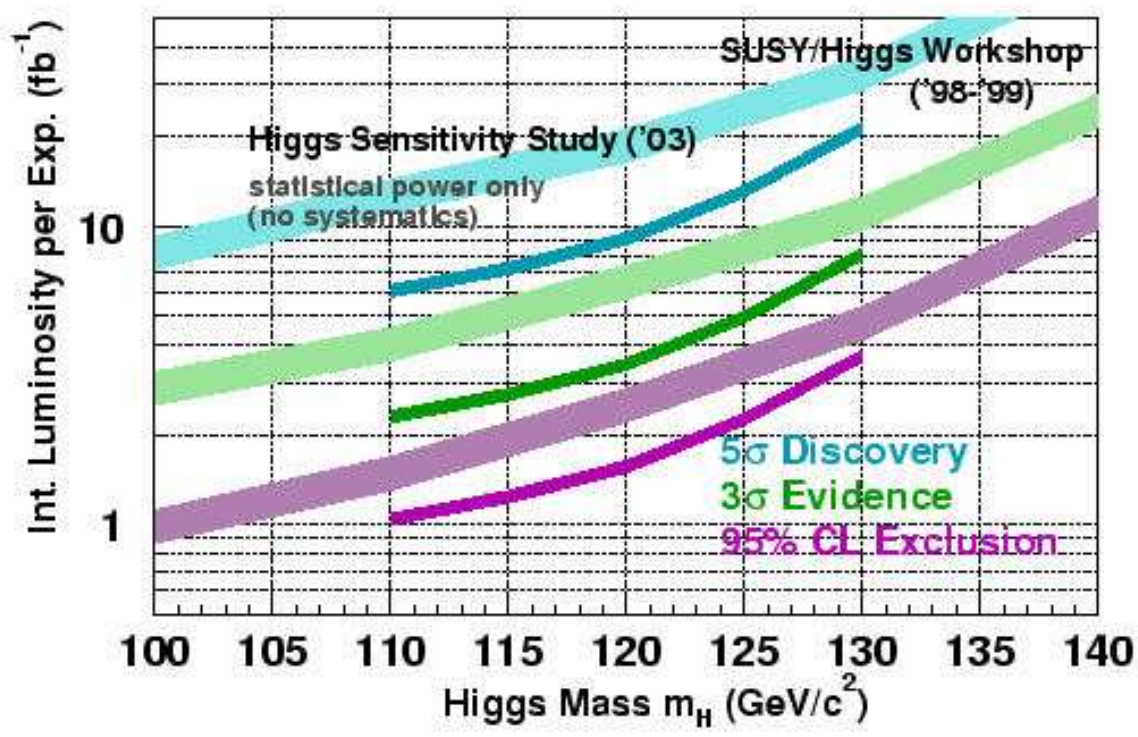

Figure 2.4: The sensitivity of the Higgs boson at the Tevatron. The corresponding integrated luminosities as a function of Higgs boson mass are expected to have exclusion at $95 \%$ C.L., $3 \sigma$ evidence and $5 \sigma$ discovery. The narrow curves are the updated studies in 2003, while the thicker curves are the results of SHWG study in 1999. [4] 


\subsection{The Higgs Boson Production and Decay at Hadron Colliders}

\subsubsection{Production of the Standard Model Higgs Boson}

The Higgs boson production in hadron collisions is dominated by those processes in which the Higgs bosons are coupling to heavy particles. This is based on the fact that the coupling strengths of the Higgs bosons to vector bosons and fermions increase with the masses of those particles. Particularly, in the modern proton colliders with energies $\sim \mathrm{TeV}$ scale, the Higgs boson tends to couple with vector bosons $W^{ \pm}, Z$ and heavy fermions such as top and bottom quarks. The major production mechanisms for a Higgs boson at the present hadron colliders are gluon-gluon fusion, vector boson fusion, the associated production with $W^{ \pm} / Z$ bosons and the associated production with heavy quarks [1].

The gluon-gluon fusion mechanism contributes to the largest part of Higgs production in hadron-hadron collisions. It is a process mediated by the triangular loops of heavy quarks. As is seen in Fig. 2.5(a), gluons from the colliding beams couple to a heavy quark loop which emits a Higgs boson. The loop is most likely made up of top quarks while it could be contributed by bottom quarks with a smaller chance. The Higgs boson coupling linearly grows with the quark mass whereas the heavy loop mass decreases the form factor of $\mathrm{Hgg}$. The Higgs boson production cross section via this process is significantly larger than those of other processes.

Vector boson fusion refers to the process whereby the quarks in the colliding beams emit virtual vector bosons, which in turn annihilate and produce a Higgs boson. As is shown in 2.5(b), $V^{*} V^{*}$ could be a pair of $W$ bosons or $Z$ bosons. This process is largely contributed by longitudinal gauge bosons and hence gives higher production rates with higher colliding energies. The $W W$ fusion channel has a larger production cross section than the $Z Z$ channel because $W$ bosons have larger 
couplings to fermions than $Z$ bosons.

The Higgs boson can also be produced in association with a vector boson in hadron collisions. The process is illustrated in Fig. 2.5(c). It can be simply viewed as a Drell-Yan process. A quark and antiquark annihilate to create a virtual vector boson, which is then realized as a pair of Higgs boson and vector boson through its bremsstrahlung process. The center-of-mass energy must be well above the threshold of the vector boson. The production cross section of $H W$ is roughly two times of $H Z$.

There are several processes in which the Higgs boson is associated with heavy quarks at hadron colliders. The common feature is that the heavy quark emits a Higgs boson. An example with internal quark line is shown in Fig. 2.5(d). At tree level, the incoming gluons in the colliding beams annihilate into heavy quarks from which a Higgs boson is produced. When the energy is higher and the gluon becomes important, it can happen through gluon fusion with the Higgs boson emitted from both the internal and external quark lines. The heavy quarks here mainly refer to top quarks and bottom quarks. The cross sections of $b \bar{b} H$ are comparable to and even larger than those of $t \bar{t} H$ at a certain level of colliding energy, thanks to the available phase space. The production of Higgs boson with single top quark is considerable regarding to the association production with top pair.

The Higgs boson production cross sections via these processes at hadron colliders have been calculated up to NNLO level [33]. Fig. 2.6 is the cross section as a function of Higgs boson mass at the Tevatron collider of $\sqrt{s}=1.96 \mathrm{TeV}$. The Higgs boson mass of the interest ranges from $100 \mathrm{GeV}$ to $200 \mathrm{GeV}$. As indicated in the plot, the dominant production is gluon fusion $g g \rightarrow H$, of which the cross section ranges from above $1 \mathrm{pb}$ to around $0.2 \mathrm{pb}$. The overall theoretical uncertainty is estimated to be 


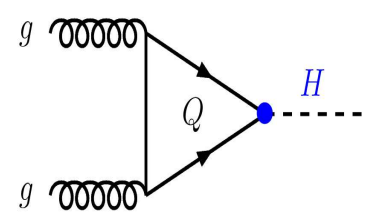

(a) $g g \longrightarrow H$

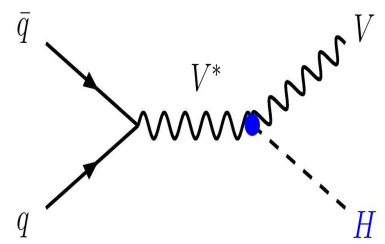

(c) $q \bar{q} \longrightarrow V+H$

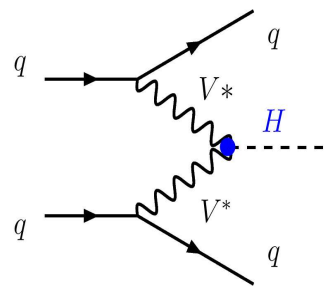

(b) $q q \longrightarrow V^{*} V^{*} \longrightarrow H$

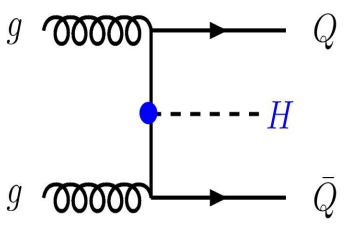

(d) $g g, q \bar{q} \longrightarrow Q \bar{Q}+H$

Figure 2.5: The major production processes of the Standard Model Higgs boson at hadron colliders. [1] (a) gluon-gluon fusion. (b) vector boson fusion. (c) associated production with $W^{ \pm}$ and $Z$ bosons. (d) associated production with heavy quarks.

around $10 \%$. The most relevant production of a low mass Higgs boson is associated with a vector boson $q q \rightarrow W H$ and $q q \rightarrow Z H$, as explained in the next sections. The cross section is calculated at NNLO in the QCD expansion and NLO in the electroweak correction. In the Higgs boson mass range from $100 \mathrm{GeV}$ to $200 \mathrm{GeV}$, the cross section decreases with Higgs boson mass from $0.3 \mathrm{pb}$ to $0.01 \mathrm{pb}$. The overall theoretical uncertainty of this process is less than $5 \%$.

\subsubsection{Decay of the Standard Model Higgs Boson}

In the Standard Model theory, once the mass of Higgs boson is specified, the possible decay modes and the corresponding decay widths of Higgs boson can be predicted. Generally the Higgs boson could decay to quarks, leptons, real or virtual vector bosons and even photons or gluons with a loop involved [1], as can be classified in the following: (1) $H \rightarrow f \bar{f}$. Higgs boson decays to a pair of fermions such as $b \bar{b}$, 
SM Higgs production

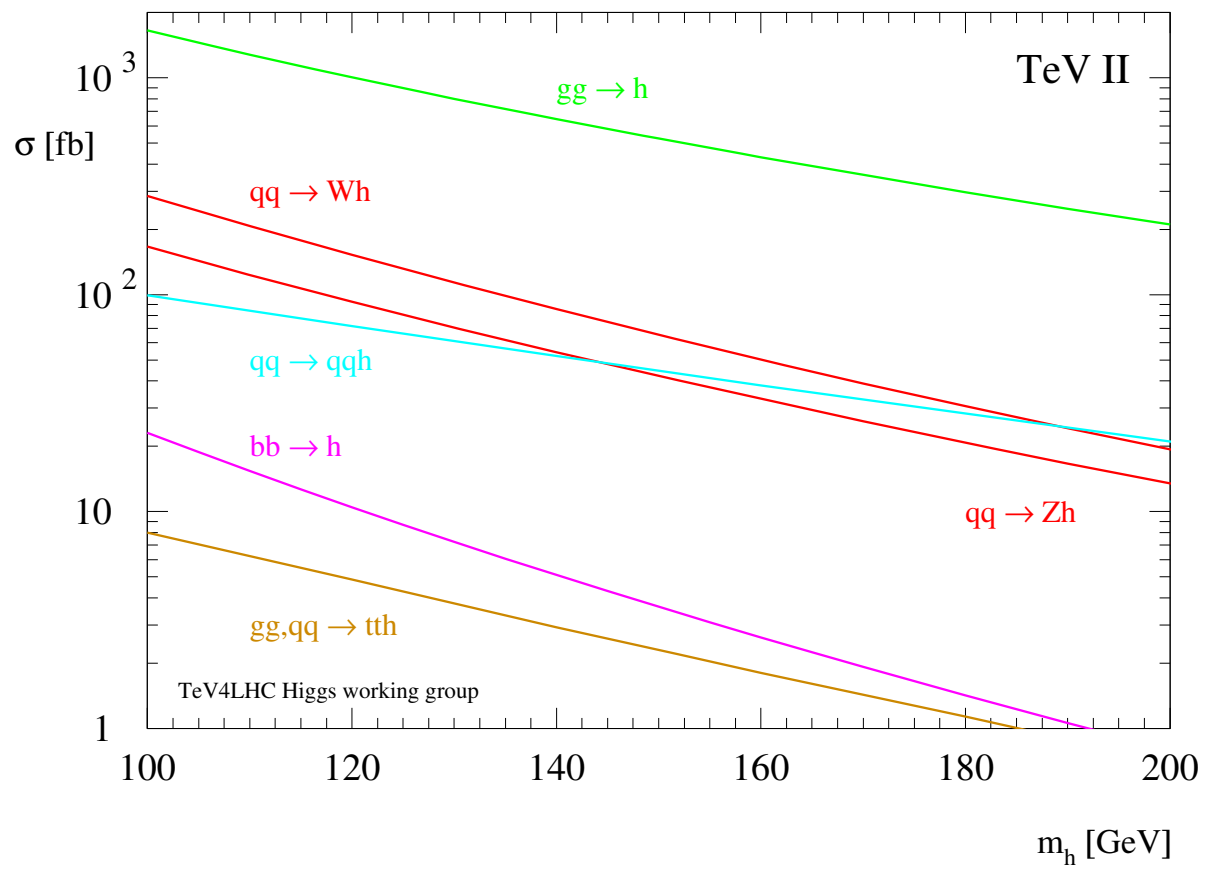

Figure 2.6: The cross sections of the Standard Model Higgs boson production as a function of the Higgs boson mass. Results are for the main production mechanisms at the Tevatron of $\sqrt{s}=1.96 \mathrm{TeV}$. The calculation is valid at NNLO in the QCD expansion for $g g \rightarrow H$, $q \bar{q} \rightarrow V H$ and $b \bar{b} \rightarrow H$, while it's accurate at NLO for $q q \rightarrow q q H$ and $g g, q q \rightarrow t \bar{t} H$.

$\tau^{+} \tau^{-}, c \bar{c}, s \bar{s}, \mu^{+} \mu^{-}$and $t \bar{t}$. (2) Higgs boson decays to massive vector bosons. $H \rightarrow V V$ when it is above kinematic thresholds of $W W$ or $Z Z$. $H \rightarrow V V^{*} \rightarrow V f \bar{f}$ with one off-shell boson, while $H \rightarrow V^{*} V^{*} \rightarrow f_{1} \bar{f}_{2} f_{3} \bar{f}_{4}$ with both of the bosons off-shell. (3) Although the Higgs boson has no direct couplings to gluons or photons at tree level, it could decay to these particles via loops. $H \rightarrow g g$ is mediated by heavy quark loops. $H \rightarrow \gamma \gamma$ and $H \rightarrow \gamma Z$ are enabled with $W$ boson or charged fermion loops.

As discussed in 2.1.3, the strengths of Higgs boson couplings to fermions and gauge bosons increase with the masses of these particles. Hence the Higgs boson preferentially decays to heavy particles as allowed by phase space. The decay widths and branching ratios are strongly dependent on the mass of the Higgs boson.

The total decay width of the Standard Model Higgs boson can be calculated using program HDECAY [5]. As is shown in Fig. 2.7, when the mass of Higgs boson is 


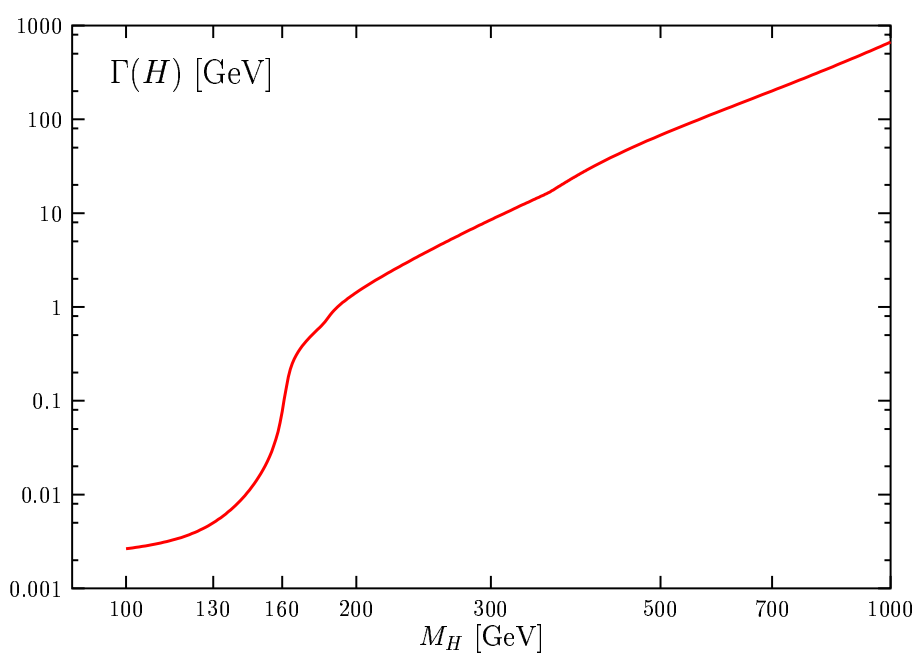

Figure 2.7: The decay width $\Gamma(H)$ as a function of Higgs boson mass in the Standard Model [1]. The data for Standard Model Higgs boson decay is obtained using the program HDECAY $[5]$.

smaller than $\sim 130 \mathrm{GeV}$, it has a very narrow width $\Gamma(H)<10 \mathrm{MeV}$. But the width quickly becomes wider up to $\sim 1 \mathrm{GeV}$ as the Higgs boson mass is increased to the threshold of $Z Z$. With larger Higgs boson masses, the decay width is comparable to the Higgs boson mass itself because of the longitudinal contributions from vector bosons.

The branching ratios of Higgs boson decays are summarized in Fig. 2.8. When the mass of Higgs boson is below $135 \mathrm{GeV}$, the process $H \rightarrow b \bar{b}$ is the dominant decay mode with branching ratio up to $80 \%$. The contributions from $\tau^{+} \tau^{-}, g g$ and $c \bar{c}$ are at several percent level. The branching ratios of $W W$ and $Z Z$ increase dramatically with the Higgs boson mass. When the Higgs boson mass is larger than $135 \mathrm{GeV}$, $W W^{*}$ becomes the main branch of Higgs boson decay. Note that the drop of $Z Z^{*}$ branching ratio when Higgs boson mass is between $160 \mathrm{GeV}$ and $180 \mathrm{GeV}$, is due to the fact that one $\mathrm{Z}$ boson remains off-shell while the threshold of $W W$ has been 


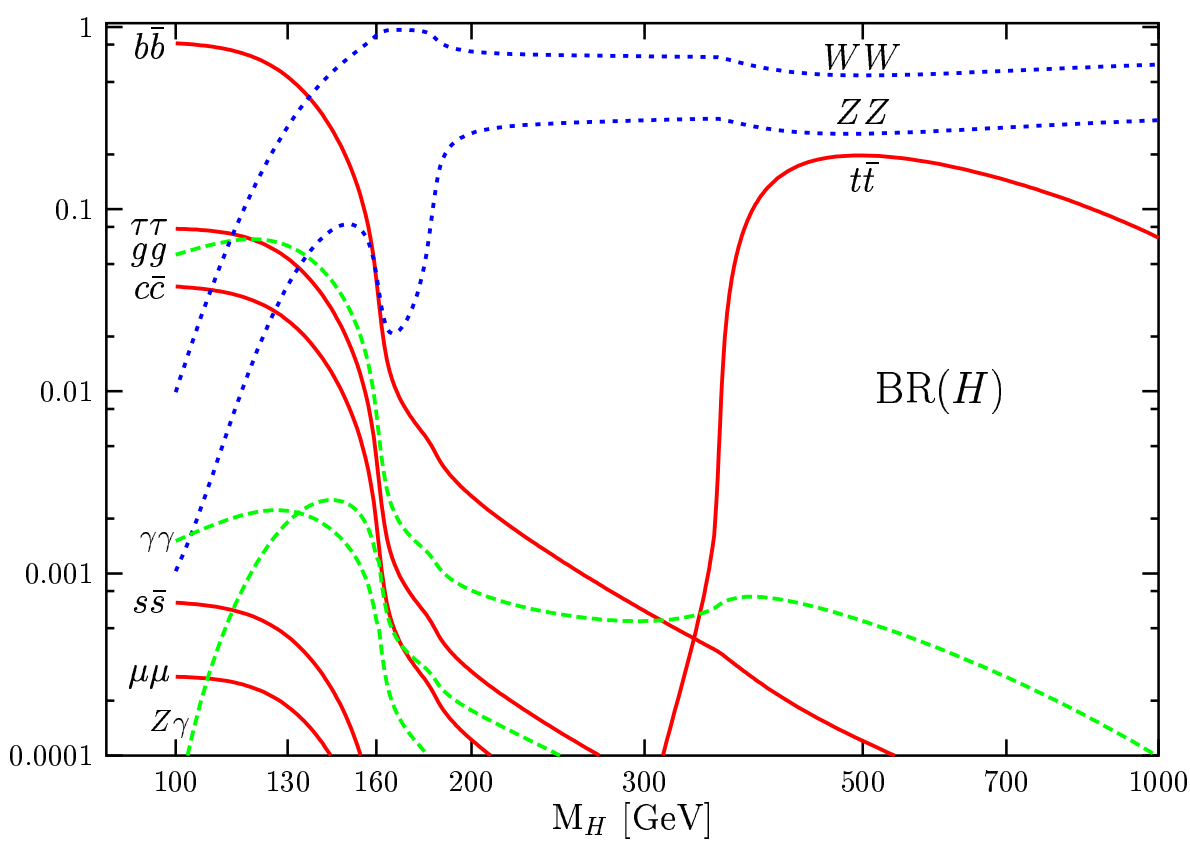

Figure 2.8: The branching ratios $B R(H)$ as a function of Higgs boson mass in the Standard Model [1], as calculated using the program HDECAY [5].

reached. When the Higgs boson mass is larger than $180 \mathrm{GeV}, W W$ and $Z Z$ dominate the Higgs boson decay. There is around a 10\% contribution from top quark pairs when the mass reaches the kinematic threshold of $t \bar{t}$. These signatures together with the Higgs boson production features provide guidance to Higgs boson searches, which are briefly discussed in the next section.

\subsubsection{Search for $p \bar{p} \rightarrow W^{ \pm} H \rightarrow l \nu b \bar{b}$ at the Tevatron}

As discussed in Sec.2.3.1 and 2.3.2, the gluon fusion process is the dominant production mechanism of Higgs boson at the Tevatron. If the mass of Higgs boson is larger than $135 \mathrm{GeV}$, it will mainly decay to a pair of vector bosons, which can be reconstructed to look for a Higgs boson. However, when it comes to the lower mass region $m_{H}<135 \mathrm{GeV}$, the Higgs boson dominantly decays to $b \bar{b}$. The process is overwhelmed by huge multijet processes in hadron-hadron collisions and thus difficult to identify the existence of a Higgs boson. The associated production of the Higgs 
boson with vector bosons naturally becomes the ideal channel for Higgs searches. As is shown in Fig. 2.9 [6], the production cross sections times branching ratios of $V H$ processes are considerable in the lower mass region of the interest.

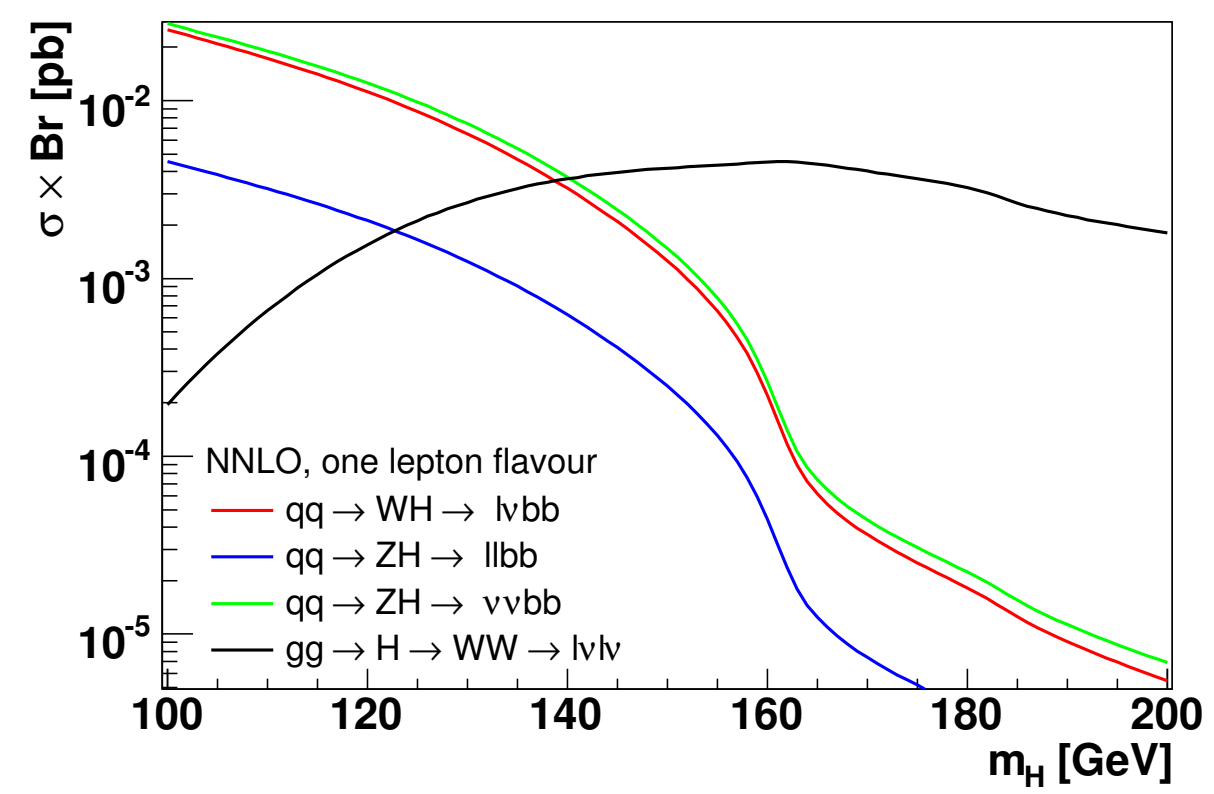

Figure 2.9: The Higgs production cross section times the branching ratios of main decay modes at the Tevatron as a function of Higgs boson mass. The NNLO cross sections of Higgs boson production are used in the calculation. The branching ratios of Higgs boson decay and $W / Z$ boson decay with single lepton flavor have been taken into account. [6]

The analysis presented in this thesis is based on the process $p \bar{p} \rightarrow W^{ \pm} H \rightarrow$ $l \nu b \bar{b}$ with the Higgs boson mass range from $100 \mathrm{GeV}$ to $150 \mathrm{GeV}$. In this case, the Higgs boson is produced in association with a $W$ boson in proton-antiproton collisions. The Higgs boson decays to a pair of b quarks, while the $\mathrm{W}$ boson could leptonically decay to a lepton and a neutrino. Although the signal has a relatively clean signature, the expected large background makes this analysis challenging. The main background processes come from $W$ plus multiple jets, of which particularly $W$ plus two b jets is irreducible. Some other physical processes, such as top quark and diboson productions, contribute signigicantly to background processes. The strategies and techniques used in this analysis to discriminate the signal from the 
background are discussed in the following chapters. 


\section{CHAPTER III}

\section{The Tevatron and the DØ Detector}

The Tevatron accelerator has been the energy frontier of hadron collisions for experimental particle physics research since it was established in 1983. It is now the only running particle accelerator capable of directly searching for the Higgs boson until the LHC begins running at CERN. In section 3.1, an overview of the chain of the accelerators at the Tevatron is presented. Since the data used in this analysis were collected at the $\mathrm{D} \varnothing$ detector, an introduction to the $\mathrm{D} \varnothing$ detector system is given in section 3.2 .

\subsection{The Tevatron Accelerator}

\subsubsection{An Overview of the Accelerators at the Tevatron}

The Tevatron accelerator at Fermilab delivers proton-antiproton collisions at centerof-mass energy of $1.96 \mathrm{TeV}$, which is the highest energy so far achieved by particle accelerators. A broad range of particle physics research has been carried out at the Tevatron, including the discovery of top quark. The search for the Higgs boson is one of the major physics goals at its two colliding detectors, CDF and D $\varnothing$.

The protons and antiprotons are created and accelerated through a complex chain of accelerators, of which an overview is presented in Fig. 3.1 [7]. The proton sources are produced in the Booster by stripping the electrons off negative hydrogen ions 
from the Linac. The antiprotons are accumulated from a target station where a target is bombarded by proton beams from the Main Injector. Both the beams are then accelerated and finally injected to the Tevatron ring, in which protons and antiprotons are accelerated up to $980 \mathrm{GeV}$ respectively and collided at the CDF and D $\varnothing$ detectors. The protons from the Booster and Main Injector are also delivered to fixed target experiments such as MINOS and MiniBooNE. Below are brief summaries of the accelerators in the chain. The technical details are available in reference [10].

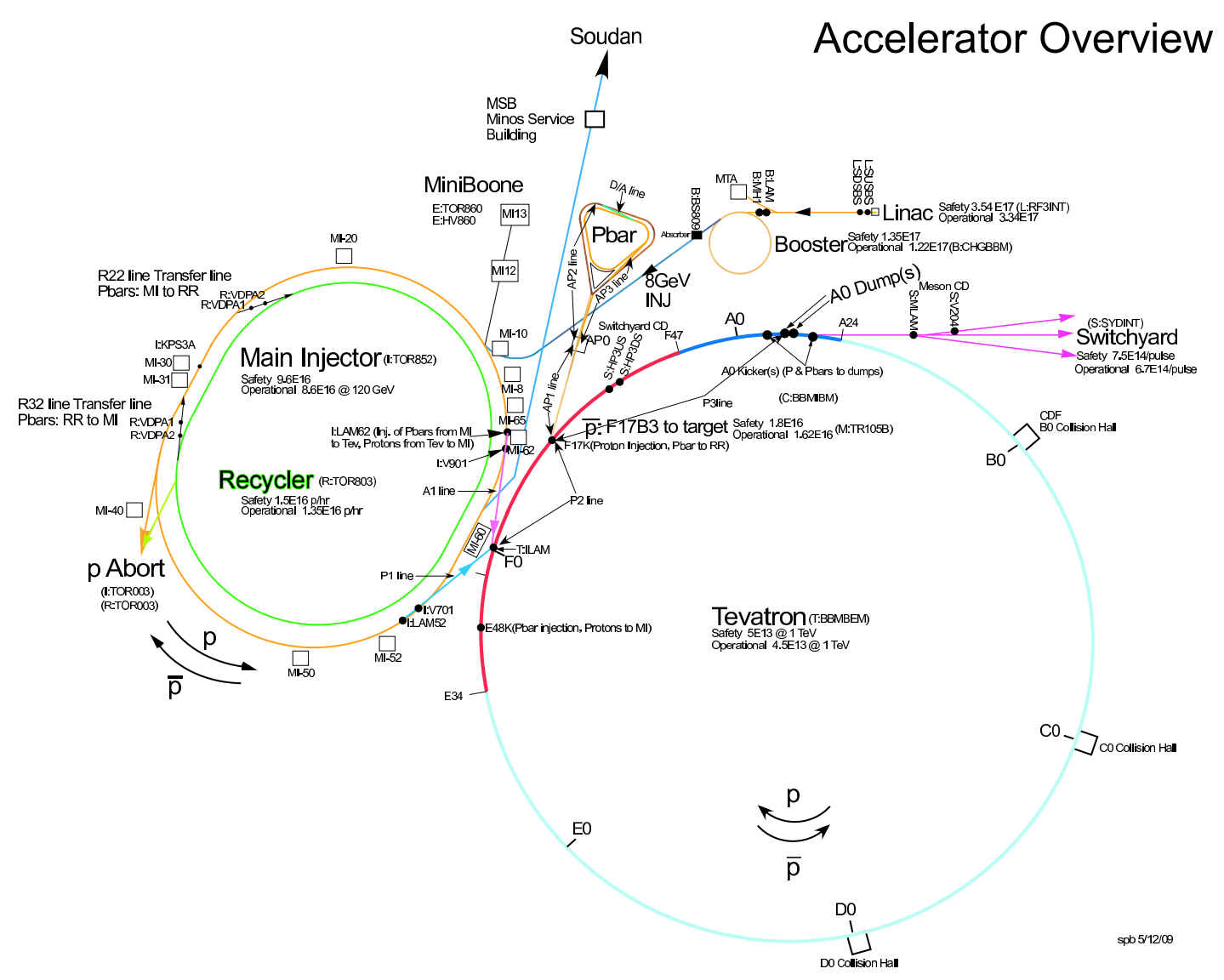

Figure 3.1: An overview of the accelerators at Fermilab. [7]

The particle beams originate from negative hydrogen ions accelerated by the Cockcroft-Walton accelerator. The hydrogen ions $H^{-}$are produced using magnetron surface plasma source [8], as described in Fig. 3.2. Generated from the cathode 
coated with cesium vapor, the $H^{-}$ions are extracted through the anode aperture and then accelerated to $18 \mathrm{KeV}$ through the extractor plate. These ion sources enter the Cockcroft-Walton accelerator, which utilizes static electric fields to accelerate charged particles. As shown in Fig. 3.3, the high voltage is generated by charging capacitors in parallel and discharging them in series with an $\mathrm{AC}$ power source. The Fermilab Cockcroft-Walton accelerator has five stages in total which result $750 \mathrm{KV}$ high voltage output. Thus the $H^{-}$ions are accelerated up to $750 \mathrm{KeV}$ through the Cockcroft-Walton accelerator and passed to the Linac accelerator.

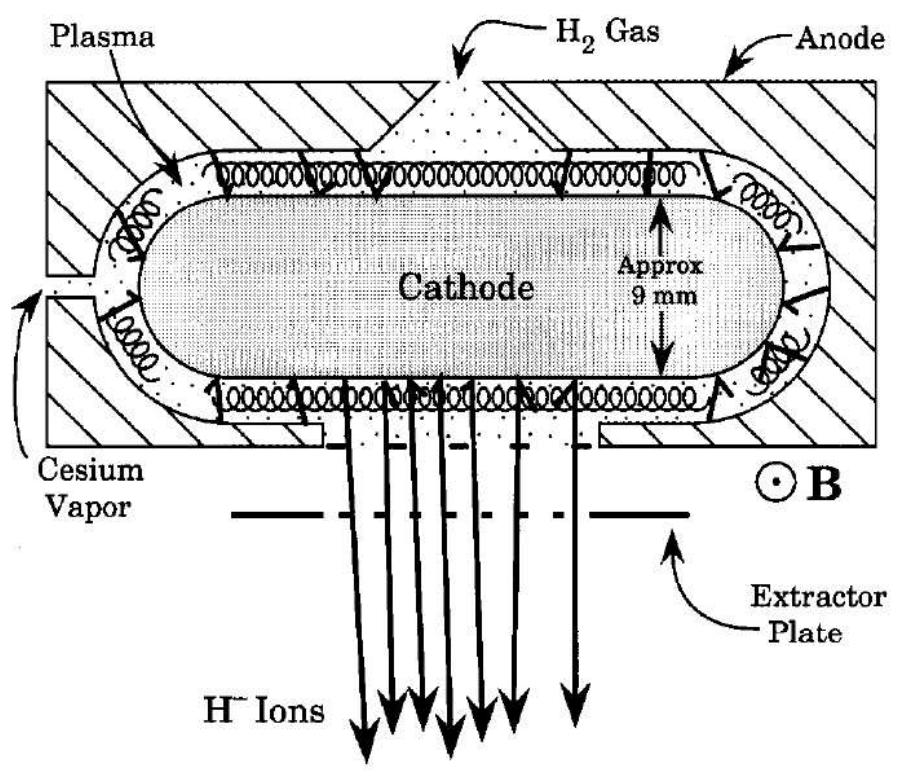

Figure 3.2: The basic configuration of the magnetron surface plasma source. [8]

The next level accelerator, the Linac, takes the $H^{-}$ions of $750 \mathrm{KeV}$ and accelerates them to an energy of $400 \mathrm{MeV}$. The Linac accelerator consists of two sections, the drift tube Linac and the side coupled cavity Linac. The drift tube Linac is a line of drift tubes radially centered in five cylindrical RF tanks, as is shown in Fig. 3.4. The $\mathrm{RF}$ tanks resonate at $201.24 \mathrm{MHz}$ and in total are able to accelerate the ion beams to an energy of $116 \mathrm{MeV}$. The side coupled cavity Linac has seven RF tanks similar to those in the drift tube Linac, but its drift tubes are designed to be more efficient. 


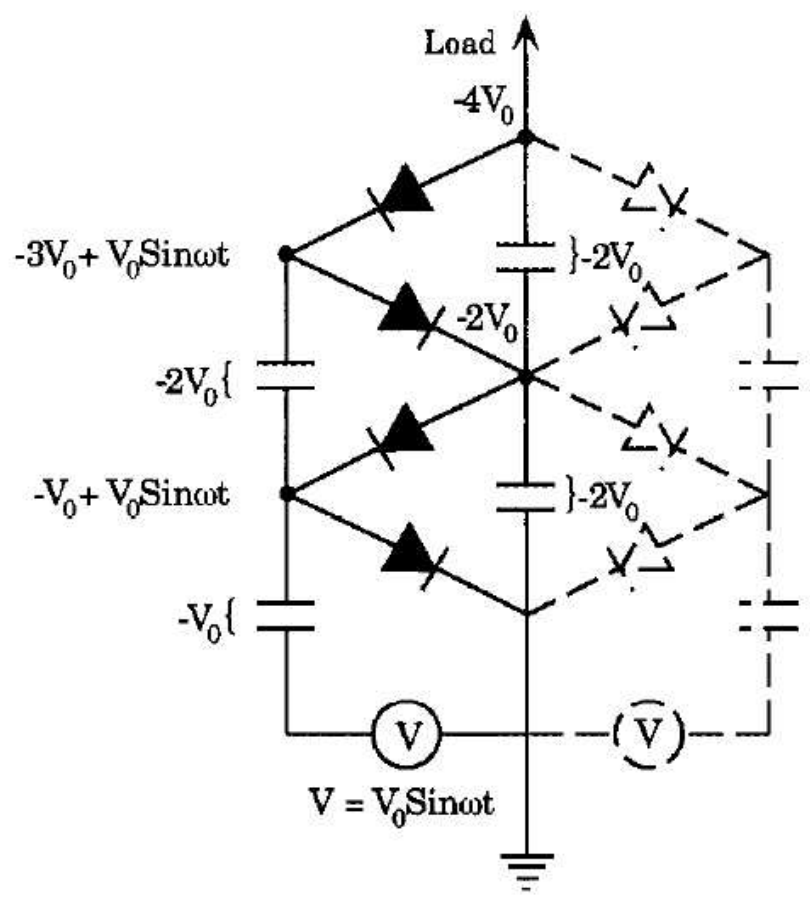

Figure 3.3: Schematic diagram of a simple two-stage diode voltage multiplier. Each stage of the chain yields $2 V_{0}$ output. The dotted lines represent special addition in the five-stage Fermilab Cockcroft-Walton accelerator. The purpose of this addition is to reduce the ripple while yielding the same output. [9]

Its RF tanks resonate at $805 \mathrm{MHz}$ and thus accelerate the beams every fourth $\mathrm{RF}$ cycle. Note that the beams in the Linac are present in the forms of bunches and are focused or defocused using quadrupole magnets. The ion beams are accelerated to an energy of $400 \mathrm{MeV}$ at this stage and transferred to the Booster.

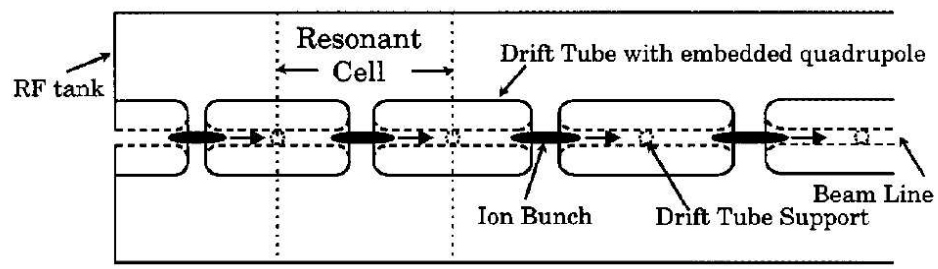

Figure 3.4: A simplified top view of Alvarez drift tube linac. A RF tank contains $n+1$ resonant cells. Each cell is filled with a bunch of particles. The particles are alternatively focused and defocused using quadrupole magnets embedded within the drift tubes. The bunches of particles are always accelerated in the gap between drift tubes while they are shielded in the drift tubes from the field of RF tank. [9]

The Booster is a circular synchrotron with a radius of $75 \mathrm{~m}$, where the $H^{-}$ion 
beams are stripped of their electrons and then accelerated to $8 \mathrm{GeV}$. The operation of the Booster can be divided into three stages. The first stage is the accumulation of protons. The incoming $H^{-}$ion beams pass through a $\mathrm{RF}$ debuncher which reduces the momentum spread of the bunches. The beams are then deflected and merged into a single beam with the already accumulated $H^{+}$beam using opposite dipole magnets. The new beam is then passed through a thin carbon foil to strip the electrons away from the $H^{-}$ions. Typically there will be $3 \times 10^{12}$ protons accumulated in six turns. The second stage is the acceleration of the protons. The protons are accelerated to 8 $\mathrm{GeV}$ in a closed orbit with increasing resonant frequencies of $\mathrm{RF}$ stations. The beam is kept in its orbit using alternating gradient magnets which focus and defocus a beam using combined function dipole-quadrupole magnets. The Booster can accelerate beam at a frequency of $15 \mathrm{~Hz}$. The last stage is the dump of protons. Once the proton beam is accelerated to $8 \mathrm{GeV}$, it is then directed to the transfer line to the Main Injector.

The Main Injector is a larger synchrotron with a radius of around $525 \mathrm{~m}$. It performs multiple tasks by accelerating both protons and antiprotons. When the 8 $\mathrm{GeV}$ proton beams are transferred from the Booster, the Main Injector can accelerate the proton beams to either $120 \mathrm{GeV}$ or $150 \mathrm{GeV}$. The $120 \mathrm{GeV}$ proton beams are delivered to fixed target experiments and antiproton source target, while $150 \mathrm{GeV}$ proton beams are injected to the Tevatron ring. The Main Injector also accepts the $8 \mathrm{GeV}$ antiproton beams from the Recycler or the Accumulator and accelerates them to $150 \mathrm{GeV}$ to be delivered to the Tevatron ring. Note that the Main Injector coalesces several Booster proton bunches into a superbunch before the beams are injected to the Tevatron.

The antiprotons are produced and stored with a series of apparatus including 
the fixed target, the Debuncher, the Accumulator and the Recycler. The $120 \mathrm{GeV}$ proton beam from the Main Injector is directed and focused to strike the nickel alloy target, from which all sorts of secondary particles are produced. A lithium lens is used to collect the $8 \mathrm{GeV}$ antiprotons from the spray, as shown in Fig. 3.5. A dipole magnet is added to select the momentum of the particles. The efficiency of antiproton production is very low with typically 15 antiprotons produced for every one million protons striking the target.

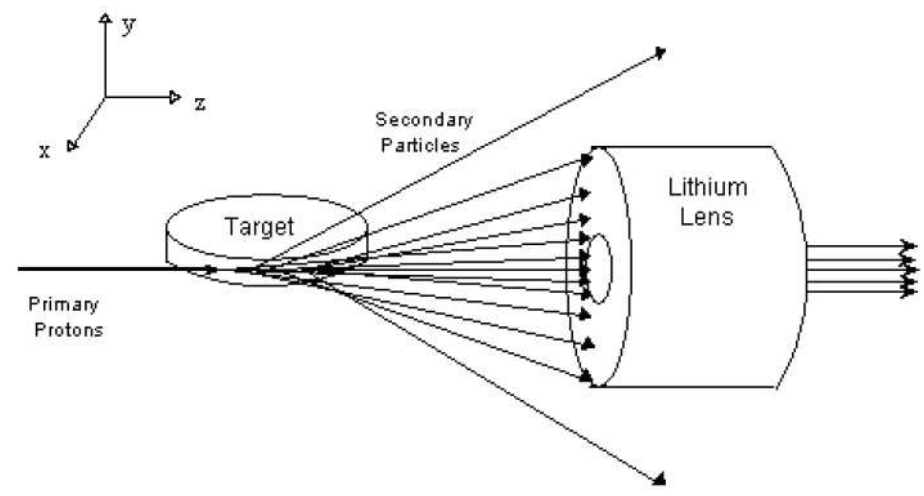

Figure 3.5: The lithium lens used to collect antiprotons from the secondary particles originated from the target. [10]

These extracted antiprotons are then diverted to the Debuncher, which is a triangular synchrotron used to reduce the high momentum spread of the antiprotons off the target. The process is realized through a method called stochastic cooling. A signal from circulating antiprotons on one side of the ring is picked indicating its deviation from the ideal orbit, while the resulted correction is applied to the antiproton beams on the other side of the ring to push it backward the ideal orbit. The same numbers of protons are compressed to smaller space size and energy spread through this method. The Accumulator is the second synchrotron housed in the same tunnel as the Debuncher. It is used as the storage ring of the antiprotons and also coincides the bunch structure with the Main Injector. Once the accumulation is completed, 
which usually takes more than twelve hours, the antiproton beams are directed to the Main Injector. The Recycler is housed right below the Main Injector ring and designed as a storage ring of antiprotons to allow higher stacking efficiency of the Accumulator. It uses both stochastic cooling and electron cooling systems. Electron cooling is based on the momentum transfer between the cool electron beams and antiproton beams. It is effective especially in high intensities. The accumulated antiproton beams are transfered to the Main Injector and then the Tevatron ring.

\subsubsection{The Tevatron, Cross Section and Luminosity}

The Tevatron ring is the main synchrotron accelerator where protons and antiprotons are accelerated to $980 \mathrm{GeV}$ respectively and collide at its two detectors. It has a circumference of approximately 4 miles. Its magnetic fields are up to $\sim 4 T$ provided by superconducting magnets which are cryogenically cooled by liquid helium to $\sim 4 K$.

The proton beams consisting of 36 bunches from the Main Injector are first injected to the Tevatron at $150 \mathrm{GeV}$ one bunch at a time. The antiproton beams with the same number of bunches and energy follow the same orbital paths but travel in the opposite direction. One bunch is separated from another typically by $396 \mathrm{~ns}$ orbiting in a series of FODO cells and correction magnets. The protons and antiproton are accelerated from $150 \mathrm{GeV}$ to $980 \mathrm{GeV}$. The two beams are then squeezed and collimated to collide by crossing each other in the center of the CDF and Dø detectors.

The colliding performance of the two beams is often characterized by the production rates of particular physical processes, defined as

$$
\mathcal{R}=\sigma \mathcal{L}
$$


where $\sigma$ is the cross section and $\mathcal{L}$ is the luminosity.

Cross section in particle physics experiments is an expression of the likelihood for effective interactions between particles. It is derived from classical picture of collisions and hence in unit of geometrical area. In particle collisions, cross section represents the occurrence probability of certain physical process per unit flux. For instance, the NLO calculations give the cross section of $W H\left(m_{H}=115 \mathrm{GeV}\right)$ production at the Tevatron, $\sigma(p \bar{p} \rightarrow W H)=0.1855 \mathrm{pb}[34]$.

The luminosity at the Tevatron describes the interactions of two beams in terms of particle numbers per unit area per unit time. It is defined as

$$
\mathcal{L}=\frac{f n N_{p} N_{\bar{p}}}{2 \pi\left(\sigma_{p}^{2}+\sigma_{\bar{p}}^{2}\right)} F\left(\frac{\sigma_{l}}{\beta^{*}}\right)
$$

where $N_{p}$ and $N_{\bar{p}}$ are the number of particles in each bunch, $\mathrm{f}$ is the revolution frequency, $\mathrm{n}$ is the number of bunches in either beam. The denominator $2 \pi\left(\sigma_{p}^{2}+\sigma_{\bar{p}}^{2}\right)$ stands for the cross sectional area of the two beams, where $\sigma_{p}$ and $\sigma_{\bar{p}}$ are the widths of the beams which are in Gaussian shapes. The form factor $F\left(\frac{\sigma_{l}}{\beta^{*}}\right)$ depends on the bunch length $\sigma_{l}$ and the beta function $\beta^{*}[10]$. The peak luminosity of RunII at the Tevatron currently is $3 \times 10^{32} \mathrm{~cm}^{-2} \mathrm{~s}^{-1}[35]$.

The instantaneous luminosity is changing over time but we are interested in the number of occurrences for a particular physical process during a period. Thus the integrated luminosity is introduced as the integral of the luminosity over time $\mathcal{L}_{\text {int }}=\int_{t_{0}}^{t_{0}+\Delta t} \mathcal{L} d t$. i.e. Given $1000 \mathrm{pb}^{-1}$ integrated luminosity, the total number of occurrences for $W H\left(m_{H}=115 G e V\right)$ production at the Tevatron during this period is expected to be around $0.1855 \times 1000=185.5$. 


\subsection{The Upgraded DØ Detector}

\subsubsection{Overview of the $\mathrm{D} \emptyset$ Detector}

The $\mathrm{D} \varnothing$ detector is a general purpose particle detector capable of studies on various aspects of the high energy physics of $p \bar{p}$ collisions at the Tevatron. A broad range of topics in particle physics are covered at the $\mathrm{D} \varnothing$ experiment such as search for the Higgs boson, top quark physics, bottom quark physics, precision measurements of electroweak parameters, jet production studies and new physics beyond the Standard Model.

The D $\varnothing$ detector is integrated with various of sub-detector systems for identifying the secondary particles from $p \bar{p}$ collisions. The detector mainly consists of the central tracking system, calorimeter system, muon detector and luminosity monitor as well as forward proton detector and those complex electronics readout systems [11]. As shown in Fig. 3.6, the innermost part is the central tracking system which tracks the charged particles traveling through its 2 Tesla solenoidal magnetic field. The calorimeter system surrounding the central tracking system is made of depleted uranium, copper and stainless steel filled with liquid argon. It measures the energies of the electromagnetic particles and hadronic jets. The outermost is the muon detector made of scintillator counters, drift tubes and toroid magnets. It identifies the muons, which easily penetrate through all the inner detectors without losing much of their energies. All the sub-detector systems are coordinated and read out through complex electronic systems.

As shown in Fig. 3.6, the $\mathrm{D} \varnothing$ detector uses a right-handed Cartesian coordinate system with the origin centered in the detector. The $z$ axis is along the direction of the proton beam which circulates clockwise in the Tevatron ring. The $y$ axis points upward from the Tevatron ring while the $x$ axis points outside of the ring. Thus the 


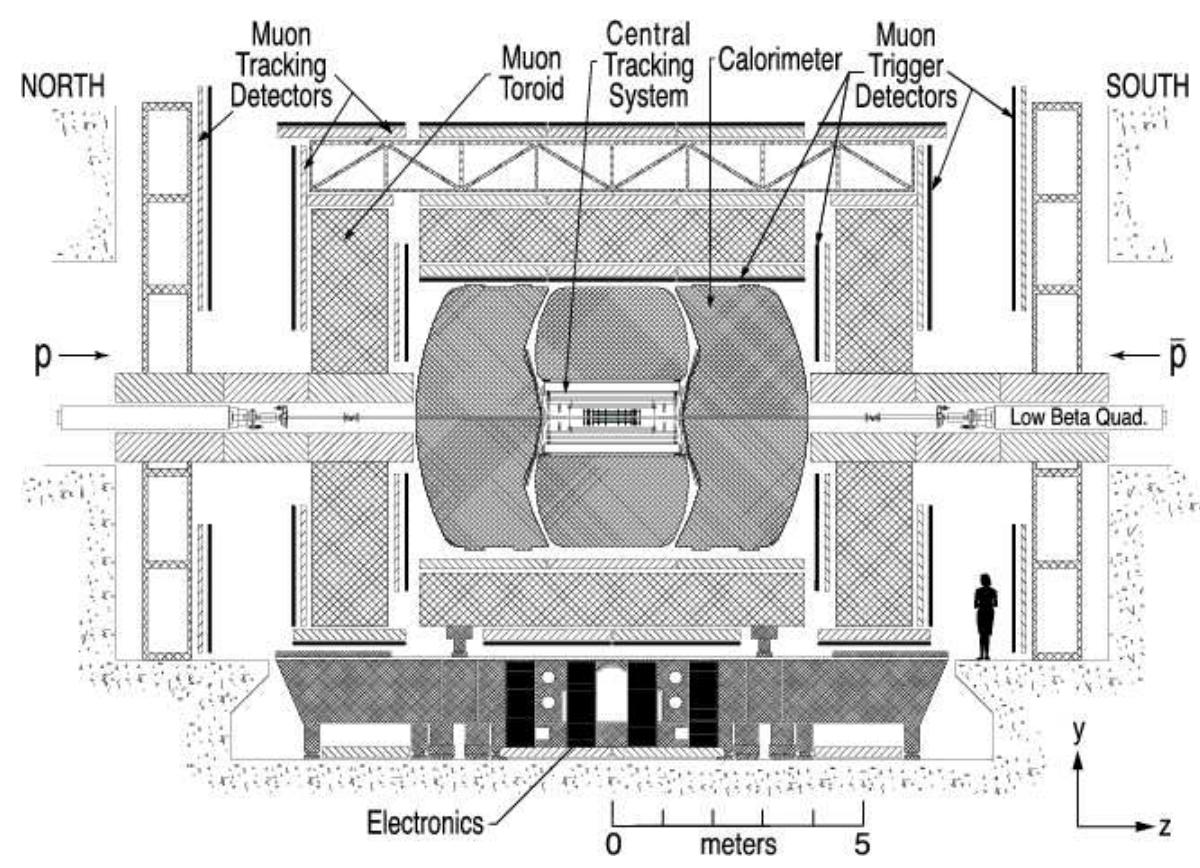

Figure 3.6: Overview of the upgraded D0 detector from inside the Tevatron ring. The forward proton detector is not shown. The details of the central region are shown in Fig. [11]

transverse and longitudinal planes are defined by $x-y$ axis and $z$ axis respectively.

Since the $\mathrm{D} \varnothing$ detector has a somewhat cylindrical shape but the particle collisions are roughly spherically symmetric around the interaction point, the $\mathrm{D} \varnothing$ detector more often employs a combination of cylindrical and spherical coordinate systems, denoted as $(z, r, \theta, \phi)$.

The polar angle $\theta$ is often replaced by the pseudorapidity $\eta$, which approximates rapidity $y$ in relativity theory. The rapidity of a particle is defined as

$$
y=\frac{1}{2} \ln \left(\frac{E+p_{z}}{E-p_{z}}\right)
$$

where $E$ and $p_{z}$ are the energy and longitudinal momentum of the particle. The number of particles produced in given range of rapidity is invariant under Lorentz boost along the $z$ direction. As the energy of the particle is sufficiently large to travel close to the speed of light, the energy is approximately equal to the momentum $E \approx p$.

$$
y \approx \frac{1}{2} \ln \left(\frac{p+p \cos \theta}{p-p \cos \theta}\right)=-\ln \left(\tan \frac{\theta}{2}\right) \equiv \eta
$$


In the high energy case, the pseudorapidity $\eta$ is a good approximation of rapidity. The solid angle region at the $\mathrm{D} \emptyset$ detector is often defined as $\Delta R \equiv \sqrt{\Delta \eta^{2}+\Delta \phi^{2}}$, which is also approximately invariant under Lorentz boosts along the $z$ direction. These coordinates are frequently used in describing the geometric and kinematic variables in this thesis.

\subsubsection{Tracking System}

The DØ central tracking system is necessary for almost all of the physics studies involved with charged particles in the final states, including the searches for the Higgs boson. It measures the trajectories, charge signs and momenta of the particles passing through different components of its detectors. The working principle is that the charge sign and momentum of a charged particle can be precisely determined from its movement in a known magnetic field. The charge sign is determined from the direction in which the particle is deflected. The momentum is calculated through the curvature of the particle path, $k=\frac{q B}{p}$, where $B$ is the magnetic field strength, $q$ and $p$ are the charge and momentum of the particle respectively.

As shown in Fig. 3.7, the central tracking system chief components are the Silicon Microstrip Tracker (SMT), the Central Fiber Tracker (CFT) and the Solenoid. The SMT detector surrounds the $\mathrm{D} \varnothing$ beryllium beam pipe and is made of silicon sensors. It provides a high precision measurement of the tracks and interaction vertices of the particles. The CFT is outside of the silicon tracker and made of scintillating fibers. The position and momentum of the charged particles in the central detector region can be determined by CFT. The solenoidal magnets, between the CFT and the preshower detectors, provides a 2 Tesla uniform magnetic field for the entire tracking system. The entire tracking system is able to locate the interaction vertex with a resolution of $35 \mu \mathrm{m}$ along the beamline, and better than $15 \mu \mathrm{m}$ in the $r-\phi$ 
plane when the transverse momentum of the particle is larger than $10 \mathrm{GeV}$ at $\eta=0$. The momentum resolution that can be achieved is $\left(2+0.15 p_{T}\right) \%$, where $p_{T}$ is the transverse momentum of the particle and in unit of $\mathrm{GeV}$.

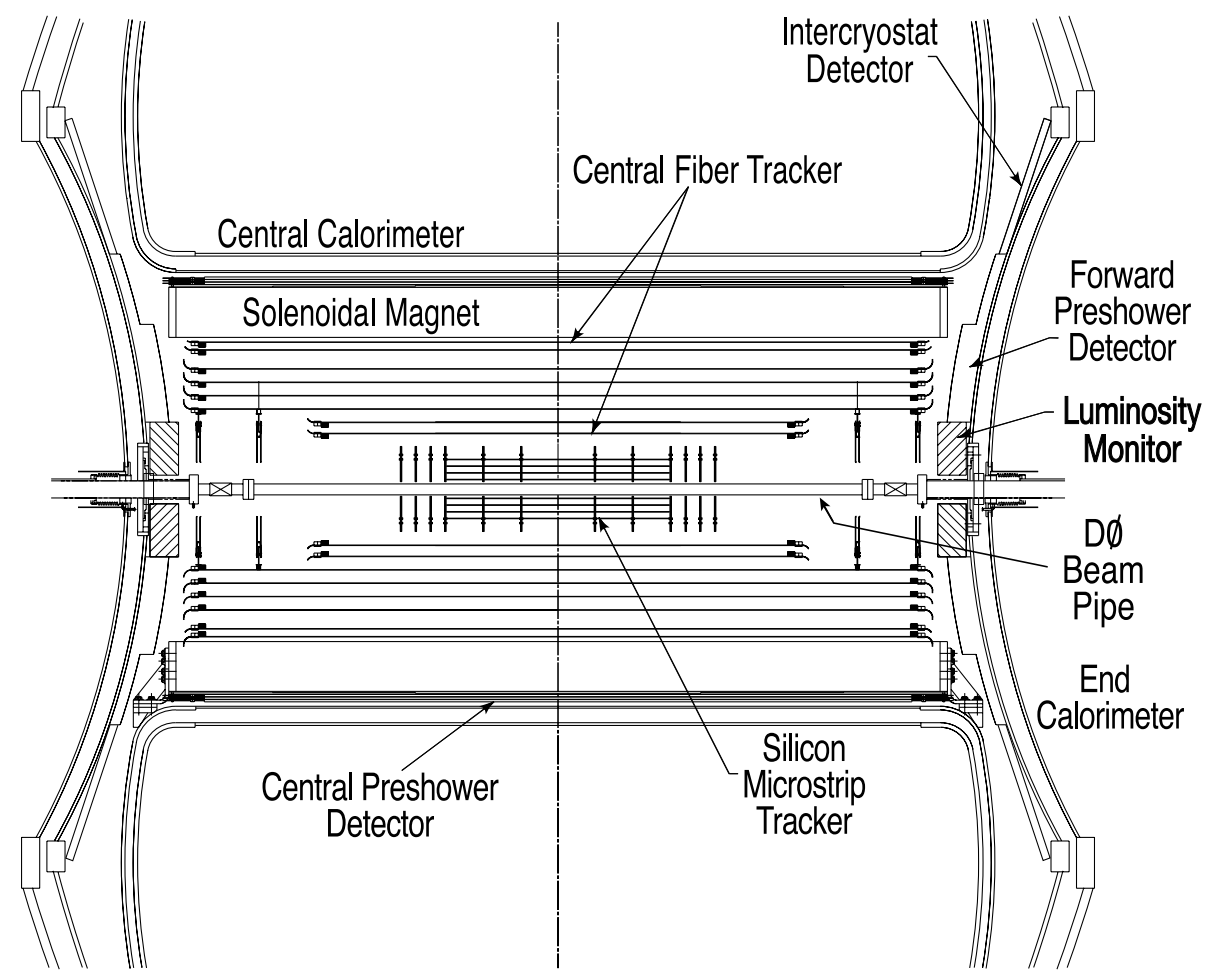

Figure 3.7: A side view of the D0 central tracking detectors in the $x-z$ plane. The central tracking detectors include the SMT, CFT and Solenoid outside the beam pipe. The luminosity monitor, preshower detectors and parts of the calorimeters are also shown in the diagram. [11]

\section{Silicon Microstrip Tracker}

The SMT detector is designed to find tracks and identify their vertices around the colliding region. Its high precision comes from employing silicon detectors, of which the functional units are the $p-n$ junction diodes. The diodes are made of silicon strips with one side n-doped and the other side p-doped. As a charged particle passes through the strips, ionization effects create electron hole pairs and cause a current pulse, which can be recorded by the electronic readout system. When numerous strips are arranged around the nominal interaction points, the trajectories 
of the particles can be precisely determined. The SMT is able to achieve a spatial resolution of $10 \mu m$ in $r-\phi$ plane and $35 \mu m$ in $z$ direction.

The 3D tracking ability of the SMT is reached through its design of combining barrel and disk modules. As shown in Fig. 3.8, there are in total 6 barrels with strips parallel to the beam direction and 16 disks with strips perpendicular to the beam direction. On each side of the origin are 3 barrels, of which each one consists of 4 concentric layers of silicon wafers. The barrels are able to provide better $\phi$ information and cover detector region $|\eta|<2.4$. There are 6 of the F-disks, perpendicular to the beamline, positioned between the barrels. Together with the other 6 F-disks and 4 H-disks, the disk modules provide better measurements on the $z$ direction. During the upgrade of DØ detector in 2006, a new layer, called Layer 0, was added inside the 4 existing layers of silicon wafers, while the forward H-disk was removed.

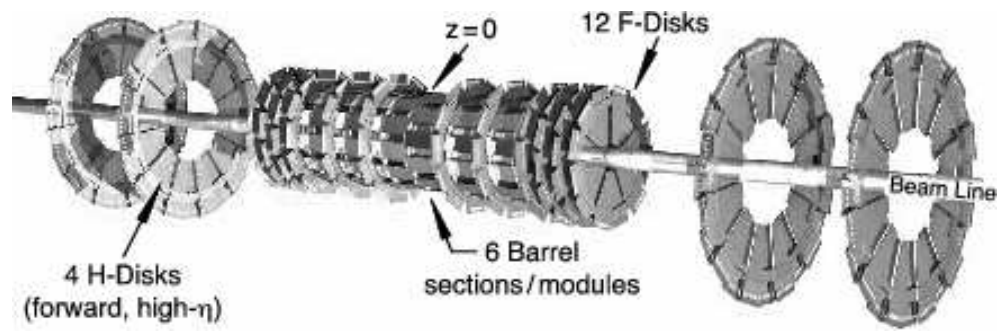

Figure 3.8: The layout of the D0 Silicon Microstrip Tracker with its disk and barrel design. [11]

Note that the geometric scale of the SMT is in large part ruled by the beam collision region, of which $\sigma$ in $\mathrm{z}$ direction is around $25 \mathrm{~cm}$. The most distant F-disk is positioned at $|z|=121.0 \mathrm{~cm}$ with radius $r=26 \mathrm{~cm}$. In general the $\mathrm{SMT}$ is designed to cover detector region $|\eta|<3.0$, which is nearly full $\eta$ coverage of the calorimeter and muon systems. It has up to 792,576 readout channels and requires complicated electronics and high voltage supplies. 


\section{Central Fiber Tracker}

The Central Fiber Tracker [36] tracks charged particles using scintillating fibers. The base core material of the scintillating fibers is polystyrene doped with organic fluorescent dyes. When ionizing particles deposit parts of their energies in the doped polystyrene, molecular excitations and rapid fluorescence decays will occur, emitting $\sim 540 \mathrm{~nm}$ light. The light is then extracted through clear fiber waveguides and directed to visible light photon counters (VLPCs), which are avalanche photodetectors and convert the light to electric signal quickly and efficiently.

The CFT is designed to cover the detector region $|\eta|<2.0$ as shown in Fig. 3.9. It consists of 8 layers of concentric cylinders with radius ranging from $19.5 \mathrm{~cm}$ to $51.5 \mathrm{~cm}$. Each layer is actually composed of two sub-layers, one with fibers oriented parallel to the beam direction (called axial layer) and the other with a stereo angle of $+3^{o}$ or $-3^{o}$ (called stereo layer $u$ or $v$ ). From the innermost to the outermost, the scintillating fiber layers are laid out as $z u-z v-z u-z v-z u-z v-z u-z v$. The fiber has a diameter of $835 \mu \mathrm{m}$ and thus determines the inherent resolution of doublet layer to be around $100 \mu \mathrm{m}$. The CFT in total has $\sim 76,800$ readout channels and is finally combined with the SMT to provide tracking information of charged particles. Note that the signals from the axial doublet layers also serve as the fast trigger decisions, based on the number of tracks above certain transverse momentum thresholds such as $1.5 \mathrm{GeV}$.

\section{The Solenoid}

The Solenoid is designed for momentum measurement and track recognition by bending the paths of charged particles. It provides the SMT and CFT with a uniform magnetic field of $\sim 2$ Tesla with either polarity along the direction of beamline, as 


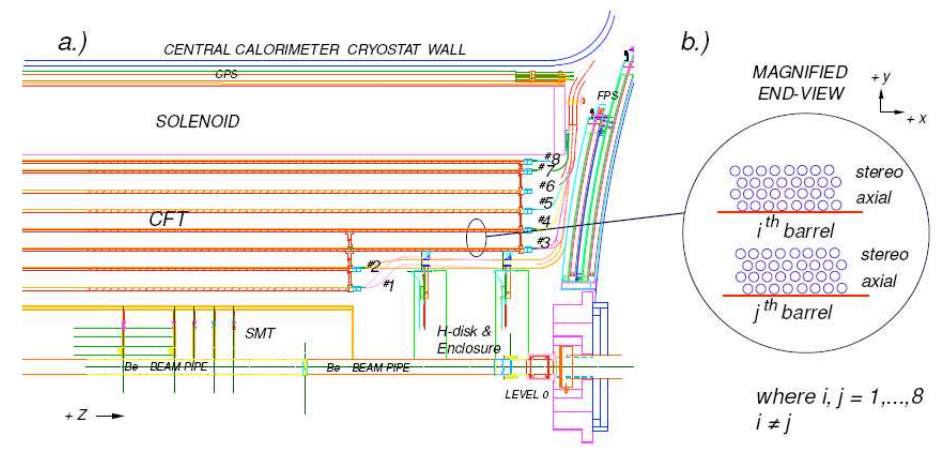

Figure 3.9: Schematic view of the D0 Central Fiber Tracker. a) A side view of the CFT in $r-z$ plane. It is located between the SMT and the Solenoid. There are 8 layers of concentric barrels labeled as \#1 to \#8 from the innermost the outermost. b) A magnified end view of the barrels in $x-y$ plane. Each barrel contains an axial doublet layer along the beam direction and another steoreo doublet layer at a stereo angle of $+3^{\circ}$ or $-3^{\circ}$.

shown in Fig. 3.10. Its superconducting coils carry up to $4749 \mathrm{~A}$ current and are cooled to $4.6 \mathrm{~K}$ using liquid helium. The physical size is limited by the space within the calorimeters to be $2.73 \mathrm{~m}$ in length and $1.42 \mathrm{~m}$ in diameter.

\section{The Preshower Detectors}

The existence of the Solenoid could degrade the energy resolution of the calorimeters, since the solenoidal materials interact with particles which would shower before reaching the calorimeters. The Preshower detectors are introduced to improve the energy measurement particularly for electrons and photons, as well as enhance the sensitivity of the tracking system. The Preshower detectors also use scintillating fibers while absorber materials are attached to initiate the electromagnet showers. As can be seen in Fig. 3.7, there are Central Preshower Detector (CPS) and Forward Preshower Detector (FPS), which cover the detector region $|\eta|<1.3$ and $1.5<|\eta|<2.5$ respectively. The CPS is sandwiched in a $\sim 5 \mathrm{~cm}$ space between the Solenoid and the Calorimeter. It is made of 3 layers of axial and stereo fiber strips with approximately 1 radiation length lead radiator, which together with the solenoidal materials make $\sim 2$ radiation lengths. The FPS functions similarly al- 


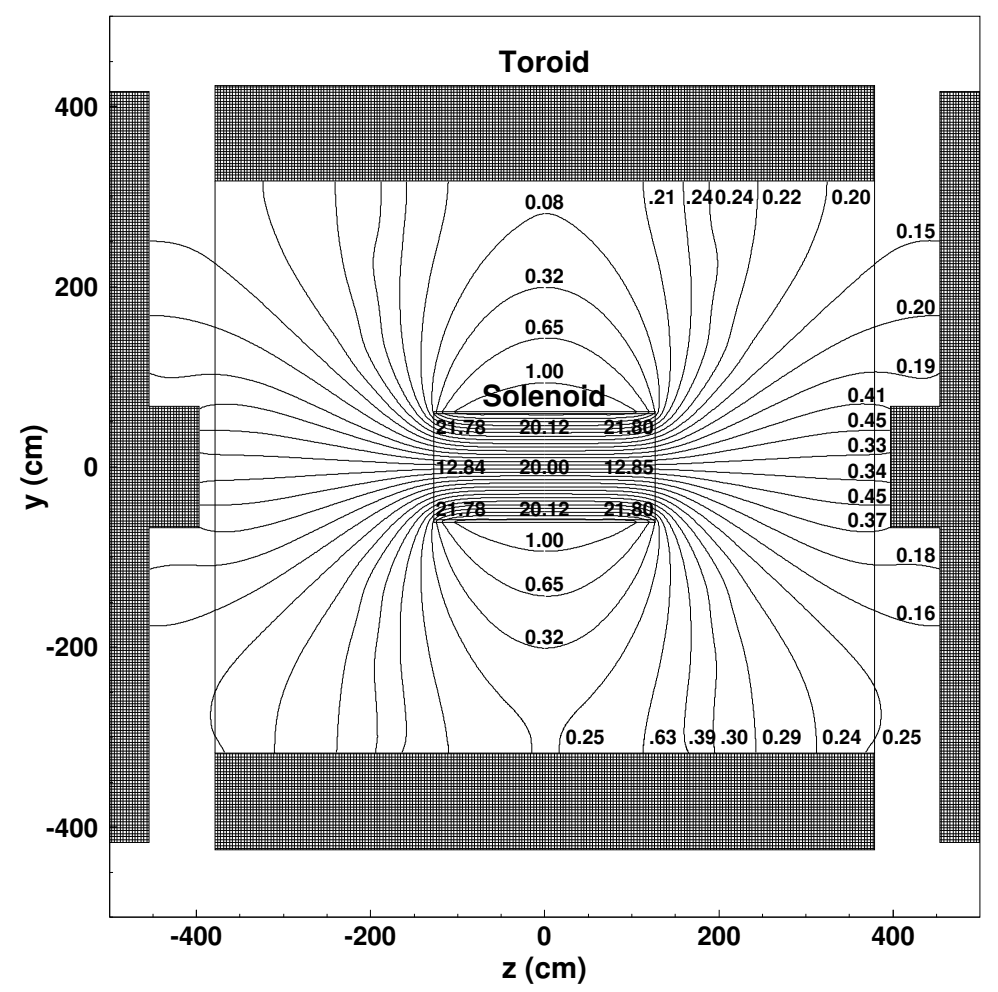

Figure 3.10: The magnetic field lines in $y-z$ plane of the $\mathrm{D} \varnothing$ magnetic system. The field strengths of both solenoidal and toroidal magnets are in unit of kiloGauss ( 1 kiloGauss $=0.1$ Tesla), at full current $4749 \mathrm{~A}$ and $1500 \mathrm{~A}$ respectively. The field in the central toroid is $\sim 1.8 T$, while it is $\sim 1.9 T$ in the end toroid. [11]

though its design is different. Note that the preshower information is also included in the Level 1 trigger because these detectors provide fast energy and position measurements.

\subsubsection{Calorimeter System}

The DØ Calorimeter system measures energy depositions and shower shapes of electrons, photons and jets through their electromagnetic and hadronic showers in the absorber materials. Electromagnetic shower refers to the process that when electrons or photons enter the absorber materials, they develop a cascade of secondary particles via electron pair production $\left(\gamma \rightarrow e^{+} e^{-}\right)$and bremsstrahlung $(e \rightarrow e \gamma)$. Deeper into the materials, the number of secondary particles increases while the energies of the original incident particles decrease exponentially as $E=E_{0} e^{-x / X_{0}}$, where $x$ is the 
depth of the particle traveling into the material and the $X_{0}$ is the radiation length. Hadronic shower is similar but occurs via strong nuclear interaction processes. The secondary particles are mainly pions $\pi^{0}$ and $\pi^{ \pm}$. The $\pi^{0}$ converts to a photon pair $\left(\pi^{0} \rightarrow \gamma \gamma\right)$ and $\pi^{ \pm}$successively interact with the material via strong forces. The corresponding strong interaction length $\lambda_{0}$ is typically much larger than radiation length $X_{0}$.

The $\mathrm{D} \varnothing$ calorimeter system is designed to be sampling calorimeters consisting of calorimeter readout cells. A typical cell contains an absorber plate and a signal board with gaps filled with liquid argon. As shown in Fig. 3.11, the absorber plate is made of depleted uranium and electrically grounded, while the copper signal board is highly insulated and applied a high voltage of $\sim 2.0 \mathrm{kV}$. As the particles shower in the absorber plate, they produce secondary particles which ionize the argon atoms. The resulting electrons drift across the gap in the electric field and are collected by the signal board. The cells are aligned to form different layers, which in turn are radially grouped to form readout towers with $\Delta \eta \times \Delta \phi$ of $0.1 \times 0.1$.

Note that the drift time of electrons in the calorimeter cell gap is $450 \mathrm{~ns}$, as shown in Fig. 3.11. It would not allow a complete signal charge integration, since there are beam crossings every 396ns in the RunII of Tevatron. The deficiencies are remedied by the electronics readout system with a combination of preamplifiers, signal shaping and sampling. The calorimeter signals are also included in the Level 1 trigger decisions.

The $\mathrm{D} \varnothing$ calorimeter system is built into Electromagnetic Calorimeters, and Hadronic Calorimeters which are further divided to Fine Hadronic and Coarse Hadronic ones. As shown in Fig. 3.12, the inner Electromagnetic Calorimeters are designed to be 4 thinner layers with absorbers $\sim 20 X_{0}$ in total, so as to absorb the electrons and 
photons but let hadronic jets pass through. The Hadronic Calorimeters consist of much larger modules with absorber materials ranging more than $6 \lambda_{0}$.

The geometric size of the $\mathrm{D} \varnothing$ Calorimeter is able to cover the detector region of $|\eta|<4.0$. A detailed view is shown in Fig. 3.13, the Central Calorimeter (CC) covers detector region up to $|\eta|=1.1$ while the End Calorimeter (EC) covers the region of $1.5<|\eta|<4.0$. The gap of range $1.1<|\eta|<1.4$ is the region between the central and end cryostat systems called the inter-cryostat region (ICR). In order to sample the energies of the particles in the ICR region, a pair of InterCryostat Detectors (ICD) made of scintillating materials are attached to the surfaces of the end cryostats.

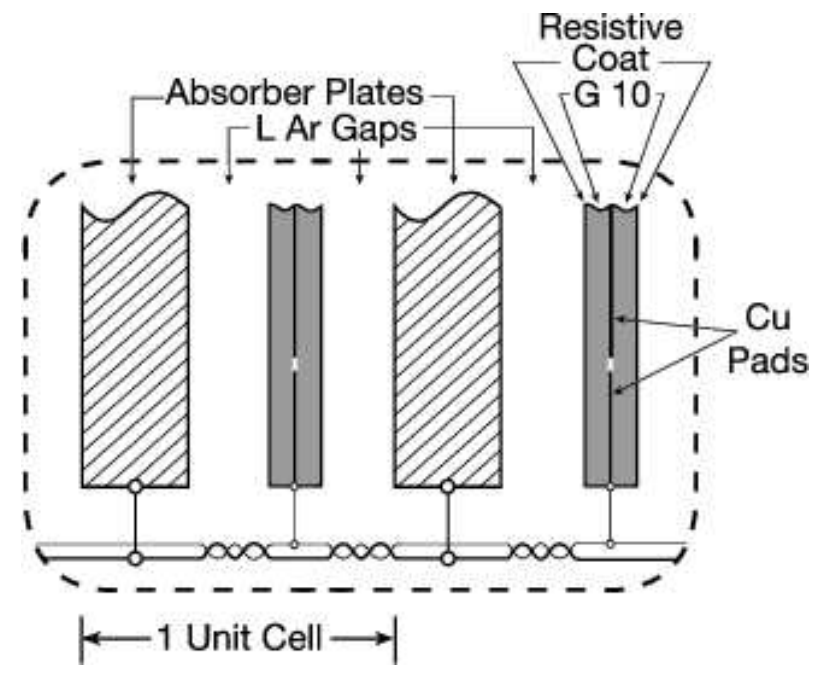

Figure 3.11: Schematic view of unit cell for the calorimeter. A unit cell includes the absorber plate and signal board with gaps filled with liquid argon. The absorber plate is made of depleted uranium, while the signal board is copper pad insulated with G10 insulator and coated with highly resistive epoxy. A high voltage of typically $2 \mathrm{kV}$ is applied between the signal board and the absorber plate. The liquid argon gap is $2.3 \mathrm{~mm}$ and takes the electrons about $450 \mathrm{~ns}$ to drift across to the signal board. [11]

\subsubsection{The Muon System}

The muon system [37] of the DØ detector is designed to efficiently identify muons and measure their momenta. Compared to electron, muon is $\sim 200$ times heavier 


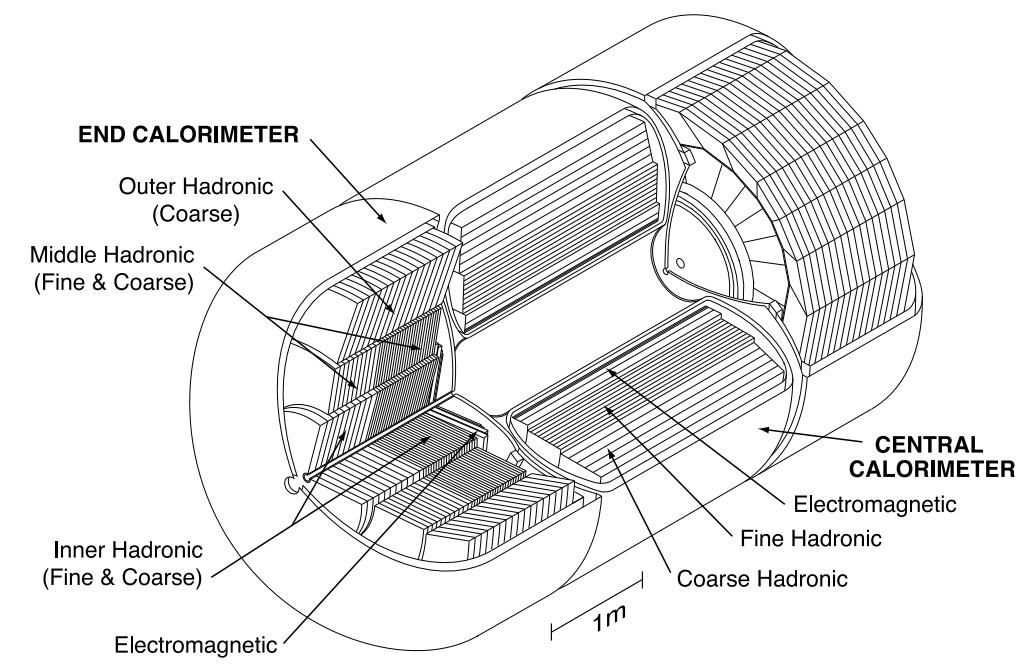

Figure 3.12: Isometric view of the central and two end calorimeters. Electromagnetic modules have 4 layers with absorber materials of approximately 1.4,2.0,6.8, $9.8 X_{0}$ in the central and 1.6,2.6,7.9,9.3 $X_{0}$ in the end. In the central region, the hadronic modules have $1.3,1.0,0.76 \lambda_{0}$ for the fine ones and $3.2 \lambda_{0}$ for the coarse part. In the two end regions, the absorb depths are $4 \times 1.1+4.1 \lambda_{0}$ for the inner hadronic, $4 \times 0.9+4.4 \lambda_{0}$ for the middle hadronic and $6.0 \lambda_{0}$ for the outer hadronic. [11]

and has less ionizing and bremsstrahlung effects. Muon is able to easily penetrate through the inner tracking and calorimeter systems without losing much of its energy. As shown in Fig. 3.6, the $\mathrm{D} \varnothing$ muon system is built right outside of the calorimeter system. The central muon detector is capable of detecting muon in the detector region of $|\eta|<1$ while the forward muon detector covers $1<|\eta|<2$. The entire system is a combination of drift tubes, scintillation counters and toroidal magnets.

The tracking ability of the muon system is provided by the proportional drift tubes (PMT) and mini drift tubes (MDT). There are 94 PDT chambers grouped to $\mathrm{A}, \mathrm{B}$ and $\mathrm{C}$ layers in the central detector region. The inner A-layer is attached on the inside surfaces of the central toroidal magnet while B-layer and C-layer are outside of the toroidal magnets. The PDT chambers are filled with mixture of gases (84\% argon, $8 \% \mathrm{CF} 4$ and $8 \% \mathrm{CH} 4$ ) with maximum electron drift time of $450 \mathrm{~ns}$. The MDTs are also grouped into A, B and $\mathrm{C}$ layers in the forward detector region. The smaller MDT is filled with gas mixture of (90\% CF4 and 10\% CH4) and has electron 


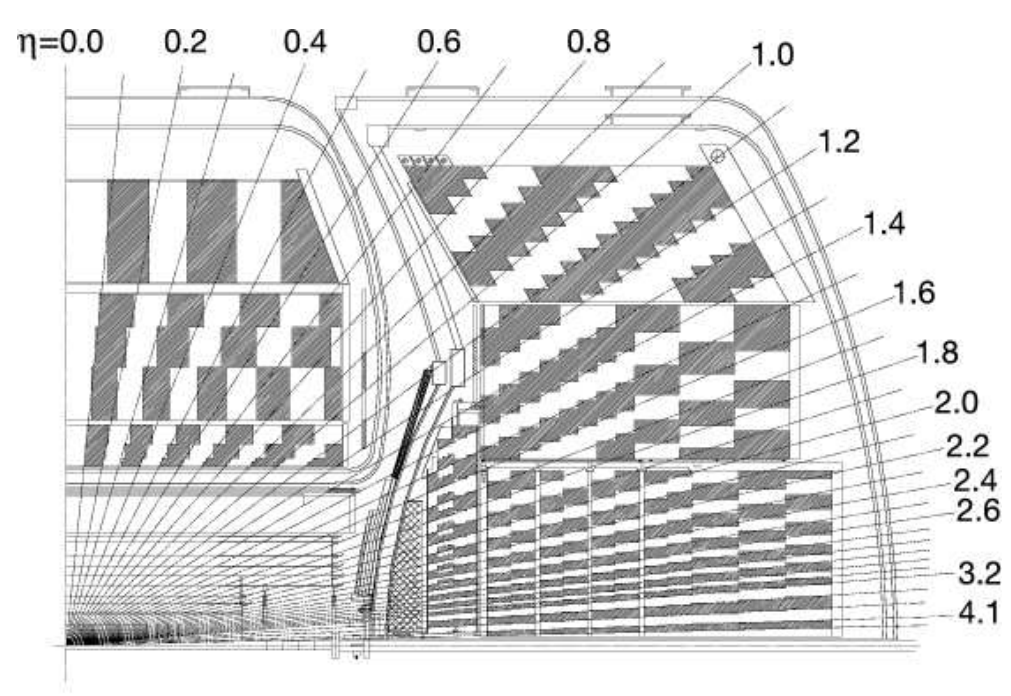

Figure 3.13: Schematic view of a portion of the $\mathrm{D} \varnothing$ calorimeters in $r-z$ plane. The CC covers up to $|\eta|=1.1$ detector region, while the EC covers the region of $1.5<|\eta|<4.0$. The ICR region of $1.1<\mid$ eta $\mid<1.4$ is covered by ICD. [11]

drift time less than $132 \mathrm{~ns}$. The cordinate resolution is $\sim 1 \mathrm{~mm}$.

The scintillation counters are added to trigger on muon with good time resolution. In the central detector region, there are 2 layers of scintillation counters, $A \phi$ counters inside the toroidal magnets and outer counters in the most outside. In the forward region, the 3 layers of scintillation counters are mounted close to each layer of MDT chambers. These counters are associated with proton-antiproton collision with precise time information. Thus it is used to trigger on muons from $p \bar{p}$ collisions as well as reject cosmic ray muons. The time resolution is $\sim 2 \mathrm{~ns}$.

The iron toroidal magnets are built to provide a local muon momentum measurement. There is one central toroidal magnet as well as two end toroidal magnets with square annulus of $109 \mathrm{~cm}$ thickness. The magnetic field is $1.8 T$ with a current of $1500 A$ in the coils. The momentum resolution of muon is dominated by the central tracking system up to $100 \mathrm{GeV}$. The forward muon system improves the resolution of muon with higher momentum, especially for tracks in the detector region 
$1.6<|\eta|<2.0$

\subsubsection{Luminosity Monitor, Trigger System and Data Acquisition System Luminosity Monitor}

The luminosity at the Tevatron is discussed in Section 3.1.2. The DØ luminosity monitor is used to measure the luminosity of $p \bar{p}$ collisions as well as the $z$ position of the interaction vertex. As shown in Fig. 3.14, the luminosity detector is composed of two discs mounted at $z= \pm 140 \mathrm{~cm}$ along the beamline and inside the end calorimeters. Each disc is made of 24 plastic scintillation counters with PMT attached to read out the signal. The time-of-flight resolution of the counters is about $0.3 n s$. The gain of fine mesh PMTs (Hamamatsu R5505Q) is reduced by a factor of 30 because they are exposed to $\sim 1 T$ solenoidal magnetic field. The luminosity monitor is capable of covering the detector region of $2.7<|\eta|<4.4$. [12]

The instantaneous luminosity is determined by measuring the rate of inelastic $p \bar{p}$ collisions, as given by

$$
\mathcal{L}=\frac{1}{\sigma_{p \bar{p}, e f f}} \frac{d N_{p \bar{p}}}{d t}
$$

where $\sigma_{p \bar{p}, \text { eff }}$ is effective cross section of inelastic $p \bar{p}$ collision by taking into account of acceptance and recording efficiency. The $z$ position of interaction vertex can be reconstructed by luminosity detectors based on the information of time-of-flight difference,

$$
z_{v}=\frac{c}{2}\left(t_{-}-t_{+}\right)
$$

where $t_{-}$and $t_{+}$are the time of flight for the particles from the interaction point to the two detectors at $z=\mp 140 \mathrm{~cm}$. The Gaussian width $\sigma_{z}$ of the $p \bar{p}$ beam collisions is approximately $30 \mathrm{~cm}$. Thus a requirement of $\left|z_{v}\right|<100 \mathrm{~cm}$ is applied to select the inelastic beam collisions and reject the beam halo backgrounds. Fig. 3.15 is an 


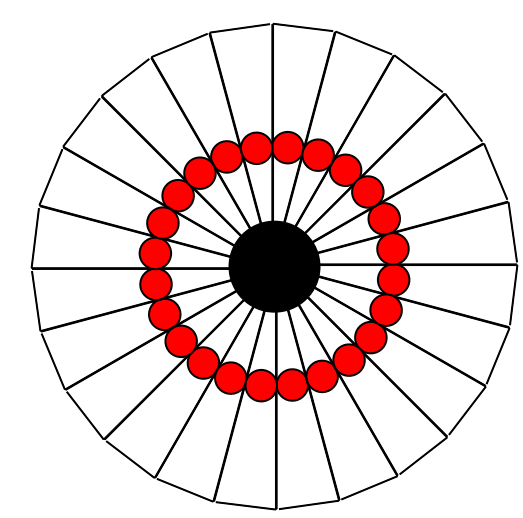

(a) $r-\phi$ view

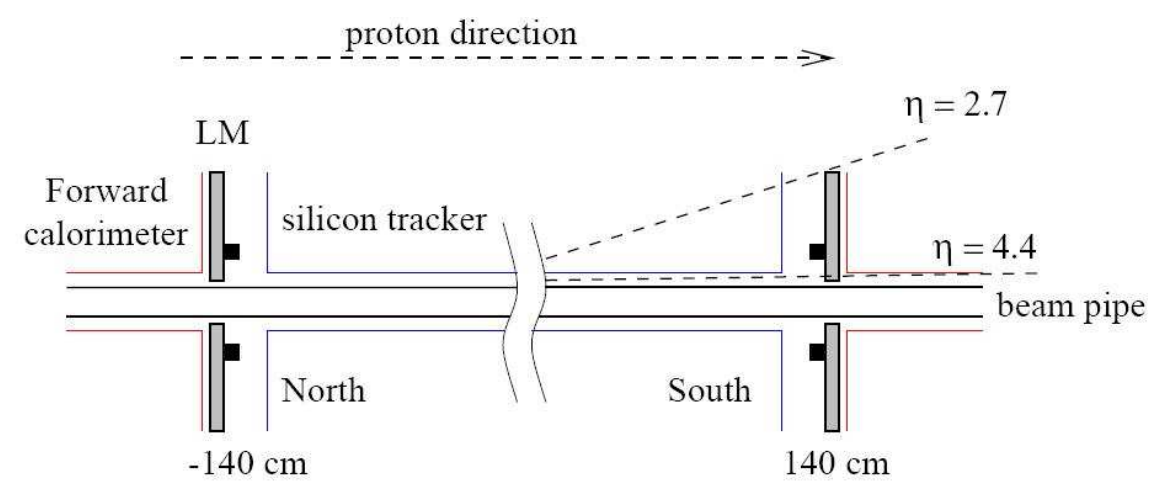

(b) $r-z$ view

Figure 3.14: Diagrams of the D $\varnothing$ Run II Luminosity Monitor. a) $r-\phi$ view of the monitors. The wedge shaped are the the scintillation tiles, on which the small circles are the corresponding photomultiplier tubes. b) $r-z$ view of the monitors. The two monitors are positioned at $z= \pm 140 \mathrm{~cm}$ along the beamline. [12]

illustration of the integrated luminosity for the data accumulated by the $\mathrm{D} \varnothing$ detector as a function of time. So far the $\mathrm{D} \emptyset$ detector has recorded an integrated luminosity of $6.1 \mathrm{fb}^{-1}$ out of total $6.9 \mathrm{fb}^{-1}$ delivered by the Tevatron.

\section{Trigger System}

The $\mathrm{D} \varnothing$ trigger system is an online decision system designed to select and store the physics events of interest from hadron collisions. With the beam bunch crossing time of 396ns and peak instantaneous luminosity $\sim 3 \times 10^{32} \mathrm{~cm}^{-2} \mathrm{~s}^{-1}$, the proton and 


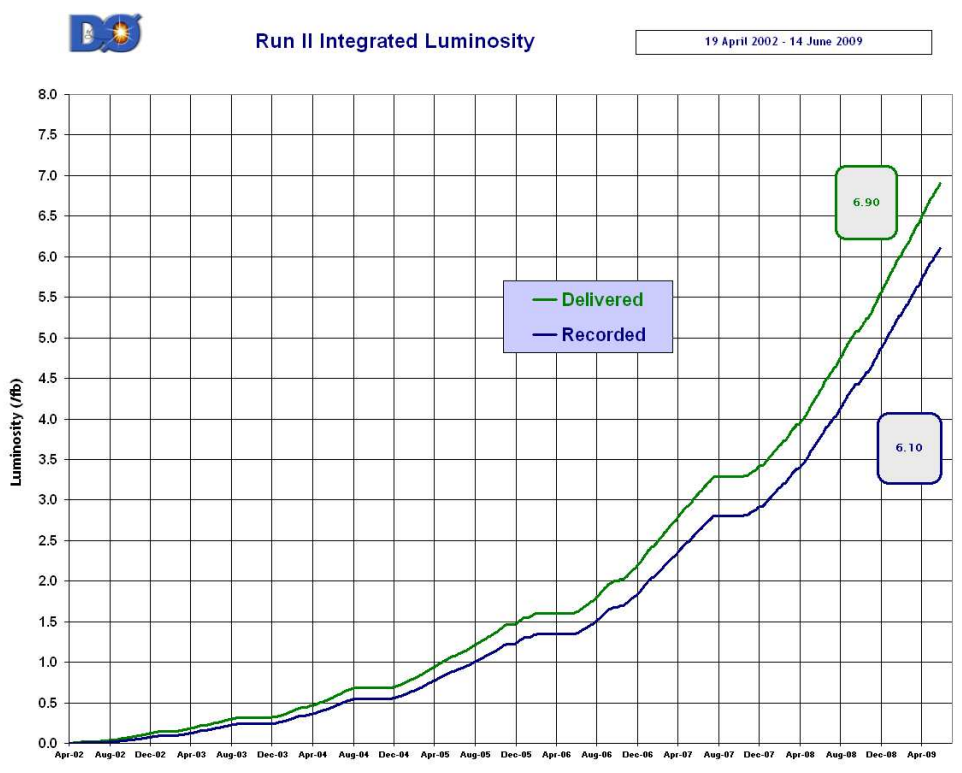

Figure 3.15: The total integrated luminosity. The lower curve is the luminosity recorded by the $\mathrm{D} \varnothing$ detector, while the upper curve is the luminosity delivered by the Tevatron.

antiproton beams at the Tevatron are able to collide at $\sim 2.5 \mathrm{MHz}$. However, most of the events are background not the desired physics processes with high transverse momentum which occur at small probabilities. In order to reject the overwhelming background and cut the cost of data storage and bandwidths, the $\mathrm{D} \varnothing$ trigger system is built based on the readout from subdetectors and grouped into three levels. As shown in Fig. 3.16, the lower level decisions are fed into the higher lever decisions with more complexity. Given the original data of the detector is at $1.7 \mathrm{MHz}$, the Level 1 (L1) trigger typically reduces the rate to $\sim 2 k H z$. The output of the L1 trigger is fed as input to the Level 2 (L2) trigger with an output of $500 \sim 1000 \mathrm{~Hz}$, which in turn is further processed by Level 3 (L3) at $\sim 50 k H z$. The events that pass the L3 trigger decisions are finally recorded to tape for offline reconstruction.

The Level 1 trigger is hardware based with its simple algorithms implemented using field programmable gate arrays (FPGAs). It examines every $p \bar{p}$ collision event and determine if it is worth feeding into the Level 2 trigger decisions. The response 


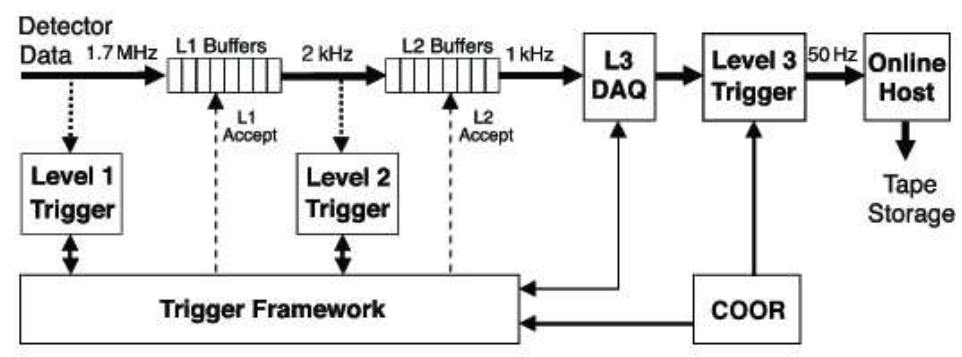

Figure 3.16: An overview of the data flow in the DØ trigger and data acquisition systems. [11]

time of $\mathrm{L} 1$ trigger is $3.5 \mu \mathrm{s}$ or less and the rate is limited by the readout deadtime. These quick decisions are reached by using part of the raw detector information from the luminosity monitor, the central fiber tracker, the calorimeter and the muon system, as shown in Fig. 3.17. The L1CAL decision is based on the number of calorimeter towers $(\Delta \eta \times \Delta \phi=0.2 \times 0.2)$ with transverse energy deposition above certain energy thresholds. The L1CTT reconstructs the tracks of charged particles by combining the information from the central fiber tracker and preshower detector. It requires tracks have certain transverse momenta passing the thresholds such as 1.5 $\mathrm{GeV}$ and $5 \mathrm{GeV}$. The L1MUO recognizes muon candidates by using hits from muon scintillation counters and muon wire chambers as well as tracks from L1CTT. It decides on the number of muons with qualified transverse momentum, track quality and detector region.

The Level 2 trigger is partly hardware based and also utilizes software to quickly reconstruct basic physics objects. It uses the output of the L1 trigger as well as the SMT detector information. processor making trigger decisions. As shown in Fig. 3.17, L2CAL, L2PS, L2CTT, L2STT and L2MUO are the L2 preprocessors and L2Global is trigger decision processor. The preprocessors process the information from the subdetector systems. The basic physics objects such as jets, electron, photon, muon, Missing $E_{T}$ and specific tracks are reconstructed and correlated. The 


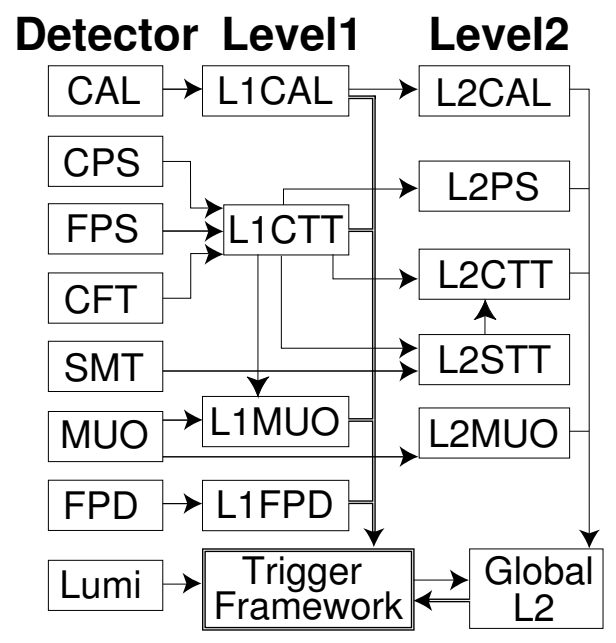

Figure 3.17: Information flow of the DØ Level 1 and Level 2 trigger systems. [11]

L2Global makes the trigger decision based on the physics signatures in the correlations of these physics objects. The L2 trigger is able to handle up to $10 \mathrm{kHz}$ input rates with maximum accepting rate of $1 \mathrm{kHz}$.

The Level 3 trigger is completely software based by running fast reconstruction algorithms on a farm of Linux PCs. Its trigger decision is based on the physics objects or the relations between these objects such as the azimuthal angle between electron and Missing $E_{T}$. The specific software algorithms called filter tools are used to generate these objects and their relations. All the filter tools are controlled by the ScriptRunner, which acts as the interface to the L3 framework. The ScriptRunner calls the filter tools in the order specified by the trigger list until an event passes or fails the criteria. The L3 accepted events are sent to the online host to be recorded.

\section{Data Acquisition System (DAQ)}

The $\mathrm{D} \varnothing$ data acquisition system is designed for data handling and overall running control. As shown in Fig. 3.18, the detector component data is fully digitized by Single Board Computers (SBCs) on the VME crates. When the SBCs receive the instruction information including an unique event identifier from the Routing Master, 
the event fragments are then transported to the L3 farm nodes to be built into a complete event. Once the event passes the L3 trigger filter, it is sent to the online host for data monitoring and storage. The overall running control and detector

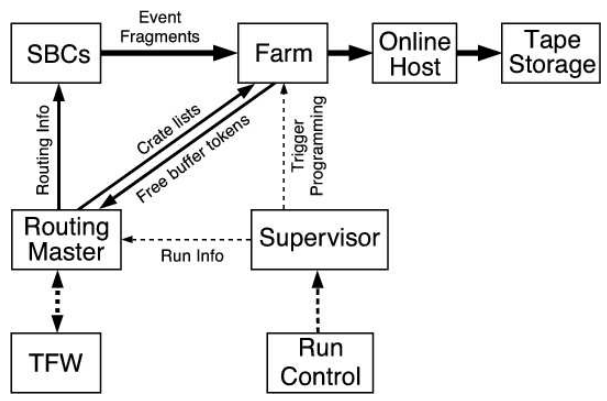

Figure 3.18: Schematic view of the data flow in the DØ L3DAQ system. [11]

configuration is coordinated by the program COOR, which is running on the online host systems. The COOR system receives requests from users and sends commands to the L1, L2, L3 and data logging subsystems. The typical size of a raw data event is $\sim 300 \mathrm{~KB}$. Therefore with a L3 trigger rate of $\sim 50 \mathrm{~Hz}$, the L3DAQ is transferring the raw data to tape storage at a rate of $\sim 15 \mathrm{MB} / \mathrm{s}$. 


\section{CHAPTER IV}

\section{Event Reconstruction}

The raw data chunks of both real data events and simulated events are processed using the DØ Offline Reconstruction Program (RECO) [38] to reconstruct the physical objects. The offline reconstruction algorithms used to identify the physical objects are described in Section 4.1. In order to obtain the true kinematic information of these objects, corrections are applied to data and simulated events, as summarized in Section 4.2. The b jets are identified through the D $\varnothing$ Neural Network b-jet Tagger. The tagging algorithms as well as the corrections in this analysis are introduced in Section 4.3.

\subsection{Offline Event Reconstruction}

The program RECO is a collection of software algorithms and associates the reconstructed objects with data chunks based on the DØ Event Data Model [39]. It is structured in hierachical steps for event reconstruction. First, the raw data chunks are unpacked to decode the raw information, associate electronics readout with detector components and apply specific detector calibration constants. Secondly, the global tracks are reconstructed and put into track chunks using the information of hits

within SMT and CFT detectors. Using tracking information, the primary vertices and displaced secondary vertices are searched for and associated to tracks. Finally 
the track information is combined with the energy depositions in the calorimeter and the preshower detectors as well as the hits in the muon chambers, to identify physical objects such as electron, photon, muon, neutrino and jet candidates.

\subsubsection{Track and Primary Vertex Reconstruction Track Reconstruction}

Charged particles travel in helical paths in the solenodial magnetic fields. The signals originated from the adjacent silicon strips or scintillating fibers are grouped into hit clusters, which are used to find tracks. At the moment there are two patttern recognition algorithms, called the Histogramming Track Finder (HTF) [40] and the Alternative Algorithm (AA) [41], being used to find tracks at DØ.

The HTF algorithm is based on the feature extraction technique known as Hough transform [42]. The trajectory of a charged particle projected onto the transverse plane is circular and charactarized with parameters $\rho, d_{0}$ and $\phi$, where $\rho$ is the curvature $q B / p_{T}, d_{0}$ is the distance of closest approch to the beam spot, and $\phi$ is the direction of the track at $d_{0}$. Each hit in the transverse plane with position $(x, y)$ is transformed to a line in the parameter space $(\rho, \phi)$. All the lines intersect at one point corresponding to the parameters of the trajectory. Each hit increases the histograms by looping over the $\rho$ divisions, where $(\rho, \phi)$ place is divided into cells. The total number of operations is $\sim N_{h} \times N_{\rho}$, where $N_{h}$ is number of hits and $N_{\rho}$ is number of the division. In practice, the first hits are taken from either SMT or CFT independently and duplicate tracks are finally removed.

The AA algorithm is based on the hypothesis that the tracks would first leave hits in the SMT detector. A hypothetical track is built upon three hits in the SMT. The first hit could be any hit in the SMT barrels or F-disks. The second hit has to be within the azimutal window of $\Delta \phi=0.08$ viewed from the beam spot and the first 
hit. The third hit must have a radius of curvature large than $30 \mathrm{~cm}$ (corresponding to $p_{T}>180 \mathrm{MeV}$ ) and an axial impact distance less than $2.5 \mathrm{~cm}$. Then the track hypothesis is extrapolated to the next layers of the tracking system by adding more hits until it violates one of the following requirements: $\chi^{2}<16$, less than three consectively missing and within the detector. All the possible track hypothesises are stored with each track allowed to share at most $2 / 3$ of its hits.

The track candidates from both algorithms are combined by removing duplicated ones and ordering all the candidates. The DØ Kalman track fit [43], is then used for fitting and finding tracks. Thought of as an improved form of multidimensional chisquare minimization, it starts from an initial guess or partially reconstructed track and propagates through the detector by taking into account of magnetic curvature, multiple scattering and energy loss. The Kalman fit algorithm works best when several measurements are added and track parameters are well determined.

\section{Primary Vertex Reconstruction}

The primary interaction vertices of $p \bar{p}$ collisions are reconstructed by extrapolating tracks to their common origins using the adaptive primary vertex algorithm [44]. The reconstruction process mainly involves track selection, vertex fitting and vertex selection.

The tracks are first selected by requiring $p_{T}>0.5 \mathrm{GeV}$ and at least 2 hits in the SMT detector. A z-clustering algorithm is applied to cluster the tracks within $\Delta z=2 \mathrm{~cm}$ for each interaction vertex. The common vertex for each z-cluster is determined using the Kalman filter vertex fitting algorithm. In this process, the fitting is performed iteratively by removing the tracks with the highest $\chi^{2}$ contribution, until the total $\chi^{2} /$ dof is less than 10 . The tracks belonging to each common vertex are then further selected with the criterion $d c a / \sigma_{d c a}<5$, where $d c a$ is the distance 
of closest approach to the beam spot.

The adaptive fitting algorithm is then applied to reduce the contribution of distant tracks to the vertex by assigning weights to the tracks. The track errors are reweighted as a function of their contribution to the $\chi^{2}$ of the vertex fitting, as the following

$$
w_{i}=\frac{1}{1+e^{\left(\chi_{i}^{2}-\chi_{\text {cutoff }}^{2}\right) / 2 T}}
$$

where $\chi_{i}^{2}$ is the $\chi^{2}$ contribution of the track $i, \chi_{\text {cutoff }}^{2}$ is the cutoff value when $w_{i}$ drops to 0.5 and $T$ is control parameter for the sharpness of the function. Starting with the initial values set to 1.0, the weights are calculated iteratively according to formula 4.1 with each iteration derived from the previous one. If $w_{i}<1.0 \times 10^{-6}, w_{i}$ is then set to zero. The calculations are repeated until it satisfies the convergence criteria, which require both $\max \left|w_{i}-w_{i-1}\right|<1.0 \times 10^{-4}$ and iteration number less than 100 .

The probabilistic primary vertex selection algorithm [45] is finally used to identify the primary vertex. The discrimination criteria are based on the fact that the tracks originated from the hard scattering primary vertex tend to have higher $p_{T}$ than those from the minimum bias event vertices, as shown in Fig. 4.1(a). Each track is assigned a probability that it is coming from a minimum bias vertex. The likelihood of each vertex to be a minimum bias vertex can be built from these individual probabilities, illustrated in Fig. 4.1(b). The studies in [46] indicates that the reconstruction efficiency of the primary vertex is nearly $\sim 100 \%$ for both data and simulated events. 


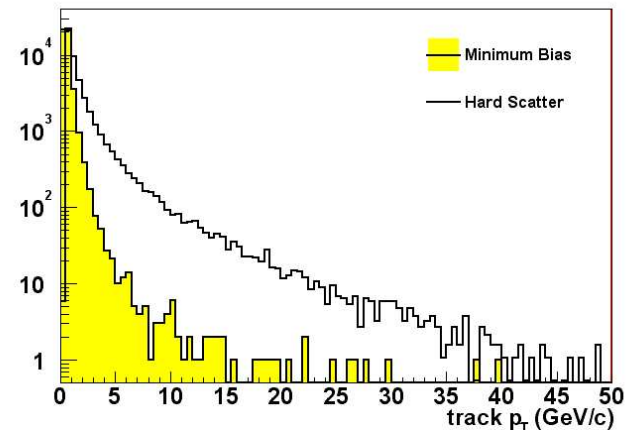

(a) $p_{T}$ spectrum of tracks

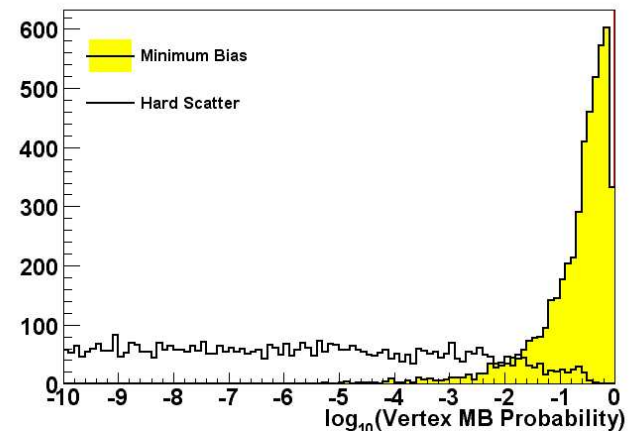

(b) vertex probability

Figure 4.1: a) $p_{T}$ spectrum of tracks from the hard scattering physics data events and the simulated minimum bias events. b) Vertex probability from the hard scattering physics data events and the simulated minimum bias events. [13]

\subsubsection{Electromagnetic Object Reconstruction}

The EM object reconstruction algorithm [47] is based on the fact that the EM objects have narrow shower shapes and deposit most of their energies in the EM calorimeter. The tracks in the central tracking system are matched to the energies deposited in the calorimeter to further discriminate electrons from photons. One of the main challenges for electron reconstruction comes from the events where one or more jets are misidentified as electrons.

The algorithm begins with initializing the EM clusters in the calorimeter through Simple Cone algorithm and CellNN algorithm [48]. The Simple Cone algorithm builds EM clusters from calorimeter towers within a cone of $\Delta R=\sqrt{\Delta \eta^{2}+\Delta \phi^{2}}=$ 0.4. The EM towers with the highest $E_{T}$ are chosen as the seeds and all the adjacent towers above the threshold $E_{T}=0.5 \mathrm{GeV}$ in the cone are added to the cluster. The CellNN algorithm chooses the calorimeter cell with the highest energy deposition as the seed, then adds neighbor cells to build the cluster. This is done at each calorimeter layer called floor clustering. The floor cluster from the third EM layer is 
chosen as the global cluster, to which the other floor clusters are matched with an angular requirement.

A set of criteria based on energy deposition, shower shape and track match are developed to identify electrons. The first is the electromagnetic fraction (EMF) $f_{E M}=\frac{E_{E M}(0.2)}{E_{\text {total }}(0.4)}>0.9$, where $E_{E M}(0.2)$ is the energy deposited within a cone of radius 0.2 in the EM calorimeter alone, while $E_{\text {total }}(0.4)$ is the energy deposited within a cone of radius 0.4 in both the EM calorimeter and hadronic calorimeter. The isolation fraction $f_{i s o}=\frac{E_{t o t a l}(0.4)-E_{E M}(0.2)}{E_{E M}(0.2)}$ is used to reject fake electrons with broader shower profiles by requiring $f_{\text {iso }}<0.15$.

Tracks in the central tracking system are searched and matched to the candidate electrons. A track match $\chi^{2}$ is defined as $\chi^{2}=\left(\frac{\Delta \phi}{\sigma_{\Delta \phi}}\right)^{2}+\left(\frac{\Delta z}{\sigma_{\Delta z}}\right)^{2}+\left(\frac{E_{T} / p_{T}-1}{\sigma_{E_{T}} / p_{T}}\right)^{2}$, where $\Delta \phi$ and $\Delta z$ are the differences on the azimuthal angle $\phi$ and $z$ position between the track and EM object projection at the $3^{\text {rd }}$ layer of EM calorimeter. The term $E_{T} / p_{T}$, the ratio of transverse calorimeter energy to the transverse momentum of the track, is approximately 1 in the case of electron. The track with the largest $\chi^{2}$ probability $P\left(\chi^{2}\right)$ is considered as the match to the electron candidate. The associated track and electron candidate should be within a cone of $\Delta \eta \times \Delta \phi=0.05 \times 0.05$.

More sophisticated variables, $\chi_{H M x 7}^{2}$ [49] and electron likelihood [50], are built to further select electrons. $\chi_{H M x}^{2}$ is a $\chi^{2}$ test on the longitudinal and transverse electron shower shapes using both data and simulated electrons, as $\chi^{2}=\sum_{i, j=1}^{7}\left(x_{i}^{k}-<\right.$ $\left.x_{i}>\right) H_{i j}\left(x_{j}^{k}-<x_{j}>\right)$, where $H_{i j}$ is the element of H-matrix. The H-matrix is the inverse of covariance matrix $M=\frac{1}{N} \sum_{n=1}^{N}\left(x_{i}^{n}-<x_{i}>\right)\left(x_{j}^{n}-<x_{j}>\right)$. The number $k$ and $n$ are the electron indices for data and simulated events. $x_{i}$ and $x_{j}$ are any of the seven shower shape variables including individual energy fractions in the 4 EM layers, $\log _{10}\left(E_{\text {total }}\right)$, primary vertex position $z_{p v} / \sigma_{z_{p v}}$ and shower width in $r-\phi$ plane 
of the $3^{\text {rd }}$ EM layer. An example of the $\chi^{2}$ distribution for real electrons and fake electrons is shown in Fig. 4.2(a) [14].

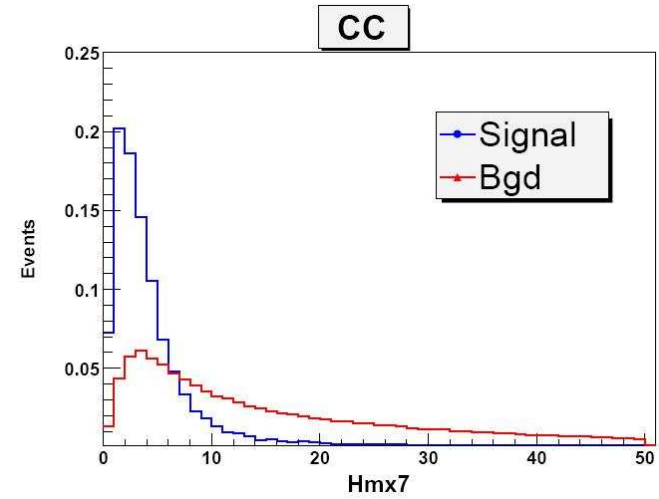

(a) $\chi^{2}$ test of $\operatorname{HMx} 7$

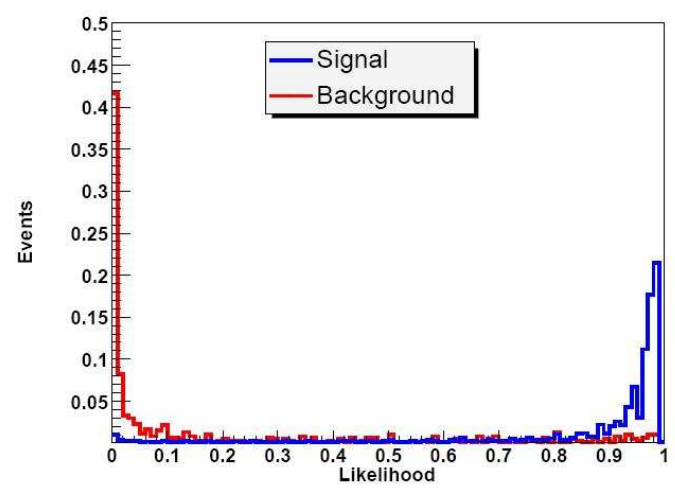

(b) electron likelihood

Figure 4.2: a) $\chi^{2}$ test on H-matrix with seven shower shape variables. b) Electron likelihood discriminant distribution. The real electron and fake electron events are both shown in the plots. [14]

The electron likelihood correlates the different parameters described above to effectively identify real electrons. The discriminant is defined as $L_{n}(x)=P_{\text {sig }}(x) /\left(P_{\text {sig }}(x)+\right.$ $\left.P_{b k g}(x)\right)$ with $P_{s i g}(x)=\prod_{i=1}^{7} P_{s i g, i}\left(x_{i}\right)$ and $P_{b k g}(x)=\prod_{i=1}^{7} P_{b k g, i}\left(x_{i}\right)$, where $x_{i}$ refer to the seven parameters: track match probability $P\left(\chi_{\text {spatial }}^{2}\right), E_{T} / p_{T}, \sum p_{T}$ of all tracks in cone $0.4, N_{\text {tracks }}$ in cone 0.05 around the cluster, $f_{E M}, d c a$ to the matched track to the primary vertex and $\chi_{H M x 7}^{2}$. The likelihood allows the variables to be weighted by their effectively in discriminating real electron from fake ones. The discriminant is shown in Fig. 4.2(b). The real electrons peaks around one while the fake events peaks at zero. A cut of likelihood $>0.85$ is often used to effectively reject the background especially due to the existence of neutral pions.

\subsubsection{Jet Reconstruction and Identification}

A jet is a narrow cone of energetic particles produced from the hadronization of partons or particle decays in hadron collisions. It leaves tracks in the central 
tracking system and deposits energy through hadronic shower in both the EM and hadronic calorimeters. Jets are reconstructed at D $\varnothing$ using two algorithms, the D $\varnothing$ RunII Cone algorithm and $k_{T}$ algorithm [15] [51], of which the former is used in this analysis with a cone size $\Delta R<0.5$. The algorithm in general is performed in three successive steps including clustering, addition of midpoints and merging/splitting.

It starts with clustering calorimeter cells into calorimeter towers which in turn are grouped to preclusters. Each calorimeter cell is considered massless object with a 4momentum vector $p_{\text {cell }}^{\mu}=\left(E_{\text {cell }}, \vec{p}_{\text {cell }}\right)$, where the momentum direction is determined by the primary vertex and the center of the cell. The noisy cells are discarded through NADA algorithm [52] [53] and the T42 algorithm [54] [55] [56]. The calorimeter cells in geometric range $\Delta \eta \times \Delta \phi=0.1 \times 0.1$ are built into calorimeter towers with corresponding 4-momentum vector $p_{\text {tower }}^{\mu}=\left(E_{\text {tower }}, \vec{p}_{\text {tower }}\right)=\sum_{\text {ith cell }}^{N \text { of cells }}\left(E_{i}, \vec{p}_{i}\right)$. The towers are ordered in descending $p_{T}$ and only those with $p_{T}>0.5 \mathrm{GeV}$ are considered as seeds for preclustering. If the most energetic cell in a tower is in $\mathrm{CH}$ layer or EC layers, this tower has to pass requirement $p_{T}^{\text {tower }}-p_{T}^{\text {the highest } p_{T} \text { cell }}>0.5$ $\mathrm{GeV}$. Starting with the highest $p_{T}$ tower as the seed, the towers within the cone size of 0.3 are moved into the precluster with its position weighted by tower energies. This procedure is repeated to form more preclusters in the rest towers exhaustively.

All of the preclusters are again sorted in descending $p_{T}$ order and those with $p_{T}<1 \mathrm{GeV}$ are discarded. The first precluster is used as the seed to build a protojet by adding up all the cells in cone $\Delta R=\sqrt{\Delta y^{2}+\Delta \phi^{2}}=0.5$. The rest preclusters are used to build proto-jets in the same way with proto-jet center axes separated by $\Delta R=0.25$ from each other. The proto-jet construction keeps going until $p_{T}^{\text {proto-jet }}<$ $4 \mathrm{GeV}$, or the axis of a proto-jet and its precluster differ by $\Delta R=0.001$, or 50 iterations. 
The seed-based clustering method is biased by the soft radiation of the jets. As illustrated in Fig. 4.3, if any of the two independent jets radiates a soft gluon, they might be reconstructed as a single jet through seed-based clustering. An additional list of proto-jets from clustering the midpoints of the previously formed proto-jet pairs is introduced to compensate this effect. The midpoint is considered if the pair of proto-jets satisfying $R_{\text {cone }}<\Delta R<2 R_{\text {cone }}$. Using these midpoints instead of preclusters, the similar clustering procedures are performed to build proto-jets without checking duplicates.
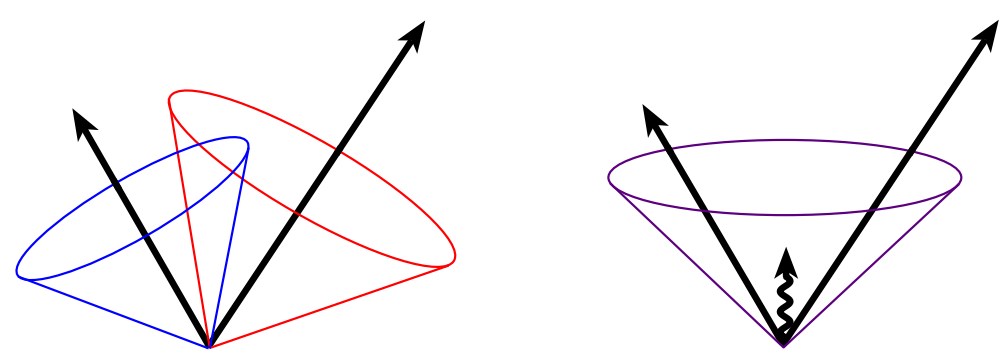

Figure 4.3: An illustration of the sensitivity in the seed-based cone jet clustering. In case there is soft gluon radiated by either of the two independent jets, the two jets could be treated as single jet in the seed-based cone jet clustering. [15]

The two lists of proto-jets are merged together by sorting $p_{T}$ in descending order. These proto-jets are merged and split into the final jets. If two proto-jets share calorimeter towers with energy larger than $50 \%$ energy of the lower $p_{T}$ proto-jet, the two proto-jets are merged into one. Otherwise they are split with the shared towers assigned to the closest proto-jet. The surviving proto-jets are computed and reconstructed into final jets.

These final jets are further selected with the following criteria to be used in the analysis: (1) To reject noise and EM objects, the EM fraction is required to be $5 \%<$ $E M F<95 \%$. (2) Because of the high noise in the Coarse Hadronic calorimeters, the fraction of energy deposited in these calorimeters has to satisfy $C H F<0.4$. (3) To remove fake jets from hot cells caused by electronics readout, the ratio of energy in 
the highest $p_{T}$ cell to that of the next highest $p_{T}$ cell in a jet, defined as HOTF, must be less than 10. (4) Similarly, $n 90$ is defined as the minimum number of calorimeter towers that together contain $90 \%$ energy of a jet. It is required that $n 90>1$. (5) The L1 trigger system is further used to confirm the jets. The confirmation is quantified as $L 1 C$ onf $=\left(\sum_{\text {trigger tower } i} E_{T}^{i}\right) /\left(E_{T}^{j e t}(1-C H F)\right)$, where L1Conf is required to be larger than 0.4 in the central and end calorimeters, and 0.2 in the intercryostat detector.

\subsubsection{Missing Transverse Energy}

Missing transverse energy $\left(\mathscr{H}_{T}\right)$ is considered as the experimental signature of neutrinos and other particles with negligible interactions when passing through the detector materials. The total transverse momentum in $p \bar{p}$ collisions is conserved to be zero in both initial and final states of the hard parton scattering. The calculation of $\mathbb{E}_{T}$ [57] [58] at D $\varnothing$ combines the energy deposition in the calorimeters, primary vertex in the central tracking system and energy correction in the muon detector. It is first determined as the negative vector sum of visible transverse energies of cells above $0.1 \mathrm{GeV}$ over all the calorimeters, as

$$
\overrightarrow{E_{T}}=-\sum_{i}{\overrightarrow{E_{T}}}^{i} \text {, with } E_{T}=\sqrt{\left|\overrightarrow{E_{T x}}\right|^{2}+\left|\overrightarrow{E_{T y}}\right|^{2}}
$$

Note that another useful variable called scalar $E_{T}$ is defined as the scalar sum of the transverse energies in all the calorimeter cells. The $\mathscr{H}_{T}$ is often corrected by muon momentum when a muon is present in an event and passes through the calorimeter losing a small fraction of its energy, typically $\sim 2 \mathrm{GeV}$. To be used in the analysis, the $\mathbb{E}_{T}$ must also be corrected by those corrections made to any physics objects like jets and electrons. 


\subsubsection{Muon Reconstruction and Identification}

Muons are reconstructed [59] [60] using hit information in the drift chambers and scintillators of the muon system, as well as tracks in the central tracking system. The hits in the A, B and C layers of the PDTs, MDTs and MSCs are first processed with position and time information. Using the default D $\varnothing$ linked list algorithm [61], these hits are linked and fit to local track segments, which in turn are filtered and transformed back to global track segments. The segments are paired in A layer and $\mathrm{BC}$ layers respectively and fit into local muon tracks with a fit algorithm. The momenta of the local tracks are determined by the curvature of the paths due to the toroidal magnetic field. The local muon tracks are finally matched to the global tracks in the central tracking system to improve the momentum resolution.

Muons from cosmic rays are rejected using the hit time information of scintillators and central track matching quality. As the muon candidate is associated with the bunch crossing, it is typically required to hit each layer within 10ns. The dca in transverse plane of the matched central track with respect to primary vertex in an event is required to be $\left|d c a_{T}\right|<0.2 \mathrm{~cm}$ without a SMT hit and $\left|d c a_{T}\right|<0.02 \mathrm{~cm}$ with SMT hits.

The reconstructed muon candidates are classified using the parameters of muon type and quality [17] [62]. The type of muon is described by nseg, of which $|n s e g|=1$, 2, or 3 means the muon has hits in only the A layer, only the B or $\mathrm{C}$ layer or all $\mathrm{A}, \mathrm{B}$ and $\mathrm{C}$ layers, respectively. A positive value of $n$ seg indicates that the muon candidate is matched to a central track, while a negative one indicates the muon can not be matched to a central track. The quality of a muon can be loose, medium or tight depending on number of hits in muon chambers and scintillators and the local muon fit. The combinations of type and quality are summarized in Tab. 4.1. In this 
analysis muons are selected to have $n s e g=+3$ medium, which requires hits in both

$\mathrm{A}$ and $\mathrm{BC}$ layers and a central track match.

\begin{tabular}{|c|c|c|}
\hline $\begin{array}{l}\text { muon type and } \\
\text { quality }\end{array}$ & hits in $\mathrm{A}$ and $\mathrm{BC}$ layers & tracks \\
\hline$|n s e g|=3$ tight & $\begin{array}{l}\text { at least two A layer wire hits } \\
\text { at least one A layer scintillator hit } \\
\text { at least three BC layer wire hits } \\
\text { at least one BC layer scintillator hit }\end{array}$ & $\begin{array}{l}\text { a converged local fit } \\
\chi_{\text {local }}^{2}>0\end{array}$ \\
\hline $\begin{array}{l}\mid \text { nseg } \mid=3 \\
\text { medium/loose }\end{array}$ & $\begin{array}{l}\text { medium: } \\
\text { at least two A layer wire hits } \\
\text { at least one A layer scintillator hit } \\
\text { at least two BC layer wire hits } \\
\text { at least one BC scintillator hit (except for central muons } \\
\text { with less than four BC wire hits) } \\
\text { loose: } \\
\text { allow one of the above tests to fail, with the A wire and } \\
\text { scintillator requirement treated as one test and requiring } \\
\text { always at least one scintillator. }\end{array}$ & $\begin{array}{l}\mathrm{A} \text { and a BC seg- } \\
\text { ments matched or } \\
\text { not with a central } \\
\text { track. }\end{array}$ \\
\hline $\begin{array}{ll}\text { nseg } \quad= & +2 \\
\text { loose/medium } & \end{array}$ & $\begin{array}{l}\text { loose: } \\
\text { at least one BC scintillator hit } \\
\text { at least two BC layer wire hits } \\
\text { medium: } \\
\text { fulfills the above requirements and if it is located in the } \\
\text { bottom part of the detector (octant } 5 \text { and } 6 \text { with }\left|\eta_{D}\right|<1.6 \text { ) }\end{array}$ & $\begin{array}{l}\text { BC segments } \\
\text { matched with a } \\
\text { central track. }\end{array}$ \\
\hline $\begin{array}{ll}\text { nseg }= & +1 \\
\text { loose/medium } & \end{array}$ & $\begin{array}{l}\text { loose: } \\
\text { at least one A layer scintillator hit } \\
\text { at least two A layer wire hits } \\
\text { medium: } \\
\text { fulfills the above requirements and if it is located in the } \\
\text { bottom part of the detector (octant } 5 \text { and } 6 \text { with }\left|\eta_{D}\right|<1.6 \text { ) } \\
\text { Low momentum nseg }=1 \text { muons are also defined as medium. } \\
\text { A nseg }=1 \text { muon is qualified as a low momentum muon if its } \\
\text { probability to reach the BC layer is less than a parameter } \\
0.7 \text { set by RCP. }\end{array}$ & $\begin{array}{l}\text { A segment matched } \\
\text { with a central track }\end{array}$ \\
\hline
\end{tabular}

Table 4.1: Overview of the definitions of muon type and quality. [17]

In order to further control the muon purity, the matched central track is certified with 3 quality levels, loose, medium and tight, using SMT hits, track fit $\chi^{2}$ and $d c a$ to primary vertex. A track is defined as loose if $\left|d c a_{T}\right|<0.2 \mathrm{~cm}$ if there is no SMT hit and tightens to $0.02 \mathrm{~cm}$ in case it has SMT hits. A track is medium if it passes the loose requirement and the track fit satisfies $\chi^{2} /$ d.o.f $<4$. A tight track fulfills the medium requirement and has SMT hits. The medium quality track is used in 
the analysis.

The calorimeter information is also used to discriminate the muons in leptonic decay from those muons in heavy flavor hadron decay. Muons originating from a leptonic decay of $W$ boson $(W \rightarrow \mu \nu)$ tend to have a relatively large transverse momentum and be isolated from reconstructed jets, while muons from semileptonic decay of heavy flavor hadrons $(B \rightarrow \mu \nu X)$ appear to be less energetic and inside a jet. Thus a muon is often required to be separated from the nearest reconstructed jet having $p_{T}>6 \mathrm{GeV}$ by $\Delta R(\mu$, jet $)>0.5$. In addition, two variables based on the energy clustered in the cones around muons are used to determine the isolation of muons, as

$$
\begin{gathered}
\text { ScaledCalorimeter Halo }=\frac{\sum_{\text {cell } i} E_{T}^{i}}{p_{T_{\text {muon }}}}, 0.1<\Delta R(\text { calo cells }, \text { muon })<0.4 \\
\text { ScaledTrackHalo }=\frac{\sum_{\text {track } k} p_{T}^{k}}{p_{T_{\text {muon }}}}, \Delta R(\text { tracks }, \text { muon })<0.5
\end{gathered}
$$

where the calorimeter cell energies are summed in a hallow cone between 0.1 and 0.4 and the momenta of tracks are summed in cone 0.5 with muon momentum subtracted. In this analysis, the isolation variables are required to satisfy ScaledCalorimeter Halo < 0.08 and ScaledTrackHalo $<0.06$.

\subsection{Corrections to Physics Objects}

\subsubsection{Jet Energy Scale, JetSSR and Jet ID Efficiency Corrections Jet Energy Scale}

The Jet Energy Scale (JES) correction is used to correct the measured jet energy in the detector back to the total energy of all the final state particles inside a jet cone [63] [64]. The correction is in general expressed as

$$
E_{\text {jet }}^{\text {corr }}=\frac{E_{\text {jet }}^{\text {meas }}-E_{\text {offset }}}{k_{R} R \times S} k_{\text {offset }}
$$


where $E_{\text {jet }}^{\text {corr }}$ is the corrected jet energy, $E_{\text {jet }}^{\text {meas }}$ is the measured jet energy, $E_{\text {off set }}$ is the offset energy, $R$ is the calorimeter response, $S$ is showering correction, $k_{R}$ and $k_{\text {offset }}$ are correction factors for the JES determination process itself.

The offset energy $E_{\text {offset }}$ is the excessive energy inside a jet cone due to the noise from electronics and the uranium absorber's radioactive decay, additional $p \bar{p}$ interactions and previous bunch crossing energy pileup. The average offset energy is estimated from the energy deposition in minimum bias and zero bias data events. Because of the overestimation, it is corrected again using $\gamma+j e t$ simulated events. As shown in Fig. 4.4, the offset energy is typically a few GeV and increases with jet detector peseudorapidity, the number of primary vertices, and instantaneou luminosity.

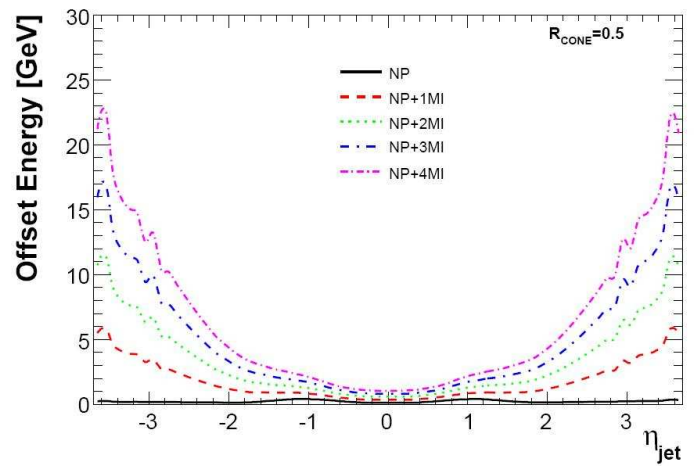

(a) E offset Run IIa

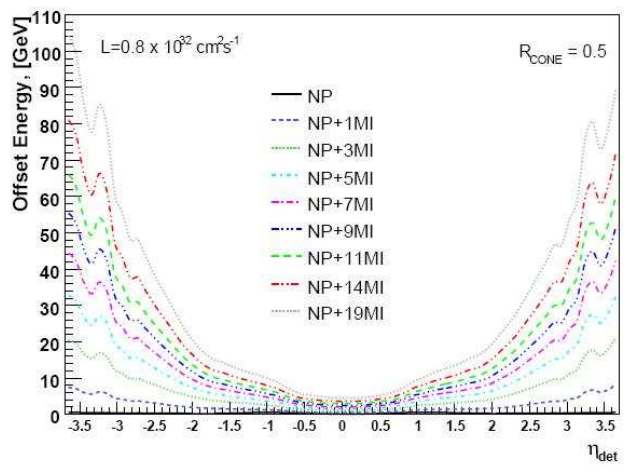

(b) $E_{\text {offset }}$ Run IIb

Figure 4.4: Offset energy as a function of jet detector eta $\eta_{\text {det }}$. a) Energy offset determined in Run IIa datasets. b) Energy offset determined in Run IIb dataset. Its dependence on number of primary vertices is also shown in the plots.

$R$ is the detector response of the calorimeter to particle jets. It is usually less than 1 because of dead materials, poorly instrumented regions, lower response of hadrons compared to EM objects, and the inhomogeneous detector layout. The average response is measured using $X+$ jet samples, where $X$ could be $\gamma, Z$ or jet. A so-called Missing $E_{T}$ Projection Fraction (MPF) method is developed to measure 
the response to data events. In this method, the well measured EM energy scale is used to compute the response to hadrons. As shown in Fig. 4.5, this correction is dependent on the jet detector pseudorapidity and jet energies. It is the largest relative correction (up to $30 \%$ of the jet energy).

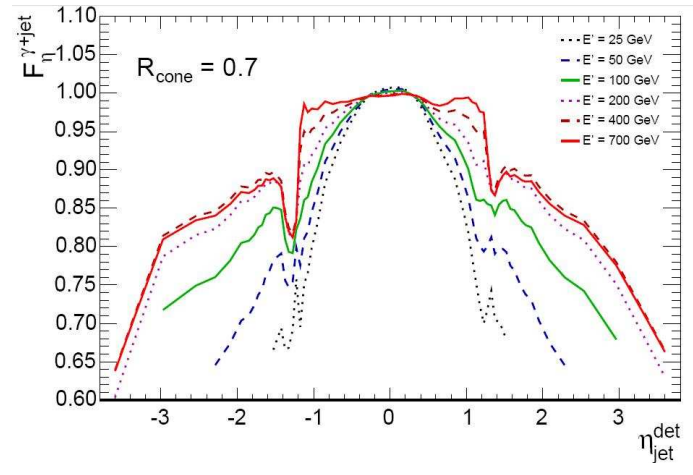

(a) Relative MPF correction in Run IIa

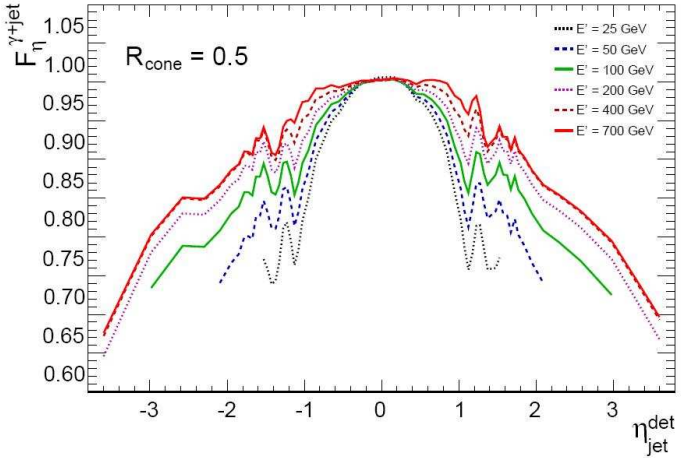

(b) Relative MPF correction Run IIb

Figure 4.5: Relative MPF Correction using $\gamma+j e t$ in data events a function of jet detector eta $\eta_{\text {det }}$. a) with 0.7 jet cone Run IIa datasets. b) With 0.5 jet cone in Run IIb datasets. Its dependence on the energy is also shown in the plots.

The showering correction is used to compensate for the energy flow outside of the jet cone. During the shower development, particles could escape out of the jet cone due to interactions with the materials, bending in the magnetic fields and limited jet cone size. Its average correction is determined using $\gamma+$ jet events for both data and simulated events by measuring the shower profiles. As shown in Fig. 4.6, it is parametrized as a function of jet transverse energy. The showering correction is relatively small ( $\sim 5 \%$ in the small $\left|\eta_{\text {det }}\right|$ region).

The total JES correction is parameterized as a function of jet peseudorapidity as well as measured jet energy. Fig. 4.7 is an example for Run IIb jet with a cone size of 0.5 determined using $\gamma+$ jet events. In the central detector region, the scales are almost flat and varies from $15 \%$ to $45 \%$ depending on the measured jet transverse energies. Note that the heavy flavor jets which decay to muons are also corrected 


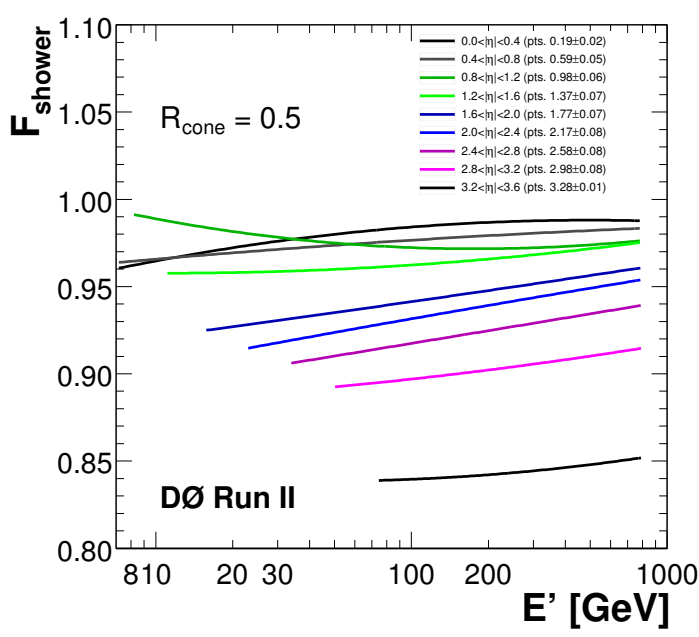

(a) Showering correction in jet cone 0.5

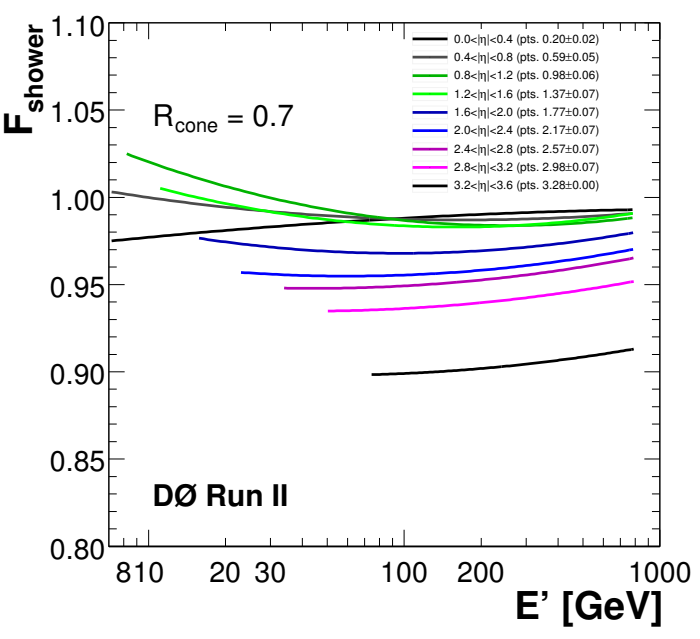

(b) Showering correction in jet cone 0.7

Figure 4.6: Showering correction using $\gamma+$ jet events as a function of jet energy in Run IIa. a) With 0.5 jet cone. b) With 0.7 jet cone. Its dependence on detector eta is also shown in the plots.

by approximately twice the muon energies. The total systematic uncertainty of JES correction is in general less than $5 \%$, and close to $1 \%$ in the central detector region for RunIIb datasets.

\section{JetSSR}

Due to limitations in D $\varnothing$ detector modeling, the reconstructed and JES corrected jets of MC simulated events have different reconstruction efficiency, energy scale and resolution compared to the jets in data events. A method called Jet Smearing, Shifting and Removing (JetSSR) [65] [66] is designed to cope with these differences.

The $\gamma+j e t$ events from both data and simulated event samples are selected with the photon and jet back-to-back in $\phi$. The difference between data and simulated events are quantified as a function of photon transverse momentum $p_{T}^{\gamma}$

$$
\mathcal{D}=<\Delta S>_{\text {data }}-<\Delta S>_{M C}
$$




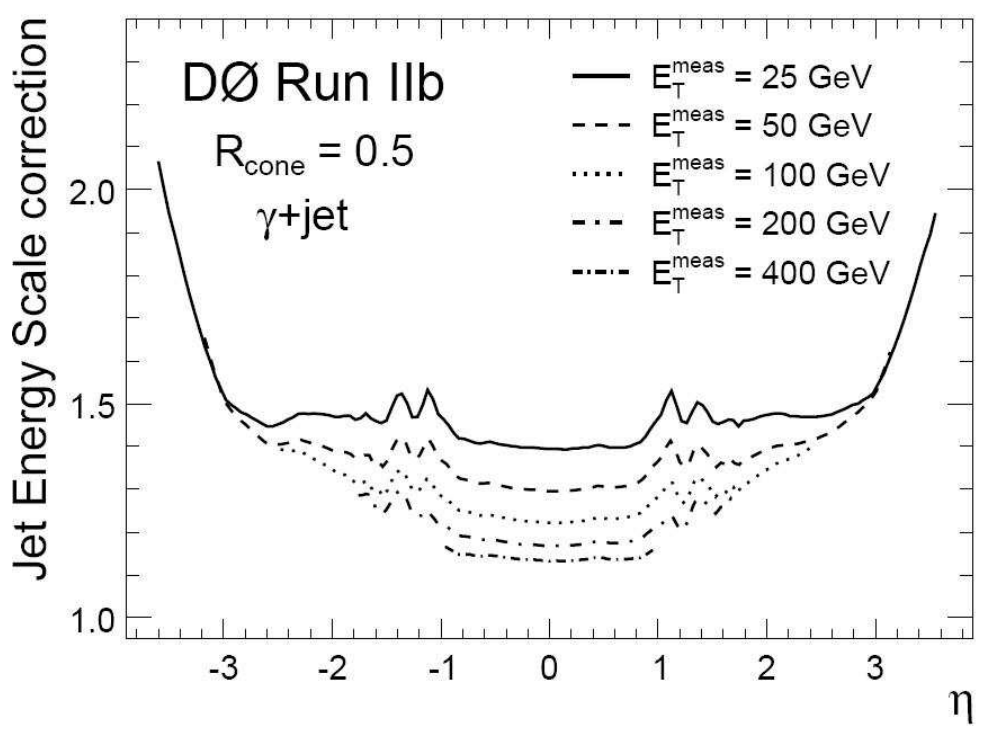

Figure 4.7: The total JES correction as a function of jet pseudorapidity using $\gamma+j e t$ events in Run IIb datasets. Different curves correspond to different measured jet transverse energies.

where $\Delta S=\left(p_{T}^{j e t}-p_{T}^{\gamma}\right) / p_{T}^{\gamma}$ is calculated in each bin of the $p_{T}^{\gamma}$ spectrum for data and simulated events, respectively. To extract the difference from $\mathcal{D}\left(p_{T}^{\gamma}\right)$, a Gaussian function multiplied with an error function is used to deconvolute the information. The jet energy scale and resolution differences between data and $\mathrm{MC}$ events are the mean value and width of the Gaussian function respectively, while the jet identification inconsistency is obtained from the turn-on curve, which is described by error functions.

The resulting corrections are then applied on simulated events. First of all, the transverse energy resolution correction factor, as shown in Fig. 4.8, is applied by smearing jet $p_{T}$ with a Gaussian function of width $\sigma_{\text {smear }}=\sqrt{\sigma_{\Delta S_{\text {data }}}^{2}-\sigma_{\Delta S_{M C}}^{2}}$. The jet energies are shifted by the JES correction difference, of which an example in central detector region is shown in Fig. 4.9. As to the jet identification efficiency difference, as shown in Fig. 4.10, the jet $p_{T}$ turn-on curves of both data and simulated events reach their plateau at $p_{T}>15 \mathrm{GeV}$. Thus a simple cut of $15 \mathrm{GeV}$ is applied to remove the data and simulated events with $p_{T}<15 \mathrm{GeV}$. 


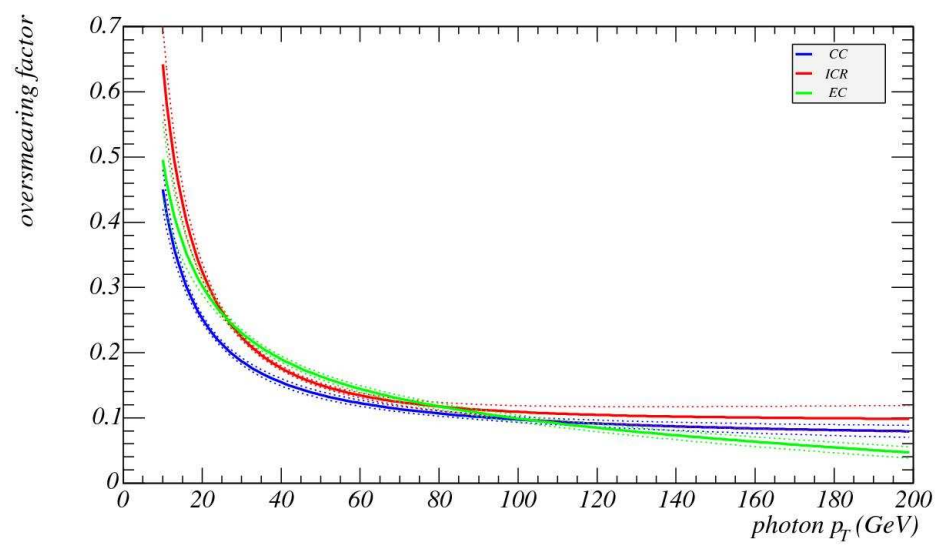

Figure 4.8: The smearing factor as a function of $p_{T}^{\gamma}$ applied to simulated events. The curves represent different detector regions: CC (blue), ICR (red) and EC (green). The dashed curves reflect the statistical uncertainties only.

\section{Jet ID Efficiency Correction}

The jet identification efficiency [16] [67] refers to the efficiency of jets passing the basic selection criteria such as EMF, CHF and L1Conf described in Section 4.1.3. It is determined using the $\gamma+j e t$ and dijet event samples via the tag-and-probe method. The efficiency is extracted for each basic cut and combined into a single efficiency for data and simulated events separately. The difference between the efficiencies of data and simulated events are parameterized as functions of $p_{T}$ and $\eta_{D}$. As shown in Fig. 4.11, the scale factor as a function of $\eta_{D}$ for the jet in the identification plateau region is close to 1 at $\sim 2 \%$ level. The scale factor is then applied to simulated event samples by rejecting simulated events with a probability of $1-S F$ in each $p_{T}$ and $\eta_{D}$ bin.

\subsubsection{Lepton ID Efficiency Correction}

The transverse momenta of simulated electrons and muons are smeared in ways similar to the jet smearing to mimic the momentum resolution of data events. For instance, the muon smearing parameter is determined by fitting the $Z$ peak of the 


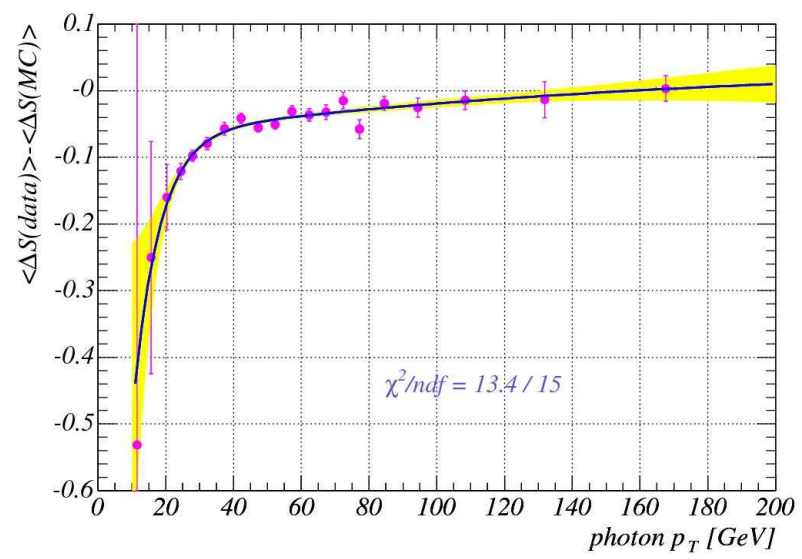

Figure 4.9: The difference in jet energy scale correction between data and simulated events, as a function of $p_{T}^{\gamma}$. Shown in the plot is for CC detector region only. The yellow band represents the statistical errors.

$\mu^{+} \mu^{-}$invariant mass in the $Z \rightarrow \mu^{+} \mu^{-}$distribution using a Gaussian function convoluted with the Drell-Yan spectrum. The smearing parameters as a function of muon $p_{T}$ for Run IIa datasets are shown in Fig. 4.12.

The difference in the lepton identification efficiencies between data and simulated events are also estimated with different lepton qualities. The muon identification efficiency is determined using $Z \rightarrow \mu^{+} \mu^{-}$event samples through the tag-and-probe method. The scale factors for the differences between data and simulated events are calculated for the corresponding muon qualities. In this analysis, the muon quality used is medium nseg $=3$ and the scale factor is parameterized in $\phi$ and $\eta$. As shown in Fig. 4.13, the scale factor as a function of $\eta$ for medium nseg $=3$ muon is on average $\sim 0.97 \pm 0.007$. Similarly, a tracking efficiency correction is applied for the corresponding track quality and parameterized as functions of $z$ and $\eta_{C F T}$. The medium track is used in this analysis, as shown in Fig. 4.14. The average scale factor is $\sim 0.93 \pm 0.021$, with the beam systematic already included. The muon isolation cuts used in the analysis are $\Delta R(\mu$, jet $)>0.5$ and TopP14Tight as described in Section 4.1.5. The corresponding scale factors are parameterized as functions of 


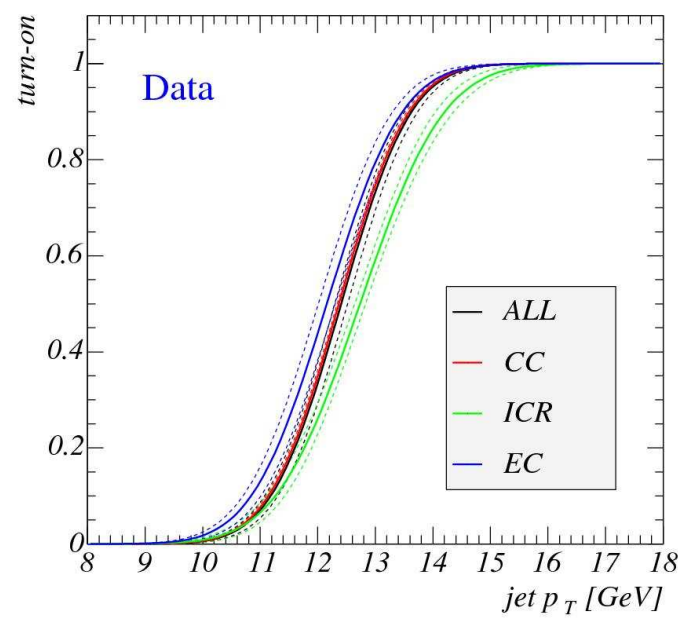

(a) Data

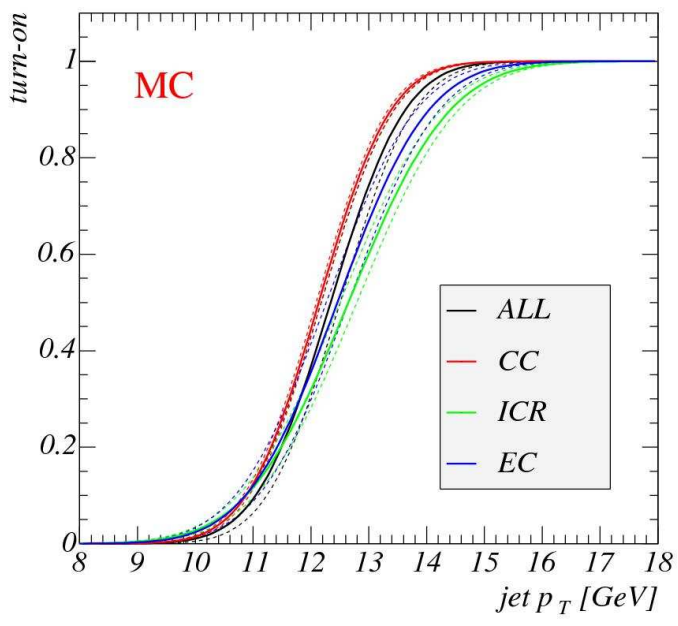

(b) MC

Figure 4.10: The turn-on curves of jet identification efficiencies as a function of jet $p_{T}$. a) Data. b) MC. The dashed curves represent statistical errors only.

muon $p_{T}$ and the number of jets with $p_{T}>15 \mathrm{GeV}$, as shown in Fig. 4.15 and 4.16 .

\subsubsection{Missing $E_{T}$ Recalculation}

As discussed in Section 4.1.4, the missing $E_{T}$ is the negative vector sum of all calorimeter cell energy except for the unclustered energy in the coarse hadronic calorimeter due to its poor resolution [68]. The transverse energy of the muons is also subtracted. All the corrections to jets, muons and electrons are propagated into the final calculation of missing $E_{T}$. Note that in the Run IIb analysis, JetSSR correction is excluded from the recalculation of missing $E_{T}$ to minimize the effects from irrelevant energies out of the jet cones.

\subsection{Identification of $\mathrm{b}$ Jets}

\subsubsection{The DØ Neural Network b-jet Tagger}

The average lifetime of b hadrons is $1.564 \pm 0.014 p s$ [69], much longer compared to those of light flavored hadrons. The $\mathrm{b}$ hadrons produced in $p \bar{p}$ collisions typically 


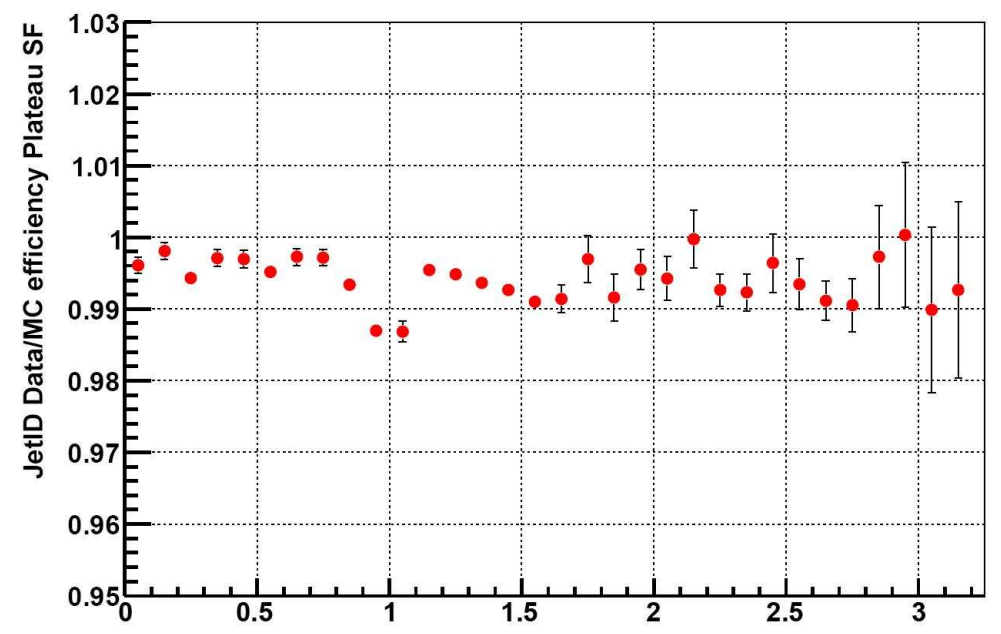

Figure 4.11: The scale factor for correcting jet identification efficiency difference between data and simulated events, as a function of $\left|\eta_{D}\right|$ binned by 0.1 . The scale factor is determined using the jets in the reconstruction efficiency plateau region and Run IIb datasets only.[16]

travel a measurable distance of several $\mathrm{mm}$ and decay to other charged particles, from which a secondary vertex can be reconstructed [70]. Most of the decayed particles from heavy b hadrons are inside a cone of $\Delta R=0.5$ around the $\mathrm{b}$ jet axis but carry significant momenta in the transverse direction relative to the jet axis. The reconstructed tracks hence are separated from the primary vertex by corresponding impact parameters, defined as the distance of the closest approach of the track to the primary vertex, as shown in Fig. 4.17. Based on the above characteristics of b jets, the DØ b-tagging algorithms are developed to discriminate b jets from light jets.

\section{SVT Tagger}

The Secondary Vertex (SVT) Tagger [71] identifies a b jet by fitting the secondary vertex associated with the jet. Other long lived particles such as $\Lambda$ and $K_{s}^{0}$ are excluded using the invariant mass information of the two-track vertex. At least two tracks with impact parameter significance $I P / \sigma_{I P}>3$ and at least $7 \mathrm{CFT}$ hits are used to fit a secondary vertex, of which the decay length is further required to satisfy $\left|\overrightarrow{L_{x y}}\right| / \sigma_{\left|\overrightarrow{L_{x y}}\right|}>5$. The calorimeter jet within $\Delta R<5$ is matched to the secondary 


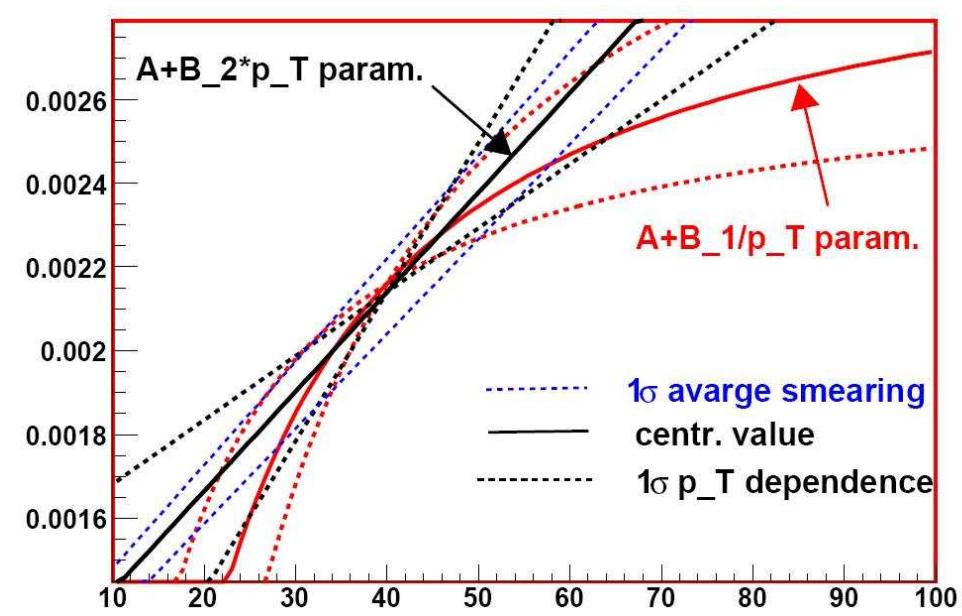

Figure 4.12: The muon smearing parameters as a function of muon $p_{T}$ for events where both muons have SMT hits and $\left|\eta_{C F T}\right|<1.6$. The dashed lines represent the $1 \sigma$ uncertainties of the two parameterizations. [17]

vertex and identified as $\mathrm{b}$ jet. The average b-tagging efficiency is $38.9 \%$ with a fake rate of $\sim 0.47 \%$.

\section{CSIP Tagger}

The Counting Signed Impact Parameter (CSIP) Tagger [72] combines the number of tracks and their impact parameter significances to tag the jets. As shown in Fig. 4.17, the IP is called positive if the track intersects with the jet axis in front of the primary vertex. Otherwise the IP is negative if the track intersects with jet axis behind the primary vertex. Jets containing at least 2 tracks with $I P / \sigma_{I P}>3$ or at least 3 tracks with $I P / \sigma_{I P}>2$ are considered to be $\mathrm{b}$ jets. The average b-tagging efficiency on data is $(23 \pm 1) \%$ with a fake rate of $\sim 2 \%$.

\section{JLIP Tagger}

The Jet Lifetime Probability (JLIP) Tagger [73] uses the impact parameters of tracks in a jet to build a probability $P_{j e t}$, that all the tracks in the jet come from the 


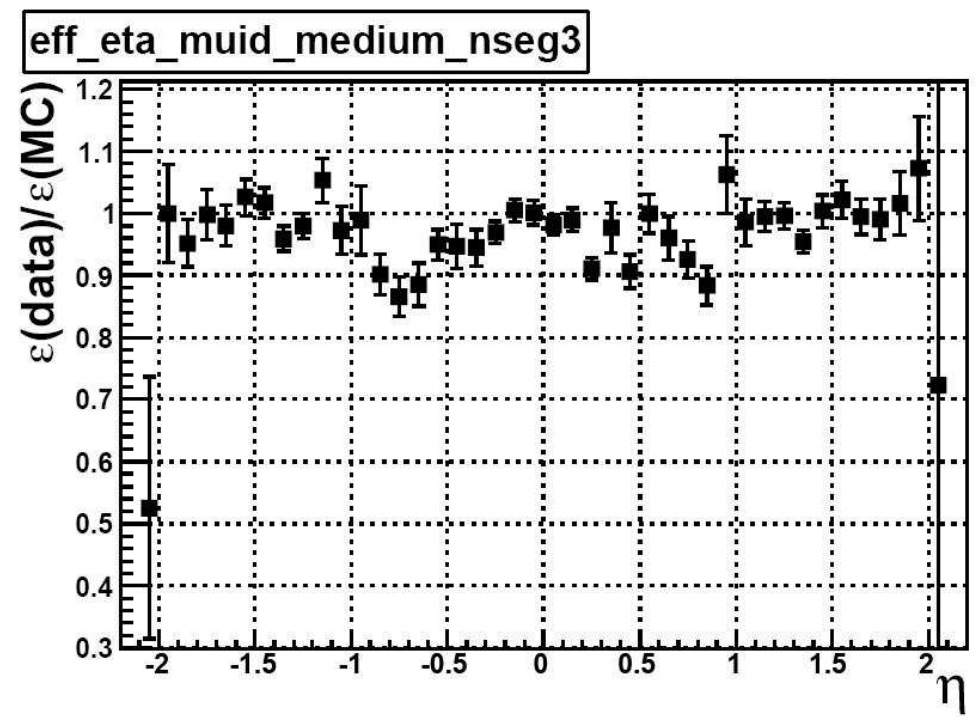

Figure 4.13: The correction factor of medium nseg $=3$ muon identification efficiency as a function of $\eta$. Muons in the hole regions are not considered.

primary vertex, as

$$
P_{j e t}=\Pi \cdot \sum_{i=0}^{N_{t r k}-1} \frac{(-\ln \Pi)^{i}}{i !}
$$

where $N_{t r k}$ is the number of tracks and $\Pi$ is the product of the probabilities $P_{t r k}$ for all tracks of the jet, which represent the probability of a track to originate from the primary vertex, as

$$
P_{t r k}=\frac{\int_{s_{0}}^{\infty} R(s) d s}{\int_{0}^{\infty} R(s) d s}
$$

The resolution function $R(s)$ is determined by fitting the negative impact parameter distributions using a sum of four Gaussian functions. The values of $P_{j e t}$ for light jets are uniform between 0 and 1 , while those for $\mathrm{b} / \mathrm{c}$ jets peak at small values close to 0 . A set of 6 working points corresponding to cutoff values ranging from 0.001 to 0.04 is used to select b jets. The b-tagging efficiency with a cutoff of 0.02 can reach up to $\sim 60 \%$ with a fake rate around $2 \%$. 


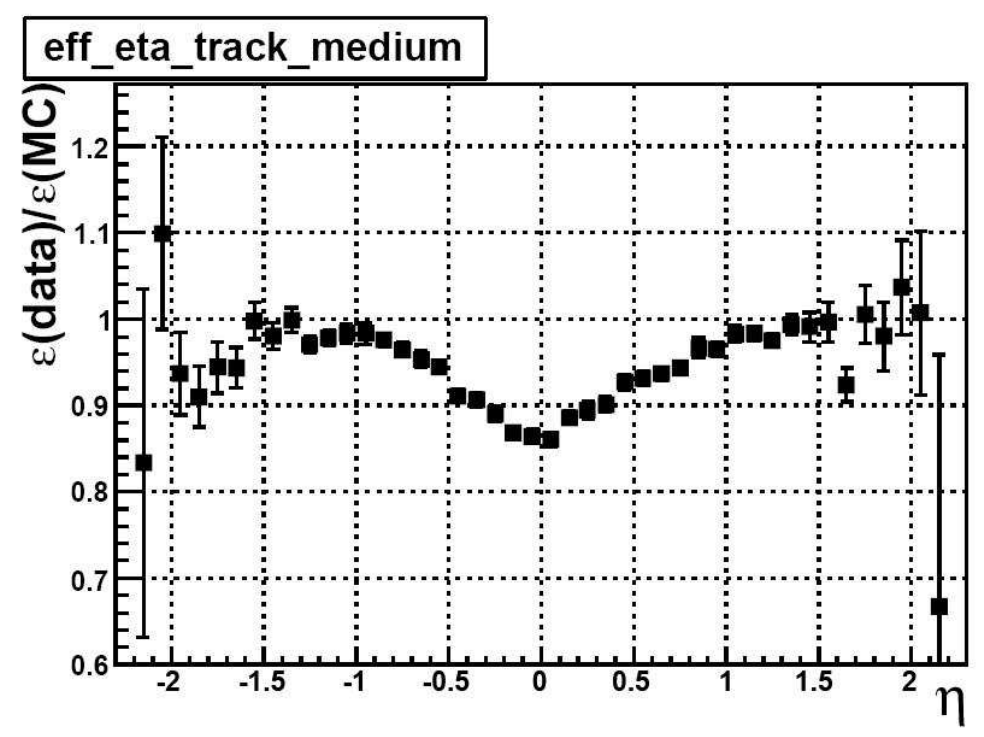

Figure 4.14: The correction factor of medium tracking efficiency as a function of CFT detector $\eta$.

\section{Soft Lepton Tagger}

The Soft Lepton Tagger (SLT) [74] identifies b jets by looking for energetic muons inside the jets. B hadrons decay into muons via $b \rightarrow \mu X$ and $b \rightarrow c \rightarrow \mu X$ with total branching ratio of $\sim 20 \%$. The resulting muons have relatively large momenta $p_{T}^{\text {rel }}$ in the transverse direction with respect to the jet axis. Since the soft muon tagging is independent of those algorithms based on b hadron lifetime, it is often used to determine the b-tagging efficiency of the Neural Network b-jet tagger by fitting $p_{T}^{\text {rel }}$ templates.

\section{DØ Neural Network b-jet Tagger}

The DØ Neural Network (NN) b-jet tagger [18] [75] correlates the SVT, CSIP and JLIP algorithms as well as 4 additional relevant variables to discriminate b jets from light jets. The seven input variables are chosen for their b-jet discrimination power, as shown in Tab. 4.3. Five of them are based on the SVT, while the other two are based on the CSIP and JLIP algorithms. The optimized NN parameters are 


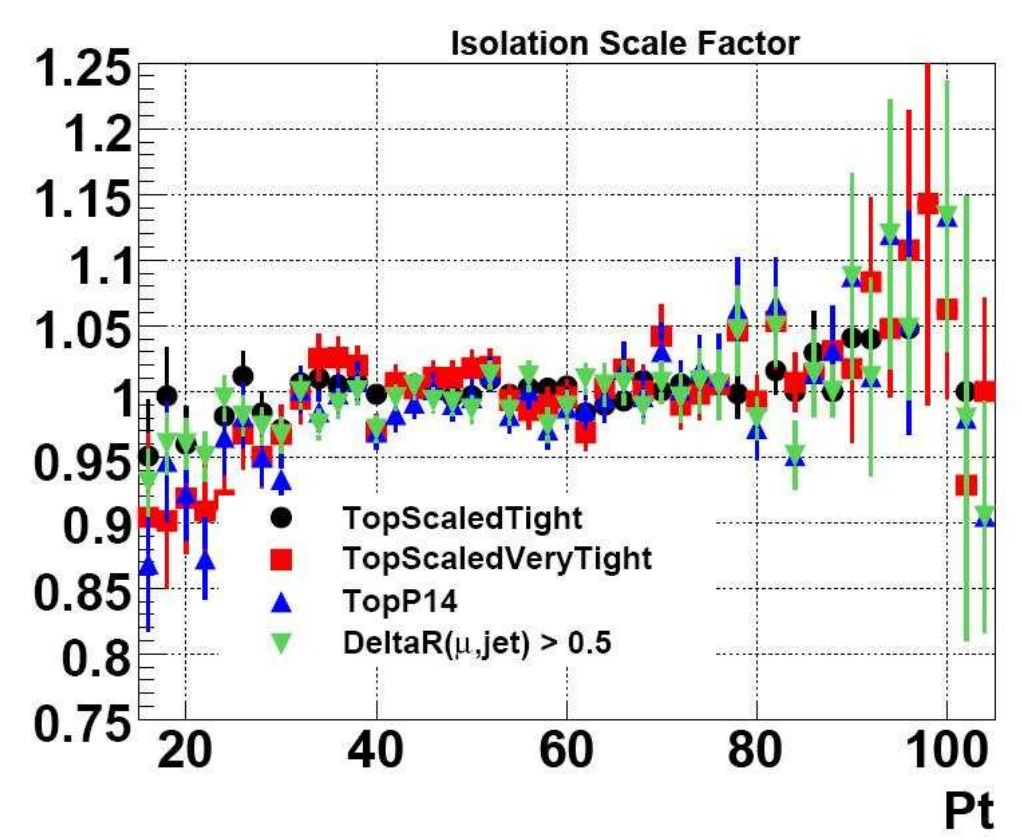

Figure 4.15: The correction factors of muon isolation efficiencies as a function of muon $p_{T}$.

shown in Tab. 4.2. The seven input nodes and one output node are corresponding to the seven input variables and one NN output. The number of hidden nodes are optimized to be 24 after varying number of hidden nodes from 7 to 24 . The training of the neural network is performed on $b \bar{b}$ and $q \bar{q}$ samples with 400 epochs.

\begin{tabular}{|l|l|}
\hline Parameter & Value \\
\hline NN Structure & $7: 24: 1: 1$ \\
\hline $\begin{array}{l}\text { Jet input selection cuts (failure } \\
\text { results in NN output of 0) }\end{array}$ & $\begin{array}{l}S V T_{S L} \text { DLS }>2.5 \text { or } \\
\text { JLIP Prob < 0.02 or } \\
C S I P \text { Comb }>8\end{array}$ \\
\hline Number of training epochs & 400 \\
\hline $\begin{array}{l}\text { Number of b jets used in training } \\
\text { (after jet selection cuts) }\end{array}$ & $389,109(312,549)$ \\
\hline $\begin{array}{l}\text { Number of fake jets used in train- } \\
\text { ing (after jet selection cuts) }\end{array}$ & $1,672,879(331,751)$ \\
\hline
\end{tabular}

Table 4.2: NN parameters. [18]

The NN output is a continuous distribution between 0 and 1 , where light jets appear to have small values around 0 and $\mathrm{b}$ jets peak at 1 . A set of 12 operation points are evaluated to classify the performance of the NN tagger. As shown in Tab. 4.4, each operating point corresponds to a cutoff value to identify b jets. Their b- 


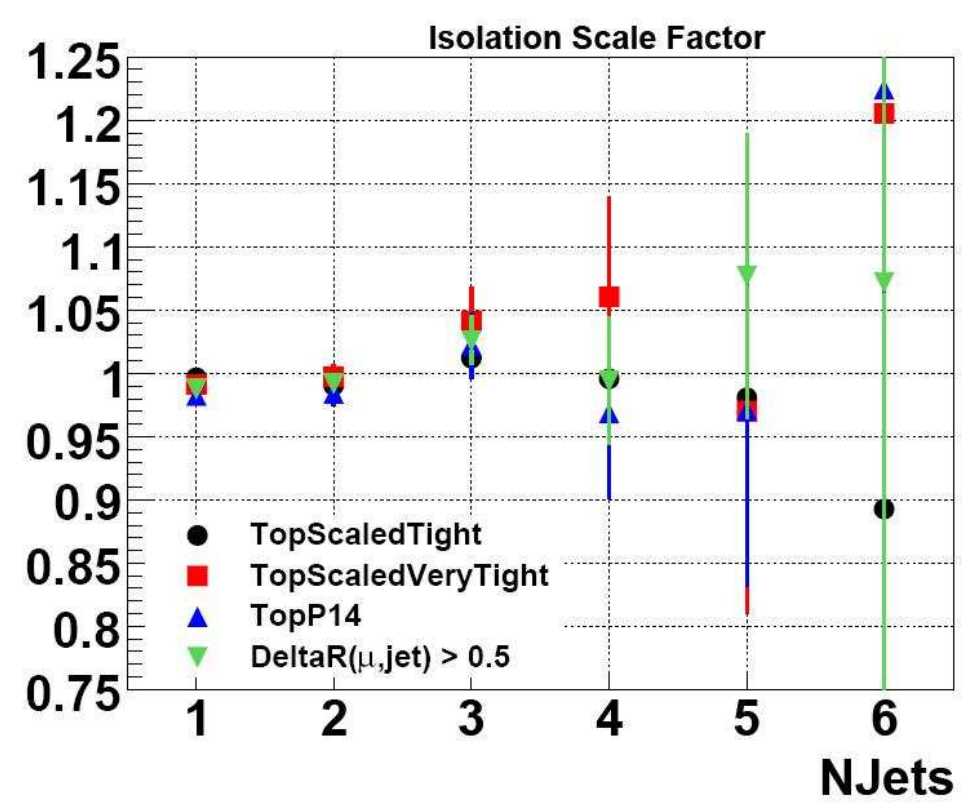

Figure 4.16: The correction factors of muon isolation efficiencies as a function of number of jets with $p_{T}>15 \mathrm{GeV}$.

tagging efficiencies on data are measured using the System8 formalism [76], of which the solutions to the 8 equations include the efficiencies of SLT and NN taggers. The data performance is evaluated using $Z \rightarrow b \bar{b}$ and $Z \rightarrow q \bar{q}$ samples. As shown in Fig. 4.18 , the performance is parametrized into $2 \mathrm{D}$ functions with fake rates ranging from $0.2 \%$ to $11 \%$.

\begin{tabular}{|l|l|l|}
\hline Rank & Variable & Description \\
\hline 1 & $S V T_{S L}$ DLS & $\begin{array}{l}\text { Decay length significance of the sec- } \\
\text { ondary vertex }\end{array}$ \\
\hline 2 & CSIP Comb & $\begin{array}{l}\text { Weighted combination of the tracks' IP } \\
\text { significance }\end{array}$ \\
\hline 3 & $J L I P$ Prob & $\begin{array}{l}\text { Probability that the jet originates from } \\
\text { the primary vertex }\end{array}$ \\
\hline 4 & $S V T_{S L} \chi_{\text {dof }}^{2}$ & $\begin{array}{l}\text { Chi square per degree of freedom of the } \\
\text { secondary vertex }\end{array}$ \\
\hline 5 & $S V T_{L} N_{\text {Track }}$ & $\begin{array}{l}\text { Number of tracks used to reconstruct } \\
\text { the secondary vertex }\end{array}$ \\
\hline 6 & $S V T_{S L}$ Mass & Mass of the secondary vertex \\
\hline 7 & $S V T_{S L}$ Num & $\begin{array}{l}\text { Number of secondary vertex found in } \\
\text { the jet }\end{array}$ \\
\hline
\end{tabular}

Table 4.3: The Neural Network input variables ranked in order of b-jet discrimination power. [18] 


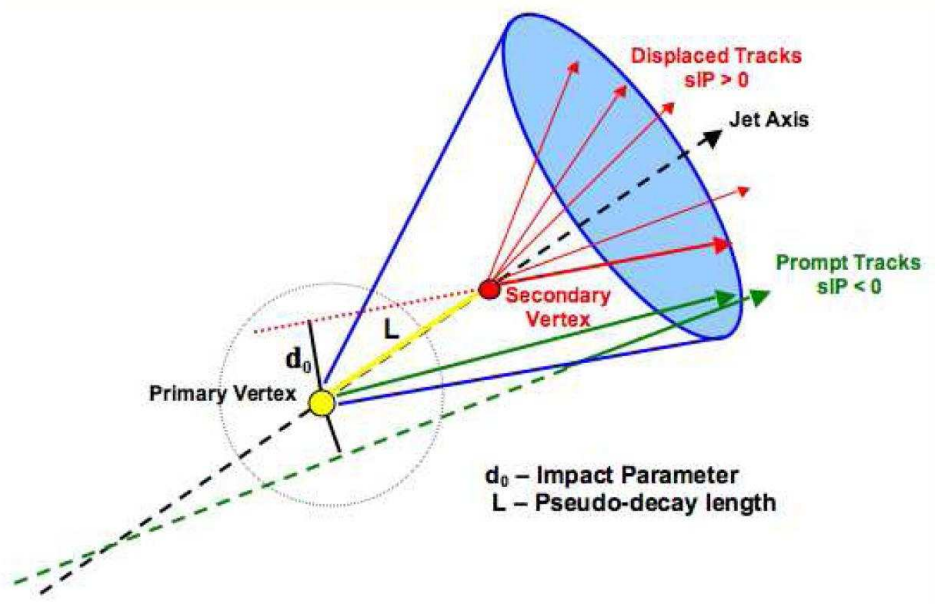

Figure 4.17: A schematic view of b hadron production and decay in $p \bar{p}$ collisions. $L$ is the b hadron decay length, while $d_{0}$ is the impact parameter for one of the tracks.

\begin{tabular}{|c|c|c|c|c|c|c|}
\hline Name & MegaTight & UltraTight & VeryTight & Tight & Medium & oldLoose \\
\hline NN Cut & $>0.925$ & $>0.9$ & $>0.85$ & $>0.775$ & $>0.65$ & $>0.5$ \\
\hline Name & Loose & L2 & L3 & L4 & L5 & L6 \\
\hline NN Cut & $>0.45$ & $>0.325$ & 0.25 & $>0.2$ & $>0.15$ & $>0.1$ \\
\hline
\end{tabular}

Table 4.4: The operation points of the DØ NN b-jet tagger. [18]

\subsubsection{Tag Rate Function and Taggability}

The Tag Rate Functions (TRFs), namely TRFb, TRFc and TRFl, are introduced to describe the probabilities that the b, $\mathrm{c}$ and light jets in the event are tagged respectively. They are determined at the same time when measuring the NN tagger performance on data using the System8 method which includes the b jet tagging efficiencies in the equations. Because the simulated events overestimate tracking quality compared to data samples, their b-tagging efficiencies are overestimated by up to $\sim 20 \%$. To take this difference into account, the scale factors are measured using $b \rightarrow \mu$ samples and applied to all simulated samples, as

$$
S F_{b \rightarrow \mu}=\frac{\epsilon_{b \rightarrow \mu}^{d a t a}}{\epsilon_{b \rightarrow \mu}^{M C}}
$$

and

$$
T R F b=\epsilon_{b}^{M C} \times S F_{b \rightarrow \mu} .
$$




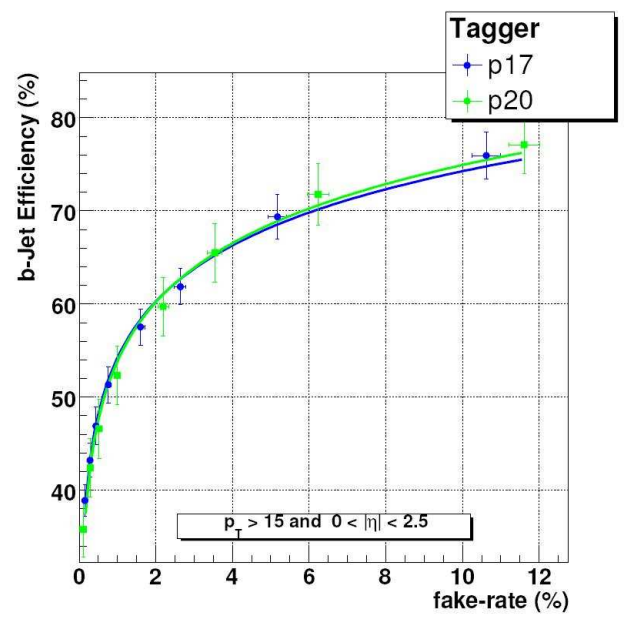

Figure 4.18: The b-jet tagging efficiency versus the tagging fate rate for the 12 operation points of the $\mathrm{D} \varnothing \mathrm{NN}$ b-jet tagger. The blue dots are determined on Run IIb datasets while the red dots are determined on Run IIa datasets. The error represents the full statistical and systematic uncertainty with which we know the data efficiency and fake rate for the samples on a per jet basis. [18]

In a similar way, the TRFc is calculated using a scale factor of c-tagging efficiency over b-tagging efficiency, as

$$
T R F c=T R F b \times \frac{\epsilon_{c}^{M C}}{\epsilon_{b}^{M C}}
$$

The TRFs are parameterized as functions of $p_{T}$ and $\eta$ for each operation point, as shown in Fig. 4.19 and Fig. 4.20.

A jet identified as good in the analysis is taggable only if it is matched to a track jet within $\Delta R=0.5$, where the track jet is reconstructed from a seed track with $p_{T}>1 \mathrm{GeV}$ and must contain at least 2 tracks, each with $p_{T}>0.5 \mathrm{GeV}$ consisting of at least 2 SMT hits and satisfying $\left|d c a_{x y}\right|<0.2 \mathrm{~cm}$ and $\left|d c a_{z}\right|<0.4 \mathrm{~cm}$. The taggability is defined as

$$
\text { Taggability }=\frac{\text { Taggable \&\& Good }}{\text { Good }} .
$$

Note that due to the higher luminosity in Run IIb data taking, more non-taggable jets from minimum bias events make the taggability more dependent on the instantaneous 

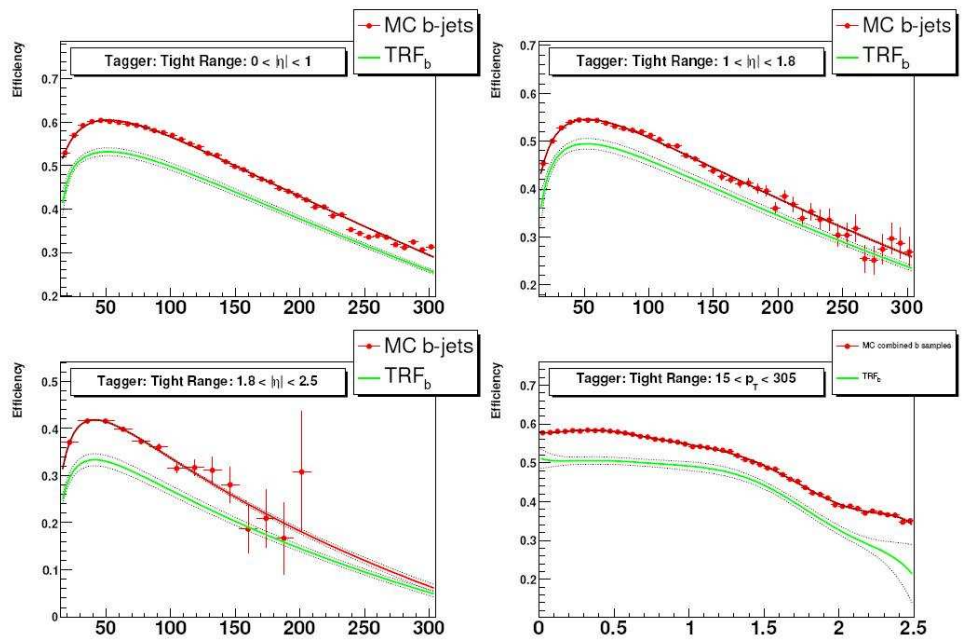

Figure 4.19: b-tagging efficiency as a function of $p_{T}$ or $\eta$ for the Tight operation point of the NN bjet tagger. It is determined on Run IIb datasets (red for MC and green for data) in the $\mathrm{CC}$, ICR and EC region respectively. The TRFb on data is obtained by multiplying the MC b-jet tagging efficiency by the scale factor. The dotted black lines are the fit errors dominated by the scale factor fit errors. [18]

luminosity. A new definition is thus introduced for Run IIb analysis, as

$$
\text { Taggability }=\frac{\text { Taggable \&\& Good \&\& } N_{t r k} \geq 2}{\text { Good \&\& } N_{t r k} \geq 2}
$$

where $N_{t r k}$ is number of tracks on the jet with $p_{T}>0.5 \mathrm{GeV},\left|d c a_{x y}\right|<0.5 \mathrm{~cm}$, $d c a_{z}<1.0 \mathrm{~cm}$ and $|\Delta z|<2.0 \mathrm{~cm}$.

To take into account of the difference between data and simulated events, the taggability scale factor (TSF) [77] is derived and applied to simulated events. In this analysis, TSF is parameterized as functions of $p_{T}$ and $\eta$, and determined separately in regions: $\mathrm{z}$ position of the primary vertex in $(-60 \mathrm{~cm}, 30 \mathrm{~cm}),(-30 \mathrm{~cm}, 0),(0,30 \mathrm{~cm})$ and $(30 \mathrm{~cm}, 60 \mathrm{~cm})$. A closure test is performed by plotting the ratio of data taggability to the corrected simulation taggability, as shown in Fig. 4.21. The fitted curves are flat at value of 1 , indicating that TSF are properly derived and applied on the simulated events.

In this analysis, the $\mathrm{NN}$ b-jet tagger is applied directly to the jets of simulated events. Therefore, the TSF and b-tagging efficiency scale factors are applied on 


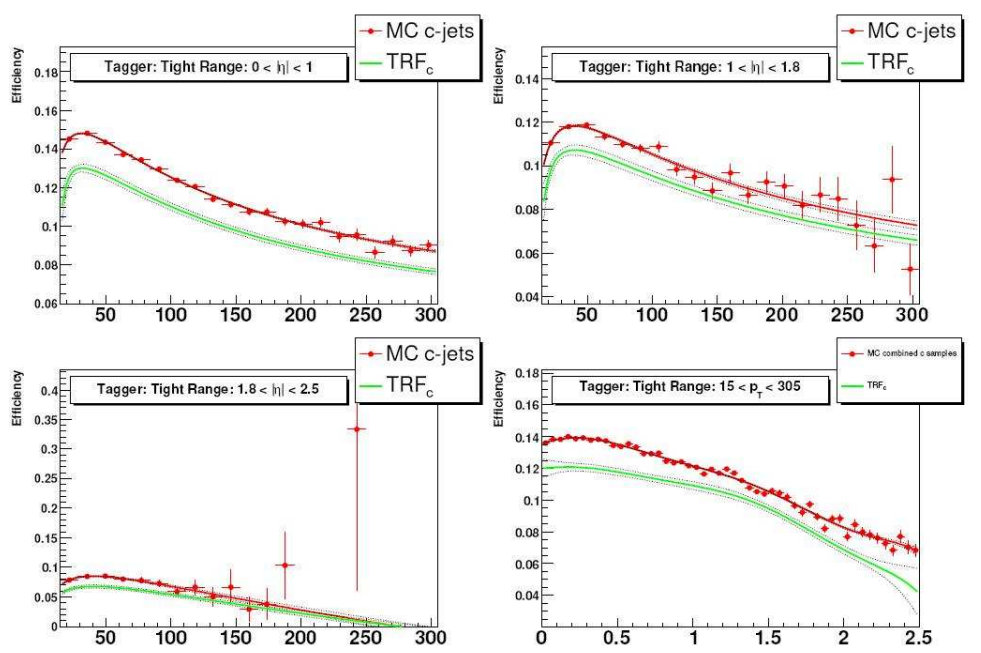

Figure 4.20: c jet tagging efficiency as a function of $p_{T}$ or $\eta$ for the Tight operation point of the NN tagger. It is determined on Run IIb datasets (red for MC and green for data) in the $\mathrm{CC}$, ICR and EC region respectively. The TRFc on data is obtained by multiplying the b-jet tagging efficiency by the $\mathrm{c} / \mathrm{b}$ scale factor. The dotted black lines are the fit errors dominated by the scale factor fit errors. [18]

simulated events to correct the difference. Note that the scale factor of tagging efficiency is only available for b and c jets. When it is applied to the mis-tagged light jets in event samples like $W j j$, overestimation of b jets could happen. This effect is estimated using $W j j$ event samples by comparing the result to the one predicted using only TRFs. The rescaling factors are determined to be 1.46 and 1.56 \pm 0.10 (stat. only) for Tight and oldLoose operating points. 

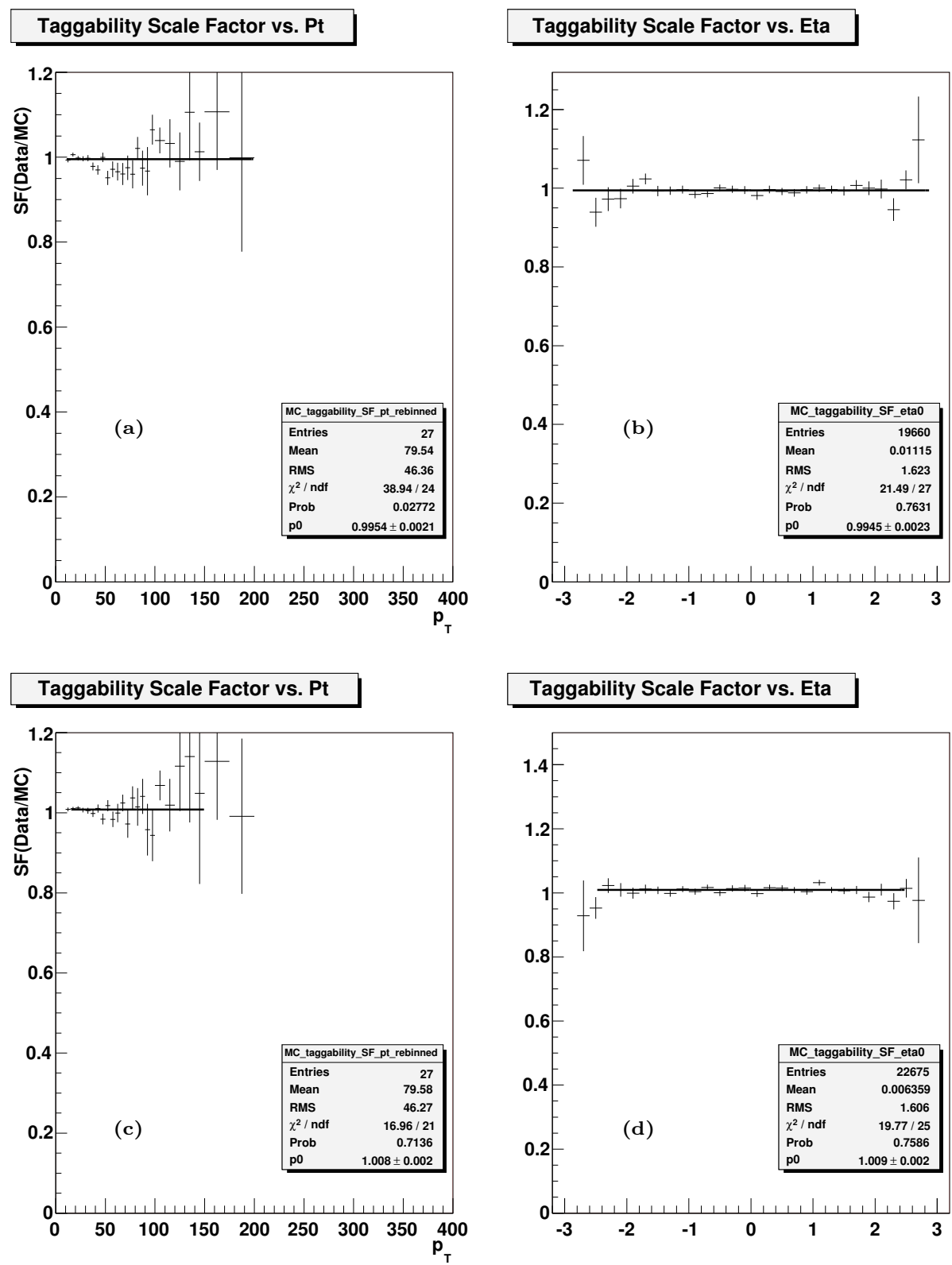

Taggability Scale Factor vs. Eta

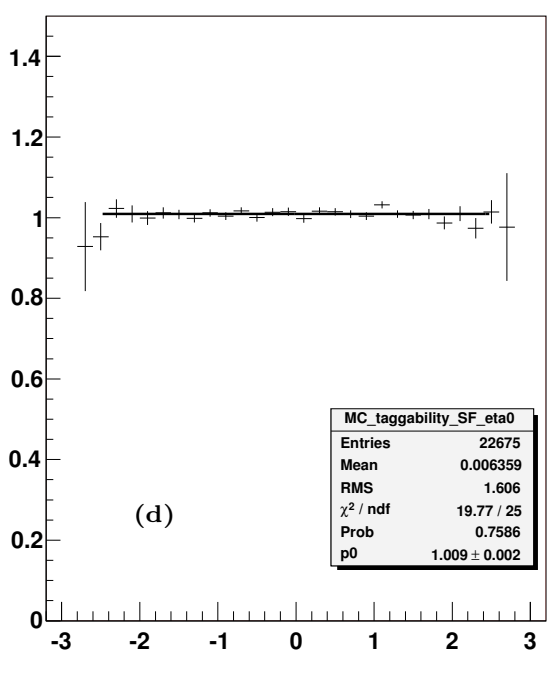

Figure 4.21: Closure test for p20 Jet taggability scale factors for electron and muon channel (a) Electron $p_{T}$ dependence, (b) Electron $\eta$ dependence, (c) Muon $p_{T}$ dependence and (d) Muon $\eta$ dependence. 


\section{CHAPTER V}

\section{The Datasets and Event Selection}

The analysis presented in this thesis is based on data collected at the upgraded DØ detector. In Section 5.1 the data samples with the corresponding triggers and luminosities are briefly described. The Monte Carlo simulated event samples, which correspond to the physics processes relevant to this analysis, are summarized as well. In order to optimize the Higgs boson signal significance, a variety of requirements are applied to the reconstructed events. The criteria and corrections are described in Section 5.2. The $\mathrm{D} \varnothing$ b-jet tagging algorithm is used to identify the b quarks from Higgs boson decay. The results are summarized in Section 5.3.

\subsection{Data Sample and Simulated Events}

\subsubsection{Data Sample, Luminosities and Triggers}

The data sample used in this analysis is collected at the D $\varnothing$ detector between April 2002 to April 2008 and corresponds to an integrated luminosity of $2.7 \mathrm{fb}^{-1}$ - It is divided into two subsets, called Run IIa and Run IIb, which are analyzed separately. The reason is that the detector responses are significantly different due to the upgrade of the $\mathrm{D} \varnothing$ detector in 2006. An additional inner layer silicon detector is added to the SMT to deliver better tracking, while the L1 calorimeter and silicon track triggers were upgraded in order to improve detector's performance with higher 


\begin{tabular}{|l|l|l|}
\hline & EMinclusive & MUinclusive \\
\hline \multirow{3}{*}{ Run IIa } & CSG_CAF_EMinclusive_PASS3_p17.09.03 & CSG_CAF_MUinclusive_PASS3_p17.09.03 \\
& CSG_CAF_EMinclusive_PASS3_p17.09.06 & CSG_CAF_MUinclusive_PASS3_p17.09.06 \\
& CSG_CAF_EMinclusive_PASS3_p17.09.06b & CSG_CAF_MUinclusive_PASS3_p17.09.06b \\
\hline \multirow{3}{*}{ Run IIb } & CSG_CAF_EMinclusive_PASS2_p21.05.00_all_fixed2007 & CSG_CAF_MUinclusive_PASS2_p21.05.00_allfixed2007 \\
& CSG_CAF_EMinclusive_PASS4_p21.08.00 & CSG_CAF_MUinclusive_PASS4_p21.10.00_p20.12.00 \\
& & CSG_CAF_MUinclusive_PASS4_p21.10.00_p20.12.01 \\
& & CSG_CAF_MUinclusive_PASS4_p21.10.00_p20.12.02_summer2008 \\
\hline
\end{tabular}

Table 5.1: Run IIa and Run IIb data samples used in the analysis.

luminosities.

The Run IIa dataset, collected between April 2002 and February 2006, corresponds to an integrated luminosity of $\sim 1.1 \mathrm{fb}^{-1}$. The events in this dataset are reconstructed using the $\mathrm{D} \varnothing$ offline reconstruction software $\mathrm{D} \varnothing$ reco [78] of versions $p 17 . x x . x x$, which take into account of differences caused by hadronic calibrations and cable swapping [79]. The Run IIb dataset corresponds to an integrated luminosity of $\sim 1.6 \mathrm{fb}^{-1}$. It was collected during the periods of June 2006 - August 2007 and October 2007 - April 2008, where the 3-month gap in 2007 is due to a shutdown of the Tevatron. The Run IIb datasets are reconstructed using DØreco of versions p20.xx.xx, with different versions developed to handle warm calorimeter cell and electron track match issues [80] [81].

Because the initial reconstructed data sample is too large to efficiently analyze, it is skimmed into smaller individual samples with different selection criteria corresponding to specific physics purposes [82]. In this analysis the EMinclusive and MUinclusive skims are used. The EMinclusive skim is required to contain at least one EM object with transverse momentum $p_{T}>8 \mathrm{GeV}$ per event, while the MUinclusive skim selects those events with at least one loose quality muon with transverse momentum $p_{T}>8 \mathrm{GeV}$ determined by the central tracking system. All the skimmed datasets in this analysis are summarized in Tab. 5.1.

The integrated luminosities corresponding to these data samples are calculated using the $l m \_a c c e s s$ package [83] created by the DØ Luminosity ID group [84]. The 


\begin{tabular}{|l|l|r|}
\hline List & Trigger & Luminosity $\mathrm{pb}^{-1}$ \\
\hline v8 & EM15_2JT15 & 23.35 \\
v9 & EM15_2JT15 & 24.73 \\
v10 & EM15_2JT15 & 9.81 \\
v11 & EM15_JT15 & 63.40 \\
v12 & E1_SHT15_2J20 & 227.35 \\
v13a & E1_SHT15_2J_J25 & 55.22 \\
v13b & E1_SHT15_2J_J30 & 298.21 \\
v14 & E1_SHT15_2J_J25 & 333.57 \\
\hline Run IIa & & Sub Total 1035.64 \\
\hline v15.0-V15.5 & JT_125_L3J125 & 534.5 \\
v15.5-V15.9 & JT_125_L3J125 & 693.8 \\
v15.9-V16.0 & JT_125_L3J125 & 392.3 \\
v16.0- & JT_125_L3J125 & 11.85 \\
\hline Run IIb & & Sub Total 1608.8 \\
\hline \hline & & Total 2644.4 \\
\hline
\end{tabular}

Table 5.2: Recorded integrated luminosities for the datasets in EM channel. The datasets correspond to different trigger list versions, which are used in different periods of $\mathrm{D} \varnothing$ data taking. The triggers in the table are unprescaled triggers.

measurement of luminosity is in the unit of luminosity block with a unique luminosity block number (LBN) [85]. Each luminosity block is a time span of 60 seconds, during which the instantaneous luminosity is considered to be effectively constant. Because L1 triggers have different responses to different non-uniform bunch-to-bunch luminosity profiles, several triggers with common dead time are grouped together to determine the luminosity [86]. The trigger terms used in this analysis for luminosity calculation are summarized in Tab. 5.2 and Tab. 5.3. The datasets are further divided into subsets according to their trigger versions, in which triggers are configured differently in $\mathrm{D} \varnothing$ data taking. The triggers used in the calculation are unprescaled, which means all the triggered events are recorded. After removing the datasets with bad data quality and LBNs due to hardware or operation failure, the total integrated luminosities are $2.64 \mathrm{fb}^{-1}$ and $2.78 \mathrm{fb}^{-1}$ in the EM and MU skims respectively. In this analysis these numbers are further reduced by $3 \%$ due to an inefficient calorimeter noise rejection.

The triggers for this analysis are chosen to optimize the acceptance of the Higgs 


\begin{tabular}{|l|l|r|}
\hline List & Trigger & Luminosity $\mathrm{pb}^{-1}$ \\
\hline v8-v10.3 & JT_125TT & 31.77 \\
v10.3-v12 & JT_125TT & 74.75 \\
v12-v13 & JT_125TT & 231.21 \\
v13-v13.2 & JT_125TT & 38.05 \\
v13.2-v14 & JT_125TT & 337.49 \\
v14-v14.6 & JT_125TT & 142.23 \\
v14.6-v15 & JT_125TT & 190.77 \\
\hline Run IIa & & Sub Total 1046.3 \\
\hline v15.0 - v15.22 & JT125_L3J125 & 227.21 \\
v15.22 -v15.5 & JT125_L3J125 & 307.29 \\
v15.5 - v15.6 & JT125_L3J125 & 232.48 \\
v15.6 - v15.9 & JT125_L3J125 & 461.32 \\
v15.9 - v16.0 & JT125_L3J125 & 392.35 \\
v16.0 - & JT125_L3J125 & 109.94 \\
\hline Run IIb & & Sub Total 1730.6 \\
\hline \hline & & Total 2776.9 \\
\hline
\end{tabular}

Table 5.3: Recorded integrated luminosities for the datasets in MU channel. The datasets correspond to different trigger list versions, which are used in different periods of $D \varnothing$ data taking. The triggers in the table are unprescaled triggers.

\begin{tabular}{l|l} 
period & Trigger term \\
\hline v8 - v14 & EM15_2JT15 E1_SHT15_2J20 E1_SHT15_2J_J25 E1_SHT15_2J_J30
\end{tabular}

Table 5.4: EM+JET triggers through trigger versions v8 - v14 in the Run IIa datasets.

boson events. A series of different triggers are selected for the different datasets. For the EM skims, the selected triggers generally require each event to have a good EM object. For Run IIa datasets, a combination of triggers called EM+JET and Single EM triggers are used. The EM+JET triggers are shown in Tab. 5.4, while the Single EM triggers are shown in Tab. 5.5. For Run IIb datasets, Single EM triggers are used, as listed in Tab. 5.6. The triggers in the tables are combined into logical OR, which means an event will pass the entire trigger selection if it satisfies any of the triggers. The efficiency of events passing these trigger requirements are approximately $\sim 95 \%(\sim 90 \%)$ in Run IIa (IIb) datasets.

In the analysis with the MU skims, a combination of all the $\mathrm{D} \emptyset$ triggers is explored to maximize the signal acceptance. These triggers includes MU+JETS, Single MU and multi-jet triggers and in combination have an efficiency close to $100 \%$. To 


\begin{tabular}{l|l} 
period & Trigger term \\
\hline v8-v11 & EM_MX EM_MX_SH EM_MX_EMFR8 EM_HI EM_HI_SH \\
& EM_HI_EMFR8 EM_HI_2EM5_SH EM_HI_2EM5 EM_HI_2EM5_EMFR8 \\
& EM_HI_F0 EM_HI_2EM5_F0 EM_MX_F0 EM_HI_SH_TR \\
& EM_HI_2EM5_SH_TR EM_MX_SH_TR \\
\hline v12 & E1_SHT20 E2_SHT20 E3_SHT20 E4_SHT20 E1_L50 E1_VL70 E1_L70 \\
& E1_SH30 E2_SH30 E3_SH30 E4_SH30 E1_T13L15 E1_T25VL30 \\
& E1_SHT15_TK13 E2_T13L15 E2_T25VL30 E2_SHT15_TK13 E3_T13L15 \\
& E3_T25VL30 E3_SHT15_TK13 E4_T13L15 E4_T25VL30 E4_SHT15_TK13 \\
& E6_T13L15 E5_SHT15_TK13 E6_SHT15_TK13 \\
\hline v13.0-v13.1 & E1_NC90 E5_SHT20 E6_SHT20 E7_SHT20 E8_SHT20 E9_SHT20 E5_SH30 \\
& E6_SH30 E7_SH30 E8_SH30 E9_SH30 E7_SHT15_TK13 E8_SHT15_TK13 \\
& E9_SHT15_TK13 E9_SHT8_ITK10 E5_T13L15 E7_T13L15 E8_T13L15 \\
& E9_T13L15 E8_IT7SHT8 E9_IT7SHT8 E8_SHT8_ITK10 \\
\hline v13.2-13.9 & E1_SHT22 E2_SHT22 E3_SHT22 E4_SHT22 E1_T13SH15 E2_T13SH15 \\
& E3_T13SH15 E4_T13SH15 E5_T13SH15 E6_T13SH15 E7_T13SH15 \\
& E8_T13SH15 E9_T13SH15 E1_T15L20 E2_T15L20 E3_T15L20 E4_T15L20 \\
& E5_T15L20 E6_T15L20 E7_T15L20 E8_T15L20 E9_T15L20 E8_IT10SHT10 \\
& E9_IT10SHT10 E8_SHT10_ITK10 E9_SHT10_ITK10 \\
\hline \multirow{2}{*}{ v14 } & E1_SHT25 E3_SHT25 E4_SHT25 E1_SH35 E3_SH35 E4_SH35 \\
& E1_ISHT22 E3_ISHT22 E4_ISHT22 E1_ISH30 E3_ISH30 E4_ISH30 \\
& 2CEM12_E15_SHT22 2CEM12_E15_SH30 2CEM6_E15_SHT22 2CEM6_E15_SH30 \\
& E13_SHT25 E17_SHT25 E18_SHT25 E19_SHT25 E20_SHT25 E21_SHT25 \\
& E13_SH30 E17_SH30 E18_SH30 E19_SH30 E20_SH30 E21_SH30 E13_ISHT22 \\
& E17_ISHT22 E18_ISHT22 E19_ISHT22 E20_ISHT22 E21_ISHT22 E1_ISHT22 \\
& E13_ISH30 E17_ISH30 E18_ISH30 E19_ISH30 E20_ISH30 E21_ISH30 \\
& E1_ISHT15_TK13 E3_ISHT15_TK13 E4_ISHT15_TK13 E13_ISHT15_TK13 \\
& E17_ISHT15_TK13 E18_ISHT15_TK13 E19_ISHT15_TK13 E20_ISHT15_TK13 \\
& E21_ISHT15_TK13 E1_T13SHT15 E3_T13SHT15 E4_T13SHT15 E13_T13SHT15 \\
& E17_T13SHT15 E18_T13SHT15 E19_T13SHT15 E20_T13SHT15 E21_T13SHT15 \\
& E1_T15SH20 E3_T15SH20 E4_T15SH20 E13_T15SH20 E17_T15SH20 \\
& E17_SHT12_ITK10 E21_SHT12_ITK10
\end{tabular}

Table 5.5: Single EM triggers through trigger versions v8 - v14 in the Run IIa datasets. 
\begin{tabular}{l|l} 
period & Trigger term \\
\hline v15 & E1 ThSHT
\end{tabular}

\begin{tabular}{|c|c|}
\hline v15 & $\begin{array}{l}\text { E1_T13SHT15, E1_T15SH20, E1_ISHT15_TK13 } \\
\text { E2_T13SHT15, E2_T15SH20, E2_ISHT15_TK13 } \\
\text { TE1_T13SHT15, TE1_T15SH20, TE1_ISHT15_TK13, TE1_ISHT22, } \\
\text { TE1_SHT25, TE1_ISH30, TE1_SH35, TE1_L70 } \\
\text { TE2_T13SHT15, TE2_T15SH20, TE2_ISHT15_TK13, TE2_ISHT22, } \\
\text { TE2_SHT25, TE2_ISH30, TE2_SH35, TE2_L70 } \\
\text { TE3_T13SHT15, TE3_T15SH20, TE3_ISHT15_TK13, TE3_ISHT22, } \\
\text { TE3_SHT25, TE3_ISH30, TE3_SH35, TE3_L70 } \\
\text { TE4_T13SHT15, TE4_T15SH20, TE4_ISHT15_TK13, TE4_ISHT22, } \\
\text { TE4_SHT25, TE4_ISH30, TE4_SH35, TE4_L70 } \\
\text { TE5_T13SHT15, TE5_T15SH20, TE5_ISHT15_TK13, TE5_ISHT22, } \\
\text { TE5_SHT25, TE5_ISH30, TE5_SH35, TE5_L70 }\end{array}$ \\
\hline v15.50 & $\begin{array}{l}\text { E1_LH2ISHT17T14, E1_T14LH2SH17 } \\
\text { E2_LH2ISHT17T14, E2_T14LH2SH17 } \\
\text { TE1_LH2SH27, TE1_LH2L70, TE1_SHT50, TE1_SH60, TE1_LH2L70, TE1_L80 } \\
\text { TE1_LH2ISH24, TE1_LH2ISHT17T14, TE1_T14LH2SH17 } \\
\text { TE2_LH2SH27, TE2_LH2L70, TE2_SHT50, TE1_SH60, TE2_LH2L70, TE2_L80 } \\
\text { TE2_LH2ISH24, TE2_LH2ISHT17T14, TE2_T14LH2SH17 } \\
\text { TE3_LH2SH27, TE3_LH2L70, TE3_SHT50, TE1_SH60, TE3_LH2L70, TE3_L80 } \\
\text { TE3_LH2ISH24, TE3_LH2ISHT17T14, TE3_T14LH2SH17 } \\
\text { TE4_LH2SH27, TE4_LH2L70, TE4_SHT50, TE1_SH60, TE4_LH2L70, TE4_L80 } \\
\text { TE4_LH2ISH24, TE4_LH2ISHT17T14, TE4_T14LH2SH17 } \\
\text { TE5_LH2SH27, TE5_LH2L70, TE5_SHT50, TE1_SH60, TE5_LH2L70, TE5_L80 } \\
\text { TE5_LH2ISH24, TE5_LH2ISHT17T14, TE5_T14LH2SH17 }\end{array}$ \\
\hline v16 & $\begin{array}{l}\text { E1_LH2ISHT17T14, E1_T14LH2SH17 } \\
\text { E2_LH2ISHT17T14, E2_T14LH2SH17 } \\
\text { TE1_LH2SH27_NOLUM, TE1_LH2L70_NOLUM, TE1_SHT50_NOLUM, } \\
\text { TE1_SH60_NOLUM, TE1_LH2L70_NOLUM, TE1_L80_NOLUM } \\
\text { TE1_LH2ISH24_NOLUM, TE1_LH2ISHT17T14_NOLUM, TE1_T14LH2SH17_NOLUM } \\
\text { TE2_LH2SH27, TE2_LH2L70, TE2_SHT50, TE1_SH60, TE2_LH2L70, TE2_L80 } \\
\text { TE2_LH2ISH24, TE2_LH2ISHT17T14, TE2_T14LH2SH17 } \\
\text { TE3_LH2SH27_NOLUM, TE3_LH2L70_NOLUM, TE3_SHT50_NOLUM, } \\
\text { TE1_SH60_NOLUM, TE3_LH2L70_NOLUM, TE3_L80_NOLUM } \\
\text { TE3_LH2ISH24_NOLUM, TE3_LH2ISHT17T14_NOLUM, TE3_T14LH2SH17_NOLUM } \\
\text { TE4_LH2SH27_NOLUM, TE4_LH2L70_NOLUM, TE4_SHT50_NOLUM, } \\
\text { TE1_SH60_NOLUM, TE4_LH2L70_NOLUM, TE4_L80_NOLUM } \\
\text { TE4_LH2ISH24,_NOLUM TE4_LH2ISHT17T14_NOLUM, TE4_T14LH2SH17_NOLUM } \\
\text { TE5_LH2SH27, TE5_LH2L70, TE5_SHT50, TE1_SH60, TE5_LH2L70, TE5_L80 } \\
\text { TE5_LH2ISH24, TE5_LH2ISHT17T14, TE5_T14LH2SH17 }\end{array}$ \\
\hline
\end{tabular}

Table 5.6: Single EM triggers through trigger versions v15-v16 in the Run IIb datasets. 
ensure there is no bias caused by combining all these triggers, a study with Single MU triggers is performed on both Run IIa and Run IIb datasets. For Run IIa datasets, the event triggering ratio of Single MU triggers, shown in Tab. 5.7, to all the triggers is $\sim 70 \%$, consistent with efficiency determined by the tag-and-probe method on di-muon events [87]. The uncertainty of event statistics due to this is $\sim 3 \%$. The shapes of the relevant kinematic distributions remain unchanged when using either Single MU triggers or all the D $\varnothing$ triggers. A similar study is made on the Run IIb datasets, and finally a conservative $4 \%$ error is added to the systematic uncertainty in this analysis.

\begin{tabular}{|c|c|c|c|}
\hline v8-11/v12 & v13 & v14 & v15 \\
\hline MUW_A_L2M3_TRK10 & MUH1_TK12_TLM12 & MUH1_TK12_TLM12 & MUHI1_ITLM10 \\
\hline MU_W_L2M5_TRK10 & MUH1_TK12 & MUH8_TK12_TLM12 & MUHI1_TK12_TLM12 \\
\hline MUW_W_L2M3_TRK10 & MUH1_LM15 & MUH1_ILM15 & MUHI1_ILM15 \\
\hline MU_W_L2M0_TRK3 & MUH1_TK10 & MUH1_ITLM10 & MUHI2_ITLM10 \\
\hline MU_W_L2M3_TRK10 & MUH2_LM3_TK12 & MUH8_ILM15 & MUHI2_TK12_TLM12 \\
\hline MUW_W_L2M5_TRK10 & MUH2_LM6_TK12 & MUH8_ITLM10 & MUHI2_ILM15 \\
\hline MU_W_L2M0_TRK10 & MUH2_LM10_TK12 & MUH5_LM15 & MUHI3_ITLM10 \\
\hline & MUH2_LM15 & MUH6_TK12_TLM12 & MUHI3_TK12_TLM12 \\
\hline & MUH3_LM3_TK10 & MUH6_LM15 & MUHI3_ILM15 \\
\hline & MUH3_LM6_TK12 & MUH7_TK12 & \\
\hline & MUH3_LM10_TK12 & MUH7_LM15 & \\
\hline & MUH3_LM15 & & \\
\hline & MUH4_LM15 & & \\
\hline & MUH4_TK10 & & \\
\hline & MUH5_LM15 & & \\
\hline & MUH6_TK12_TLM12 & & \\
\hline & MUH6_LM15 & & \\
\hline & MUH6_TK10 & & \\
\hline & MUH7_TK12 & & \\
\hline & MUH7_LM15 & & \\
\hline & MUH7_TK10 & & \\
\hline
\end{tabular}

Table 5.7: List of Single MU triggers used in the trigger study on Run IIa datasets.

\subsubsection{Simulated Event Samples}

\section{Monte Carlo Event Generators}

A good understanding of the relevant physical processes and detector responses heavily relies on the simulation of these events. In high energy hadron collisions, the 
physical processes are well described by the parton model. Hadrons are considered to be composed of seas of point-like partons, referred as quarks and leptons, which carry different fractions of incoming longitudinal momenta. The parton distribution function, which is a probability density for a parton to carry a momentum fraction $x$ at momentum transfer scale $Q^{2}$, is empirically determined and parameterized in software packages such as CTEQ [88]. In the case of proton-antiproton collisions, the physical processes are simulated in the stages of initial state radiation, parton hard scattering, final state radiation and parton hadronization. The initial and final state radiations often refers to parton shower in which high momentum partons evolves to a cascade of partons with momentum decreasing to the cutoff value. The parton scattering is the hard interactions of the high momentum partons. The resulting partons finally undergo fragmentation process into hadrons.

In this analysis several popular Monte Carlo (MC) event generators such as PYTHIA [89], ALPGEN [90] and CompHEP [91] are jointly used to simulate the related physical processes. The PYTHIA program is a general purpose event generator for $p p, p \bar{p}, e^{+} e^{-}$and $\mu^{+} \mu^{-}$collisions. Its initial and final state parton showering algorithms are based on the $p_{T}$ ordered shower evolution, where parton shower is described by the DGLAP equations [92] [93] [94]. The hadronization process is exclusively based on the Lund string fragmentation framework [95], where quark and antiquark are paired with a string. It can simulate numerous hard processes such as vector boson production and the Higgs production, as well as those soft processes like diffractive and elastic scattering. However, PYTHIA is optimized for $2 \rightarrow 1$ and $2 \rightarrow 2$ processes and not suitable to handle those processes with at least three particles in the final state.

$A L P G E N$ is an event generator dedicated for hard multi-parton processes in 
hadron collisions. It calculates the exact matrix elements for selected parton-level processes at leading order QCD and Electroweak interactions. It provides a good description of the hard interactions with multiple final state partons. $A L P G E N$ is not available to carry out parton shower and hadronization but includes an interface to PYTHIA. Thus, ALPGEN and PYTHIA are combined to generate the desired events through a so-called MLM matching scheme [96]. The events are generated using $A L P G E N$ with exclusive parton multiplicity such $W+3$ light partons, which in turn are input to PYTHIA for showering and hadronizing into jets. The partons and jets are matched one to one, and all the events from each light parton multiplicity processes are summed up according to their cross sections. The SingleTop generator [97] based on the CompHEP package is specialized to simulate the electroweak top quark production at hadron colliders in the NLO approximation. It is also interfaced to PYTHIA for parton showering.

\section{Simulated Event Samples}

The events of Run IIa and Run IIb are separately simulated due to different luminosities and detector responses. The simulated event samples along with the corresponding $\mathrm{MC}$ event generators, number of generated events and production cross sections are summarized in Tab. 5.8 - 5.12. As shown in Tab. 5.8, the Higgs boson events are generated solely with PYTHIA using PDFs from CTEQ6L. The $Z H$ events are also included in the signal taking into account their $\sim 5 \%$ contribution to the total number of $W H$ events passing the events selection. Diboson events, shown in Tab. 5.9, are produced exclusively with PYTHIA as well. The top quark productions shown in Tab. 5.10 and $W+$ jets events shown Tab. 5.115 .12 are produced with CompHEP or ALPGEN using PYTHIA for parton shower and hadronization. 


\begin{tabular}{|c|c|c|c|c|c|}
\hline data & Process & & Generator & \# events & $\sigma(\times \mathrm{BR})[\mathrm{pb}]$ \\
\hline \multirow{11}{*}{ Run IIa } & \multirow{11}{*}{$H W \rightarrow b \bar{b}+\ell \nu$} & \multirow{11}{*}{$\begin{array}{l}m_{H}=100 \mathrm{GeV} \\
m_{H}=105 \mathrm{GeV} \\
m_{H}=110 \mathrm{GeV} \\
m_{H}=115 \mathrm{GeV} \\
m_{H}=120 \mathrm{GeV} \\
m_{H}=125 \mathrm{GeV} \\
m_{H}=130 \mathrm{GeV} \\
m_{H}=135 \mathrm{GeV} \\
m_{H}=140 \mathrm{GeV} \\
m_{H}=145 \mathrm{GeV} \\
m_{H}=150 \mathrm{GeV}\end{array}$} & & $336.7 \mathrm{k}$ & \multirow{11}{*}{$\begin{array}{l}0.0266 \\
0.0222 \\
0.0183 \\
0.0150 \\
0.0119 \\
0.0093 \\
0.0069 \\
0.0050 \\
0.0034 \\
0.0022 \\
0.0013\end{array}$} \\
\hline & & & & $188.1 \mathrm{k}$ & \\
\hline & & & & $333.8 \mathrm{k}$ & \\
\hline & & & & $187.8 \mathrm{k}$ & \\
\hline & & & & $329.3 \mathrm{k}$ & \\
\hline & & & PYTHIA & $187.8 \mathrm{k}$ & \\
\hline & & & & $334.2 \mathrm{k}$ & \\
\hline & & & & $186.2 \mathrm{k}$ & \\
\hline & & & & $328.1 \mathrm{k}$ & \\
\hline & & & & $175.0 \mathrm{k}$ & \\
\hline & & & & $329.6 \mathrm{k}$ & \\
\hline \multirow{11}{*}{ Run IIa } & \multirow{11}{*}{$H Z \rightarrow b \bar{b}+\ell \ell$} & \multirow{11}{*}{$\begin{array}{l}m_{H}=100 \mathrm{GeV} \\
m_{H}=105 \mathrm{GeV} \\
m_{H}=110 \mathrm{GeV} \\
m_{H}=115 \mathrm{GeV} \\
m_{H}=120 \mathrm{GeV} \\
m_{H}=125 \mathrm{GeV} \\
m_{H}=130 \mathrm{GeV} \\
m_{H}=135 \mathrm{GeV} \\
m_{H}=140 \mathrm{GeV} \\
m_{H}=145 \mathrm{GeV} \\
m_{H}=150 \mathrm{GeV}\end{array}$} & & $330.7 \mathrm{k}$ & \multirow{11}{*}{$\begin{array}{r}0.0046 \\
0.00387 \\
0.00323 \\
0.00266 \\
0.00213 \\
0.00167 \\
0.00127 \\
0.00091 \\
0.000633 \\
0.00041 \\
0.000267 \\
\end{array}$} \\
\hline & & & & $187.3 \mathrm{k}$ & \\
\hline & & & & $333.2 \mathrm{k}$ & \\
\hline & & & & $187.6 \mathrm{k}$ & \\
\hline & & & & $328.4 \mathrm{k}$ & \\
\hline & & & PYTHIA & $175.9 \mathrm{k}$ & \\
\hline & & & & $327.4 \mathrm{k}$ & \\
\hline & & & & $187.3 \mathrm{k}$ & \\
\hline & & & & $317.9 \mathrm{k}$ & \\
\hline & & & & $187.5 \mathrm{k}$ & \\
\hline & & & & $333.7 \mathrm{k}$ & \\
\hline \multirow{11}{*}{ Run IIb } & \multirow{11}{*}{$H W \rightarrow b \bar{b}+\ell \nu$} & \multirow{11}{*}{$\begin{array}{l}m_{H}=100 \mathrm{GeV} \\
m_{H}=105 \mathrm{GeV} \\
m_{H}=110 \mathrm{GeV} \\
m_{H}=115 \mathrm{GeV} \\
m_{H}=120 \mathrm{GeV} \\
m_{H}=125 \mathrm{GeV} \\
m_{H}=130 \mathrm{GeV} \\
m_{H}=135 \mathrm{GeV} \\
m_{H}=140 \mathrm{GeV} \\
m_{H}=145 \mathrm{GeV} \\
m_{H}=150 \mathrm{GeV}\end{array}$} & & $317.1 \mathrm{k}$ & \multirow{11}{*}{$\begin{array}{l}0.0266 \\
0.0222 \\
0.0183 \\
0.0150 \\
0.0119 \\
0.0093 \\
0.0069 \\
0.0050 \\
0.0034 \\
0.0022 \\
0.0013\end{array}$} \\
\hline & & & & $291.8 \mathrm{k}$ & \\
\hline & & & & $317.4 \mathrm{k}$ & \\
\hline & & & & $278.6 \mathrm{k}$ & \\
\hline & & & & $314.6 \mathrm{k}$ & \\
\hline & & & PYTHIA & $277.3 \mathrm{k}$ & \\
\hline & & & & $324.1 \mathrm{k}$ & \\
\hline & & & & $278.1 \mathrm{k}$ & \\
\hline & & & & $323.9 \mathrm{k}$ & \\
\hline & & & & $289.2 \mathrm{k}$ & \\
\hline & & & & $320.4 \mathrm{k}$ & \\
\hline \multirow{11}{*}{ Run IIb } & \multirow{11}{*}{$H Z \rightarrow b \bar{b}+\ell \ell$} & \multirow{11}{*}{$\begin{aligned} m_{H} & =100 \mathrm{GeV} \\
m_{H} & =105 \mathrm{GeV} \\
m_{H} & =110 \mathrm{GeV} \\
m_{H} & =115 \mathrm{GeV} \\
m_{H} & =120 \mathrm{GeV} \\
m_{H} & =125 \mathrm{GeV} \\
m_{H} & =130 \mathrm{GeV} \\
m_{H} & =135 \mathrm{GeV} \\
m_{H} & =140 \mathrm{GeV} \\
m_{H} & =145 \mathrm{GeV} \\
m_{H} & =150 \mathrm{GeV}\end{aligned}$} & & $320.7 \mathrm{k}$ & \multirow{11}{*}{$\begin{array}{r}0.0046 \\
0.00387 \\
0.00323 \\
0.00266 \\
0.00213 \\
0.00167 \\
0.00127 \\
0.00091 \\
0.000633 \\
0.00041 \\
0.000267 \\
\end{array}$} \\
\hline & & & & $275.2 \mathrm{k}$ & \\
\hline & & & & $305.7 \mathrm{k}$ & \\
\hline & & & & $279.4 \mathrm{k}$ & \\
\hline & & & & $310.2 \mathrm{k}$ & \\
\hline & & & PYTHIA & $274.3 \mathrm{k}$ & \\
\hline & & & & $331.0 \mathrm{k}$ & \\
\hline & & & & $278.4 \mathrm{k}$ & \\
\hline & & & & $321.3 \mathrm{k}$ & \\
\hline & & & & $278.4 \mathrm{k}$ & \\
\hline & & & & $317.3 \mathrm{k}$ & \\
\hline
\end{tabular}

Table 5.8: The simulated samples of signal events $p \bar{p} \rightarrow W+H \rightarrow l \nu+b \bar{b}$ and $p \bar{p} \rightarrow Z+H \rightarrow l l+b \bar{b}$. The MC event generators, number of generated events and cross sections are shown in the table for Run IIa and Run IIb respectively. The branching ratios used in the calculation is for only one lepton flavor. 


\begin{tabular}{|c|l|l|r|r|}
\hline data & Process & Generator & \# events & $\sigma \times \mathrm{BR}[\mathrm{pb}]$ \\
\hline \hline \multirow{5}{*}{ Run IIa } & $W W \rightarrow e \nu j j$ & PYTHIA & $87 \mathrm{k}$ & $12.0 \times 0.146$ \\
& $W W \rightarrow \mu \nu j j$ & PYTHIA & $107 \mathrm{k}$ & $12.0 \times 0.146$ \\
& $W Z \rightarrow e \nu j j$ & PYTHIA & $96 \mathrm{k}$ & $3.68 \times 0.075$ \\
& $W Z \rightarrow \mu \nu j j$ & PYTHIA & $47 \mathrm{k}$ & $3.68 \times 0.075$ \\
& $W Z \rightarrow j j e e$ & PYTHIA & $92 \mathrm{k}$ & $3.68 \times 0.023$ \\
& $W Z \rightarrow j j \mu \mu$ & PYTHIA & $96 \mathrm{k}$ & $3.68 \times 0.023$ \\
& $Z Z$ inclusive & PYTHIA & $95 \mathrm{k}$ & 1.43 \\
\hline \multirow{5}{*}{ Run IIb } & $W W$ inclusive & PYTHIA & $703.3 \mathrm{k}$ & 12.0 \\
& $W Z$ inclusive & PYTHIA & $638.9 \mathrm{k}$ & 3.68 \\
& $Z Z$ inclusive & PYTHIA & $538.8 \mathrm{k}$ & 1.42 \\
\hline
\end{tabular}

Table 5.9: The simulated samples of the diboson processes $W W, W Z$ and $Z Z$. The MC event generators, number of generated events and cross sections are shown in the table for Run IIa and Run IIb respectively. A combination of several exclusive channels is used for Run IIa, while the inclusive samples are used for Run IIb.

\begin{tabular}{|c|c|c|c|c|}
\hline Run IIa MC & & Generator & \# events & $K \times(\sigma \times \mathrm{BR})[\mathrm{pb}]$ \\
\hline \multirow{3}{*}{$t \bar{t} \rightarrow b \bar{b}+\ell^{+} \nu \ell^{\prime-} \bar{\nu}_{\ell^{\prime}}$} & + + 0 light parton & \multirow{3}{*}{ ALPGEN + PYTHIA } & $224 \mathrm{k}$ & $1.39 \times 0.3241$ \\
\hline & +1 light parton & & $96 \mathrm{k}$ & $1.39 \times 0.1348$ \\
\hline & +2 light partons & & $50 \mathrm{k}$ & $1.39 \times 0.0664$ \\
\hline \multirow{3}{*}{$t \bar{t} \rightarrow b \bar{b}+2 j+\ell \nu$} & +0 light parton & \multirow{3}{*}{ ALPGEN + PYTHIA } & $283 \mathrm{k}$ & $1.39 \times 1.2988$ \\
\hline & +1 light parton & & $98 \mathrm{k}$ & $1.39 \times 0.5407$ \\
\hline & +2 light partons & & $93 \mathrm{k}$ & $1.39 \times 0.2659$ \\
\hline \multicolumn{2}{|c|}{ Single-top $s$-channel $(t b \rightarrow \ell \nu b b)$} & CompHEP + PYTHIA & $291 \mathrm{k}$ & 0.0978 \\
\hline \multicolumn{2}{|c|}{ Single-top $t$-channel $(t q b \rightarrow \ell \nu b q b)$} & CompHEP + PYTHIA & $385 \mathrm{k}$ & 0.22 \\
\hline Run IIb MC & & Generator & \# events & $\overline{\bar{K} \times(\sigma \times \mathrm{BR})[\mathrm{pb}]}$ \\
\hline \multirow{3}{*}{$t \bar{t} \rightarrow b \bar{b}+\ell^{+} \nu \ell^{\prime-} \bar{\nu}_{\ell^{\prime}}$} & +0 light parton & \multirow{3}{*}{ ALPGEN + PYTHIA } & 274275 & 1.39×0.3241 \\
\hline & +1 light parton & & 131978 & $1.39 \times 0.1348$ \\
\hline & +2 light partons & & 66315 & $1.39 \times 0.0664$ \\
\hline \multirow{3}{*}{$t \bar{t} \rightarrow b \bar{b}+2 j+\ell \nu$} & +0 light parton & \multirow{3}{*}{ ALPGEN + PYTHIA } & 743325 & $1.39 \times 1.2988$ \\
\hline & +1 light parton & & 415698 & $1.39 \times 0.5407$ \\
\hline & +2 light partons & & 162630 & $1.39 \times 0.2659$ \\
\hline \multicolumn{2}{|c|}{ Single-top $s$-channel $(t b \rightarrow e \nu b b)$} & CompHEP + PYTHIA & 247003 & 0.0978 \\
\hline \multirow{2}{*}{\multicolumn{2}{|c|}{$\begin{array}{l}\text { Single-top } s \text {-channel }(t b \rightarrow \mu \nu b \bar{b}) \\
\text { Single-top } s \text {-channel }(t b \rightarrow \tau \nu b \bar{b})\end{array}$}} & CompHEP + PYTHIA & 261833 & 0.0978 \\
\hline & & CompHEP + PYTHIA & 194653 & 0.0978 \\
\hline \multicolumn{2}{|c|}{ Single-top $t$-channel $(t q b \rightarrow e \nu b q b)$} & CompHEP + PYTHIA & 262608 & 0.22 \\
\hline \multicolumn{2}{|c|}{ Single-top $t$-channel $(t q b \rightarrow \mu \nu b q b)$} & CompHEP + PYTHIA & 244905 & 0.22 \\
\hline \multicolumn{2}{|c|}{ Single-top $t$-channel $(t q b \rightarrow \tau \nu b q b)$} & CompHEP + PYTHIA & 260574 & 0.22 \\
\hline
\end{tabular}

Table 5.10: The simulated samples of top pair and electroweak single top productions. The MC event generators, number of generated events and cross sections are shown in the table for Run IIa and Run IIb respectively. The $\mathrm{K}$ factor for $t \bar{t}$ scales the $t \bar{t}$ cross section to $6.8 \mathrm{pb}$. No $\mathrm{K}$ factor is given for single top since the cross section given is already at NLO. 


\begin{tabular}{|c|c|c|c|c|}
\hline Run IIa MC Proc & & Generator & \# events & $\sigma(\times \mathrm{BR})[\mathrm{pb}]$ \\
\hline \multirow{6}{*}{$W j j \rightarrow \ell \nu$} & \multirow{6}{*}{$\begin{array}{l}+0 \text { light parton } \\
+1 \text { light parton } \\
+2 \text { light partons } \\
+3 \text { light partons } \\
+4 \text { light partons } \\
+5 \text { light partons }\end{array}$} & \multirow{6}{*}{ ALPGEN + PYTHIA } & $2.3 \mathrm{M}$ & $4=4574.4$ \\
\hline & & & $2.8 \mathrm{M}$ & 1273.9 \\
\hline & & & $1.6 \mathrm{M}$ & 298.6 \\
\hline & & & $789 \mathrm{k}$ & 70.6 \\
\hline & & & $779 \mathrm{k}$ & 15.8 \\
\hline & & & $58 \mathrm{k}$ & 5.6 \\
\hline \multirow{4}{*}{$W b \bar{b} \rightarrow \ell \nu b \bar{b}$} & \multirow{4}{*}{$\begin{array}{l}+0 \text { light parton } \\
+1 \text { light parton } \\
+2 \text { light partons } \\
+3 \text { light partons }\end{array}$} & \multirow{4}{*}{ ALPGEN + PYTHIA } & $740 \mathrm{k}$ & 19.2 \\
\hline & & & $261 \mathrm{k}$ & 7.9 \\
\hline & & & $171 \mathrm{k}$ & 2.6 \\
\hline & & & $164 \mathrm{k}$ & 1.1 \\
\hline \multirow{3}{*}{$W c \bar{c} \rightarrow \ell \nu c \bar{c}$} & \multirow{3}{*}{$\begin{array}{l}+0 \text { light parton } \\
+1 \text { light parton } \\
+2 \text { light partons }\end{array}$} & \multirow{3}{*}{ ALPGEN + PYTHIA } & $482 \mathrm{k}$ & 71.1 \\
\hline & & & $336 \mathrm{k}$ & 29.9 \\
\hline & & & $372 \mathrm{k}$ & 14.1 \\
\hline \multirow{4}{*}{$Z j j \rightarrow e e$} & \multirow{4}{*}{$\begin{array}{l}+0 \text { light parton } \\
+1 \text { light parton } \\
+2 \text { light partons } \\
+3 \text { light partons }\end{array}$} & \multirow{4}{*}{ ALPGEN + PYTHIA } & $1.0 \mathrm{M}$ & 139.2 \\
\hline & & & $187 \mathrm{k}$ & 41.8 \\
\hline & & & $93 \mathrm{k}$ & 10.3 \\
\hline & & & $93 \mathrm{k}$ & 5.3 \\
\hline \multirow{4}{*}{$Z j j \rightarrow \mu \mu$} & \multirow{4}{*}{$\begin{array}{l}+0 \text { light parton } \\
+1 \text { light parton } \\
+2 \text { light partons } \\
+3 \text { light partons }\end{array}$} & \multirow{4}{*}{ ALPGEN + PYTHIA } & $839 \mathrm{k}$ & 139.5 \\
\hline & & & $209 \mathrm{k}$ & 41.6 \\
\hline & & & $104 \mathrm{k}$ & 10.3 \\
\hline & & & $104 \mathrm{k}$ & 5.3 \\
\hline \multirow{4}{*}{$Z j j \rightarrow \tau \tau$} & \multirow{4}{*}{$\begin{array}{l}+0 \text { light parton } \\
+1 \text { light parton } \\
+2 \text { light partons } \\
+3 \text { light partons }\end{array}$} & \multirow{4}{*}{ ALPGEN + PYTHIA } & $795 \mathrm{k}$ & 139.4 \\
\hline & & & $209 \mathrm{k}$ & 41.7 \\
\hline & & & $97 \mathrm{k}$ & 10.3 \\
\hline & & & $104 \mathrm{k}$ & 5.3 \\
\hline \multirow{3}{*}{$Z b \bar{b} \rightarrow \ell^{+} \ell^{-}+b \bar{b}$} & \multirow{3}{*}{$\begin{array}{l}+0 \text { light parton } \\
+1 \text { light parton } \\
+2 \text { light partons }\end{array}$} & \multirow{3}{*}{ ALPGEN + PYTHIA } & $604 \mathrm{k}$ & 0.97 \\
\hline & & & $271 \mathrm{k}$ & 0.36 \\
\hline & & & $144 \mathrm{k}$ & 0.21 \\
\hline \multirow{3}{*}{$Z c \bar{c} \rightarrow \ell^{+} \ell^{-}+c \bar{c}$} & \multirow{3}{*}{$\begin{array}{l}+0 \text { light parton } \\
+1 \text { light parton } \\
+2 \text { light partons }\end{array}$} & \multirow{3}{*}{ ALPGEN + PYTHIA } & $152 \mathrm{k}$ & 3.0 \\
\hline & & & $143 \mathrm{k}$ & 1.06 \\
\hline & & & $172 \mathrm{k}$ & 0.6 \\
\hline
\end{tabular}

Table 5.11: The simulated samples of $W+$ jets and $Z+j e t s$ for Run IIa. The MC event generators, number of generated events and cross sections are shown in the table. The samples are generated in bins of light parton multiplicity. Each bin is considered exclusive with a certain number of partons, except that the last bin is inclusive with more partons. The light partons are required to have $p_{T}>8 \mathrm{GeV}$ and $|\eta|<5$. The $l$ is inclusive to be $e$, $\mu$ and $\tau$. 


\begin{tabular}{|c|c|c|c|c|}
\hline Run IIb MC Proc & & Generator & \# events & $\sigma(\times \mathrm{BR})[\mathrm{pb}]$ \\
\hline \multirow{6}{*}{$W j j \rightarrow \ell \nu$} & \multirow{6}{*}{$\begin{array}{l}+0 \text { light parton } \\
+1 \text { light parton } \\
+2 \text { light partons } \\
+3 \text { light partons } \\
+4 \text { light partons } \\
+5 \text { light partons }\end{array}$} & \multirow{6}{*}{ ALPGEN + PYTHIA } & $4 \quad 4888.698$ & 40 \\
\hline & & & 3537.987 & 1279 \\
\hline & & & 2326.298 & 302 \\
\hline & & & 1091.511 & 71.7 \\
\hline & & & 1035.228 & 16.3 \\
\hline & & & 167.364 & 4.97 \\
\hline \multirow{4}{*}{$W b \bar{b} \rightarrow \ell \nu b \bar{b}$} & \multirow{4}{*}{$\begin{array}{l}+0 \text { light parton } \\
+1 \text { light parton } \\
+2 \text { light partons } \\
+3 \text { light partons }\end{array}$} & \multirow{4}{*}{ ALPGEN + PYTHIA } & $1368 \mathrm{k}$ & 9.36 \\
\hline & & & $1034 \mathrm{k}$ & 4.26 \\
\hline & & & $584.7 \mathrm{k}$ & 1.54 \\
\hline & & & $422.3 \mathrm{k}$ & 0.74 \\
\hline \multirow{3}{*}{$W c \bar{c} \rightarrow \ell \nu c \bar{c}$} & \multirow{3}{*}{$\begin{array}{l}+0 \text { light parton } \\
+1 \text { light parton } \\
+2 \text { light partons }\end{array}$} & \multirow{3}{*}{ ALPGEN + PYTHIA } & $1056 \mathrm{k}$ & 24.0 \\
\hline & & & $924.9 \mathrm{k}$ & 13.4 \\
\hline & & & $551.2 \mathrm{k}$ & 5.39 \\
\hline & +3 light partons & & $456.4 \mathrm{k}$ & 2.50 \\
\hline \multirow{4}{*}{$Z j j \rightarrow e e$} & \multirow{4}{*}{$\begin{array}{l}+0 \text { light parton } \\
+1 \text { light parton } \\
+2 \text { light partons } \\
+3 \text { light partons }\end{array}$} & \multirow{4}{*}{ ALPGEN + PYTHIA } & $1222 \mathrm{k}$ & 133.0 \\
\hline & & & $614.0 \mathrm{k}$ & 40.7 \\
\hline & & & 333.3 & 9.93 \\
\hline & & & $194.6 \mathrm{k}$ & 3.2 \\
\hline \multirow{4}{*}{$Z j j \rightarrow \mu \mu$} & \multirow{4}{*}{$\begin{array}{l}+0 \text { light parton } \\
+1 \text { light parton } \\
+2 \text { light partons } \\
+3 \text { light partons }\end{array}$} & \multirow{4}{*}{ ALPGEN + PYTHIA } & $1200 \mathrm{k}$ & 133.0 \\
\hline & & & $569.7 \mathrm{k}$ & 40.7 \\
\hline & & & 137630 & 9.93 \\
\hline & & & $380.5 \mathrm{k}$ & 3.2 \\
\hline \multirow{4}{*}{$Z j j \rightarrow \tau \tau$} & \multirow{4}{*}{$\begin{array}{l}+0 \text { light parton } \\
+1 \text { light parton } \\
+2 \text { light partons } \\
+3 \text { light partons }\end{array}$} & \multirow{4}{*}{ ALPGEN + PYTHIA } & $1088 \mathrm{k}$ & 133.0 \\
\hline & & & $693.8 \mathrm{k}$ & 40.7 \\
\hline & & & 342251 & 9.93 \\
\hline & & & $238.4 \mathrm{k}$ & 3.2 \\
\hline \multirow{3}{*}{$Z b \bar{b} \rightarrow \ell^{+} \ell^{-}+b \bar{b}$} & \multirow{3}{*}{$\begin{array}{l}+0 \text { light parton } \\
+1 \text { light parton } \\
+2 \text { light partons }\end{array}$} & \multirow{3}{*}{ ALPGEN + PYTHIA } & $185.3 \mathrm{k}$ & 0.418 \\
\hline & & & 87383 & 0.190 \\
\hline & & & $41.3 \mathrm{k}$ & 0.010 \\
\hline \multirow{3}{*}{$Z c \bar{c} \rightarrow \ell^{+} \ell^{-}+c \bar{c}$} & \multirow{3}{*}{$\begin{array}{l}+0 \text { light parton } \\
+1 \text { light parton } \\
+2 \text { light partons }\end{array}$} & \multirow{3}{*}{ ALPGEN + PYTHIA } & $182.4 \mathrm{k}$ & 0.930 \\
\hline & & & 84760 & 0.503 \\
\hline & & & 44.3 & 0.281 \\
\hline
\end{tabular}

Table 5.12: The simulated samples of $W+$ jets and $Z+j e t s$ for Run IIb. The MC event generators, number of generated events and cross sections are shown in the table. The samples are generated in bins of light parton multiplicity. Each bin is considered exclusive with a certain number of partons, except that the last bin is inclusive with more partons. The light partons are required to have $p_{T}>8 \mathrm{GeV}$ and $|\eta|<5$. The $l$ is inclusive to be $e$, $\mu$ and $\tau$. 
The cross sections used in the analysis are multiplied by the corresponding branching ratios and $K$ factors. The NNLO cross sections are used for the Higgs signal processes reducing the NLO cross sections by a factor of $\sim 0.96$. The cross sections of top pair production are scaled at NNLO to $\sim 6.8 \mathrm{pb}$ as well. The other samples are all scaled to the used NLO cross sections. Note that $W+$ jets and $Z+j$ ets are processed in the same way in this analysis. Both of these events undergo a special process called heavy flavor skimming, where events with additional heavy flavor partons generated in PYTHI A are removed to avoid double counting of these events.

\section{DØ Detector Simulation}

The generated events are passed through full simulation of the D $\varnothing$ detector using the software packages DØgstar [98] and DØSim [99]. DØgstar, namely DØ GEANT Simulation of the Total Apparatus Response, simulates the responses of the detector components to the generated events by depositing energies and leaving tracks. Based on the simulation toolkit GEANT [100] at CERN, the package is capable of describing the geometry and materials of the $\mathrm{D} \varnothing$ detector systems. It simulates the passage of particles interacting with tracking detectors, calorimeters and muon chambers as well as the solenoidal and toridal magnetic fields.

DØSim uses the output of DØgstar as input to generate the raw data. It simulates the digitization of electronics readout, detector noises and inefficiencies, as well as underlying events. In order to simulate the additional inelastic $p \bar{p}$ events, the generated events are overlaid with minimum bias events of real data triggered by the luminosity detector. The output of DØSim is in the form of raw data chunks which are later processed offline through $\mathrm{D} \varnothing$ reco just like real data to reconstruct the events. 


\subsection{The Event Selection Criteria}

The data samples described in Section 5.1 have first to pass the common data quality certified by the DØ DQ group [101]. The events belonging to bad runs or LBNs caused by detector operation failures or bad luminosity measurements are discarded from further use in the analysis. Note that as pointed in Section 5.1.1, the total integrated luminosity numbers in this analysis $2.64 \mathrm{fb}^{-1}$ and $2.78 \mathrm{fb}^{-1}$ are scaled down by $3 \%$, due to inefficient rejection related to calorimeter noise of data quality. A combination of different triggers, also discussed in Section 5.1.1, are employed to further reject un-related background events in data samples. The simulated events are reweighted with the corresponding trigger efficiencies.

Every event must have its primary vertex reconstructed in the effective hard scattering interaction region $|z|<60 \mathrm{~cm}$. There must be at least three tracks attached to the primary vertex. The passed events are considered for identifying the $\mathrm{W}$ and Higgs bosons.

\subsection{1 $W$ Boson Identification}

The $W$ bosons in this analysis are identified through its leptonically decay $W \rightarrow e \nu$ and $W \rightarrow \mu \nu$. The final state particles must contain exclusively one isolated energetic electron or muon and significant missing $E_{T}$.

\section{Electron Selection}

Electron candidates in an event are first required to pass the basic criteria dis-

cussed in Section 4.1.2, as the following: $f_{E M}=\frac{E_{E M}(0.2)}{E_{\text {total }}(0.4)}>0.9, f_{\text {iso }}=\frac{E_{\text {total }}(0.4)-E_{E M}(0.2)}{E_{E M}(0.2)}<$ 0.15, matched to central tracks, $\chi_{H M x 7}^{2}<50$ and electron likelihood $>0.85$.

The electrons reconstructed in central calorimeter (CC) $|\eta|<1.1$ and endcap calorimeter (EC) $1.5<|\eta|<2.5$ are used in the analysis. The transverse momenta 
of the electrons must satisfy $p_{T}>15 \mathrm{GeV}$. To reduce the background events with two or more electrons in the final states such as those from $Z$ and $t \bar{t}$ production, there must be exactly one electron passing the above selection criteria. If there is any second electron satisfying all criteria (without likelihood requirement) but failing either $p_{T}>15 \mathrm{GeV}$ or $|\eta|<3.0$, the event will also be rejected.

An example of the kinematic distributions of the selected electrons is shown in Fig. 5.1. Note that events shown in the plots have passed all the kinematic event selection criteria and properly normalized with correction factors, part of which are discussed in the following sections.

\section{Muon Selection}

The basic selection criteria for muons are explained in Section 4.1.5, summarized as the following: (1) medium nseg $=+3$ muon, which rejects $\sim 10 \%$ of high $p_{T}$ muons, mainly in the detector bottom region where no full coverage of $\mathrm{A}$ and $\mathrm{BC}$ layer is availabe. (2) medium track with the central track fit $\chi^{2} / n d f<4$ to remove bad tracks. (3) To reject cosmic muons, scintillator hitting time of A and BC layers has to satisfy $\left|t_{A}\right|<10$ ns and $\left|t_{B C}\right|<10$ ns.

The muon isolation information is used to discriminate the muons of $W$ decay from the muons produced in jet decays. The separation of a muon from the nearest reconstructed jets is required to be $\Delta R(\mu$,jet $)>0.5$. Two additional variables in Section 4.1 .5 are used to further reject muons originated from heavy flavor jets by requiring ScaleCalorimeter Halo $<0.08$ and ScaledTrackHalo $<0.06$.

The muons must be reconstructed with $|\eta|<2.0$ and $p_{T}>15 \mathrm{GeV}$. Events with two or more muons satisfying the above selection without ScaleCalorimeter Halo < 0.08 and ScaledTrackHalo $<0.06$ requirement are discarded. An example of the muon kinematic distribution is shown in Fig. 5.2. 


\section{$\mathbb{E}_{T}$ Selection and Transverse Mass of $W$ Boson}

The missing $E_{T}$, approximating the neutrino transverse energy has to pass the requirement of $\mathbb{E}_{T}>20 \mathrm{GeV}$ to select the $\mathrm{W}$ boson. The transverse mass of $W$ boson can be reconstructed from the measured kinematic variables of lepton and $\mathbb{E}_{T}$,

as $m_{T}^{W}=\sqrt{E_{T}^{2}-\vec{p}_{T}^{2}}=\sqrt{2 p_{T}^{l e p} E_{T}\left(1-\cos \left(\phi_{l e p}-\phi_{m e t}\right)\right)}$. The distributions of $E_{T}$ and $W$ transverse mass for the selected events are shown in Fig. 5.2.1.

\subsubsection{Jet Selection}

As discussed in Section 4.1.3, the jets used in this analysis are reconstructed with the $R=0.5$ cone algorithm. A set of common identification cuts, also explained in Section 4.1.3, are employed to select well reconstructed jets: (1) $5 \%<E M F<95 \%$. (2) $C H F<0.4$. (3) HOTF $<10$. (4) $n 90>1$. (5) L1Conf $>0.4$ in CC and EC, L1Conf $>0.2$ in ICD.

The jets reconstructed within the detector region $|\eta|<2.5$ with $p_{T}>20 \mathrm{GeV}$ are considered in the analysis. In addition, further kinematic cuts are explored to optimize the results. The jet with the highest $p_{T}$ in an event is required to be $p_{T}>25 \mathrm{GeV} . H_{T}$, sum of the $p_{T}$ of all jets in an event, must be larger than $60 \mathrm{GeV}$ to reduce the effects of low energy jets. The events with 2 or 3 jets satisfying the above requirements are selected and treated separately in this analysis.

The invariant mass of the two leading $p_{T}$ jets as well as their angular separations are reconstructed in the analysis. They are used as the main variables to discriminate the Higgs signal events from background events, to be discussed later. The distributions of the 2-jet selected events are shown in Fig. 5.4. 


\subsubsection{Multijet Background Estimation}

The multijet (QCD) background events are estimated from data samples since full simulation of these complicated processes is not available. In the case of muons, it is possible that muons originating from jets could pass the muon selection of this analysis and be misidentified as $W$ boson events. These background events are estimated via the so-called Matrix Method [102] using samples containing loose and tight muon events. The tight muon criteria means that muon passes all the event selection, while the loose applies all the event selection criteria but not the ScaleCalorimeter Halo and ScaledTrackHalo requirements. The number of events follows the equations

$$
\begin{aligned}
& N_{\text {loose }}=N_{W}+N_{Q C D} \\
& N_{\text {tight }}=\epsilon_{W} N_{W}+\epsilon_{Q C D} N_{Q C D}
\end{aligned}
$$

where $N_{\text {loose }}$ and $N_{\text {tight }}$ represent the total numbers of events passing the loose and tight muon selection criteria, $N_{W}$ and $N_{Q C D}$ are the number of real $W$ and multijet events respectively. $\epsilon_{Q C D}$ is the probability for a multijet event in loose muon sample to pass the tight muon criteria, while $\epsilon_{W}$ is the probability for the real $W$ boson event.

The equations can be inversely solved to be

$$
\begin{aligned}
N_{W} & =\frac{N_{\text {tight }}-\epsilon_{Q C D} N_{\text {loose }}}{\epsilon_{W}-\epsilon_{Q C D}} \\
N_{Q C D} & =\frac{\epsilon_{W} N_{\text {loose }}-N_{\text {tight }}}{\epsilon_{W}-\epsilon_{Q C D}} .
\end{aligned}
$$

$\epsilon_{W}$ in this analysis is approximated by counting the ratios of simulated loose muon $W$ events passing the tight muon selection. $\epsilon_{Q C D}$ is calculated by using data events satisfying all the event selection criteria but $\mathscr{H}_{T}$ is required to be $\mathbb{E}_{T}<10 \mathrm{GeV}$. The passing ratio of loose muon events to tight muon events, denoted as $\epsilon_{Q C D}$, is parameterized as a function of muon $p_{T}$. The real $W$ events with $\mathbb{E}_{T}<10 \mathrm{GeV}$ 


\begin{tabular}{|c|cc|cc|}
\hline Trigger List & Fake Rate & 2 jets (\%) & Fake Rate & 3 jets (\%) \\
& CC & EC & CC & EC \\
\hline \hline v8-11 & $5.4 \pm 0.2$ & $6.3 \pm 0.2$ & $6.0 \pm 0.2$ & $7.7 \pm 0.2$ \\
v12 & $5.7 \pm 0.1$ & $8.2 \pm 0.1$ & $8.4 \pm 1.0$ & $8.3 \pm 0.3$ \\
v13 & $6.0 \pm 0.1$ & $8.5 \pm 0.1$ & $8.2 \pm 1.0$ & $10.3 \pm 1.0$ \\
v14 & $6.6 \pm 0.1$ & $8.8 \pm 0.1$ & $6.6 \pm 1.0$ & $8.8 \pm 1.0$ \\
v15-16 & $6.6 \pm 0.1$ & $8.8 \pm 0.1$ & $9.6 \pm 1.0$ & $11.6 \pm 1.0$ \\
\hline
\end{tabular}

Table 5.13: Fake rate for $\mathrm{CC}$ and EC electron channel at $\mathrm{pT}=30 \mathrm{GeV}$ for $\mathrm{v} 8$-v15 trigger lists. Errors are statistical only. An additional $15 \%$ systematic errors on these parameters is considered when estimating the total uncertainty on the QCD background.

are also taken into account by subtracting simulated $W$ event contributions from the data events in this calculation. The numbers of real $W$ events and multijet events passing all the event selection, $\epsilon_{W} N_{W}$ and $\epsilon_{Q C D} N_{Q C D}$, are estimated using the resulted $\epsilon_{W}$ and $\epsilon_{Q C D}$, as shown in Fig. 5.5.

In the case of electrons, a jet from the multijet background processes could be misidentified as an electron. The estimation procedures of multijet background are basically the same, except that $\epsilon_{Q C D}$ has various dependences on electron $p_{T}$, $\Delta \phi\left(e, \mathbb{E}_{T}\right)$, jet multiplicity and trigger requirements. The fake rates of $\epsilon_{Q C D}$ at electron $p_{T}=30 \mathrm{GeV}$ are summarized in Tab. 5.13. More details can be found in [103].

The multijet background estimation is limited in the low $\mathbb{E}_{T}$ and $m_{T}^{W}$ regions because of mismeasurement of $\mathscr{E}_{T}$. These background events tend to have the same $\mathbb{E}_{T}$ and lepton direction and low $m_{T}^{W}$. To further reject the multijet background, a triangular cut of $m_{T}^{W}>40-0.5 \mathbb{E}_{T}$ is applied in the cases of both muon and electron. As shown in Fig. 5.2.3, the triangular cut rejects most of the multijet events but reasonably keeps the $W H$ events. 


\subsubsection{Normalization of Simulated Events \\ Scale Factors}

The simulated events are normalized according to the integrated luminosity of data events and the predicted cross sections in the Standard Model, as listed in Section 5.1.2. Each generated event $i$ in a physics process is weighted by

$$
w_{i}=\frac{\sigma \times \mathcal{L}}{N_{g e n}}
$$

where $\sigma$ is predicted cross section of the physics process, $\mathcal{L}$ is the integrated luminosity of data events and $N_{g e n}$ is the total number of simulated events of the physics process generated for the analysis.

However, due to the large uncertainties of cross sections in the $W+$ jets and $Z+$ jets calculations, the number of events in $W / Z+$ jets samples are further scaled to ensure that simulated samples and data samples have the same number of events. The scale factors is defined as

$$
S F=\frac{N_{\text {data }}-N_{Q C D}-N_{S M}}{N_{W / Z+j e t s}}
$$

where $N_{\text {data }}, N_{Q C D}$ and $N_{S M}$ are the number of events in data, multijet and the simulated processes except for $W / Z+$ jets. $N_{W / Z+j e t s}$ is the total number of $W / Z+$ jets, where $W+$ jets and $Z+$ jets samples are simply added together. The values of $S F$ are summarized in Tab. 5.14. Note that they are calculated with all the event selection criteria except for the b-jet tagging selection, which is discussed in the next section.

For $W / Z+$ heavy flavor jets samples, additional scale factor called Heavy Flavor (HF) factor is applied. The values of HF factors are determined using orthogonal samples with b-jet tagging selection, as

$$
H F=1+\frac{N_{\text {data }}^{0 t a g}-N_{\text {expected }}^{0 \text { tag }}}{N_{W_{b b}+W_{c c}+Z_{b b}+Z_{c c}}^{0 t a g}}
$$




\begin{tabular}{|l|l||c|c|}
\hline & channel & 2 jet & 3 jet \\
\hline Run IIa & Electron (CC) & $1.65 \pm 0.01$ & $1.62 \pm 0.03$ \\
& Muon & $1.63 \pm 0.01$ & $1.80 \pm 0.03$ \\
\hline Run IIb & Electron (CC) & $1.85 \pm 0.01$ & $2.05 \pm 0.03$ \\
& Muon & $1.52 \pm 0.01$ & $1.73 \pm 0.03$ \\
\hline
\end{tabular}

Table 5.14: The scale factor $S F$ applied to $W / Z+j e t s$ samples. (errors are statistical only, the uncorrelated systematic uncertainties between the $e$ and $\mu$ determination is greater than $10 \%)$.

where $N_{\text {data }}^{0}, N_{\text {expected }}^{0 t a g}$ and $N_{W_{b b}+W_{c c}+Z_{b b}+Z_{c c}}^{0 t a g}$ are the total numbers of events with no b-tagged jets in their respective data samples, the expected standard model processes (including multijet background) and $W / Z+h . f . j e t s$ samples respectively. It is calculated iteratively with $S F$ factor applied. The values of the HF factors are summarized in Tab. 5.15. The VeryTight, Tight and oldLoose are b-jet tagging operating points which are explained in the next section.

\begin{tabular}{|c|l||ccc|}
\hline data & btag OP & VeryTight & Tight & oldLoose \\
\hline Run IIa & Electron & $0.99 \pm 0.02$ & $0.99 \pm 0.02$ & $0.97 \pm 0.03$ \\
& Muon & $1.02 \pm 0.02$ & $1.02 \pm 0.02$ & $1.01 \pm 0.03$ \\
Run IIb & Electron & $1.29 \pm 0.02$ & $1.36 \pm 0.02$ & $1.34 \pm 0.03$ \\
& Muon & $1.45 \pm 0.02$ & $1.52 \pm 0.02$ & $1.38 \pm 0.03$ \\
\hline
\end{tabular}

Table 5.15: The HF factor determined with b-jet tagging operation points VeryTight, Tight and oldLoose on different datasets. (errors are statistical only, the uncorrelated systematic uncertainties between the $e$ and $\mu$ determination is greater than $10 \%)$.

\section{$W / Z+j e t s$ Events Reweighting}

The deficiency of modeling in $A L P G E N$ for $W / Z+$ jets is taken into account by reweighting the angular distributions of jets in $W / Z+$ jets events to minimize the kinematic shape difference between data and simulated events. To extract the reweighting function, the data events are first subtracted by other Standard Model background processes and then divided by the $W / Z+j e t s$ events. The 4 angular distributions reweighted are $\eta$ of the leading two jets, $\Delta \eta$ and $\Delta \phi$ between the leading two jets. The reweighting functions are shown in Fig. 5.7. The specific uncertainty 
due to jet reweighting is $5 \%$ as shown shown in Fig. 5.8 .

Impacts of Event Generators on $W H$ samples

The $W H$ event samples in this analysis is generated using PYTHIA, which calculates with LO matrix elements and performs parton showering and hadronization. The cross sections of $W H$ used for normalization are scaled to NNLO cross sections by multiplying $\mathrm{K}$ factors. Some other event generators such as $M C @ N L O$ calculates with NLO corrections and use HERWIG for parton showering and hadronization. The potential effects due to different event generators are studied on $W H$ samples by comparing the events generated by PYTHIA, MC@NLO and HERWIG. No obvious difference is observed except the transverse momenta of b quarks at parton level. However, the effects of b quark difference are not visible in the reconstructed jets [104]. A conservative uncertainty of $5 \%$ is thus assigned to the acceptance of $W H$ events.

\section{Contributions from $W \gamma^{*}$ Events}

For the diboson background processes, the $W \gamma^{*}$ process is not implemented, but only $W Z$ is available in PYTHIA. To estimate the contributions of $W \gamma^{*}$ events, $W Z / \gamma^{*}$ events are generated using $H E R W I G$ and are compared to $W Z$ generated by PYTHIA. No significant difference is observed after the event selections. The number of events varies less than 1\% [104]. The possible contributions from $W \gamma^{*}$ events are thus ignored.

\subsection{The Event Selection of b-jet Tagging}

Once the events are selected and corrected as discussed in the previous sections, the DØ NN b-jet tagger is used to identify b jets. Starting with a loose operating point, if two jets in an event are both tagged as loose b jets, the event is selected 
into the double tagged (DT) sample. Otherwise, a tight operation point is further used to tag the jets in this event. If one of the jets gets tagged as a b jet, this event will be selected to the single tagged (ST) sample. If neither applies, the event is labeled as 0tag, which is used for determining the HF factor in Section 5.2.4. The orthogonal ST and DT event samples are combined to improve the sensitivity to the Higgs signal.

oldLoose and Tight as described in Section 4.3.1 are chosen as the loose and tight tagging points respectively. The corresponding b-tagging efficiencies are $\sim 60 \%$ for oldLoose with a fake rate of $\sim 1.5 \%$, and $\sim 50 \%$ for Tight with a fake rate of $\sim 0.5 \%$. The jet taggability is typically $\sim 80 \%$ in a two jet event samples with uncertainty of $3 \%$ per jet.

The kinematic distributions of data events are compared to the sum of the background events. As shown in Fig. 5.9, in the distributions of the leading jet $p_{T}$, the second leading jet $p_{T}$, the $\Delta R$ and invariant mass of the two leading jets in the $W+2 j e t s$ sample with exactly one jet b-tagged as Tight, data events are consistent with the simulated events. The $W+$ light jets and multijet events are still overwhelming the background. The kinematic distributions of $W+2 j$ ets with two jet b-tagged as oldLoose are shown in Fig. 5.10. The dijet mass distributions with single and double b-tagged jets of $W+2 / 3$ jet events are shown in Fig. 5.11 (linear scale) and Fig. 5.12 (logarithmic scale). The background events consist of mainly $W+b \bar{b}$ events and $t \bar{t}$ events. The total number of events in the data are well described by simulated events, as summarized in Tab. 5.16. 


\begin{tabular}{|c|c|c|c|c|c|c|c|c|c|c|c|c|c|c|c|c|c|c|}
\hline & $W$ & + & 2 jets & $\begin{array}{l}W \\
(1\end{array}$ & $\begin{array}{l}+ \\
b\end{array}$ & $\begin{array}{l}2 \text { jets } \\
\text { tag) }\end{array}$ & $\begin{array}{l}W \\
(2\end{array}$ & $\begin{array}{l}+ \\
b\end{array}$ & $\begin{array}{l}2 \text { jets } \\
\text { tag) }\end{array}$ & $W$ & + & 3 jets & $\begin{array}{l}W \\
(1\end{array}$ & $\begin{array}{l}+ \\
b\end{array}$ & $\begin{array}{l}3 \text { jets } \\
\text { tag) }\end{array}$ & $\begin{array}{l}W \\
(2\end{array}$ & $\begin{array}{l}+ \\
b\end{array}$ & $\begin{array}{l}3 \text { jets } \\
\text { tag) }\end{array}$ \\
\hline$W H, Z H$ & 15.8 & \pm & 2.7 & 6.8 & \pm & 1.3 & 3.9 & \pm & 0.7 & 3.8 & \pm & 0.6 & 1.6 & \pm & 0.3 & 1.0 & \pm & 0.2 \\
\hline$W W, W Z, Z Z$ & 1453 & \pm & 244 & 87 & \pm & 16 & 13.7 & \pm & 2.6 & 302 & \pm & 51 & 23.1 & \pm & 4.2 & 3.9 & \pm & 0.7 \\
\hline$W / Z+b \bar{b}$ & 1769 & \pm & 353 & 592 & \pm & 109 & 138 & \pm & 27 & 471 & \pm & 94 & 174 & \pm & 32 & 49.2 & \pm & 9.4 \\
\hline$t \bar{t}$ & 581 & \pm & 98 & 242 & \pm & 45 & 96.9 & \pm & 19 & 926 & \pm & 155 & 394 & \pm & 73 & 211 & \pm & 41 \\
\hline Single top & 290 & \pm & 49 & 123 & \pm & 23 & 31.5 & \pm & 6.1 & 91 & \pm & 15 & 38.5 & \pm & 7.0 & 16.9 & \pm & 3.2 \\
\hline Multijet & 3575 & \pm & 629 & 189 & \pm & 38 & 16.7 & \pm & 4.0 & 1228 & \pm & 216 & 92.8 & \pm & 18 & 14.4 & \pm & 3.4 \\
\hline$W / Z+$ jets & 44464 & \pm & 570 & 942 & \pm & 226 & 44.5 & \pm & 9.8 & 8357 & \pm & 105 & 239 & \pm & 57 & 25.7 & \pm & 5.8 \\
\hline $\begin{array}{l}\text { Total expectation } \\
\text { Observed Events }\end{array}$ & $\begin{array}{l}52148 \\
52148\end{array}$ & & (n.t.d.) & $\begin{array}{l}2182 \\
2174\end{array}$ & \pm & 348 & $\begin{array}{l}345 \\
336\end{array}$ & \pm & 51 & $\begin{array}{l}11379 \\
11379\end{array}$ & & (n.t.d.) & $\begin{array}{l}963 \\
912\end{array}$ & \pm & 152 & $\begin{array}{l}322 \\
321\end{array}$ & \pm & 49 \\
\hline
\end{tabular}

Table 5.16:

Summary table for the $W+2,3$ jet final states. Observed events in data are compared to the expected number of $W+$ jet events before tagging, with exactly one tight $b$-tagged jet, and with exactly 2 loose $b$-tagged jets. First three columns are for the $W+2$ jet channel, the last three columns for the $W+3$ jet channel. Expectation originates from the simulation of $W H$ and $Z H$ (with $m_{H}=115 \mathrm{GeV}$ ), dibosons $(W W, W Z, Z Z$, labeled $W Z$ in the table), $W b \bar{b}$ production, top production ( $t \bar{t}$ and single-top), multijet background and " $W+$ jet" production, which contains light and $c$ quarks. All $Z$ processes are fully simulated, and included in the corresponding $W$ categories. The processes $W(Z) b \bar{b}$ and $W(Z)+$ light and/or $c$ jets are counted separately. "n.t.d." stands for "normalized to data". The uncertainties given include statistics and systematics. 

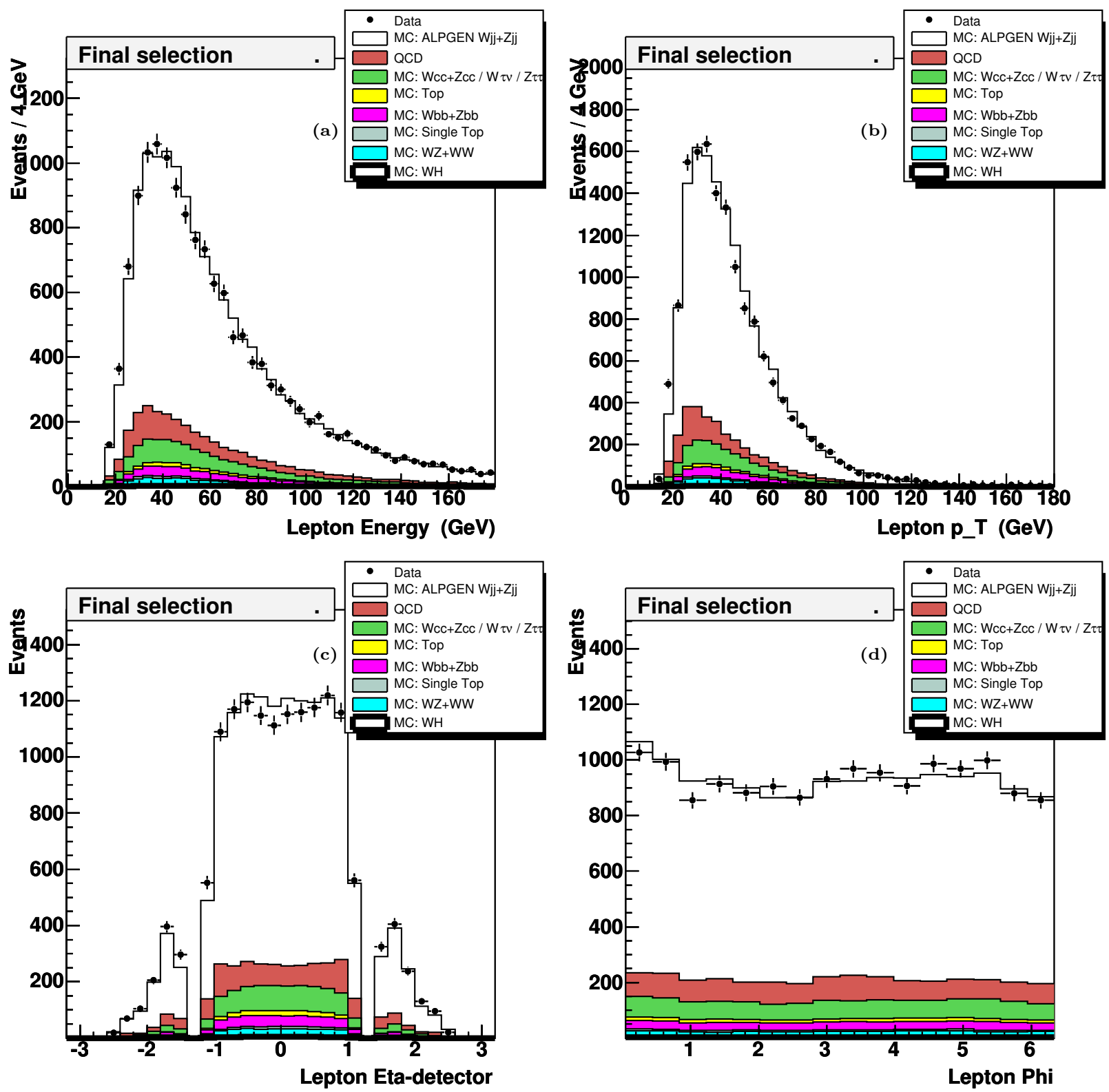

Figure 5.1: Kinematic distributions for CC+EC electrons of the $W+2$ jet event sample (Run IIb data set): a) energy; b) transverse momentum; c) $\eta$; d) $\varphi$. The simulation is normalized to the integrated luminosity of the data sample using the expected cross sections (absolute normalization) except for the $W+$ jets sample which is normalized to data on the "pre-tag sample", taking into account all the other backgrounds. 

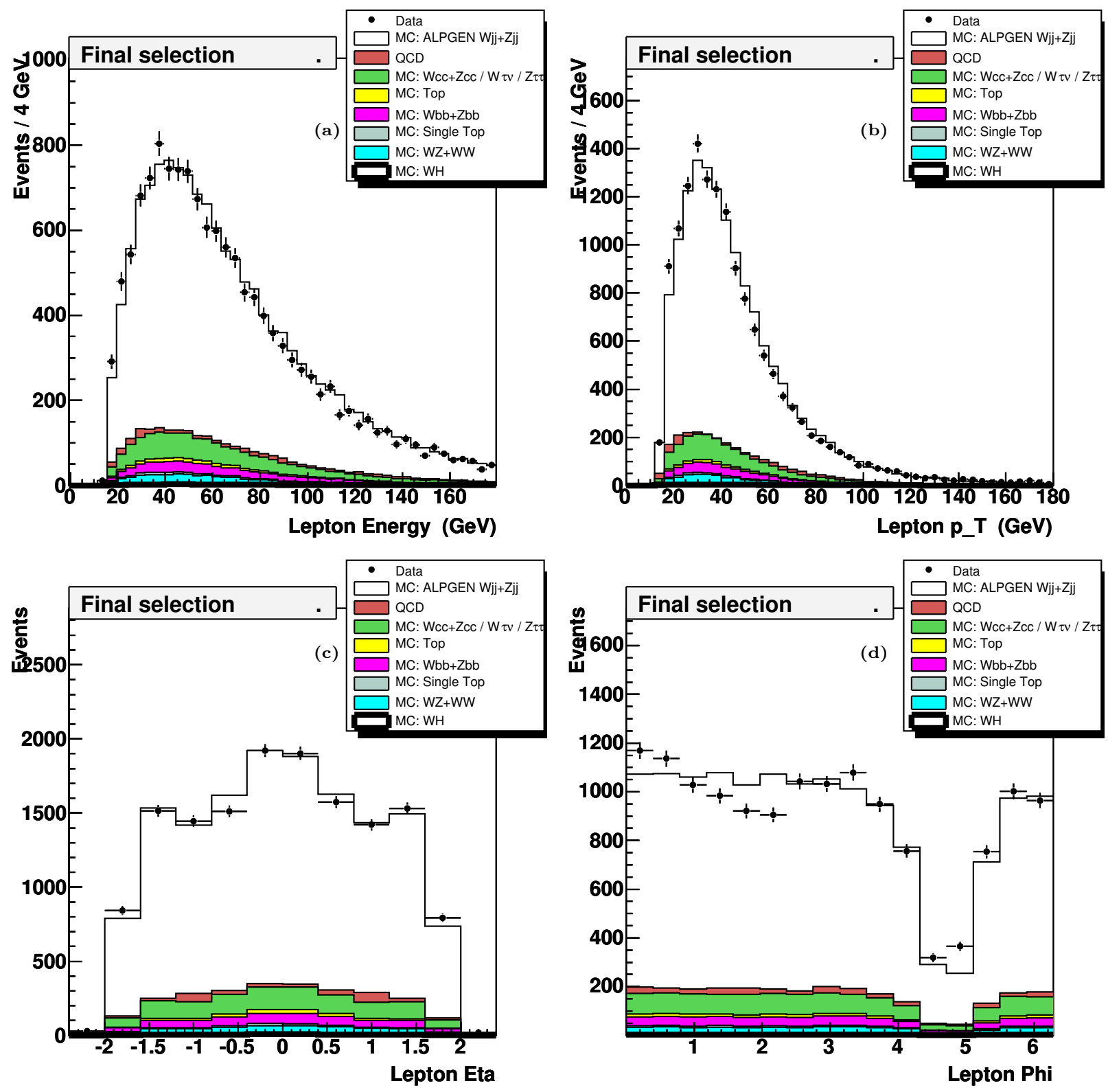

Figure 5.2: Kinematic distributions for muons of the $W+2$ jet event sample (Run IIb data set): a) energy; b) transverse momentum; c) $\eta$; d) $\varphi$. The simulation is normalized to the integrated luminosity of the data sample using the expected cross sections (absolute normalization) except for the $W+$ jets sample which is normalized to data on the "pretag sample", taking into account all the other backgrounds. 

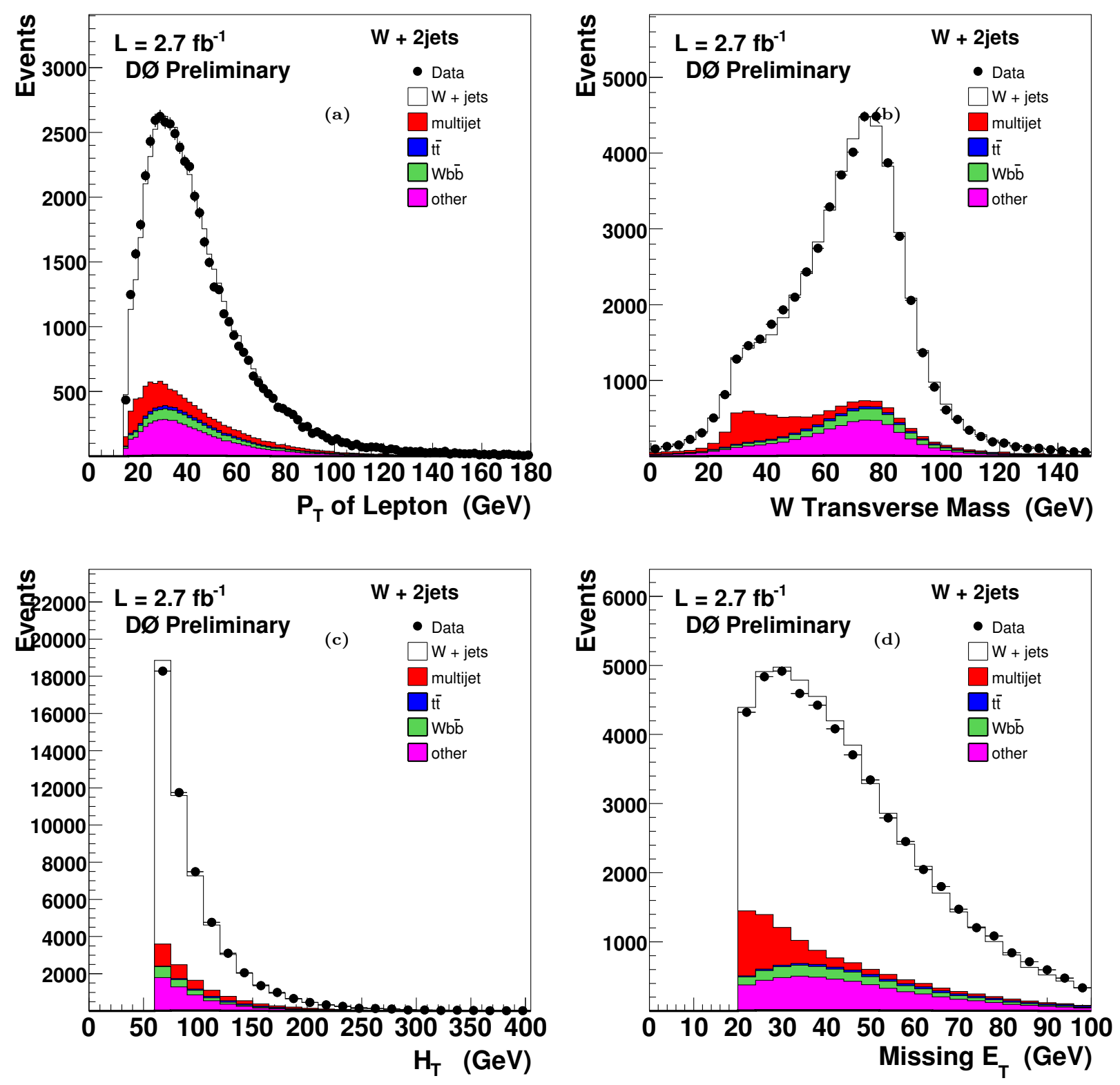

Figure 5.3: Distribution in the $W+2$ jets sample of the (a) lepton momentum, (b) the transverse $W$ mass, (c) the $H_{T}$ variable and (d) missing transverse energy compared to the simulated expectation in the $W+2$ jet event sample. The simulation is normalized to the integrated luminosity of the data sample using the expected cross sections (absolute normalization) except for the $W+$ jets sample which is normalized on the "untagged sample" to the data, taking into account all the other backgrounds. 

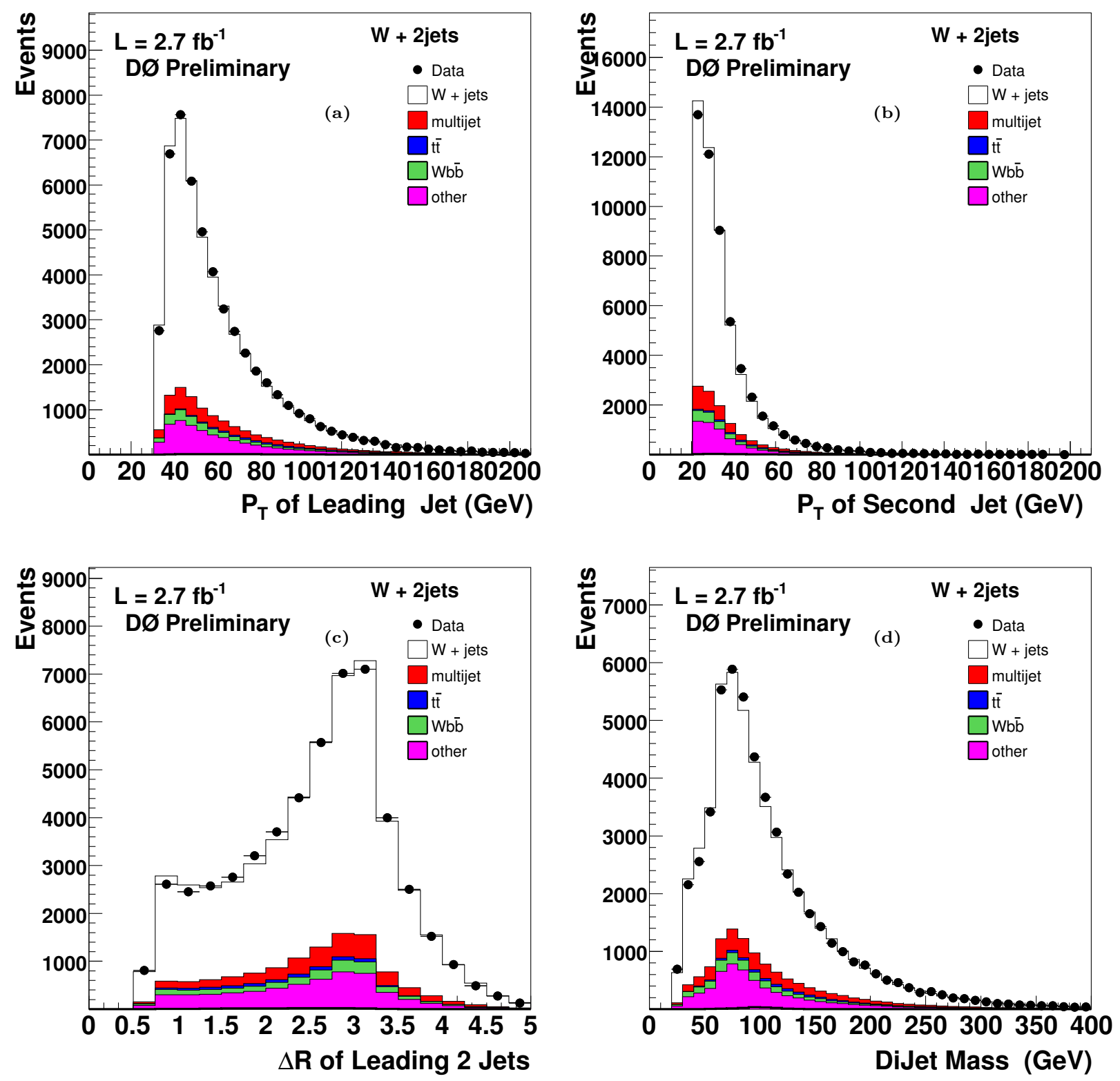

Figure 5.4: Distribution in the $W+2$ jets sample of the (a) $p_{T}$ of the leading and (b) next to leading jet, (c) of the distance in the $\eta-\varphi$ plane between the two jets and (d) of the dijet mass (d) between the two jets in the $W+2$ jet sample compared with the simulated expectation. The simulation is normalized to the integrated luminosity of the data sample using the expected cross sections (absolute normalization) except for the $W+$ jets sample which is normalized on the "untagged sample" to the data, taking into account all the other backgrounds. 


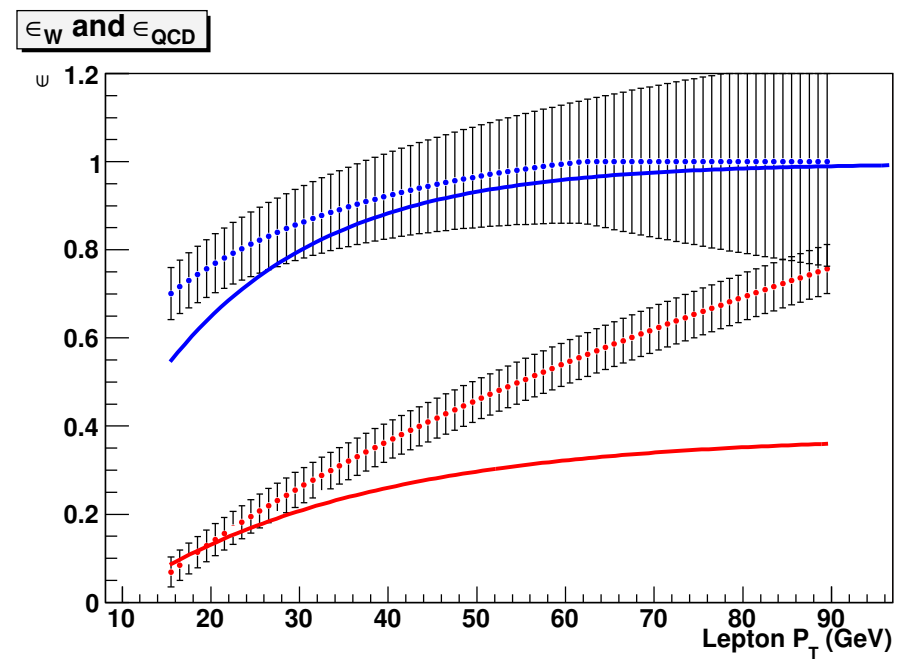

Figure 5.5: The $\epsilon_{W}$ and $\epsilon_{Q C D}$ as a function of muon $p_{T}$. The upper blue and lower red solid lines represent $\epsilon_{W}$ and $\epsilon_{Q C D}$ determined from Run IIb datasets, while the upper blue and lower red dotted lines are determined from Run IIa datasets. Note that the $W$ contributions to $\epsilon_{Q C D}$ is not subtracted in Run IIa calculations.
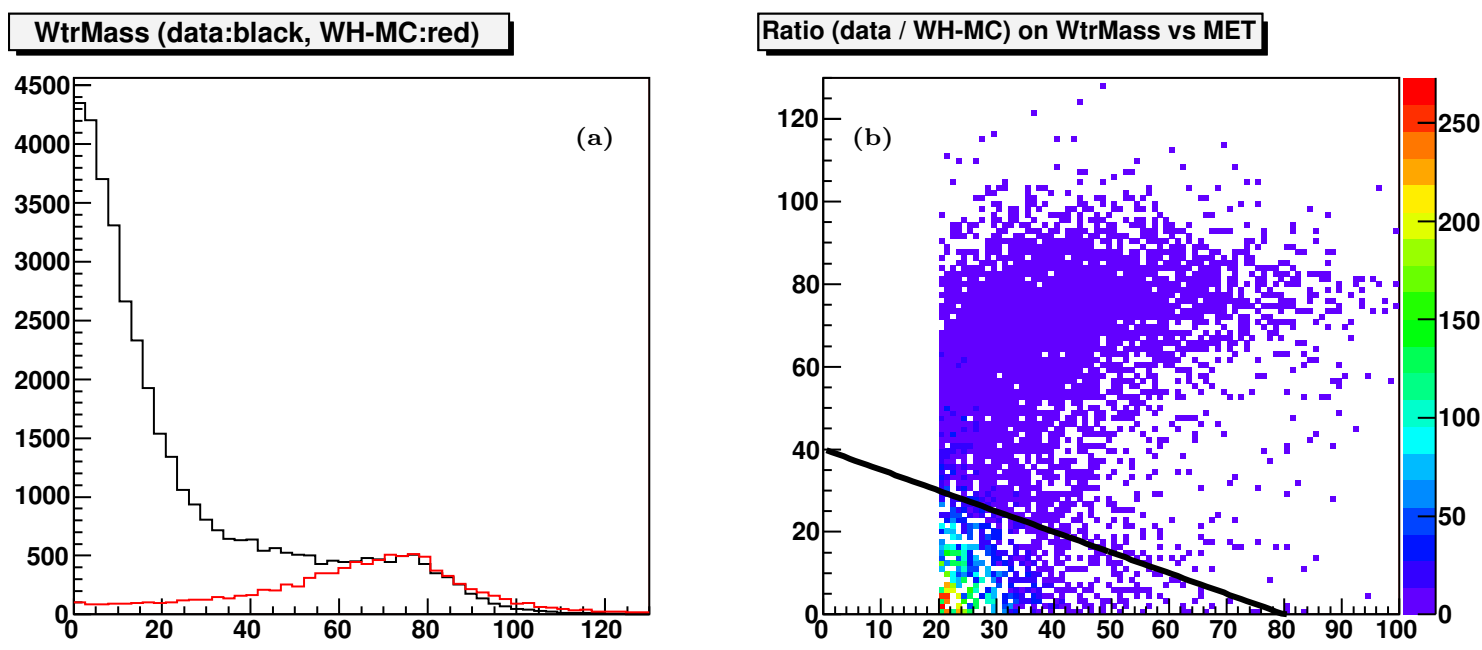

Figure 5.6: (a) W transverse mass for loose electron on data (black) as QCD sample and WH MC (red) as real W sample. (b) 2D ratio plot of data / WH MC on W transverse mass vs Missing Et. Normalization of WH MC is same as in Fig. (a). QCD events distribute low $E_{T}$ and low $M_{W}^{T}$ which can be eliminated by triangle cut of $M_{W}^{T}>-0.5 E_{T}+40 G e V$ as shown in black line. WH MC is normalized on W peak. 

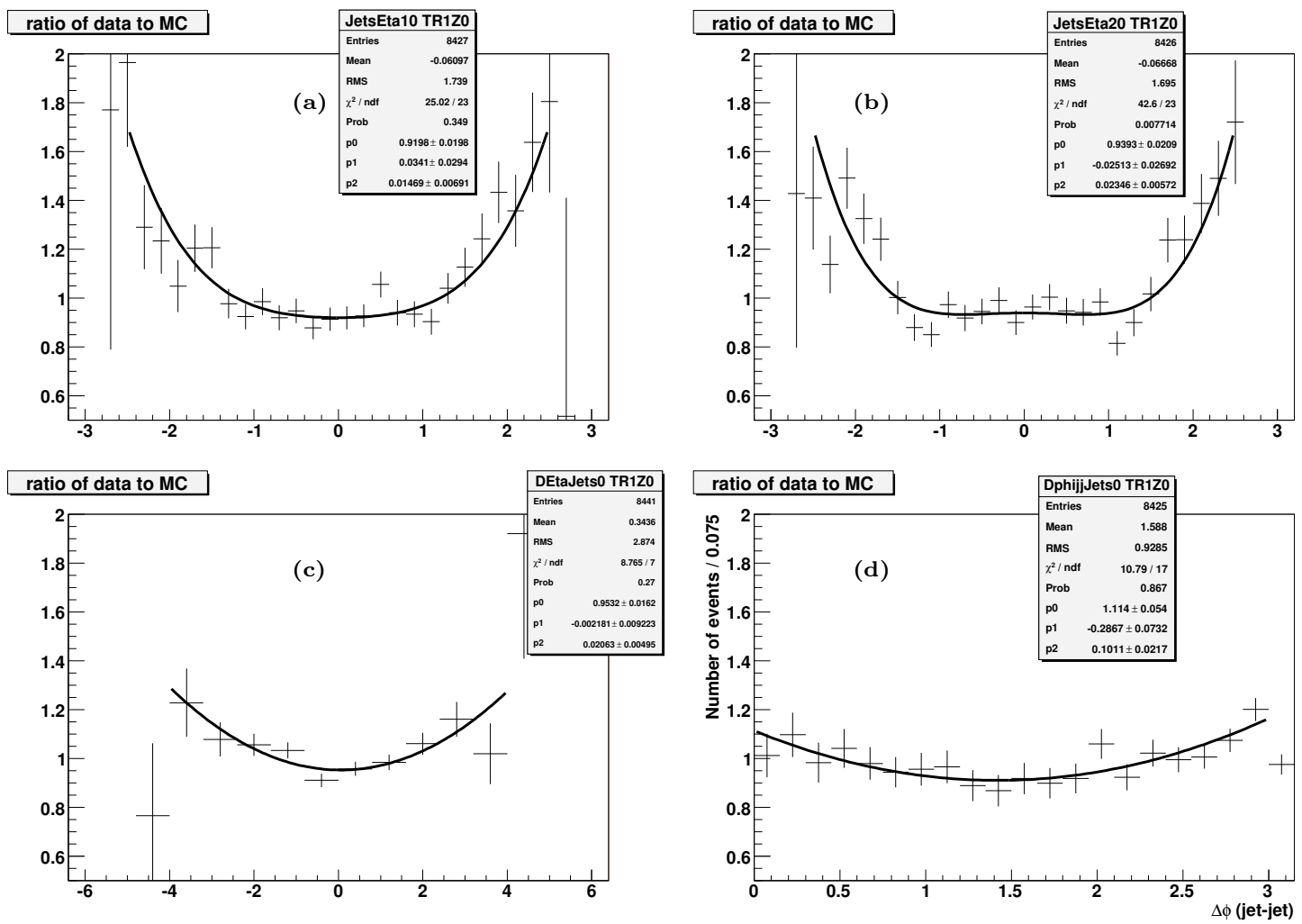

Figure 5.7: Reweighting functions on the jet angular distributions of simulated $W / Z+$ jets events. (a) Leading jet $\eta$. (b) $2^{n d}$ Leading jet $\eta$. (c) $\Delta \eta($ jet 1 , jet 2$)$. (d) $\Delta \phi($ jet 1 , jet 2$)$.

\section{Mass of di-Jets (Tight)}

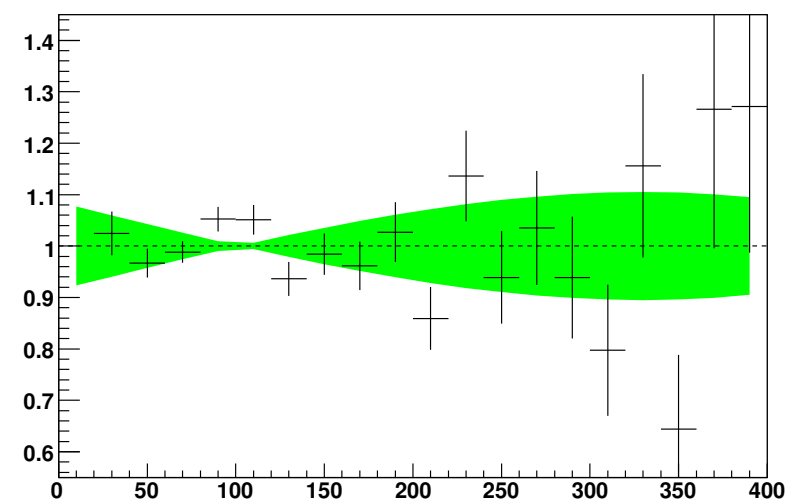

Mass of di-Jets (Tight)

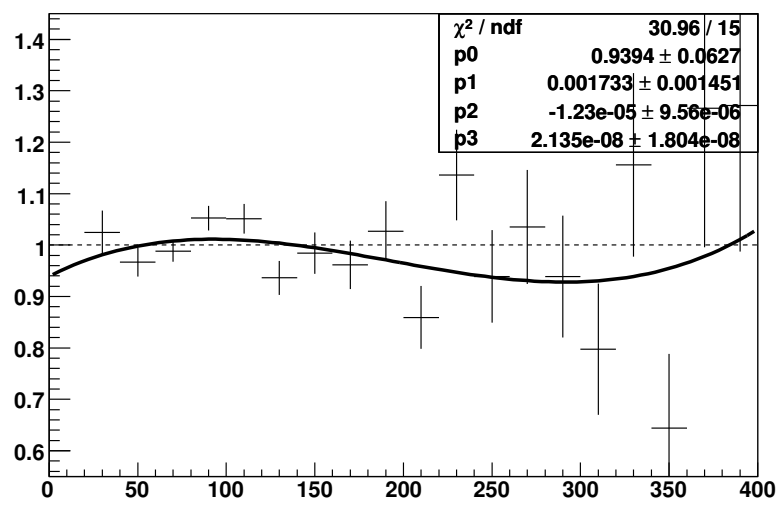

Figure 5.8: Shape uncertainty on the dijet mass in $W j j$ and $W b b$ events: a) uncertainty originating from the $\eta, \varphi, \delta-\eta, \delta-\varphi$ reweighting ; b) additional uncertainty on the shape as obtained by a fit to the ratio of data to the reweighted simulation to the data; the fitted result is also shown; the actual uncertainty is the fit to which is conservatively added another $+5 \%$ uncertainty. 

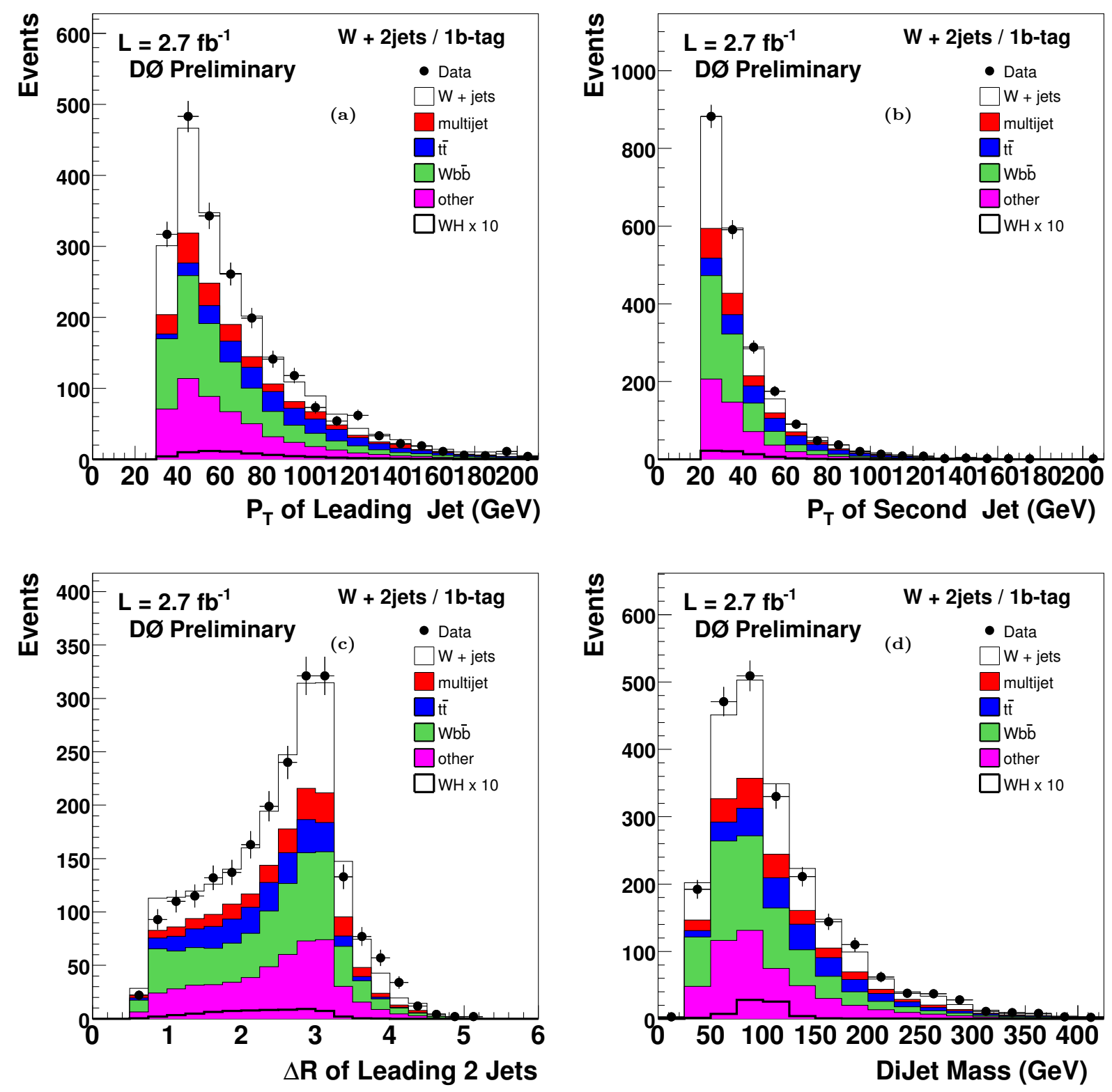

Figure 5.9: Kinematic distributions in the $W+2$ jets sample with exactly one jet b-tagged. (a) $p_{T}$ of the leading jet. (b) $p_{T}$ of the second leading jet. (c) $\Delta R$ between the two leading jets. (d) invariant mass of the leading two jets. The dots represent the distribution of data events, which are compared to the solid line representing the sum of the background events. The simulated events are fully normalized and corrected as discussed previous sections. The expected contribution of the Standard Model WH events with $m_{H}=115$ $\mathrm{GeV}$ shown in the plot scaled up by a factor of 10 . 

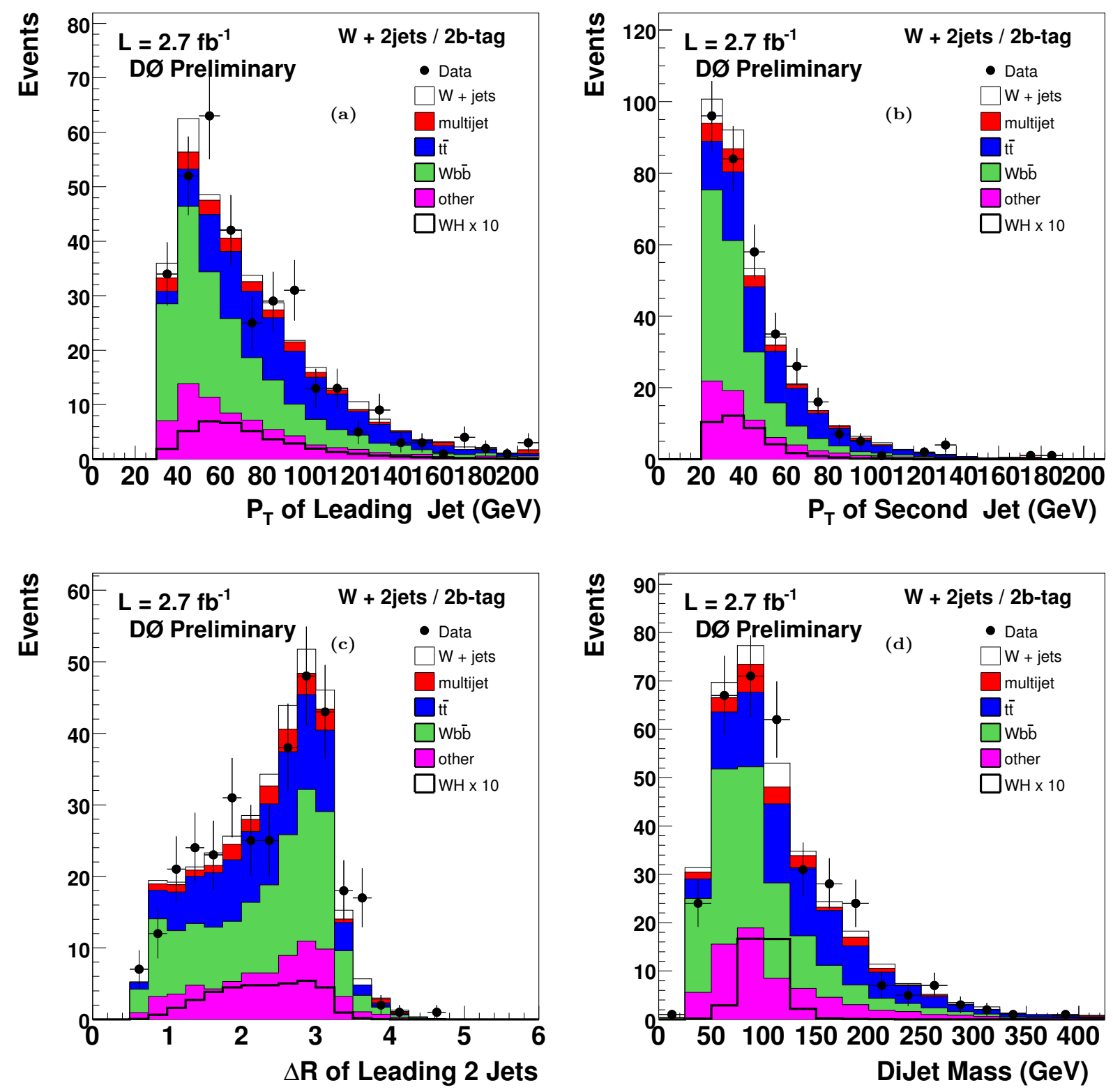

Figure 5.10: Kinematic distributions in the $W+2$ jets sample with 2 jet b-tagged. (a) $p_{T}$ of the leading jet. (b) $p_{T}$ of the second leading jet. (c) $\Delta R$ between the two leading jets. (d) invariant mass of the leading two jets. The dots represent the distribution of data events, which are compared to the solid line representing the sum of the background events. The simulated events are fully normalized and corrected as discussed previous sections. The expected contribution of the Standard Model WH events with $m_{H}=115$

GeV shown in the plot scaled up by a factor of 10 . 

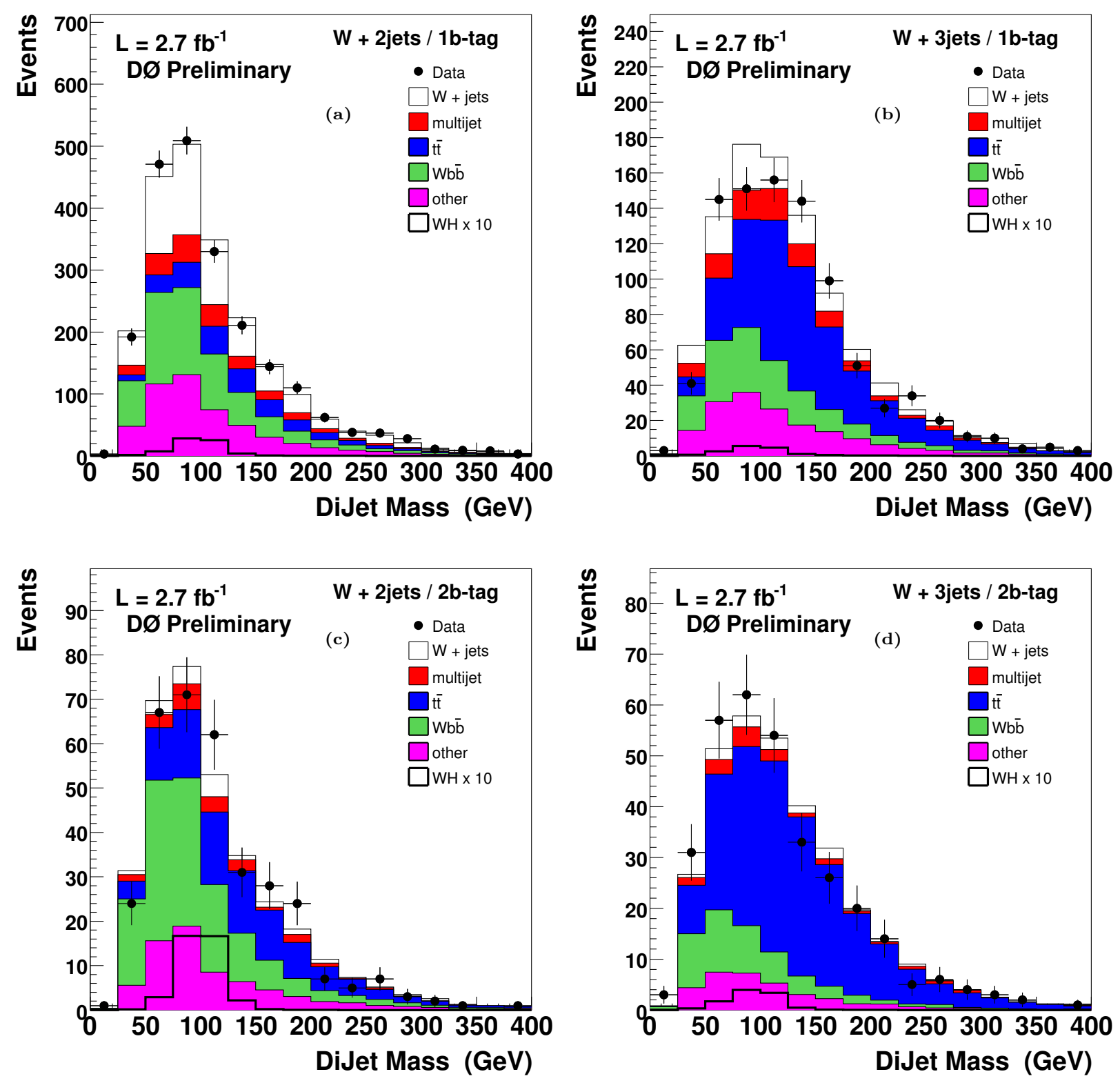

Figure 5.11: The dijet invariant mass distributions (linear scale) of events with single or double btagged jets. a) $W+2$ jet events with exactly one jet tagged as Tight $\mathrm{b}$ jet. b) $W+3$ jet events with exactly one jet tagged as Tight b jet. c) $W+2$ jet events with at least two jets tagged as oldLoose $\mathrm{b}$ jets. d) $W+3$ jet events with at least two jets tagged as oldLoose $b$ jets. The simulated processes are normalized to the integrated luminosity of the data sample using the expected cross sections (absolute normalization) except for the $W+$ jets sample which is normalized on the "untagged sample" to the data, taking into account all the other backgrounds. The backgrounds labeled as "other" in the figure are dominated by single-top production. Also shown is the contribution expected for standard model $W H$ production with $m_{H}=115 \mathrm{GeV}$, multiplied by a factor 10 . 

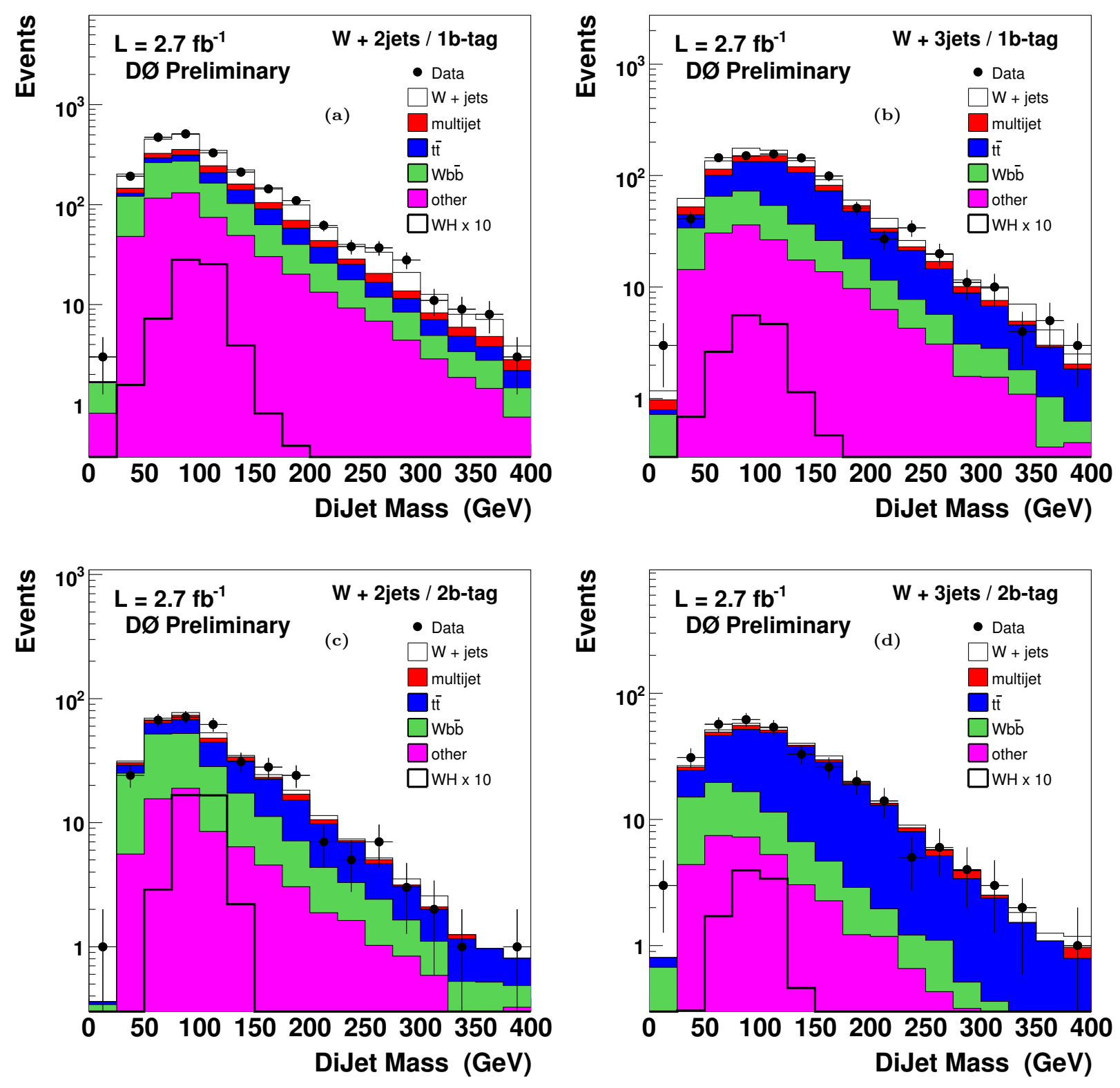

Figure 5.12: The dijet invariant mass distributions (logarithmic scale) of events with single or double b-tagged jets. a) $W+2$ jet events with exactly one jet tagged as Tight b jet. b) $W+3$ jet events with exactly one jet tagged as Tight b jet. c) $W+2 j e t$ events with at least two jets tagged as oldLoose $\mathrm{b}$ jets. d) $W+3$ jet events with at least two jets tagged as oldLoose $b$ jets. The simulated processes are normalized to the integrated luminosity of the data sample using the expected cross sections (absolute normalization) except for the $W+$ jets sample which is normalized on the "untagged sample" to the data, taking into account all the other backgrounds. The backgrounds labeled as "other" in the figure are dominated by single-top production. Also shown is the contribution expected for standard model $W H$ production with $m_{H}=115 \mathrm{GeV}$, multiplied by a factor 10 . 


\section{CHAPTER VI}

\section{Multivariate Analysis}

This analysis is challenged by the overwhelming background events, particularly the $W+b \bar{b}$ events which are irreducible to $W H$. Even when the optimized set of event selection criteria is applied, the signal significance $S / \sqrt{B}$ of $W+2$ jet events is $\sim 0.069$, which is increased to $\sim 0.146$ with one jet b-tagged and $\sim 0.210$ with two jet b-tagged. To maximize the sensitivity of this analysis, we explore two multivariate analysis techniques, the Matrix Element Method (ME) and Artificial Neural Network (ANN), to further discriminate the Higgs signal from background events. The description of the Matrix Element Method as well as the discriminant calculations is given in Section 6.1. The ME discriminant is used as one of the input variables to the Artificial Neural Network. The optimization of the neural network and its implementation details are summarized in Section 6.2.

\subsection{Matrix Element Method}

\subsubsection{Introduction to the Method}

Given that the set of reconstructed four-momenta of the physical objects in the final state is $\vec{x}$, the test on the hypothesis that an event is a signal event, is built as a Bayesian posterior probability

$$
P(S \mid \vec{x})=\frac{P_{S}(\vec{x})}{P_{S}(\vec{x})+P_{B}(\vec{x})}
$$


where $P(S \mid \vec{x})$ is the test for an event with final states $\vec{x}$ to be a signal event, $P_{S}(\vec{x})$ and $P_{B}(\vec{x})$ are the probability density functions for a signal and background event to appear in final states $\vec{x}$. The test is maximal when the set of variables $\vec{x}$ contains all the relevant information that distinguishes between signal and background events.

The Matrix Element Method defines event discriminant of signal against background as $D_{s}(\vec{x})=P(S \mid \vec{x})$ and makes use of all the available kinematic information in the event. The probability density functions can be numerically calculated based on the normalized differential cross sections of their corresponding physics processes. The differential cross sections are proportional to the parton-level matrix elements of the processes in the calculation, hence the name Matrix Element Method.

\subsubsection{Event Probability Density Function}

The event probability density function for a physics process is defined as the properly normalized differential cross section with reconstructed four-momenta $\vec{x}$,

$$
P(\vec{x})=\frac{1}{\sigma} \times \frac{d \sigma}{d \vec{x}}
$$

where $d \sigma(\vec{x})$ is the differential cross section at the reconstructed level, defined as

$$
d \sigma(\vec{x})=\sum_{i, j} \int d \vec{y}\left[f_{i}\left(q_{1}, Q^{2}\right) d q_{1} \times f_{j}\left(q_{2}, Q^{2}\right) d q_{2} \times \frac{d \sigma_{h s, i j}}{d \vec{y}} \times W(\vec{x}, \vec{y}) \times \Theta_{\text {parton }}(\vec{y})\right]
$$

with the terms explained below.

$\sum_{i, j}$ is the sum over the initial parton flavors in the hard scatter collision. For instance, the combinations of partons ij like $u \bar{d}, c \bar{s}, \bar{d} u$ or $\bar{s} c$ in $p \bar{p}$ collisions can produce $W^{+} H$ events via their annihilation.

$f_{i}\left(q_{1}, Q^{2}\right)$ and $f_{j}\left(q_{2}, Q^{2}\right)$ are the parton distribution functions of partons $i$ and $j$, which respectively carry momentum of $q_{1}$ and $q_{2}$. They are evaluated at the factorization scale $Q^{2}$ using leading-order PDFs of CTEQ6 [105] with LHAPDF [106] as the interface. 
$\frac{d \sigma_{h s, i j}}{d \vec{y}}$ is the differential cross section of the hard scatter collision as a function of four momenta $\vec{y}$ at parton level. It is proportional to the square of the matrix element, as

$$
d \sigma_{h s}=\frac{(2 \pi)^{4}}{4 \sqrt{\left(q_{1} q_{2}\right)^{2}-m_{1}^{2} m_{2}^{2}}}|\mathcal{M}|^{2} d \Phi_{n}(\vec{y})
$$

where the first term is the flux factor, $d \Phi_{n}(\vec{y})$ is the n-body phase space factor of which $n=4$ for two jet events, and $\mathcal{M}$ is the matrix element. In this analysis, the matrix elements are generated using MadGraph [107] at leading order. A total of 8 different matrix elements in the $2 \rightarrow 4$ processes are used, as shown in Fig. 6.1. For t-channel single top production, $t q$ is assigned as the final state for $2 \rightarrow 4$
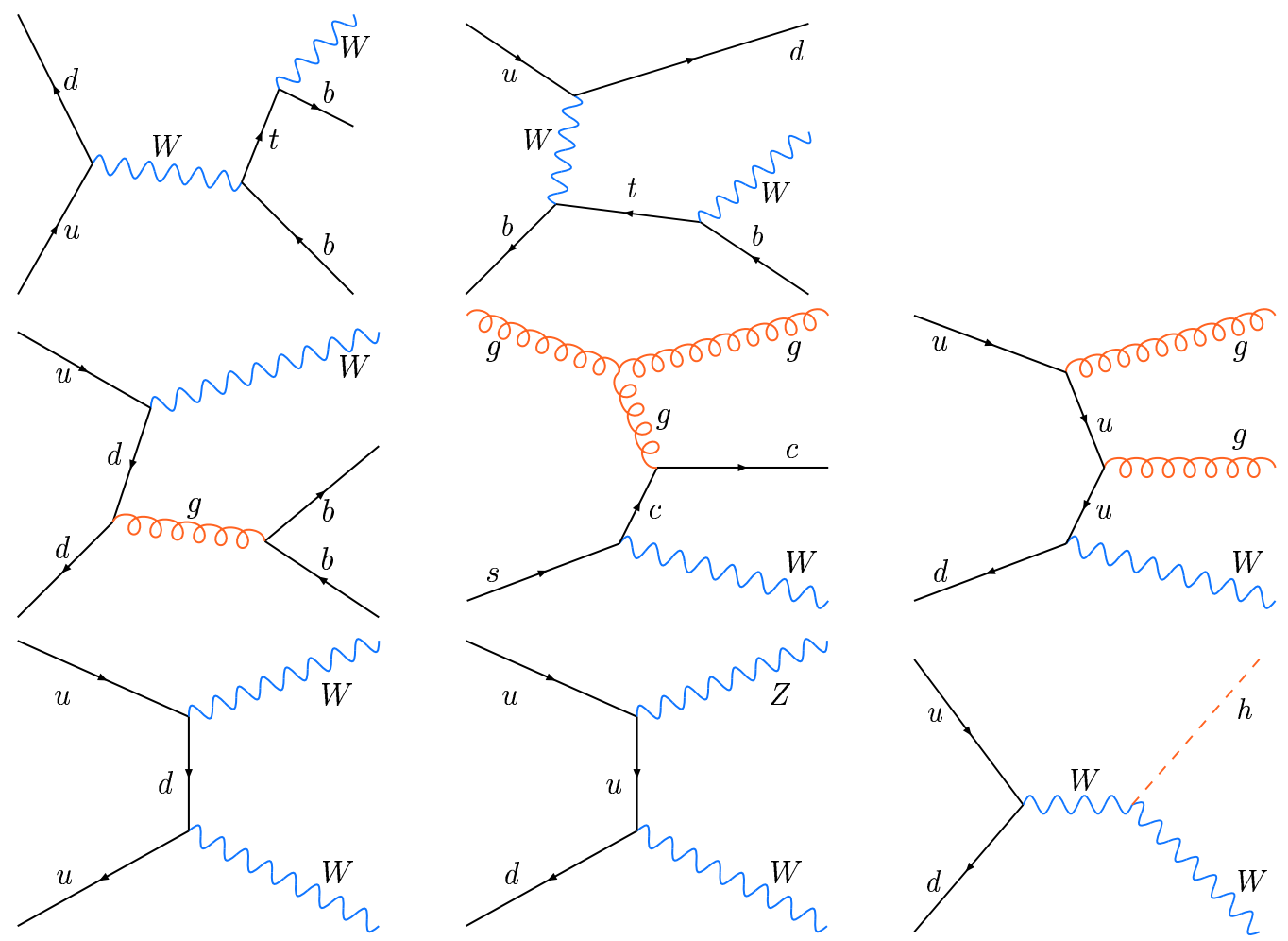

Figure 6.1: The corresponding Feynman diagrams of the leading-order matrix elements used in the calculation of event probability density functions. Upper row: $u d \rightarrow t b, u b \rightarrow t d$; Middle row: $u d \rightarrow W b b, s g \rightarrow W c g, u d \rightarrow W g g$. Lower row: $u u \rightarrow W W, u d \rightarrow W Z, u d \rightarrow W H$.

diagram. The processes $W g g, W c g$ and $W b b$ are chosen for $W+j e t s$ because they give the largest cross sections after applying the b-tagging efficiency when generated 
by MadGraph.

$W(\vec{x}, \vec{y})$ is the transfer function which stands for the conditional probability of the reconstructed states with $\vec{x}$ given the parton-level states $\vec{y}$. The transfer function is parameterized using simulated events for each object type such as jet, electron and muon, as well as for the different detector regions. The jet transfer functions [108] are considered to be the energy difference between the parton and the jet with angles well measured, as

$$
W_{\text {jet }}(\vec{x}, \vec{y})=W\left(E_{\text {jet }}-E_{\text {parton }}\right) \times \delta\left(\Omega_{\text {jet }}-\Omega_{\text {parton }}\right)
$$

where $W\left(E_{\text {jet }}-E_{\text {parton }}\right)$ are parameterized as double Gaussian functions. An example of the energy difference for light jets, b jets and soft-muon-tagged b jets is shown in Fig. 6.2. The electron transfer function [109] is assumed to be a function of

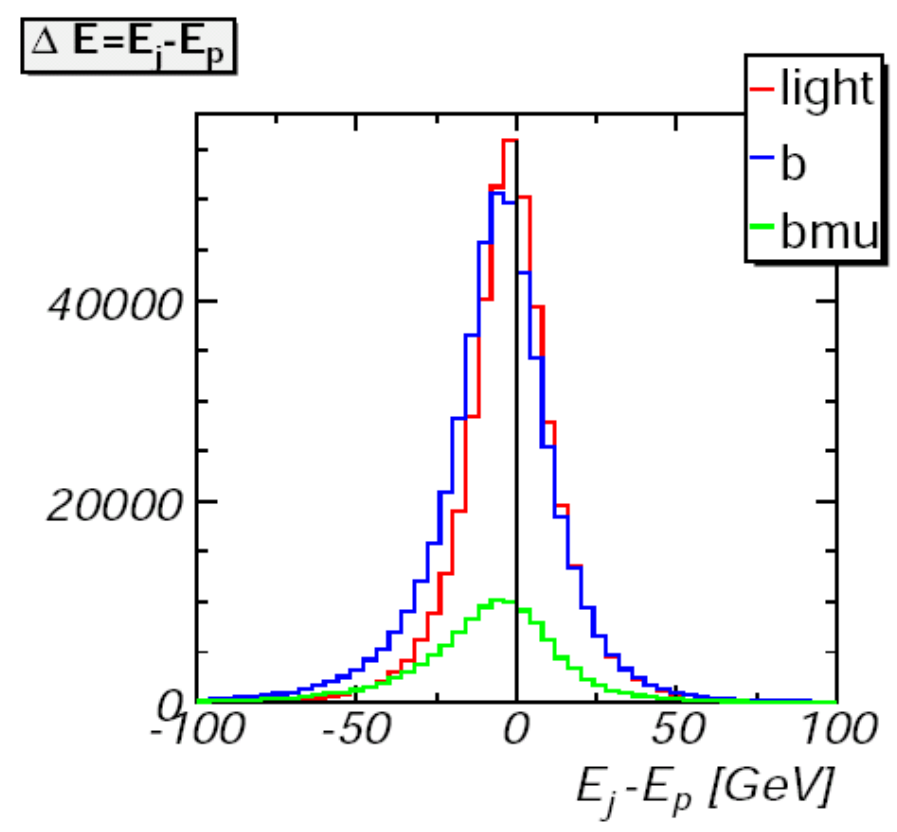

Figure 6.2: Energy difference between a reconstructed jet and its matched parton for three types of jets for all detector regions and all jet energies.

reconstructed electron energy $E_{e}$, parton-level electron energy $E_{p}$ and production 
angle $\theta$ with respect to the beam axis, as

$$
W_{\text {electron }}(\vec{x}, \vec{y})=W\left(E_{e}, E_{p}, \theta\right) \times \delta\left(\Omega_{e}-\Omega_{p}\right)
$$

where $W\left(E_{e}, E_{p}, \theta\right)$ is a Gaussian function with its width dependent on the electron energy. The muon transfer function [110] [111] is determined as functions of $\Delta\left(\frac{q}{p_{T}}\right)=$ $\left(\frac{q}{p_{T}}\right)_{\mu}-\left(\frac{q}{p_{T}}\right)_{p}$ and $\eta_{C F T}$. Similarly,

$$
W_{\mu}(\vec{x}, \vec{y})=W\left(\Delta\left(\frac{q}{p_{T}}\right), \eta_{C F T}\right) \times \delta\left(\Omega_{\mu}-\Omega_{p}\right)
$$

where $W\left(\Delta\left(\frac{q}{p_{T}}\right), \eta_{C F T}\right)$ is Gaussian function with parameters determined by fitting $1 / p_{T}$

$\Theta_{\text {parton }}(\vec{y})$ are the parton level cuts applied in order to avoid singularities in the matrix element calculation. All differential cross sections are calculated with these cuts, which are looser than those applied at the reconstructed level. They are summarized as $\Delta R\left(q_{i}, q_{j}\right)>0.5, p_{T}\left(q_{i}\right)>6 \mathrm{GeV},\left|\eta\left(q_{i}\right)\right|<3.5$ and no cuts are applied to lepton or neutrino.

$\int d \vec{y} d q_{1} d q_{2}$ is an integration over the phase space of final state partons with $\vec{y}$ and initial parton momenta $q_{1}$ and $q_{2}$. There are in total 14 independent spatial degrees of freedom for the lepton, neutrino, and two partons, giving

$$
d \vec{y}_{2}=d q_{1} d q_{2} d|p|_{\ell} d \Omega_{\ell} d|p|_{\nu} d \Omega_{\nu} d|p|_{q 1} d \Omega_{q 1} d|p|_{q 2} d \Omega_{q 2}
$$

However, 4 degrees of freedom are removed from the integration by the $\delta$ functions in the two jet transfer functions, which assumes the angles of matched jets and partons are well measured. Similarly, 2 more degrees of freedom are removed because of the well measured lepton angles. According to energy and momentum conservation, 4 more degrees of freedom are removed by eliminating $d|p|_{\nu} d \Omega_{\nu}$ and reducing $d q_{1} d q_{2}$ to $d p_{z}$. The phase spaces for different physics processes are then

$$
d \vec{y}_{\mathrm{W}+\mathrm{jets}}=d r_{W} d\left|p_{q 1}\right| d\left|p_{q 2}\right| d p_{z}^{\text {system }}
$$




$$
d \vec{y}_{\text {single top }}=d r_{\text {top }} d r_{W} d\left|p_{q 2}\right| d p_{z}^{\text {system }}
$$

$$
d \vec{y}_{\mathrm{WW}}=d r_{W} d r_{W} d\left|p_{q 2}\right| d p_{z}^{\text {system }}
$$

$$
d \vec{y}_{\mathrm{WZ}}=d r_{W} d r_{Z} d\left|p_{q 2}\right| d p_{z}^{s y s t e m}
$$

where $d r_{W}, d r_{\text {top }}$ and $d r_{H}$ are used to uniformly sample a Breit-Wigner distribution from the square of the invariant mass distribution for the $W$ boson, top quark and Higgs boson respectively. This minimizes the integration time because when the invariant masses $m_{l e p, \nu}, m_{l e p, \nu, b}$ and $m_{b, b}$ are far from the masses of $W, t$ and $H$ boson, the contributions of the matrix elements for these processes are negligible from the integration results. These multidimensional integrals are calculated using the VEGAS algorithm [112] of Monte Carlo integration techniques as implemented in the GNU Scientific Library [113].

The normalization constant $\sigma$ in Eq. 6.2 is defined as the integration of the differential cross section over the reconstructed level phase space, as

$$
\sigma=\sum_{i, j} \int d \vec{x} d \vec{y}\left[\frac{\sigma_{i, j}(\vec{y})}{\vec{y}} \times W(\vec{x}, \vec{y}) \times \Theta_{\text {cuts }}(\vec{x})\right]
$$

where $\Theta_{\text {cuts }}(\vec{x})$ represents the selection cuts at the reconstructed level, summarized as the following:

- Lepton $P_{T}>15 \mathrm{GeV}$

- Electron (muon) $|\eta|<1.1(2.0)$

- Missing $E_{T}>15 \mathrm{GeV}$ 
- Leading jet $P_{T}>25 \mathrm{GeV}$

- Leading jet $|\eta|<2.5$

- Second jet $P_{T}>20 \mathrm{GeV}$

- Second jet $|\eta|<3.5$

These cuts, slightly different from the selection cuts in the data, are included in the calculation to approximate the acceptance difference between signal and background events. The statistical uncertainty from the Monte Carlo integration is less than $1 \%$. Although these are 13 dimensional integrals, the integration time is trivial since they are performed once for all.

Note that practically there is no prior knowledge about assigning a parton to a jet. The event differential cross section has to be modified as

$$
\begin{aligned}
d \sigma(l, j 1, j 2) & =\alpha_{j 1 \rightarrow p 1} \alpha_{j 2 \rightarrow p 2} d \sigma(l, j 1 \rightarrow p 1, j 2 \rightarrow p 2) \\
& +\alpha_{j 2 \rightarrow p 1} \alpha_{j 1 \rightarrow p 2} d \sigma(l, j 2 \rightarrow p 1, j 1 \rightarrow p 2)
\end{aligned}
$$

where the $\alpha$ parameters in this analysis are determined using the b-tagging informa-

tion and tag rate functions. For example, given one jet tagged and the other one not in the two jets events of $W c g$ hypothesis, the differential cross section would be

$$
\begin{array}{r}
d \sigma_{W c g}(l, j 1, j 2)=\epsilon_{c}(j 1)\left(1-\epsilon_{l}(j 2)\right) d \sigma_{W c g}(l, j 1 \rightarrow c, j 2 \rightarrow g) \\
+\epsilon_{c}(j 2)\left(1-\epsilon_{l}(j 1)\right) d \sigma_{W c g}(l, j 1 \rightarrow g, j 2 \rightarrow c)
\end{array}
$$

where $\epsilon_{c}$ and $\epsilon_{l}$ are the tag rate functions.

\subsubsection{The Matrix Element Discriminant}

The discriminant for $W H$ signal event in this analysis is built as

$$
D_{W H}(\vec{x})=\frac{P_{W H}(\vec{x})}{P_{W H}(\vec{x})+P_{B}(\vec{x})}
$$


where the background probability density function $P_{B}(\vec{x})$ is defined as

$$
\begin{aligned}
P_{B}(\vec{x}) & =C_{W b b} P_{W b b}(\vec{x})+C_{W c g} P_{W c g}(\vec{x})+C_{W g g} P_{W g g}(\vec{x}) \\
& +C_{W W} P_{W W}(\vec{x})+C_{W Z} P_{W Z}(\vec{x})+C_{t b} P_{t b}(\vec{x})+C_{t q b} P_{t q b}(\vec{x})
\end{aligned}
$$

where $C_{W b b}, C_{W c g}, C_{W g g}, C_{W W}, C_{W Z}, C_{t b}$ and $C_{t q b}$ are the relative background fractions, which represent the contribution of each background process to the total background and satisfy $C_{W b b}+C_{W c g}+C_{W g g}+C_{W W}+C_{W Z}+C_{t b}+C_{t q b}=1$.

The corresponding matrix elements for $t \bar{t}$ and multijet events are not available in this analysis. An approximation is performed by assigning these events to the most similar background processes which have their dedicated matrix elements. The discriminant distributions of $t \bar{t}$ and multijet processes are compared to other backgrounds with the least $\chi^{2}$ test. The results are shown in Tab. 6.1.3.

\begin{tabular}{||l|c||}
\hline \hline The background process without matrix element & The most similar background process \\
\hline$t \bar{t} \rightarrow \ell+$ jets & $t b$ \\
$t \bar{t} \rightarrow \ell \ell$ & $t b$ \\
multijet & $W b b, W c g$ and $W g g$ \\
\hline \hline
\end{tabular}

Table 6.1: For three of the background processes in the analysis, $t \bar{t} \rightarrow \ell+$ jets, $t \bar{t} \rightarrow \ell \ell$ and multijet, there is no dedicated matrix element. Using a $\chi^{2}$ we determined the background sample with a matrix element which is most similar to each of the three. For the purpose of determining the constants in the background probability density function $t \bar{t} \rightarrow \ell+$ jets and $t \bar{t} \rightarrow \ell \ell$ are treated as $t b$ and multijet events are treated as $W+$ jets.

The seven relative fractions of the background events can be optimized to give the best sensitivity to the Higgs signal. However, it is time consuming to do the six dimensional optimization. The $C_{W W}, C_{W Z}, C_{t b}$ and $C_{t q b}$ are fixed according to the ratios of their expected number of events to those of the total background processes, in both single and double b-tagged samples. The rest of $C_{W b b}, C_{W c g}$ and $C_{W g g}$ are optimized using a 2-dimensional grid search to find the values which would give the best expected upper limit on $\sigma(W H) \times B R(H \rightarrow b \bar{b})$ at $95 \%$ C.L.. Two examples of optimization are shown in Fig. 6.3 and Fig. 6.4. The optimization is done 

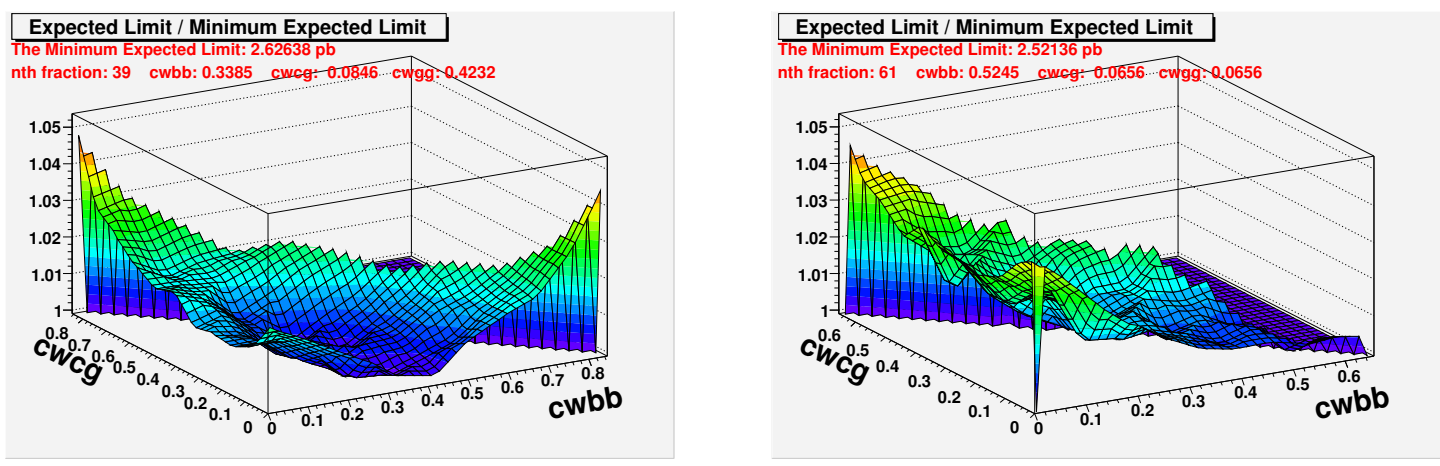

Figure 6.3: Result of the two-dimensional grid optimization for the constants $C_{W b b}$ and $C_{W c g}$ in the $e+$ jets channel for single and double tagged events. The result shown is for a Higgs mass of $115 \mathrm{GeV}$.
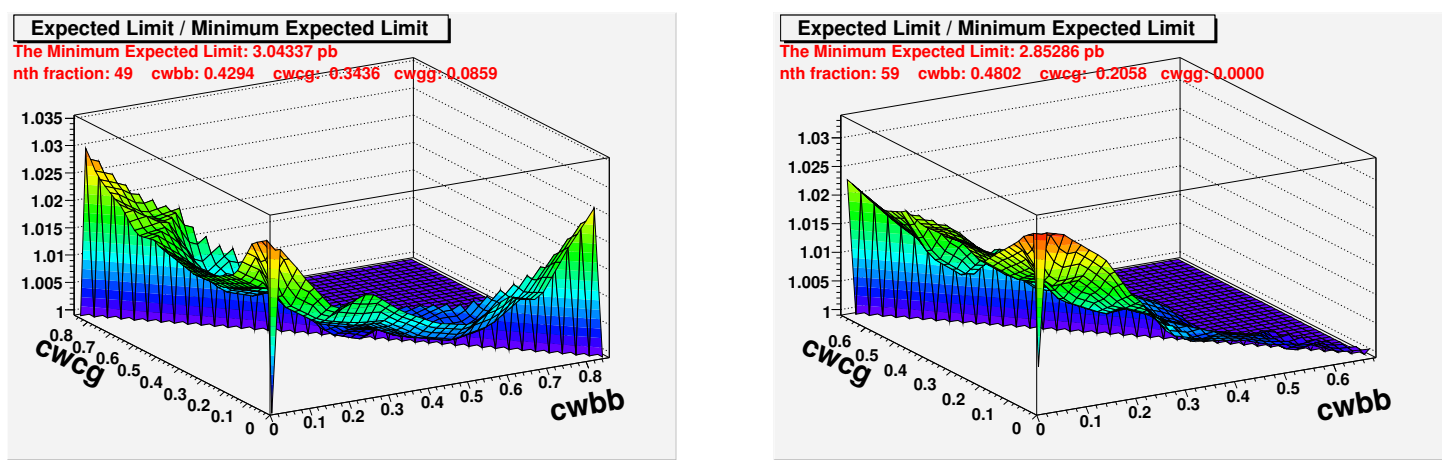

Figure 6.4: Result of the two-dimensional grid optimization for the constants $C_{W b b}$ and $C_{W c g}$ in the $\mu+$ jets channel for single and double tagged events. The result shown is for a Higgs mass of $115 \mathrm{GeV}$.

separately in the electron and muon final states with single b-tagged and double b-tagged jets, as well as averaged over different masses of the Higgs boson. The optimized background fractions are summarized in Tab. 6.1.3. The relative large values of $C_{t b}$ are due to the contributions of $t \bar{t}$ events. The discriminating power would be improved by adding the corresponding matrix elements to the background processes such as $t \bar{t}$ and multijet events.

Note that an approach of adding b-tagging information directly into the discriminant is tried and gives better performance without systematic uncertainties. However, the method turns out to be affected by the large shape systematic due to b-tagging information. It gives $\sim 8 \%$ worse performance and is thus abandoned. 


\begin{tabular}{||l||cccc||}
\hline \hline \multicolumn{4}{||c||}{ Optimized Background Fractions } \\
\hline & \multicolumn{2}{c||}{1 tag } & \multicolumn{2}{c||}{2 tags } \\
& Electron & Muon & Electron & Muon \\
\hline$C_{W b b}$ & 0.254 & 0.443 & 0.583 & 0.481 \\
$C_{W c g}$ & 0.197 & 0.243 & 0.022 & 0.124 \\
$C_{W g g}$ & 0.394 & 0.172 & 0.043 & 0.074 \\
$C_{W W}$ & 0.023 & 0.023 & 0.002 & 0.002 \\
$C_{W Z}$ & 0.010 & 0.010 & 0.027 & 0.025 \\
$C_{t b}$ & 0.092 & 0.079 & 0.314 & 0.286 \\
$C_{t q b}$ & 0.030 & 0.030 & 0.009 & 0.009 \\
\hline \hline
\end{tabular}

Table 6.2: Background fractions chosen for each analysis channel. The $C_{W b b}, C_{W c g}$ and $C_{W g g}$ constants are obtained from an optimization for the best expected limit on the $W H$ cross section. The optimization is done separately for each Higgs mass, and we use an average over the constants for the various Higgs masses in each of the four channels.

The $W+3 j e t$ event samples in the analysis are treated like $W+2 j e t$ events by integrating over only the leading two jets with $2 \rightarrow 4$ matrix elements. The distributions of ME discriminant for $W+2$ jet events with $m_{H}=115 \mathrm{GeV}$ are shown in Fig. 6.5. The data events are well described the simulated events. The $W H$ events are scaled up by a factor of 10 to be visible. They are clearly separated from the other background events and peak at the value close to 1 . The resulting ME discriminants are used as one of the input variables to the Neural Network as discussed in the next section.

\subsection{Artificial Neural Network}

\subsubsection{The Architecture of the Network}

The Artificial Neural Network (ANN) is further used in this analysis to correlate the kinematic information of the physics objects to separate the signal events from the background events. ANN consists of groups of interconnected artificial neurons; each neuron gives a certain response to input signals. The entire network maps a set of input variables $\vec{x}$ into a set of output variables $\vec{y}$, where the mapping is nonlinear if any of the neurons has nonlinear response to its input. The feed-forward multilayer 
perceptron of the TMultiLayerPerceptron class implemented in TMVA [114] of the ROOT framework [115] is selected for this analysis. The neuron response functions in the packages are provided with $\rho=\alpha \times \kappa$, where the synapsis function $\kappa$ could be any of linear sum, squared sum and absolute sum; the activation function $\alpha$ could be any of linear, sigmoid, tanh and radial functions.

The structure of the network is $8: 8: 1$, where 8 nodes in the first layer correspond to 8 input variables, the second layer is the hidden layer with 8 nodes, and one node of the final layer corresponds to the output. The desired output of the network is set to 1 for the signal and 0 for the background.

\subsubsection{The Input Variables}

The input variables have to be carefully selected for the neural network in order to achieve its maximal performance. In principle, the selected variables should describe the full kinematic information of the event and also be capable of discriminating the signal from the background. The discriminating power in this analysis is quantified by varying the values of input variables by $10 \% R M S$. The difference of the output represents the discriminating power of the input variable. An example of studies on the nine input variables is shown in Fig. 6.6. As can be seen in the plots, the $\eta($ leadingjet $), \eta($ secondleadingjet $)$ and $m_{T}\left(\right.$ lepton, $\left.\mathscr{H}_{T}\right)$ have little discrimination power and are thus discarded. Therefore, combined the dijet invariant mass, ME discriminant and the rest of the six variables, the list of final input variables to the neural network is:

- invariant mass of dijet $m_{T}($ jet 1, jet 2$)$

- Matrix Element discriminant

- $p_{T}($ leading jet $), p_{T}($ second leading jet $)$ 
- $\Delta R($ jet 1, jet 2$), \Delta \phi($ jet 1, jet 2$)$

- $p_{T}\left(\right.$ lepton $-\mathbb{E}_{T}$ system $)$

These input variables, except for the ME discriminant which has been shown the previous section, are shown separately for both electron and muon final states with $W+2$ jet events, as in Fig. 6.7, 6.8, 6.9 and 6.10. The single b-tagged events are shown in Fig. 6.11, 6.12, 6.13 and 6.14, while the double b-tagged events are shown in Fig. 6.15, 6.16, 6.17 and 6.18. The distributions of the data events are consistent with those of the simulated events.

\subsubsection{The Neural Network Training and the Output}

The training of the neural network is back propagated using the Broyden, Fletcher, Goldfarb, Shanno (BFGS) algorithm, which is built in TMVA. Given that the desired output of the neural network is $\hat{y}$ ( 1 for the signal, 0 for the background), the error on the agreement of the network response with the desired output is defined as

$$
E\left(\vec{x}_{1}, \ldots, \vec{x}_{N} \mid \vec{w}\right)=\sum_{i=1}^{N} \frac{1}{2}\left(y_{i}-\hat{y}_{i}\right)^{2}
$$

where $i$ is the $i^{\text {th }}$ event out of the total $N$ training events, $\vec{x}_{i}$ is the set input variables for event $i, y_{i}$ is the output of the event $i$ and $\vec{w}$ is the set of weights in the neural network to be adjusted. The training process is to find the set of weights that minimizes the error. The set of weights $\vec{x}$ is updated after each training epoch, which is defined as a loop over all the training events.

In this analysis, $W H$ and $W b b$ events are used as signal and background events for the training. The samples are required to have the same size by reducing the larger sample with an event weight. The training samples are respectively further divided into two equal subsets, one called training events and the other called test events. The test events are used to monitor the performance of the network. When 
the training error is decreasing but the test error is increasing, overtraining may occur. The number of epochs for convergent training is finally selected to be 200 , with which the test error no longer decreases obviously as the number of training epochs is increasing.

The neural networks are trained separately for Run IIa and Run IIb event samples, electron and muon final states, single b-tagged and double b-tagged events and over different Higgs boson masses. The training events are then removed from the event samples used in the analysis. The data events and simulated events are finally fed into the trained neural networks. The combined distributions of the neural network output are shown in Fig. 6.21 (linear vertical scale) and Fig. 6.22 (logarithmic vertical scale). The distributions of the data events are well described by those of the simulated events. The $W H$ signal events, scaled up by a factor of 10 to be visible, are peaked at high values close to 1.0. 

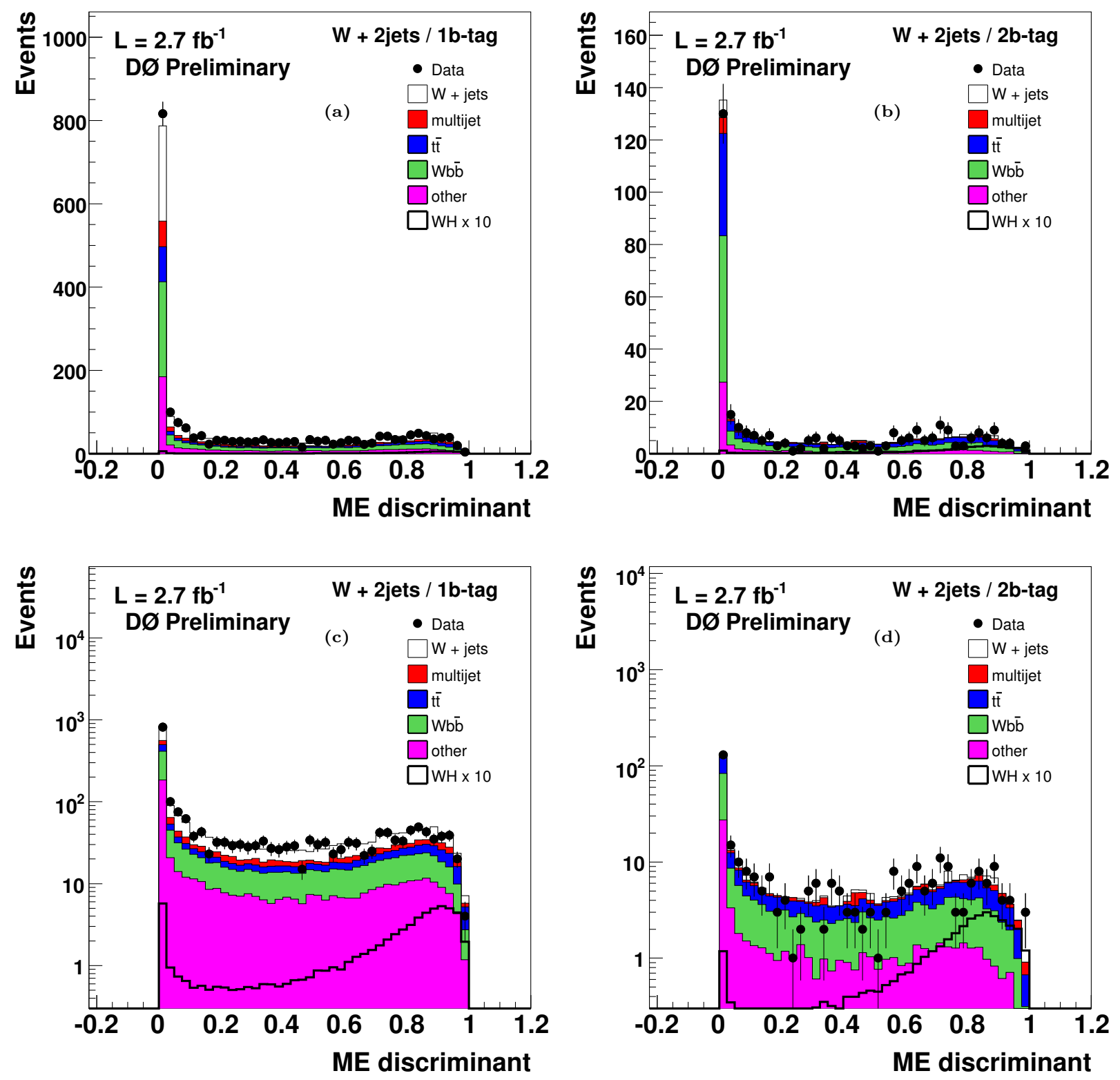

Figure 6.5: Distributions of the Matrix Element discriminant. The data events are compared to the simulated events for both single and double b-tagged jets. a) ME discriminant for the single b-tagged events. b) ME discriminant for the double b-tagged events. c) Log scale of figure (a). d) Log scale of figure (b). The simulation is normalized to the integrated luminosity of the data sample using the expected cross sections (absolute normalization) except for the $W+$ jets sample which is normalized on the "pre-tag sample" to the data, taking into account of all the other backgrounds. The $W H$ expected contribution which is scaled by a factor of 10 is peaking at high values of the ME discriminant as shown in c) and d). 


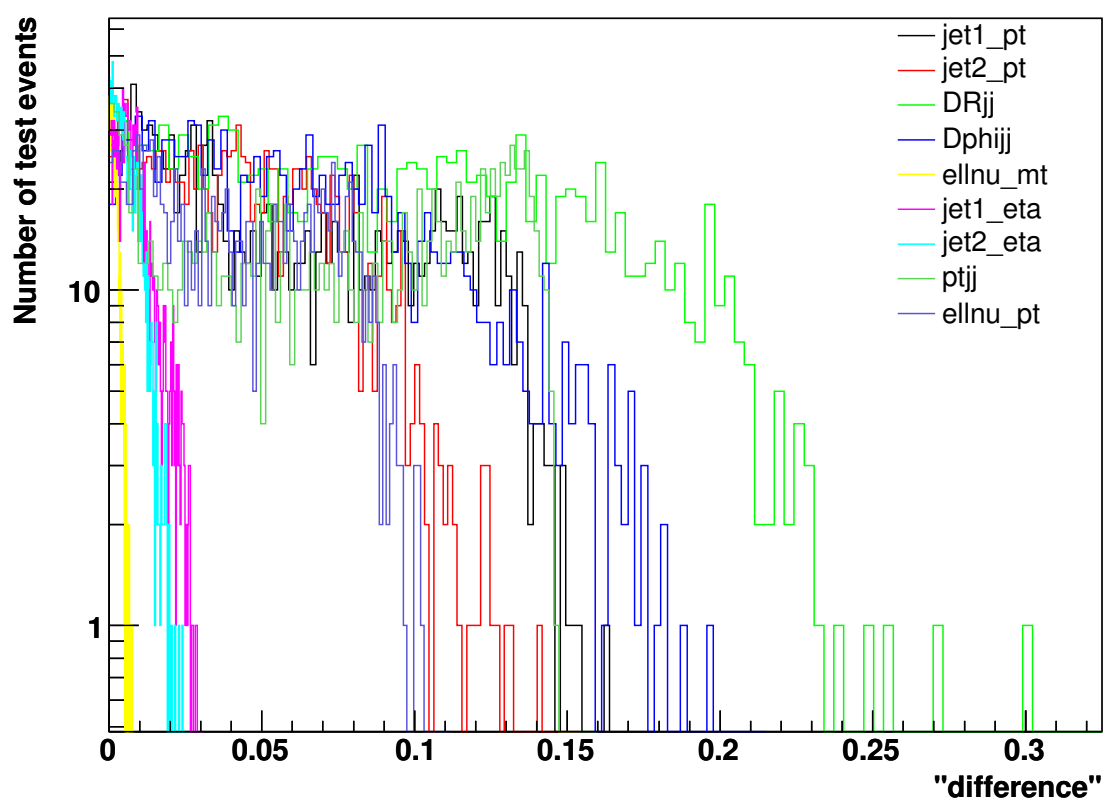

Figure 6.6: Discriminating power represented by the neural network output difference for each input variable in a nine-input neural network to separate $W H$ signal events from from $W b b$ background events. 

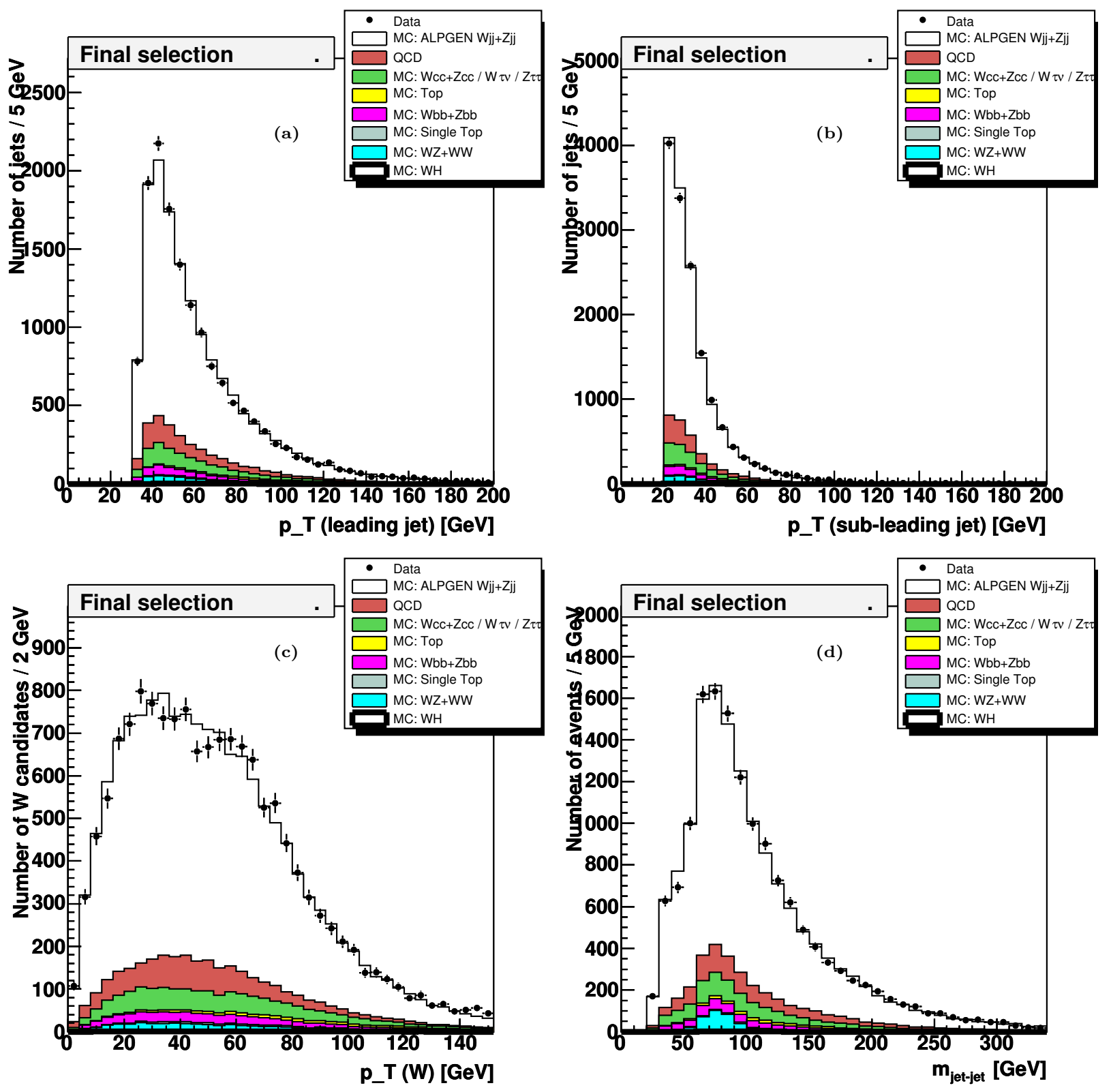

Figure 6.7: Distributions of the input variables for the neural network, of the $W+2$ jet electron $(\mathrm{CC}+\mathrm{EC})$ analysis with the Run IIb data set before $b$-tagging: a) $p_{T}$ of leading jet, $\mathrm{b}$ ) $p_{T}$ of sub-leading jet, c) $p_{T}$ of $e-\mathbb{E}_{T}$ system, d) dijet invariant mass. 

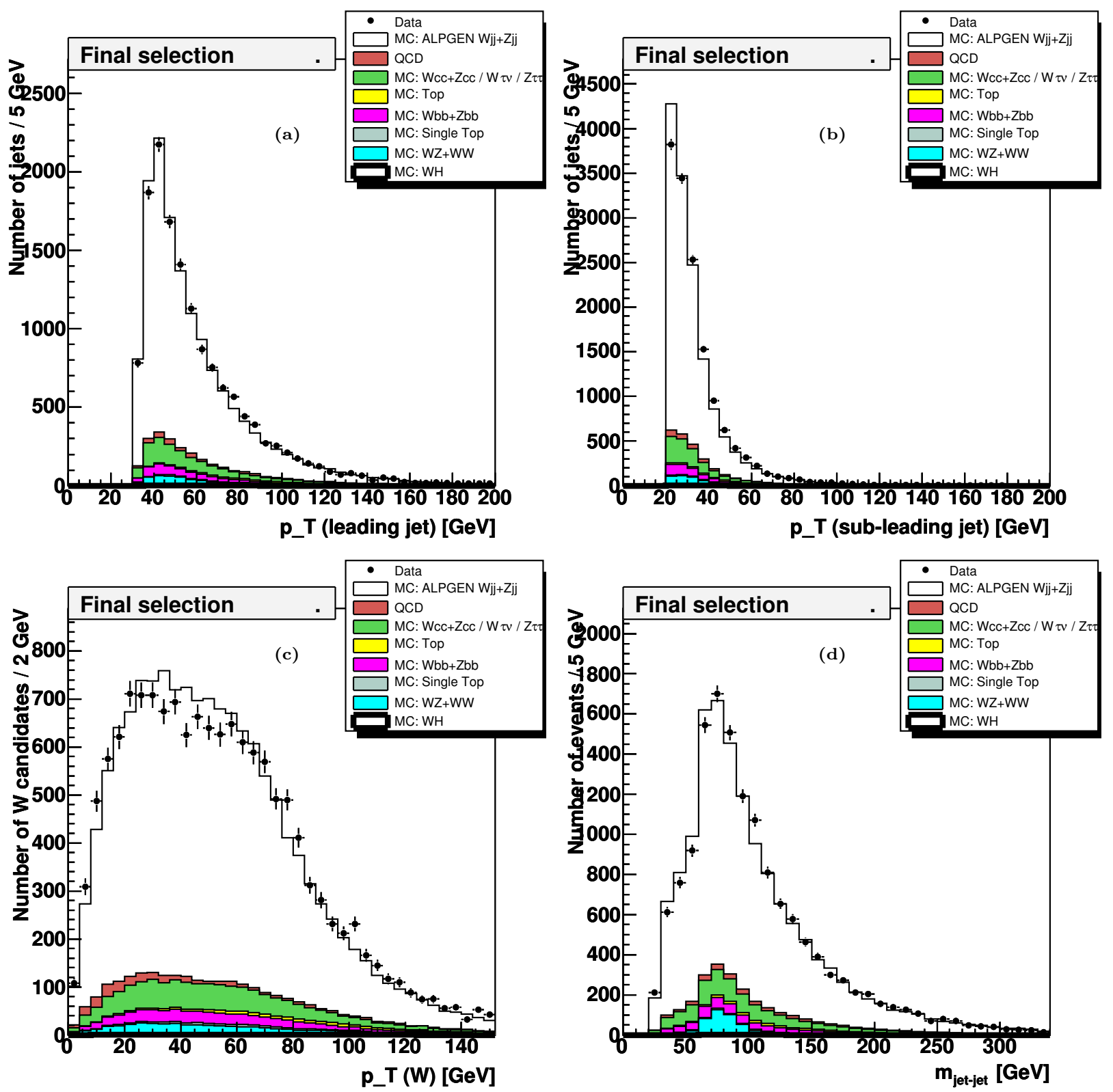

Figure 6.8: Distributions of the input variables for the neural network, of the $W+2$ jet muon analysis with the Run IIb data set before $b$-tagging: a) $p_{T}$ of leading jet, b) $p_{T}$ of sub-leading jet, c) $p_{T}$ of $\mu$ - $\mathbb{E}_{T}$ system, d) dijet invariant mass. 

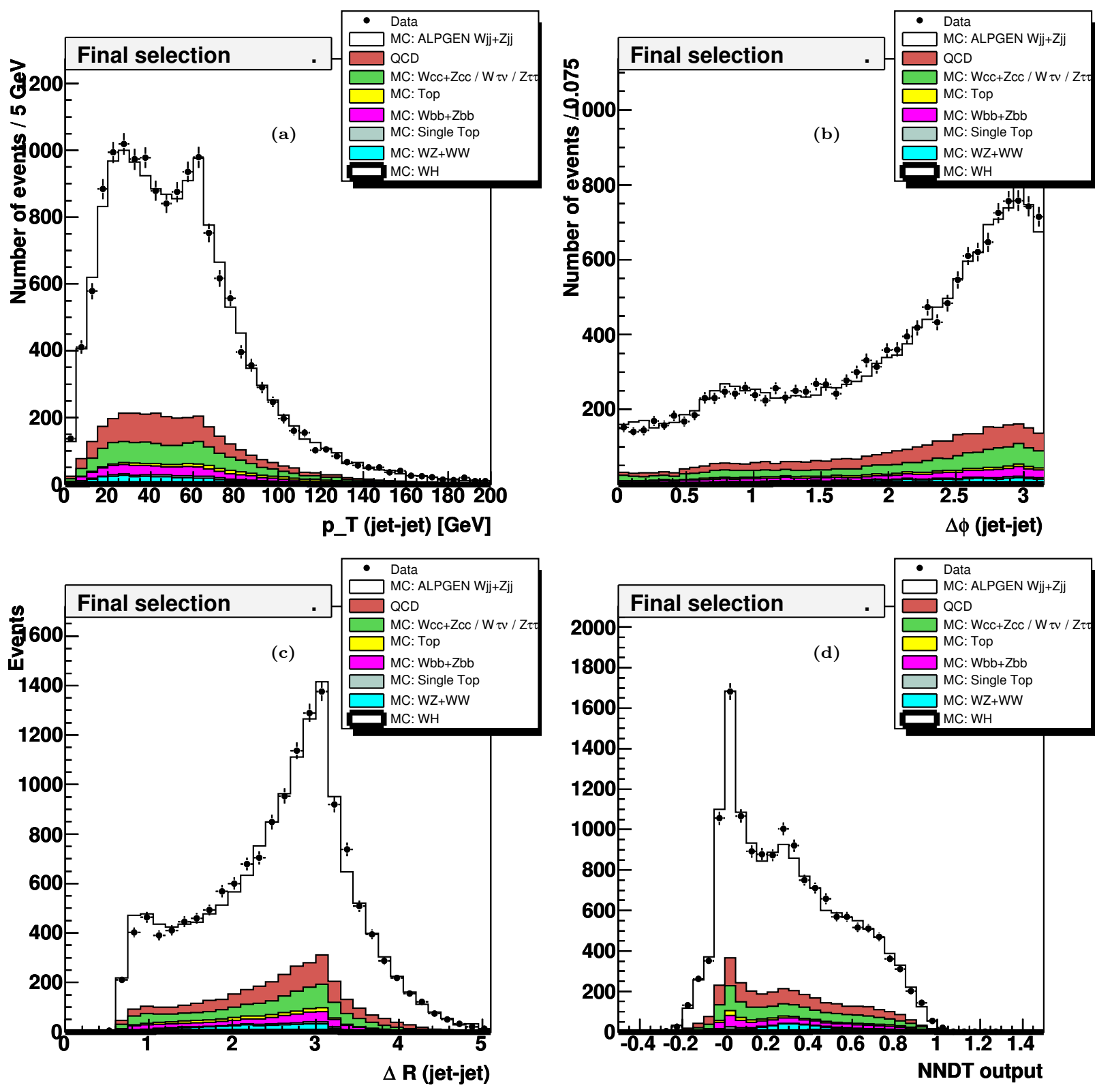

Figure 6.9: Distributions of the input variables for the neural network and the neural network output of the $W+2$ jet electron $(\mathrm{CC}+\mathrm{EC})$ analysis with the Run IIb data set, before $b$-tagging: a) $p_{T}$ of dijet system, b) $\Delta \phi$ between two leading jets, c) $\Delta R$ between two leading jets, d) neural network output (using the $m_{H}=115 \mathrm{GeV}$, double $b$-tag neural network). 

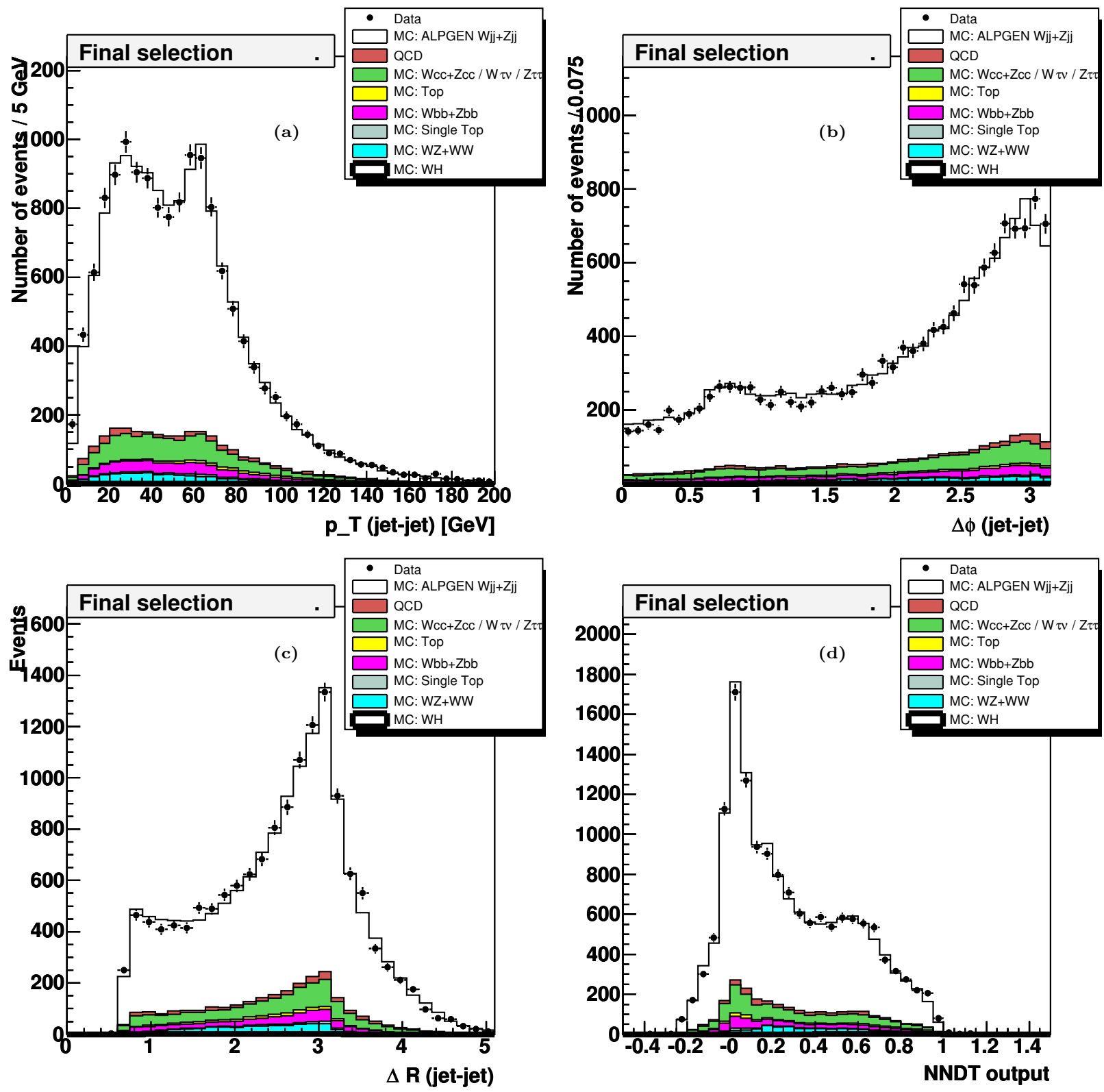

Figure 6.10: Distributions of the input variables for the neural network and the neural network output of the $W+2$ jet muon analysis with the Run IIb data set, before $b$-tagging: a) $p_{T}$ of dijet system, b) $\Delta \phi$ between two leading jets, c) $\Delta R$ between two leading jets, d) neural network output (using the $m_{H}=115 \mathrm{GeV}$, double $b$-tag neural network). 

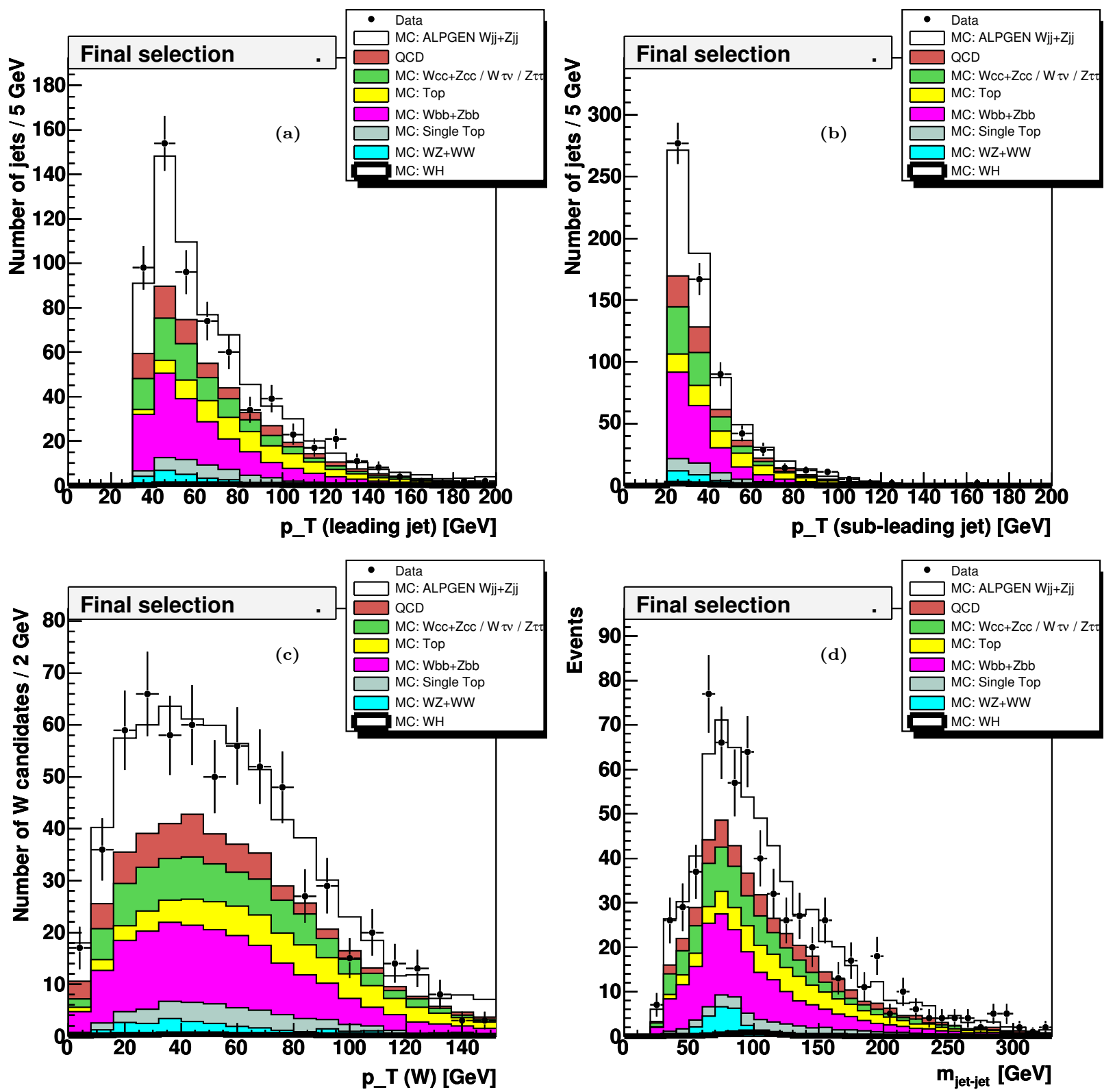

Figure 6.11: Distributions of the input variables for the neural network of the $W+2$ jet electron $(\mathrm{CC}+\mathrm{EC})$ analysis with the Run IIb data set, for events with a single $b$-tag (exclusive): a) $p_{T}$ of leading jet, b) $p_{T}$ of sub-leading jet, c) $p_{T}$ of $e-E_{T}$ system, d) dijet invariant mass. 

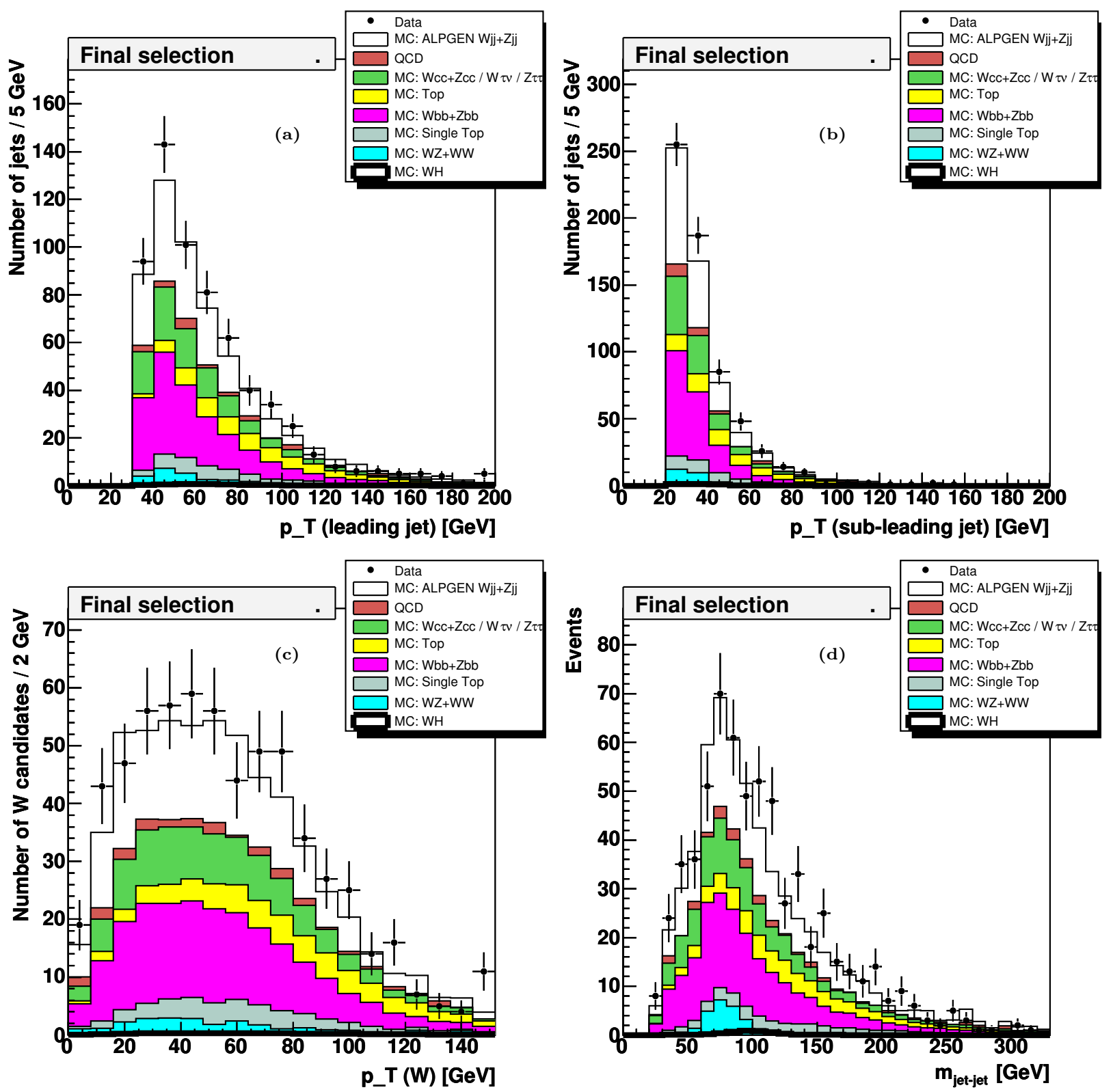

Figure 6.12: Distributions of the input variables for the neural network of the $W+2$ jet muon analysis with the Run IIb data set, for events with a single $b$-tag (exclusive): a) $p_{T}$ of leading jet, b) $p_{T}$ of sub-leading jet, c) $p_{T}$ of $\mu-E_{T}$ system, d) dijet invariant mass. 

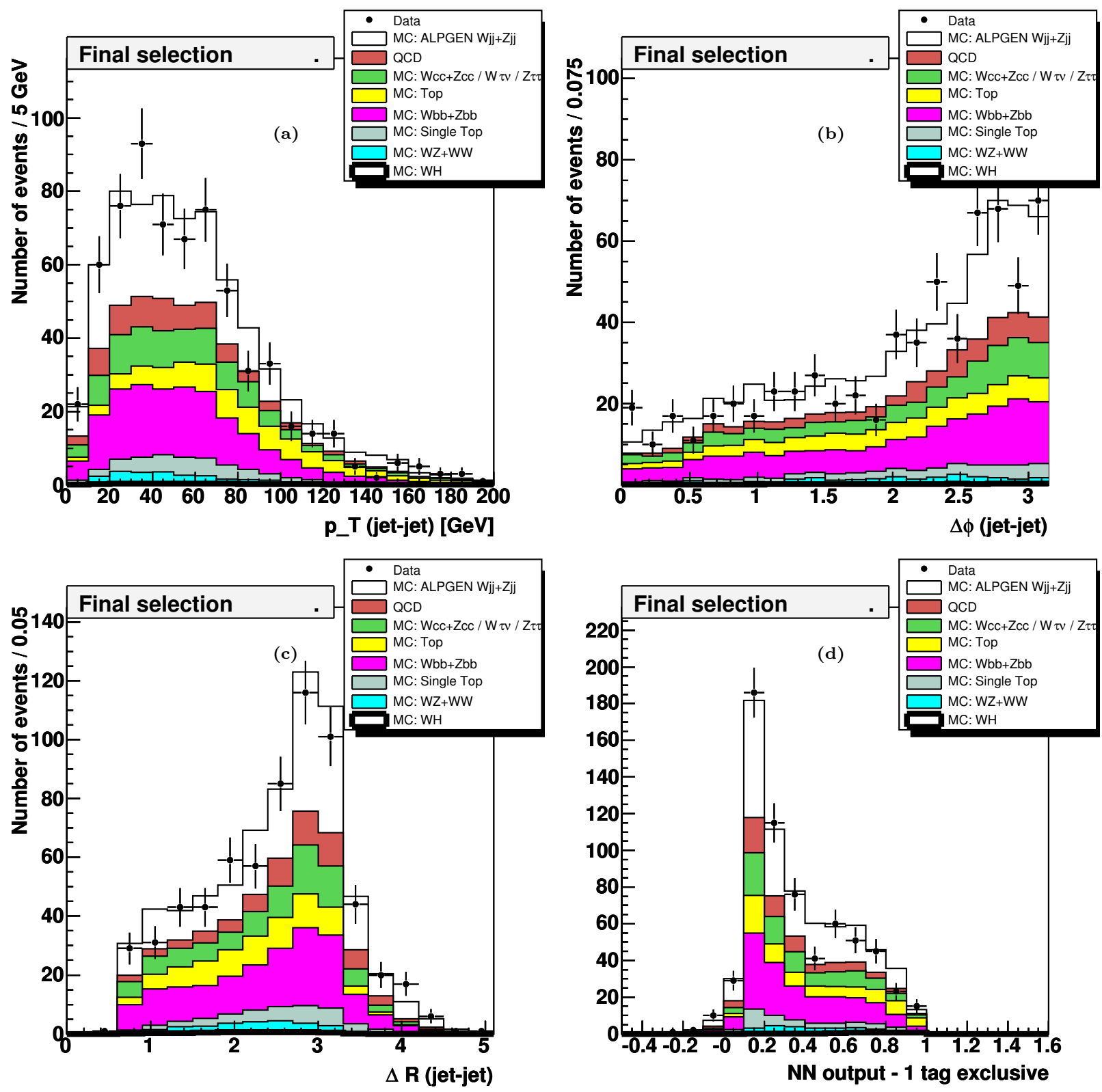

Figure 6.13: Distributions of the input variables for the neural network and the neural network output of the $W+2$ jet electron $(\mathrm{CC}+\mathrm{EC})$ analysis with the Run IIb data set, for events with a single $b$-tag (exclusive): $b$-tagging: a) $p_{T}$ of dijet system, b) $\Delta \phi$ between two leading jets, c) $\Delta R$ between two leading jets, d) neural network output (using the $m_{H}=115 \mathrm{GeV}$, single $b$-tag neural network). 

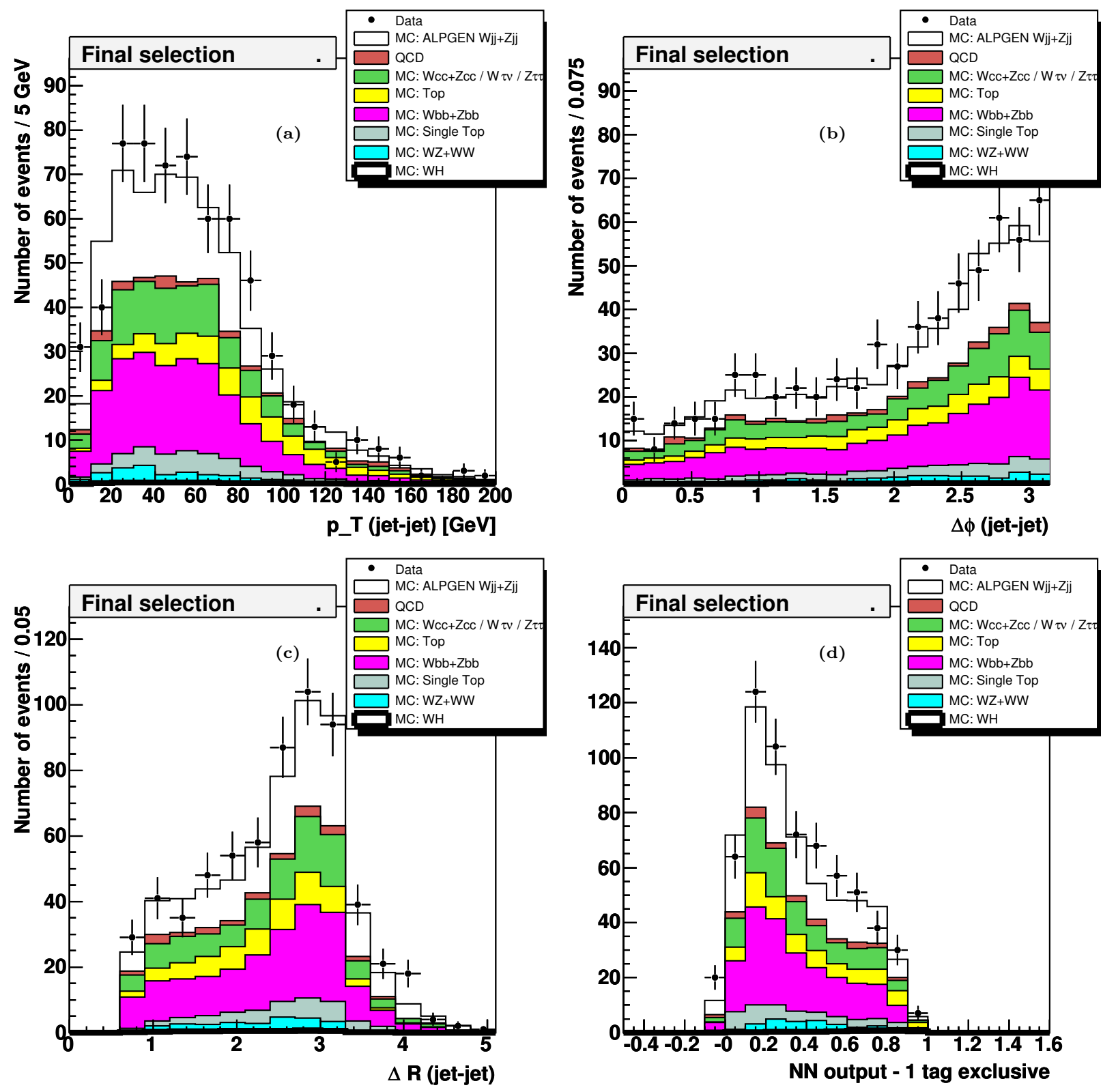

Figure 6.14: Distributions of the input variables for the neural network and the neural network output of the $W+2$ jet muon analysis with the Run IIb data set, for events with a single $b$-tag (exclusive): $b$-tagging: a) $p_{T}$ of dijet system, b) $\Delta \phi$ between two leading jets, c) $\Delta R$ between two leading jets, d) neural network output (using the $m_{H}=115$ $\mathrm{GeV}$, single $b$-tag neural network). 

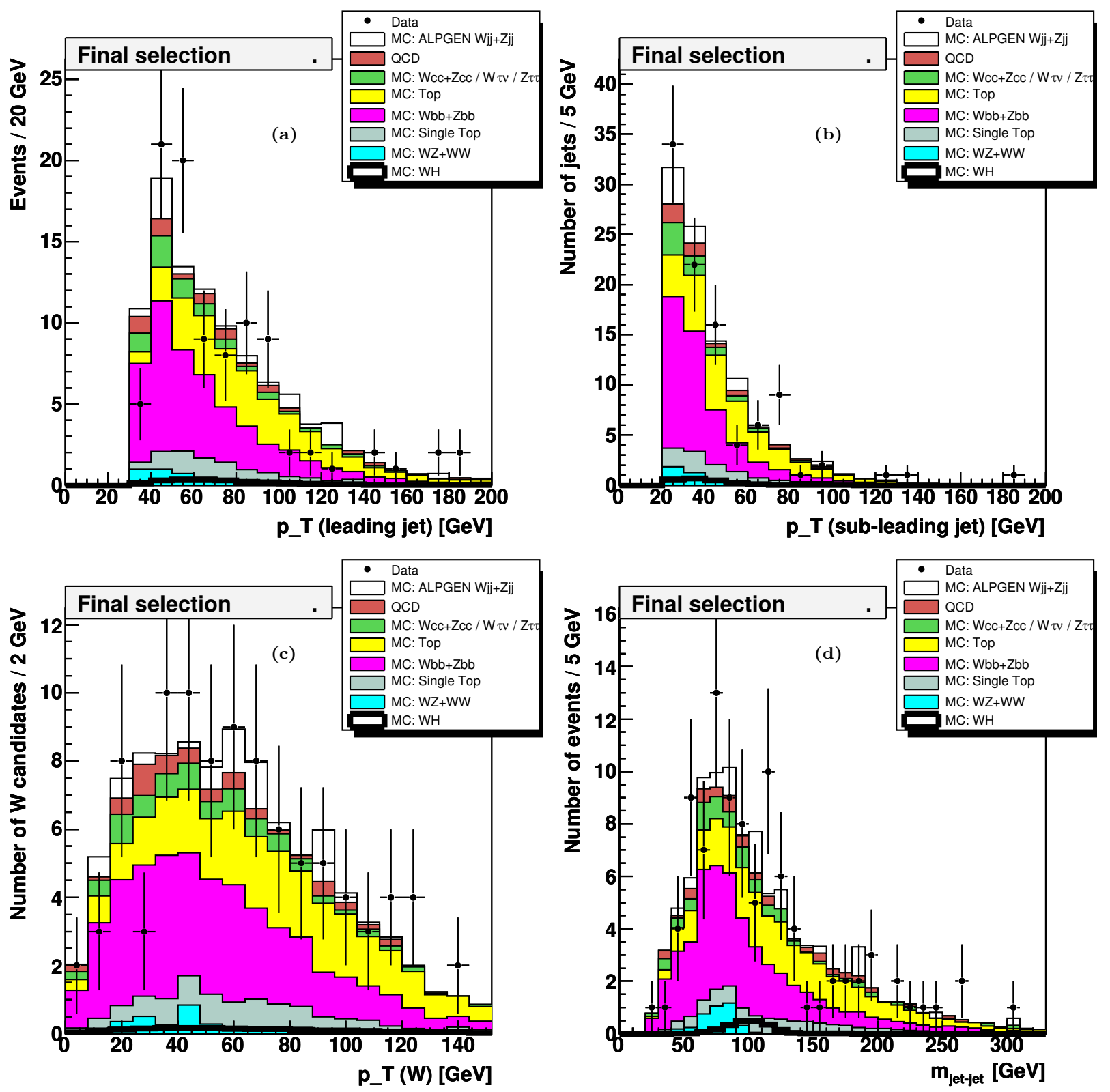

Figure 6.15: Distributions of the input variables for the neural network of the $W+2$ jet electron $(\mathrm{CC}+\mathrm{EC})$ analysis with the Run IIb data set, for events with a double $b$-tag: a) $p_{T}$ of leading jet, b) $p_{T}$ of sub-leading jet, c) $p_{T}$ of $e-\mathbb{E}_{T}$ system, d) dijet invariant mass. 

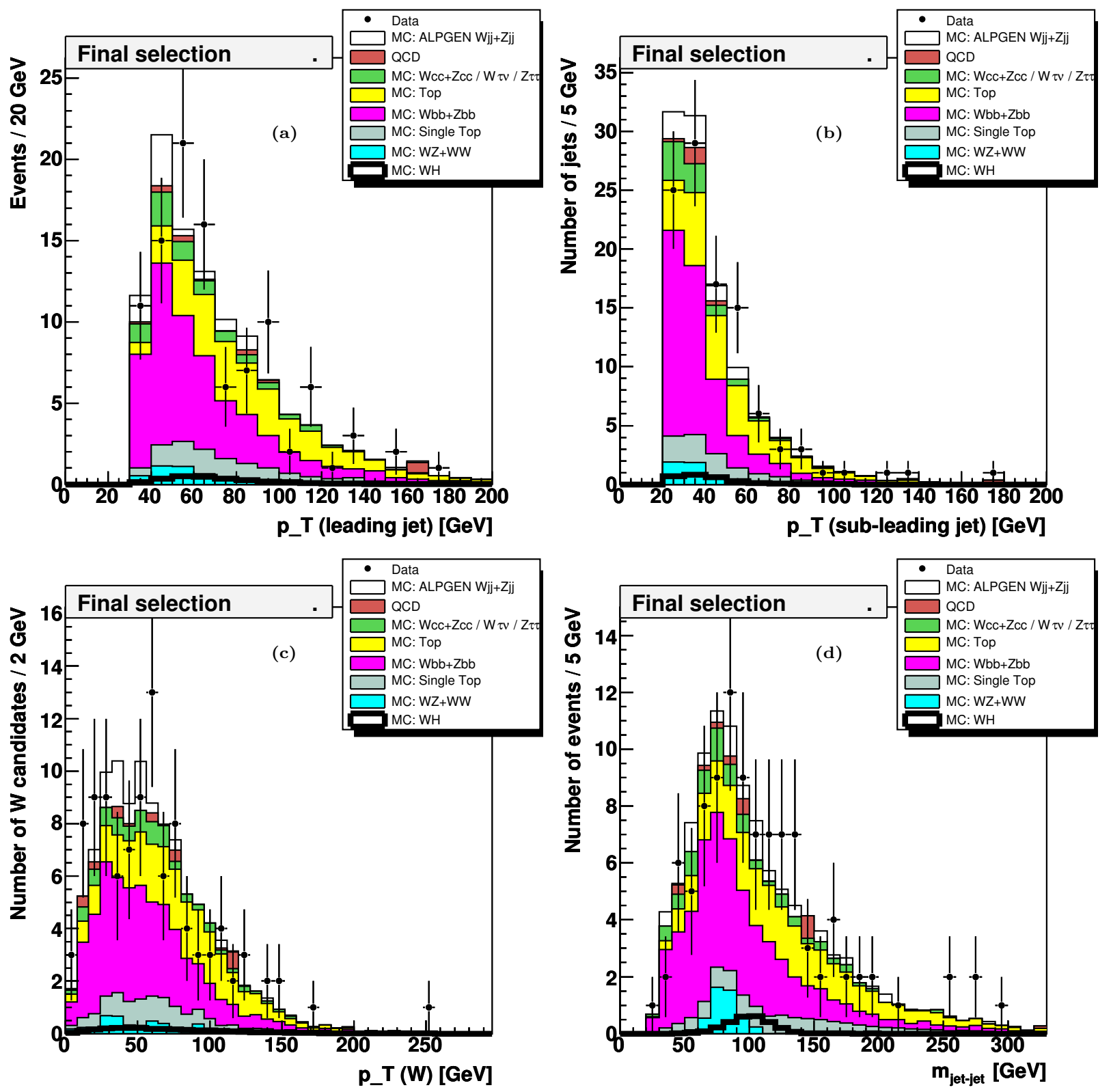

Figure 6.16: Distributions of the input variables for the neural network of the $W+2$ jet muon analysis with the Run IIb data set, for events with a double $b$-tag: a) $p_{T}$ of leading jet, b) $p_{T}$ of sub-leading jet, c) $p_{T}$ of $\mu$ - $\mathbb{E}_{T}$ system, d) dijet invariant mass. 

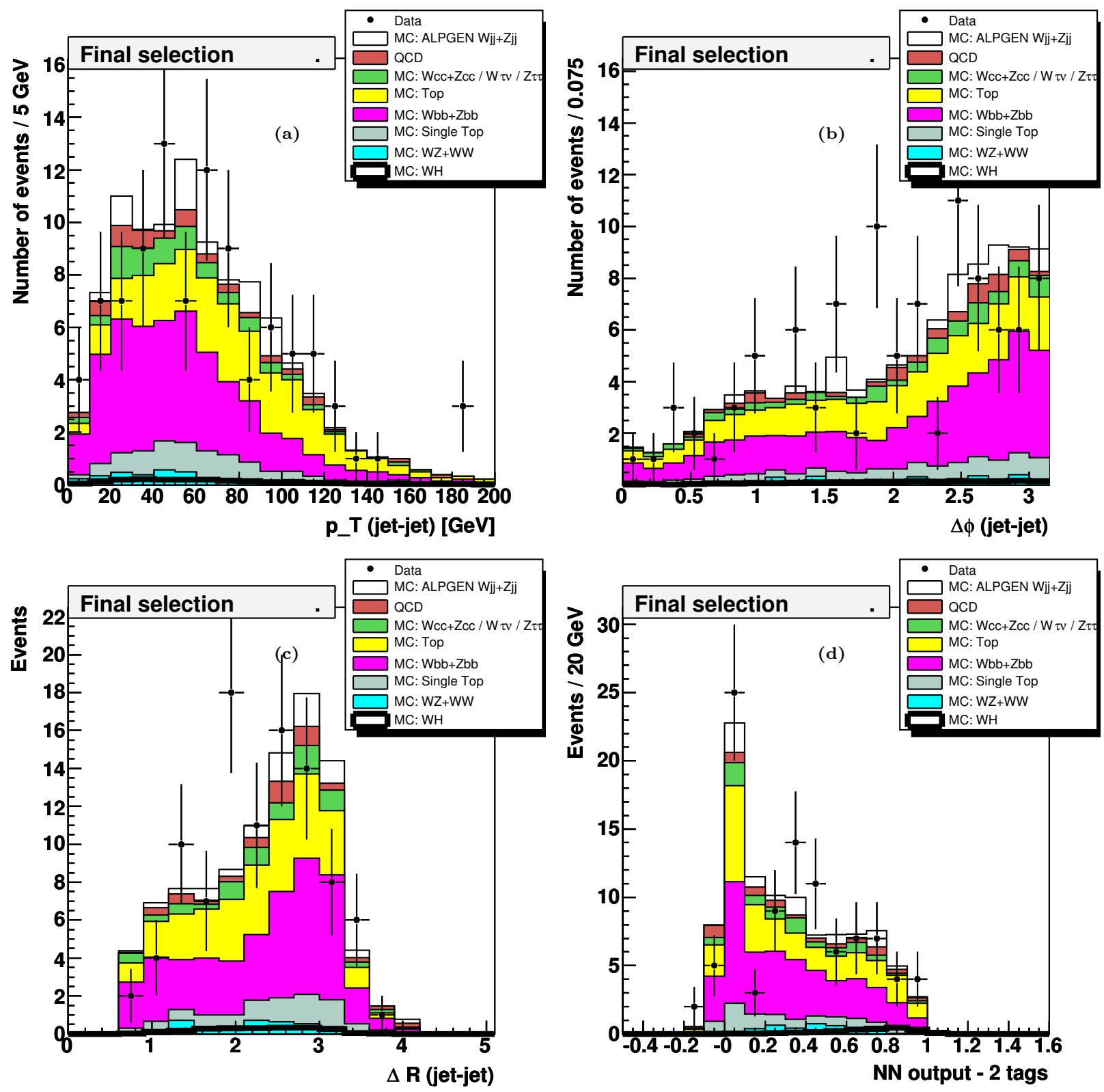

Figure 6.17: Distributions of the input variables for the neural network and the neural network output of the $W+2$ jet electron $(\mathrm{CC}+\mathrm{EC})$ analysis with the Run IIb data set, for events with a double $b$-tag: $b$-tagging: a) $p_{T}$ of dijet system, b) $\Delta \phi$ between two leading jets, c) $\Delta R$ between two leading jets, d) neural network output (using the $m_{H}=115 \mathrm{GeV}$, double $b$-tag neural network). 

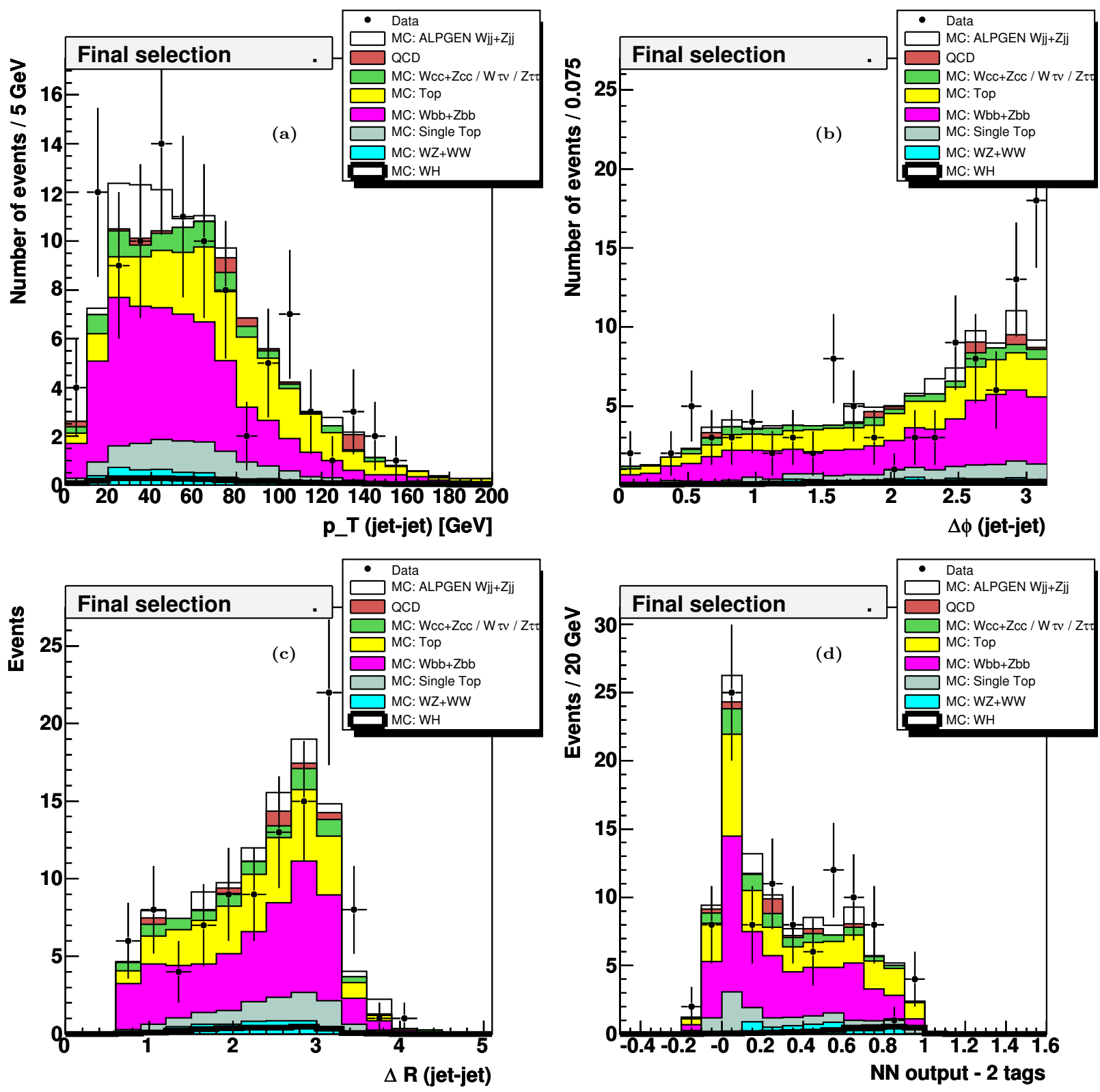

Figure 6.18: Distributions of the input variables for the neural network and the neural network output of the $W+2$ jet muon analysis with the Run IIb data set, for events with a double $b$-tag: $b$-tagging: a) $p_{T}$ of dijet system, b) $\Delta \phi$ between two leading jets, c) $\Delta R$ between two leading jets, d) neural network output (using the $m_{H}=115 \mathrm{GeV}$, double $b$-tag neural network). 

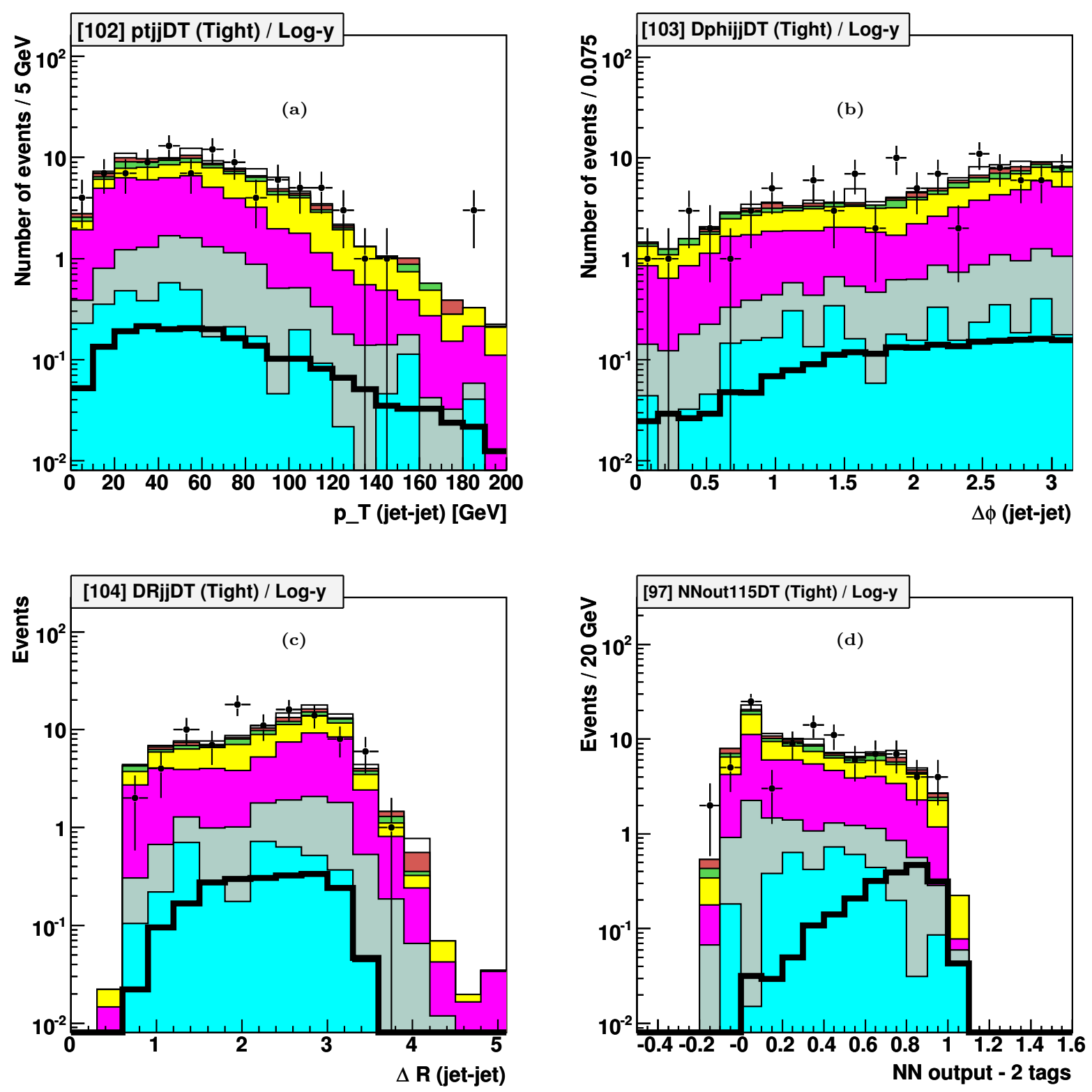

Figure 6.19: Distributions (Log-y) of the input variables for the neural network and the neural network output, of the $W+2$ jet electron analysis with the Run IIb data set, for events with a double $b$-tag: $b$-tagging: a) $p_{T}$ of dijet system, b) $\Delta \phi$ between two leading jets, c) $\Delta R$ between two leading jets, d) neural network output (using the $m_{H}=115 \mathrm{GeV}$, double $b$-tag neural network). 

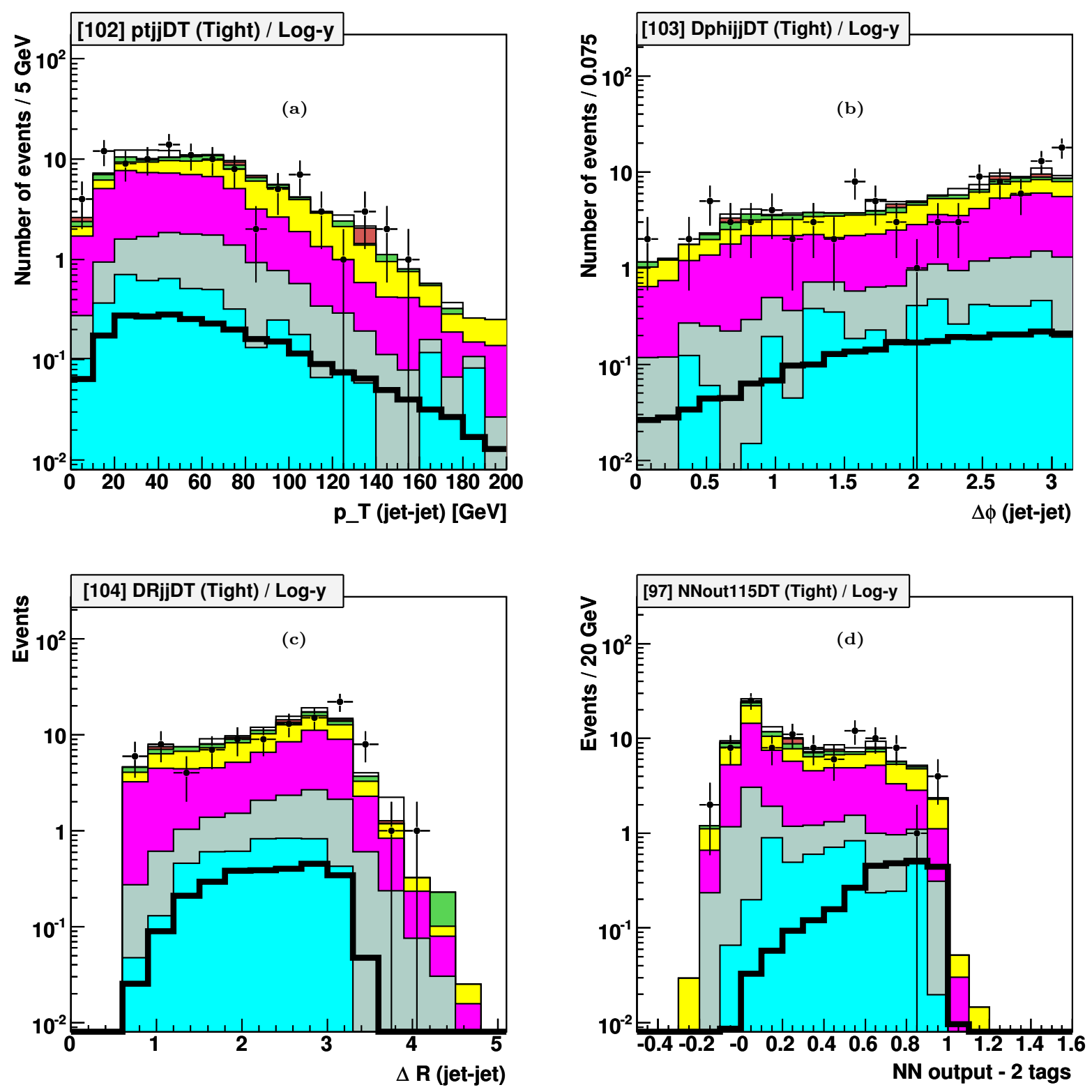

Figure 6.20: Distributions (Log-y) of the input variables for the neural network and the neural network output, of the $W+2$ jet muon analysis with the Run IIb data set, for events with a double $b$-tag: $b$-tagging: a) $p_{T}$ of dijet system, b) $\Delta \phi$ between two leading jets, c) $\Delta R$ between two leading jets, d) neural network output (using the $m_{H}=115 \mathrm{GeV}$, double $b$-tag neural network). 

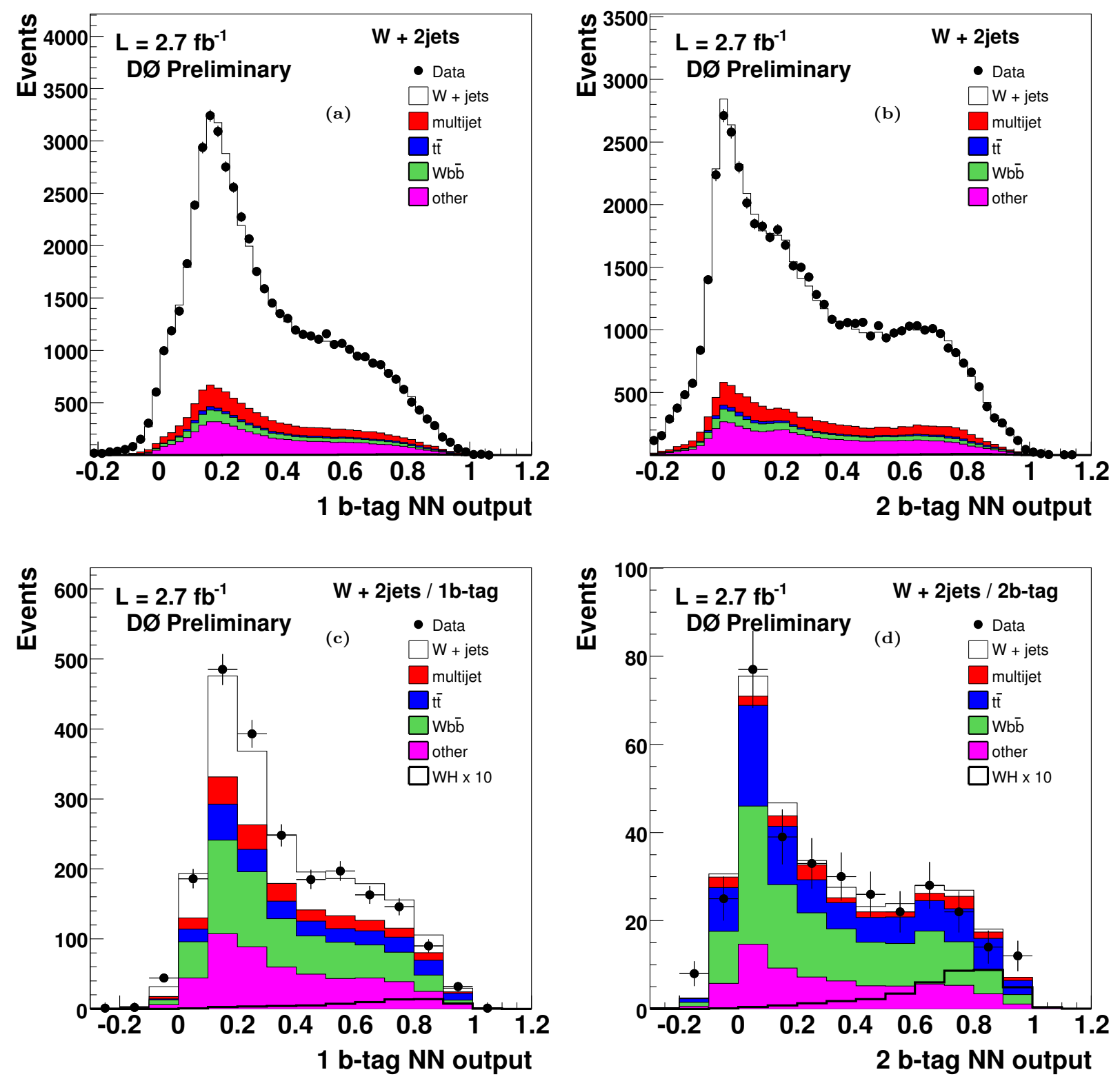

Figure 6.21: Distributions (with linear vertical scale) of the neural network output compared with the simulated expectation. a) before $b$-tagging for the single-tag NN. b) before $b$ tagging for the double-tag NN. c) in the single $b$-tagged sample for the single-tag NN. d) in the double $b$-tagged sample for the double-tag NN ; The simulation is normalized to the integrated luminosity of the data sample using the expected cross sections (absolute normalization) except for the $W+$ jets sample which is normalized on the "pre-tag sample" to the data, taking into account all the other backgrounds. The $W H$ expected contribution for $m_{H}=115 \mathrm{GeV}$, multiplied by a factor 10 , is peaking at high values of the NN output as shown in c) and d). 

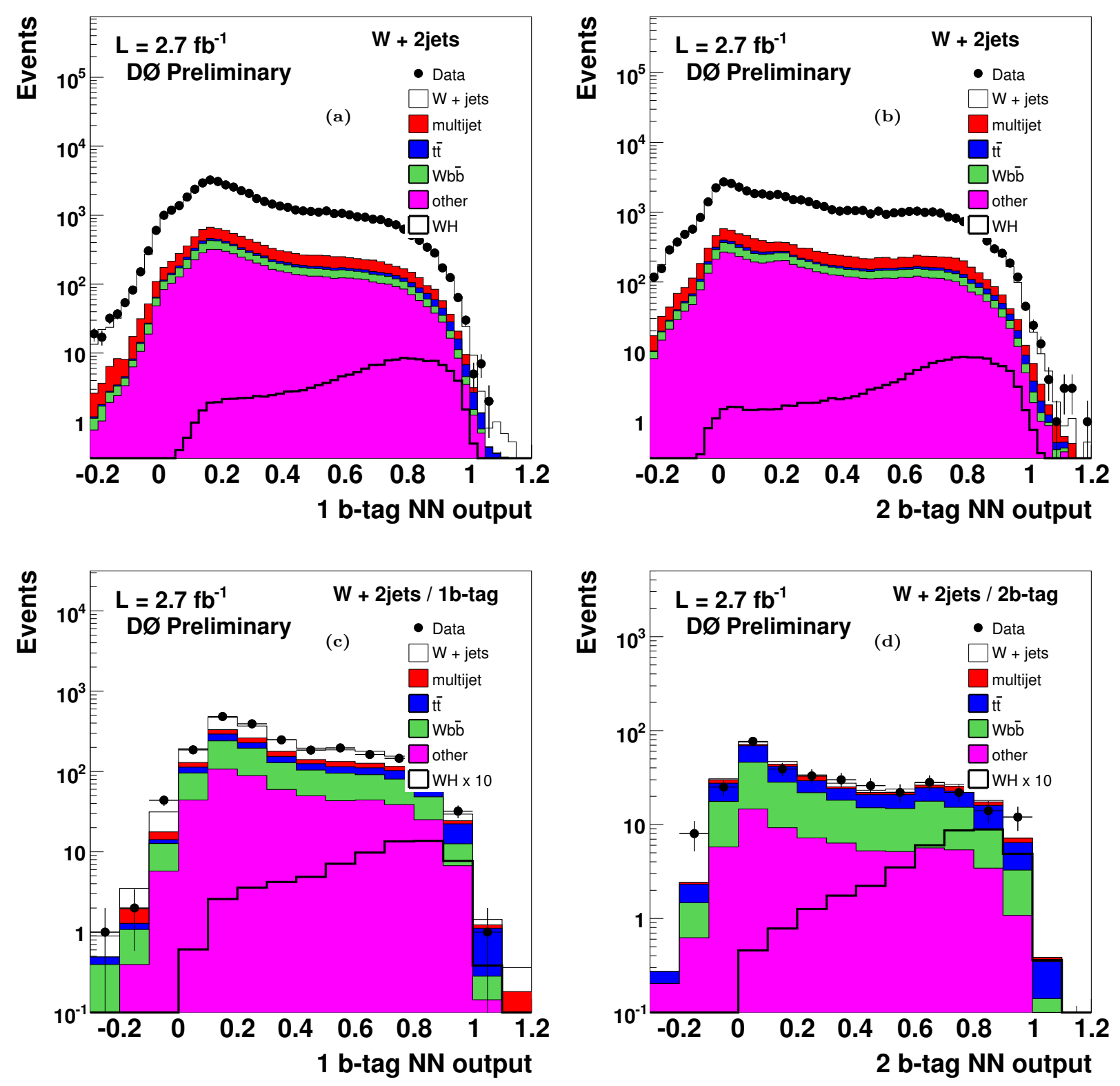

Figure 6.22: Distributions (with logarithmic vertical scale) of the neural network output compared with the simulated expectation. a) before $b$-tagging for the single-tag NN. b) before $b$-tagging for the double-tag NN. c) in the single $b$-tagged sample for the single-tag NN. d) in the double $b$-tagged sample for the double-tag NN ; The simulation is normalized to the integrated luminosity of the data sample using the expected cross sections (absolute normalization) except for the $W+$ jets sample which is normalized on the "pre-tag sample" to the data, taking into account all the other backgrounds. The $W H$ expected contribution for $m_{H}=115 \mathrm{GeV}$, multiplied by a factor 10 , is peaking at high values of the NN output as shown in c) and d). 


\section{CHAPTER VII}

\section{Results of WH Production Search}

There is no evidence of the Higgs boson observed in the data. Thus we use the output of the neural network to set upper limits on the cross section of $p \bar{p} \rightarrow$ $W H \rightarrow l \nu b \bar{b}$. The systematic uncertainties as well as their correlations are taken into account when the limits on the production cross sections are evaluated using a modified frequentist approach. Both the experimental and theoretical uncertainties are discussed in Section 7.1. The method of setting upper bounds on the cross sections is described in Section 7.2. A brief summary on this analysis and the results are made in the last Section 7.3.

\subsection{Systematic Uncertainties}

The experimental systematic uncertainties are obtained by shifting the central values of the variables with $\pm \sigma$, where $\sigma$ is the standard deviation representing the size of the uncertainty. All shifted variables are propagated through the full analysis to the distributions of the final variable. If the ratio of the shifted final variable to the original one is flat over the distributions, a constant number of systematic uncertainty is used in the limit calculation. Otherwise, if the ratio is not constant

over the distributions, it is fitted with a polynomial function, which is then used as a shaped systematic uncertainty in the limit calculation. 
The correlations of the systematic uncertainties among different event samples are summarized in Tab. 7.1, with the naming convention explained in Tab. 7.2. Most of the uncertainties in the 16 different event samples are correlated except for those specific to lepton type (electron or muon) and are derived for $W+2 j e t$ or $W+3 j e t$ events. The correlations are maintained through the limit calculation.

Various systematic uncertainties for the different physics processes with single and double b-tagged events are listed in Tables 7.3, 7.4, 7.5 and 7.6. They are summarized as:

- Uncertainties on the efficiencies of the triggers, as described in Section 5.1.1, are determined using the data samples. For the triggers used for the EM skims, the value is taken from the statistical error on the measurement of these trigger efficiencies, and determined to be $\sim 4 \%$. For the triggers used for MU skims, the uncertainty is in addition estimated by comparing the events fired by single muon triggers to the events fired by all the triggers, and finally added up to $\sim 5 \%$. The dependence on the shapes is also confirmed to be flat. The uncertainties on the efficiencies of lepton identification are determined in a similar way and estimated to be $\sim 5 \%$.

- Uncertainties of jet identification, JES and JetSSR add up to 6\%. The reweighting on the jet angular distributions due to $W+$ jets modeling gives another $5 \%$ to $W+$ jets events. An additional uncertainty of $7 \%$ is added to the $W+$ jets background accounting for the difference in the dijet mass distribution between data events and simulated events. The uncertainties on both the jet reweighting and dijet mass are shape dependent.

- Uncertainty of jet taggability is $3 \%$. For the b-tagging efficiencies, it is $4 \%$ on 
average for each heavy flavor jet, while it could be up to $25 \%$ for the light jets due to rescaling procedure of the scale factor to $W+j j$ event samples. This uncertainty is translated into $7 \%$ uncertainty on the total background of the single b-tagged events and is negligible for the double b-tagged events.

- Uncertainty on the cross sections of $W H$ and diboson production is $6 \%$, while it is $\sim 11 \%$ for top quark production. Due to the normalization of $W+j e t s$ as well as calculating HF factors, an uncertainty of $20 \%$ is assgined to $W+h$.f.jets events.

- Uncertainty on the multijet events is estimated by a variation of $\pm 15 \%$ (the uncertainty of the multijet fake rates) in the events sample where the lepton is back-to-back relative to a jet in the transverse plane. The uncertainty is determined to be $\sim 26 \%$.

- The overall experimental systematic uncertainty for $W H$ is approximately $14 \%$ for single and double b-tagged events. The uncertaintly on luminosity reweighting is $\sim 5 \%$, while the total uncertainty on luminosity measurement of $6.1 \%$ is treated separatedly in the analysis.

\subsection{Limits on WH Production Cross Sections}

The number of events (Tab. 5.16) in the data samples, as well as the distributions

of the dijet mass (Fig. 5.10 and 5.9) and neural network output (Fig. 6.21 and 6.22), are well described by the simulated background events. There is no excess of the 


\begin{tabular}{|c|c|c|c|c|c|c|c|c|}
\hline & $\begin{array}{l}\text { electron } \\
2 \text { jet-1b } \\
\text { IIa IIb }\end{array}$ & $\begin{array}{c}\text { electron } \\
2 \text { jet-2b } \\
\text { IIa IIb }\end{array}$ & $\begin{array}{c}\text { electron } \\
3 \text { jet-1b } \\
\text { IIa IIb }\end{array}$ & $\begin{array}{l}\text { electron } \\
\text { 3jet-2b } \\
\text { IIa IIb }\end{array}$ & $\begin{array}{c}\text { muon } \\
\text { 2jet-1b } \\
\text { IIa IIb }\end{array}$ & $\begin{array}{c}\text { muon } \\
\text { 2jet-2b } \\
\text { IIa IIb }\end{array}$ & $\begin{array}{c}\text { muon } \\
\text { 3jet-1b } \\
\text { IIa IIb }\end{array}$ & $\begin{array}{c}\text { muon } \\
\text { 3jet-2b } \\
\text { IIa IIb }\end{array}$ \\
\hline$\overline{\text { DZero_Lumi }}$ & $\overline{\mathrm{xx}}$ & $7 \mathrm{xx}$ & $\overline{\mathrm{x} \mathrm{x}}$ & $\begin{array}{l}\mathrm{x} x \\
\end{array}$ & $\overline{\mathrm{x} \mathrm{x}}$ & $\overline{\mathrm{xx}}$ & $\overline{\mathrm{x} \mathrm{x}}$ & $\overline{\mathrm{xx}}$ \\
\hline Lumi & $\mathrm{x} x$ & $\mathrm{x} x$ & $\mathrm{x} x$ & $\mathrm{x} x$ & $\mathrm{x} x$ & $\mathrm{x} x$ & $\mathrm{x} x$ & $\mathrm{x} x$ \\
\hline JESID & $\mathrm{x} x$ & $\mathrm{x} x$ & $\mathrm{x} x$ & $\mathrm{x} x$ & $\mathrm{x} x$ & $\mathrm{x} x$ & $\mathrm{x} x$ & $\mathrm{x} x$ \\
\hline bTag_Tagga_HF & $\mathrm{x} x$ & $\mathrm{x} x$ & $\mathrm{x} x$ & $\mathrm{x} x$ & $\mathrm{x} x$ & $\mathrm{x} x$ & $\mathrm{x} \mathrm{x}$ & $\mathrm{x} \mathrm{x}$ \\
\hline bTag_Tagga_LF_WH & $\mathrm{x} x$ & $\mathrm{x} x$ & $\mathrm{x} x$ & $\mathrm{x} x$ & $\mathrm{x} x$ & $\mathrm{x} x$ & $\mathrm{x} x$ & $\mathrm{x} x$ \\
\hline EMID & $\mathrm{x} x$ & $\mathrm{x} x$ & $\mathrm{x} x$ & $\mathrm{x} x$ & & & & \\
\hline Bkgd_Xsec_EW & $\mathrm{x} x$ & $\mathrm{x} x$ & $\mathrm{x} x$ & $\mathrm{x} x$ & $\mathrm{x} \mathrm{x}$ & $\mathrm{x} \mathrm{x}$ & $\mathrm{x} \mathrm{x}$ & $\mathrm{x} \mathrm{x}$ \\
\hline Jet_NN_MMLM & $\mathrm{x} x$ & $\mathrm{x} x$ & & & $\mathrm{x} x$ & $\mathrm{x} x$ & & \\
\hline Bkgd_Xsec_NN_HF_WH & $\mathrm{x}$ & $\mathrm{x}$ & & & $\mathrm{x}$ & $\mathrm{x}$ & & \\
\hline Bkgd_WbbRW_NN_shape & $\mathrm{x} x$ & $\mathrm{x} x$ & & & $\mathrm{x} x$ & $\mathrm{x} x$ & & \\
\hline Bkgd_QCDev & $\mathrm{xx}$ & $\mathrm{x} x$ & $\mathrm{xx}$ & $\mathrm{x} \mathrm{x}$ & & & & \\
\hline Bkgd_WjjRW_NN_shape & $\mathrm{x} x$ & $\mathrm{x} x$ & & & $\mathrm{x} \mathrm{x}$ & $\mathrm{xx}$ & & \\
\hline Bkgd_Xsec_Top & $\mathrm{xx}$ & $\mathrm{x} x$ & $\mathrm{x} \mathrm{x}$ & $\mathrm{x} x$ & $\mathrm{x} x$ & $\mathrm{x} x$ & $\mathrm{x} \mathrm{x}$ & $\mathrm{x} \mathrm{x}$ \\
\hline Bkgd_Xsec_singletop & $\mathrm{x} \mathrm{x}$ & $\mathrm{x} x$ & $\mathrm{x} x$ & $\mathrm{x} x$ & $\mathrm{x} x$ & $\mathrm{x} x$ & $\mathrm{x} x$ & $\mathrm{x} x$ \\
\hline Jet_DJ_MMLM & & & $\mathrm{x} x$ & $\mathrm{x} x$ & & & $\mathrm{x} x$ & $\mathrm{x} x$ \\
\hline Bkgd_Xsec_DJ_HF_WH & & & $\mathrm{x}$ & $\mathrm{x}$ & & & $\mathrm{x}$ & $\mathrm{x}$ \\
\hline Bkgd_WbbRW_DJ_shape & & & $\mathrm{x} x$ & $\mathrm{x} x$ & & & $\mathrm{x} x$ & $\mathrm{x} x$ \\
\hline Bkgd_WjjRW_DJ_shape & & & $\mathrm{x} x$ & $\mathrm{x} x$ & & & $\mathrm{x} x$ & $\mathrm{x} x$ \\
\hline Bkgd_Xsec_NN_HF_WH_IIb & $\mathrm{x}$ & $\mathrm{x}$ & & & $\mathrm{x}$ & $\mathrm{x}$ & & \\
\hline Bkgd_Xsec_DJ_HF_WH_IIb & & & $\mathrm{x}$ & $\mathrm{x}$ & & & $\mathrm{x}$ & $\mathrm{x}$ \\
\hline MUTrigger_WH & & & & & $\mathrm{x} \mathrm{x}$ & $\mathrm{x} \mathrm{x}$ & $\mathrm{x} x$ & $\mathrm{xx}$ \\
\hline MUID & & & & & $\mathrm{x} x$ & $\mathrm{x} x$ & $\mathrm{x} x$ & $\mathrm{x} x$ \\
\hline Bkgd_QCDmv & & & & & $\mathrm{x} x$ & $\mathrm{x} x$ & $\mathrm{x} x$ & $\mathrm{x} x$ \\
\hline WHNLO & $\mathrm{x} x$ & $\mathrm{xx}$ & $\mathrm{x} \mathrm{x}$ & $\mathrm{x} \mathrm{x}$ & $\mathrm{x} x$ & $\mathrm{x} x$ & $\mathrm{x} x$ & $\mathrm{x} x$ \\
\hline
\end{tabular}

Table 7.1: Sources of the systematic uncertainties of the sixteen event samples. The naming conventions is explained in the Table 7.2. The terms labeled with $\times$ means they are associated. Those sources with names starting with Bkgd apply only to the background, while the other apply to both the background and signal, except for the last error of the table is only for $W H$. 


\begin{tabular}{ll}
\hline \hline Collie name & Explanation, see following tables for the processes to which they apply \\
\hline \hline DZero_Lumi & Part of the Luminosity uncertainty coming from Dzero \\
Lumi & Part of the Luminosity uncertainty correlated with CDF \\
JESID & JES, JER and Jet-id uncertainties added in quadrature \\
bTag_Tagga_HF & Tagging and Taggability uncertainties added in quadrature, for heavy flavor \\
bTag_Tagga_LF_WH & Tagging and Taggability uncertainties added in quadrature, for direct light tagging \\
EMID & EM-id, -reconstruction, -scale, -smearing and -trigger added in quadrature \\
Bkgd_Xsec_EW & Diboson cross section uncertainties \\
Jet_NN_MMLM & "additional" dijet mass shape uncertainty for 2 jet sample \\
Bkgd_Xsec_NN_HF_WH & uncertainty on Heavy flavor K-factor in Run IIa for 2 jet sample \\
Bkgd_WbbRW_NN_shape & uncertainty on Wbb reweighting for 2 jet sample \\
Bkgd_QCDev & uncertainty on electron QCD background, annticorrelated with Wjj normalization \\
Bkgd_WjjRW_NN_shape & uncertainty on Wjj reweighting for 2 jet sample \\
Bkgd_Xsec_Top & $t \bar{t}$ cross section uncertainty \\
Bkgd_Xsec_singletop & single top cross section uncertainty \\
Jet_DJ_MMLM & "additional" dijet mass shape uncertainty for 3 jet sample \\
Bkgd_Xsec_DJ_HF_WH & uncertainty on Heavy flavor K-factor in Run IIa for 3 jet sample \\
Bkgd_WbbRW_DJ_shape & uncertainty on Wbb reweighting for 3 jet sample \\
Bkgd_WjjRW_DJ_shape & uncertainty on Wjj reweighting for 3 jet sample \\
Bkgd_Xsec_NN_HF_WH_RunIIb & uncertainty on Heavy flavor K-factor in Run IIb for 2 jet sample \\
Bkgd_Xsec_DJ_HF_WH_RunIIb & uncertainty on Heavy flavor K-factor in Run IIb for 3 jet sample \\
MUTrigger_WH & uncertainty on muon trigger efficiency \\
MUID & muon-id, -reconstruction, and -smearing added in quadrature \\
Bkgd_QCDmv & uncertainty on muon QCD background, annticorrelated with Wjj normalization \\
WHNLO & uncertainty on $W H$ higher order kinematics (applies only to the signal) \\
\hline \hline
\end{tabular}

Table 7.2: The naming convention of the systematic uncertainties in Tab. 7.1. Those sources with names starting with $B k g d$ apply only to the background, while the other apply to both the background and signal, except for the last entry of the table is only for $W H$. 


\begin{tabular}{|c|c|c|c|c|c|c|c|}
\hline & $\overline{\mathrm{WH}}$ & $\overline{\mathrm{WW} / \mathrm{WZ} / \mathrm{ZZ}}$ & $\overline{\mathrm{Wbb} / \mathrm{Wcc}}$ & $\overline{\mathrm{Wjj} / W c j}$ & $\overline{t \bar{t}}$ & $\mathrm{tb} / \mathrm{tqb}$ & multijet \\
\hline EM Trigger eff. & 2.9 & 4.0 & 3.0 & 3.0 & 3.1 & 4.1 & \\
\hline EM ID/Reco eff. & 4.2 & 4.2 & 4.2 & 4.2 & 4.2 & 4.2 & \\
\hline EM energy/smearing & 3.0 & 3.0 & 3.0 & 3.0 & 3.0 & 3.0 & \\
\hline Jet ID/Reco eff. & 3.0 & 3.0 & 3.0 & 3.0 & 3.0 & 3.0 & \\
\hline Jet Energy Scale & 2.0 & 5.0 & 5.0 & 2.0 & 3.5 & 5.0 & \\
\hline Jet SSR & 2.0 & 6.0 & 4.0 & 3.0 & 5.0 & 2.0 & \\
\hline ALPGEN reweighting(shape) & & & 5.0 & 5.0 & & & \\
\hline Dijet mass (shape) & & & 7.0 & 7.0 & & & \\
\hline Jet taggability & 3.0 & 3.0 & 3.0 & 3.0 & 3.0 & 3.0 & \\
\hline NN-tagger S.F. & 5.0 & 5.0 & 6.0 & 15.0 & 1.0 & 1.5 & \\
\hline Cross Section & 6.0 & 6.0 & & & 10.0 & 12.0 & \\
\hline Normalization & & & 20.0 & 5.0 & & & \\
\hline Total uncertainty & 13.1 & 15.5 & 25.0 & 20.3 & 17.4 & 17.4 & 26.0 \\
\hline
\end{tabular}

Table 7.3: Systematic uncertainties (\%) for different physics processes containing electron in the final states with single jet b-tagged. The total uncertainties are also summarized in the table.

\begin{tabular}{|c|c|c|c|c|c|c|c|}
\hline & $\overline{\mathrm{WH}}$ & $\overline{\mathrm{WW}} / \mathrm{WZ} / \mathrm{ZZ}$ & $\overline{\mathrm{Wbb} / \mathrm{Wcc}}$ & $\overline{\mathrm{Wjj} / \mathrm{Wcj}}$ & $\bar{t} t \bar{t}$ & tb/tqb & multijet \\
\hline EM Trigger eff. & 3.6 & 4.0 & 3.0 & 3.0 & 3.7 & 4.5 & \\
\hline EM ID/Reco eff. & 4.2 & 4.2 & 4.2 & 4.2 & 4.2 & 4.2 & \\
\hline EM energy/smearing & 3.0 & 3.0 & 3.0 & 3.0 & 3.0 & 3.0 & \\
\hline Jet ID/Reco eff. & 3.0 & 3.0 & 3.0 & 3.0 & 3.0 & 3.0 & \\
\hline Jet Energy Scale & 2.0 & 3.5 & 3.0 & 2.0 & 3.0 & 3.0 & \\
\hline Jet SSR & 2.0 & 3.0 & 6.0 & 2.0 & 5.0 & 2.0 & \\
\hline ALPGEN reweighting(shape) & & & 5.0 & 5.0 & & & \\
\hline Dijet mass (shape) & & & 7.0 & 7.0 & & & \\
\hline Jet taggability & 3.0 & 3.0 & 3.0 & 3.0 & 3.0 & 3.0 & \\
\hline NN-tagger S.F. & 6.0 & 8.0 & 10.0 & 25.0 & 6.5 & 6.5 & \\
\hline Cross Section & 6.0 & 6.0 & & & 10.0 & 12.0 & \\
\hline Normalization & & & 20.0 & 5.0 & & & \\
\hline Total uncertainty & 14.9 & 15.8 & 26.4 & 28.4 & 17.4 & 17.7 & 26.0 \\
\hline
\end{tabular}

Table 7.4: Systematic uncertainties (\%) for different physics processes containing electron in the final states with double jet b-tagged. The total uncertainties are also summarized in the table. 


\begin{tabular}{|c|c|c|c|c|c|c|c|}
\hline & $\overline{\mathrm{WH}}$ & $\overline{\mathrm{WW} / \mathrm{WZ} / \mathrm{ZZ}}$ & $\overline{\mathrm{Wbb} / \mathrm{Wcc}}$ & Wjj/Wcj & $\overline{t t \bar{t}}$ & $\mathrm{tb} / \mathrm{tqb}$ & multijet \\
\hline Muon Trigger eff. & 5.0 & 5.0 & 5.0 & 5.0 & 5.0 & 5.0 & \\
\hline Muon (iso.) ID/Reco eff. & 3.0 & 3.0 & 3.0 & 3.0 & 3.0 & 3.0 & \\
\hline Muon track match eff. SF & 2.0 & 2.0 & 2.0 & 2.0 & 2.0 & 2.0 & \\
\hline Muon energy/smearing & 2.0 & 2.0 & 2.0 & 2.0 & 2.0 & 2.0 & \\
\hline Jet ID/Reco eff. & 3.0 & 3.0 & 3.0 & 3.0 & 3.0 & 3.0 & \\
\hline Jet Energy Scale & 2.0 & 5.0 & 5.0 & 2.0 & 3.5 & 5.0 & \\
\hline Jet SSR & 2.0 & 6.0 & 4.0 & 3.0 & 5.0 & 2.0 & \\
\hline ALPGEN reweighting(shape) & & & 5.0 & 5.0 & & & \\
\hline Dijet mass (shape) & & & 7.0 & 7.0 & & & \\
\hline Jet taggability & 3.0 & 3.0 & 3.0 & 3.0 & 3.0 & 3.0 & \\
\hline NN-tagger S.F. & 5.0 & 5.0 & 6.0 & 15.0 & 1.0 & 1.5 & \\
\hline Cross Section & 6.0 & 6.0 & & & 10.0 & 12.0 & \\
\hline Normalization & & & 20.0 & 5.0 & & & \\
\hline Total uncertainty & 13.9 & 15.8 & 25.5 & 20.9 & 17.4 & 17.7 & 26.0 \\
\hline
\end{tabular}

Table 7.5: Systematic uncertainties (\%) for different physics processes containing muon in the final states with single jet b-tagged. The total uncertainties are also summarized in the table.

\begin{tabular}{|c|c|c|c|c|c|c|c|}
\hline & $\overline{\mathrm{WH}}$ & $\overline{\mathrm{WW} / \mathrm{WZ} / \mathrm{ZZ}}$ & $\overline{\mathrm{Wbb} / \mathrm{Wcc}}$ & $\overline{\mathrm{Wjj} / \mathrm{Wcj}}$ & $t \bar{t}$ & tb/tqb & multijet \\
\hline Muon Trigger eff. & 5.0 & 5.0 & 5.0 & 5.0 & 5.0 & 5.0 & \\
\hline Muon (iso.) ID/Reco eff. & 3.0 & 3.0 & 3.0 & 3.0 & 3.0 & 3.0 & \\
\hline Muon track match eff. SF & 2.0 & 2.0 & 2.0 & 2.0 & 2.0 & 2.0 & \\
\hline Muon energy/smearing & 2.0 & 2.0 & 2.0 & 2.0 & 2.0 & 2.0 & \\
\hline Jet ID/Reco eff. & 3.0 & 3.0 & 3.0 & 3.0 & 3.0 & 3.0 & \\
\hline Jet Energy Scale & 2.0 & 3.5 & 3.0 & 2.0 & 3.0 & 3.0 & \\
\hline Jet SSR & 2.0 & 3.0 & 6.0 & 2.0 & 5.0 & 2.0 & \\
\hline ALPGEN reweighting(shape) & & & 5.0 & 5.0 & & & \\
\hline Dijet mass (shape) & & & 7.0 & 7.0 & & & \\
\hline Jet taggability & 3.0 & 3.0 & 3.0 & 3.0 & 3.0 & 3.0 & \\
\hline NN-tagger S.F. & 6.0 & 8.0 & 10.0 & 25.0 & 6.5 & 6.5 & \\
\hline Cross Section & 6.0 & 6.0 & & & 10.0 & 12.0 & \\
\hline Normalization & & & 20.0 & 5.0 & & & \\
\hline Total uncertainty & 15.3 & 16.0 & 26.8 & 28.8 & 17.4 & 17.7 & 26.0 \\
\hline
\end{tabular}

Table 7.6: Systematic uncertainties (\%) for different physics processes containing muon in the final states with double jet b-tagged. The total uncertainties are also summarized in the table. 
Higgs boson signal over the background events. Therefore, using the neural network output of $W+2$ jet events for both the data and simulated samples, the limits on the production cross sections of $p \bar{p} \rightarrow W H \rightarrow l \nu b \bar{b}$ are calculated through a modified frequentist method with Poisson log-likelihood test [116] [117].

\subsubsection{The Limit Calculation Method}

The so called $C L_{s}$ method [116] provides an efficient procedure for combining independent results with small statistics to test the hypotheses of the Higgs boson's existence at a given mass. The key features of the method are the modified frequentist confidence level $C L_{s}$ and the Poisson log-likelihood ratio test statistic.

Given that for a single bin $i$, the predicted signal and background numbers are $s_{i}$ and $b_{i}$, and the observed number in the data is $d_{i}$, the ratio of Poisson likelihoods can be built as

$$
Q_{i}=\frac{P(\text { data } \mid s+b)}{P(\text { data } \mid b)}=\frac{e^{-\left(s_{i}+b_{i}\right)}\left(s_{i}+b_{i}\right)^{d_{i}}}{d_{i} !} / \frac{e^{-b_{i}} b_{i}^{d_{i}}}{d_{i} !}
$$

The total $\mathrm{N}$ bins can thus be combined into single ratio

$$
Q=\prod_{i=1}^{N} Q_{i}
$$

In this way, the distributions of different event samples, such as single and double btagged samples, are able to be combined together straightforwardly. An alternative form called log-likelihood ratio (LLR) is introduced to simplify the computation, as

$$
\chi_{d}=\sum_{i=1}^{N} \chi_{d_{i}}=\sum_{i=1}^{N}-2 \ln Q_{i}=\sum_{i=1}^{N} 2\left[s_{i}-d_{i} \ln \left(1+\frac{s_{i}}{b_{i}}\right)\right] .
$$

Note that there is singularity in the test statistic in case of zero background expectation $b_{i}=0$. 
The frequentist confidence level for excluding the signal+background $(s+b)$ hypothesis is

$$
C L_{s+b}=P_{s+b}\left(\chi \leq \chi_{d}\right)=\int_{-\infty}^{\chi_{d}} \frac{d P_{s+b}}{d \chi} d \chi .
$$

However, the use of $C L_{s+b}$ alone may even lead to exclusion of the background, if there are few background events observed. The confidence level is modified to be

$$
C L_{s}=\frac{C L_{s+b}}{C L_{b}}
$$

where $C L_{b}=P_{b}\left(\chi \leq \chi_{d}\right)=\int_{-\infty}^{\chi_{d}} \frac{d P_{b}}{d \chi} d \chi$. The Higgs signal is excluded at a confidence level of $1-C L_{s}$. The $d P_{s+b} / d \chi$ and $d P_{b} / d \chi$ are defined as the distributions of Poisson probability with a mean value of $s+b$ or $b$. The distributions could be sampled via many pseudo-experiments simulating the possible outcomes. The computation time is shortened by instead computing the Poisson probability distribution function (PDF) for each instance of the test statistic and convoluting these PDFs.

The systematic uncertainties are incorporated by smearing the PDFs of $P_{s+b}$ and $P_{b}$ with Gaussian functions. In practice, for each pseudo-experiment, the mean value of the PDF is recalculated as

$$
\mu_{\text {new }}=\mu\left(1+\sum_{k} G_{k}\right)
$$

where $G_{k}$ is the random value from an individual Gaussian distribution of uncertainty source $k$. The other practical method is to incorporate uncertainties via a Gaussian approximation into the explicit calculation of PDFs. More details can be found in [117]. The correlated uncertainty must be maintained the same among different event samples for each pseudo-experiment. The above procedures have the effect of broadening the PDFs and degrading the separation power. A so called profilelikelihood technique [118] is introduced to reduce the impact by maximizing the 
background PDFs over the possible space to find the model that best fits the data. Note that in order to speed up computation and reduce the uncertainty due to histogram shape, the histograms of the final variables are rebinned and smoothed via the $353 Q H$ algorithm [119] and the Gaussian adaptive kernel estimation algorithm [120].

The limits on the production cross section in this analysis are quoted at 95\% C.L. $\left(C L_{s}=5 \%\right)$. The production rate of $W H$ is $R_{W H}=\sigma_{W H} \times \mathcal{L} \times \epsilon_{\text {acceptance }}$, where

$\sigma_{W H}, \mathcal{L}$ and $\epsilon_{\text {acceptance }}$ are the cross section, luminosity and signal acceptance. If the criteria that $C L_{s} \leq 5 \%$ is not achieved, the cross section is scaled up by a ratio $X_{f}$ to recalculate the limits. This is done iteratively by adjusting the $X_{f}$ up step by step until the $C L_{s} \leq 5 \%$ is satisfied. Two values of $C L_{s}$, expected and observed, are used in the analysis. The expected limits are calculated simply by substituting $d$ with $b$ in Eq. 7.5.

\subsubsection{The Upper Limits on $W H$ Production Cross Section}

The limits on the production cross section of $\sigma(p \bar{p} \rightarrow W H) \times B R(H \rightarrow b \bar{b})$ are calculated for a total of 16 event samples according to combinations of Run IIa and Run IIb, electron and muon, single and double b-tagged jets, as well as $W+2 j e t$ and $W+3 j e t$. Note that since neural network is trained against only $W b b$ background, the dijet mass is used instead of the $\mathrm{NN}$ output in the limit calculation for $W+3 j$ et event samples. The mass of the Higgs boson is searched in the range of $100-150$ $\mathrm{GeV}$ with each mass point separated by $5 \mathrm{GeV}$.

The ratios of the cross section limits to the Standard Model prediction for $m_{H}=$ $115 \mathrm{GeV}$ are shown in Tab. 7.7. Compared to the previous analysis on $1 \mathrm{fb}^{-1}$ of data from the Run IIa dataset, the expected (observed) limit improves by $\sim 14 \%(43 \%)$ based from the event selection. Both the individual uses of the Neural Network 


\begin{tabular}{|l|c|c|c|c|}
\hline Analysis & Dijet Mass & Neural Network & Matrix Element & ME + NN \\
\hline \hline $1.0 \mathrm{fb}^{-1}$ Run IIa results (March 2007) & $14.2(18.8)$ & $-(-)$ & $-(-)$ & $-(-)$ \\
\hline \hline Run IIa 2jet & $12.4(10.7)$ & $10.9(10.8)$ & $10.6(10.1)$ & $10.5(10.1)$ \\
Run IIa W+2/3jet & $11.8(12.2)$ & $10.5(11.7)$ & $10.1(10.9)$ & $10.0(10.6)$ \\
Run IIb W+2jet & $10.3(13.0)$ & $8.9(9.0)$ & $8.3(7.8)$ & $8.1(8.7)$ \\
Run IIb W+2/3jet & $9.7(11.3)$ & $8.7(8.2)$ & $8.1(7.3)$ & $7.8(7.6)$ \\
Run IIa/b W+2jet & $7.9(8.8)$ & $7.2(7.3)$ & $6.6(6.3)$ & $6.6(7.4)$ \\
\hline Run IIa/b W+2/3jet & $7.5(8.5)$ & $6.9(7.1)$ & $6.4(6.1)$ & $6.4(6.7)$ \\
\hline
\end{tabular}

Table 7.7: The ratios of the expected (observed) production cross section limits to the Standard Model prediction, where the cross section is $\sigma(p \bar{p} \rightarrow W H) \times B R(H \rightarrow b \bar{b})$ with the Higgs boson mass $m_{H}=115 \mathrm{GeV}$. The electron and muon, single and double b-tagged have been combined for all the numbers in the table. The $1.0 \mathrm{fb}^{-1}$ Run IIa results (March 2007) are the previous cut-based analysis without multivariate analysis techniques.

and the Matrix Element methods significantly enhance the sensitivity of the analysis to the Higgs boson signal. Combining the Matrix Element and Neural Network approaches further lowers the expected (observed) cross section limit from $7.5(8.5)$ to $6.4(6.7)$, corresponding to an improvement of at least $15 \%$ relative to the cutbased only analysis.

The sensitivity of this analysis to the Higgs boson signal is interpreted via the distributions of log-likelihood ratios (LLR), as shown in Fig. 7.1(a). The capability of discriminating the signal+background $(\mathrm{s}+\mathrm{b})$ hypothesis from the background only (b) hypotheses is reflected by the separation between $L L R_{s+b}$ and $L L R_{b}$ curves. The fluctuation of the background only hypothesis, including the systematic uncertainty, is represented by the bands of the $L L R_{b}$, of which the width for $1 \sigma$ and $2 \sigma$ is shown in the plot. The distribution of $L L R_{o b s}$ with respect to $L L R_{s+b}$ and $L L R_{b}$ indicates how much the data distribution is like signal+background or background only. The significance of the $L L R_{o b s}$ in this analysis is still limited by statistics, within $1 \sigma$ standard deviation.

The cross section limits on $\sigma(p \bar{p} \rightarrow W H) \times B R(H \rightarrow b \bar{b})$ with $m_{H}$ range 100 - $150 \mathrm{GeV}$ are summarized in Tab. 7.8 and Fig. 7.1(b). The observed limits are 
consistent with the expected ones. The ratios of the cross sections limits increase with the Higgs boson mass, as expected from the discussions in Section 2.2.2. The cross section limits are at least 4 times above the prediction of the Standard Model theory. The observed (expected) ratio for the Higgs boson with a mass of $115 \mathrm{GeV}$ is $6.7(6.4)$, which translates to a cross section of $\sim 0.8$ pb compared to the Standard Model expectation of $0.13 \mathrm{pb}$.

\begin{tabular}{|l|c|c|c|c|c|c|c|c|c|c|c|}
\hline$m_{H}(\mathrm{GeV})$ & 100 & 105 & 110 & 115 & 120 & 125 & 130 & 135 & 140 & 145 & 150 \\
\hline \hline$\sigma_{\text {expected }} / \sigma_{S M}$ & 5.3 & 4.9 & 5.8 & 6.4 & 7.5 & 9.5 & 13.7 & 16.1 & 23.0 & 36.1 & 56.0 \\
\hline$\sigma_{\text {observed }} / \sigma_{S M}$ & 5.2 & 4.2 & 5.1 & 6.7 & 8.2 & 9.8 & 16.7 & 17.3 & 23.3 & 43.7 & 52.4 \\
\hline
\end{tabular}

Table 7.8: The ratios of $95 \%$ C.L. expected and observed limits on $\sigma(p \bar{p} \rightarrow W H) \times B(H \rightarrow b \bar{b})$, to their corresponding Standard Model prediction, as a function of the Higgs boson mass $m_{H}$.

\subsection{Conclusions}

A search for $p \bar{p} \rightarrow W H \rightarrow l \nu b \bar{b}$ has been performed with $2.7 \mathrm{fb}^{-1}$ of data collected at the DØ detector between April 2002 and April 2008. The dataset is split into Run IIa and Run IIb, with Run IIb utilizing an upgrade of the DØ detector in 2006 with better tracking and triggers.

To select events of the Higgs boson associated with a $W$ boson, the events are first required to fire specific $\mathrm{D} \varnothing$ triggers. Those events with an isolated high $p_{T}$ electron or muon, a high missing $E_{T}$ and 2 or 3 energetic jets in the final state, are selected into different event samples according to lepton types and number of jets. The DØ neural network b-jet tagging algorithm is used to further select events into orthogonal event samples, one with exactly one jet tagged as a Tight $b$ jet and the other with two jets tagged as oldLoose $b$ jets. The kinematic distributions and number of events in the data samples are consistent with the simulated events of the 
Standard Model processes.

We explored multivariate analysis techniques to improve the Higgs signal sensitivity. The Matrix Element method correlates the parton level calculation of physics processes with all available kinematic information in the event to discriminate signal from background. The Matrix Element discriminant, as well as seven relevant variables, are further used as the input to a neural network to better separate the Higgs signal.

No evidence of the Higgs boson is observed. Instead, upper limits on the Higgs production cross section are calculated using the modified frequentist $C L_{s}$ method. We present the limits in the form of a ratio of the cross section limit $\sigma(p \bar{p} \rightarrow W H) \times$ $B R(H \rightarrow b \bar{b})$ to the Standard Model expectation; the observed (expected) limit for the Higgs boson with $m_{H}=115 \mathrm{GeV}$ is 6.7 (6.4). The sensitivity of this analysis has improved relative to the previous analysis due to the improved event selection and multivariate techniques. The results of the $W H$ search with $m_{H}$ ranging from $100 \mathrm{GeV}$ to $150 \mathrm{GeV}$, significantly contribute to the $0.9-4.2 \mathrm{fb}^{-1}$ results on the Standard Model Higgs production in March 2009. The D $\varnothing$ combination results are shown in Fig. 7.2 [19], and the Tevatron combination results are shown in Fig. 7.3 $[20]$.

The sensitivity of the $W H$ search in the future could be further improved in many ways, some of which are work in progress:

- Integrated luminosity of the data. So far $6.1 \mathrm{fb}^{-1}$ of data has been collected at the DØ detector till up to June 2009, of which $5 \mathrm{fb}^{-1}$ is being analyzed. Significantly more data will be delivered by the Tevatron through the year 2010. The statistics might be sufficient for an exclusion or evidence on the low mass Higgs boson. 
- More triggers could be added for events with electron in the final states. In this analysis, Single EM and EM+JET triggers are applied on Run IIa dataset, while only Single EM triggers are used for Run IIb dataset. For future analysis, Single EM and EM+JET triggers or even more could be used through all the dataset. This would bring at least $\sim 5 \%$ increase to the event acceptance.

- The muon isolation criteria of ScaleCalorimeter Halo $<0.08$ and ScaledTrackHalo $<$ 0.06 could be replaced with Calorimeter Halo $<2.5 \mathrm{GeV}$ and TrackHalo $<2.5$ $\mathrm{GeV}$, in order to increase the acceptance to the muons particularly for those with $p_{T}<20 \mathrm{GeV}$. The other lepton identification criteria could also be further optimized. The requirement on the Missing energy $\mathscr{H}_{T}>20 \mathrm{GeV}$ could be loosened to $\mathscr{H}_{T}>15 \mathrm{GeV}$, which is feasible with muon in the final state and increases the signal acceptance by a $\sim 5 \%$. In addition, more soft interaction background events (>10\%) could be rejected by requiring jets to be confirmed with a vertex.

- An improvement on the dijet mass resolution will dramatically improve the sensitivity of this analysis. It has been seen in the $Z H$ analysis via jet energy resolution, but not yet confirmed with $W H$ analysis.

- Improvements on the b-tagging efficiency would most efficiently increase the sensitivity of the analysis. A smaller fake rate would also greatly reduce the systematic uncertainties. Asymmetric b-tagging, where the tagging criteria of double b-tagged jets could be different on the leading jet and sub-leading jet, could also be explored.

- The demanding computation time of the Matrix Element method could be challenging to an analysis with higher integrated luminosity. Some other multivari- 
ate analysis techniques can be explored. The Boosted Decision Tree (BDT) is an ideal candidate method because it is fast, stable, less sensitive to additional variables and can easily achieve its best performance.

With increasing capability of the Tevatron and LHC, a conclusion on the Higgs boson will become a reality in the near future. 


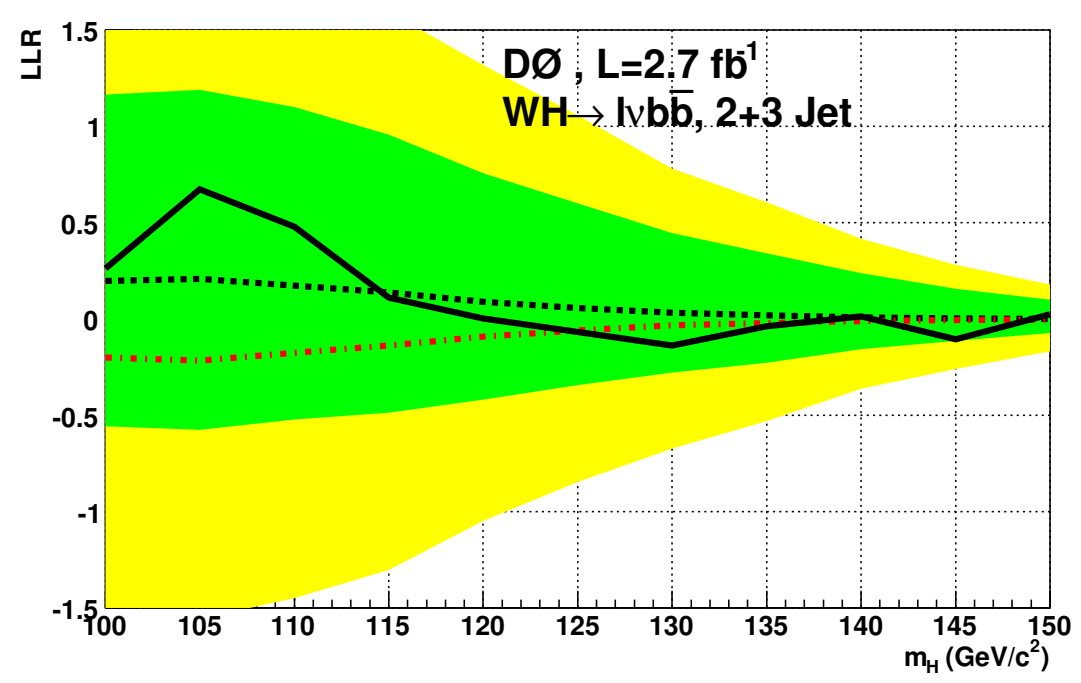

(a) Log-likelihood ratios versus $m_{H}$

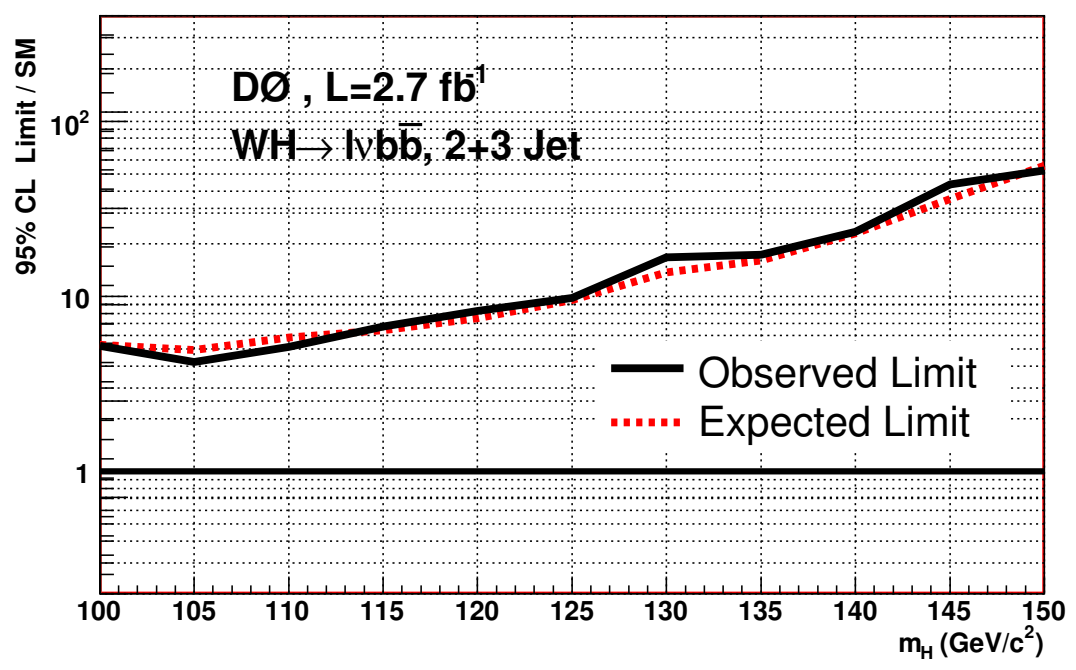

(b) The ratios of the cross section limits versus $m_{H}$

Figure 7.1: The results of calculation on the upper limits of $W H$ production cross section $\sigma(p \bar{p} \rightarrow$ $W H) \times B R(H \rightarrow b \bar{b})$. The $2.7 \mathrm{fb}^{-1}$ of Run IIa and RunIIb datasets with the 16 event sub-samples are combined in the plots. a) The log-likelihood ratios as a function of the Higgs boson mass $m_{H}$. The dashed lines represent for $L L R_{b}$ (upper line) and $L L R_{s+b}$ (lower line), while the solid line represents $L L R_{o b s}$. The green band (inner region) and yellow band region (extended from the inner to the outermost) stand for the $\pm 1 \sigma$ and $\pm 2 \sigma$ standard deviations. b) The ratios of the upper limits on the production cross sections to those predicted by the Standard Model for $\sigma(p \bar{p} \rightarrow W H) \times B R(H \rightarrow b \bar{b})$, as a function of $m_{H}$. The solid line and dashed lines represent the observed and expected limits at $95 \%$ C.L. respectively. The straight line at ratio $=1.0$ indicates the Standard Model prediction. 


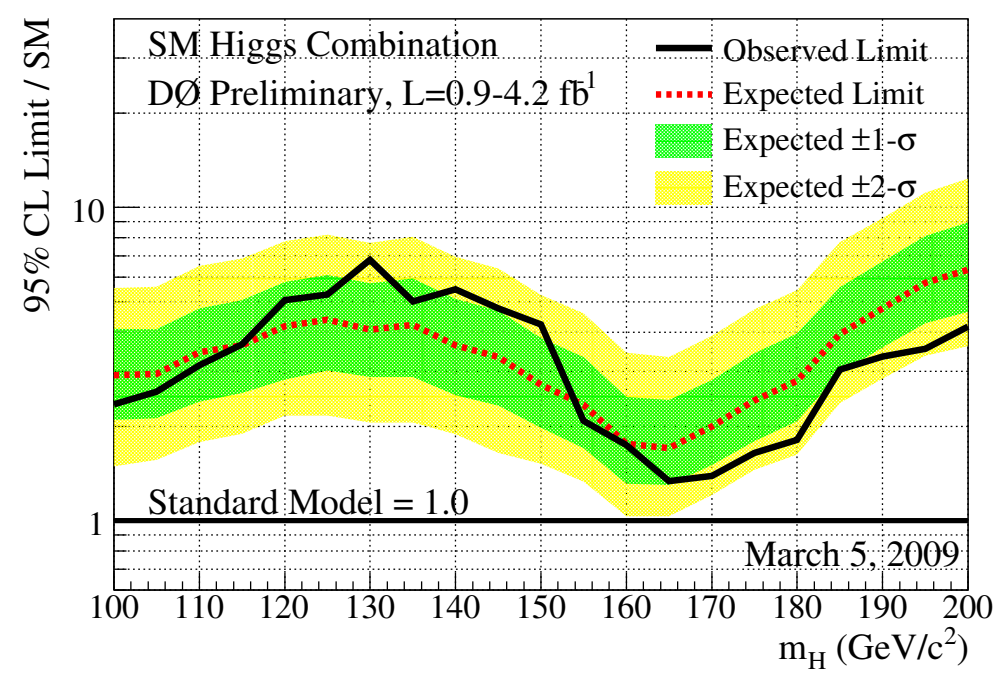

Figure 7.2: The ratios of 95\%C.L. cross section limit on the Standard Model Higgs production to the Standard Model expectation, as a function of $m_{H}$. The solid and dashed lines represent the observed and expected limits respectively, while the green and yellow bands stand for $1 \sigma$ and $2 \sigma$ standard deviations. The searches for $W H / Z H / H$, with $H \rightarrow b \bar{b} / W^{+} W^{-} / \gamma \gamma / \tau^{+} \tau^{-}$final states, using $0.9-4.2 \mathrm{fb}^{-1}$ of data collected at the $\mathrm{D} \varnothing$ detector have been combined in this plot. [19]

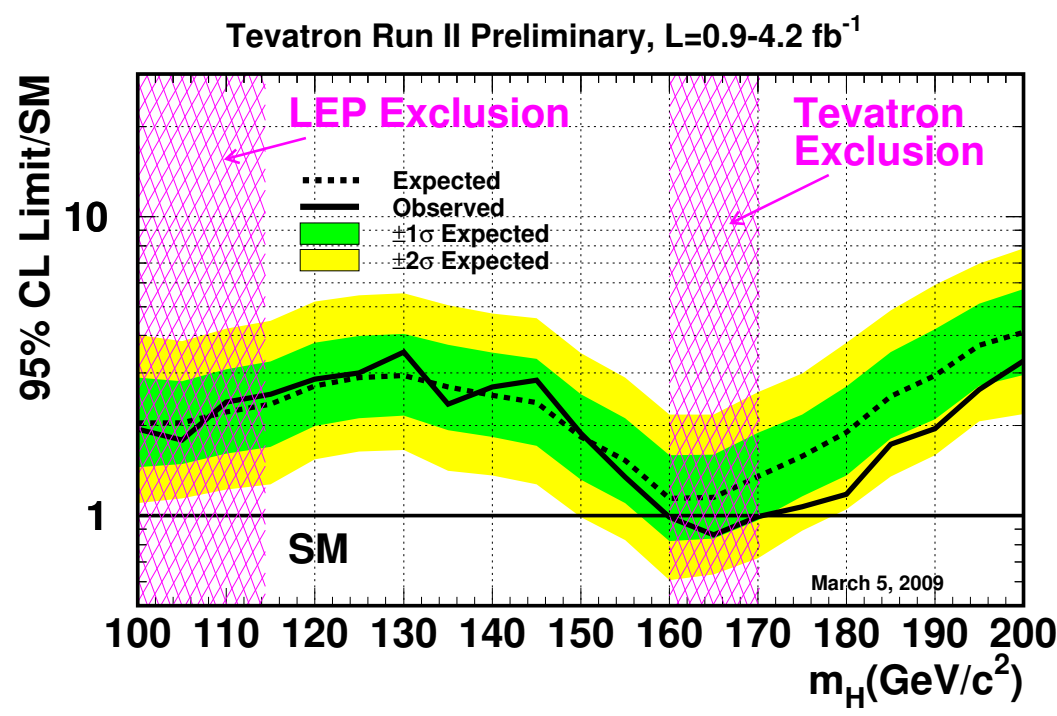

Figure 7.3: Observed and expected upper limits on the Standard Model Higgs production at the Tevatron as a function of the Higgs boson mass. The limits are expressed as the constraints on the ratio of experimental production cross section to the one predicted by the Standard Model. The solid curve represents the observed ratio, while the dotted is the expected (background-only hypothesis) ratio. The limits are calculated with a Bayesian method at $95 \%$ C.L.. The shaded bands indicate fluctuation regions of the limits at $68 \%$ and $95 \%$ probability respectively in the absence of signal. [20] 
BIBLIOGRAPHY 


\section{BIBLIOGRAPHY}

[1] A. Djouadi. The Anatomy of Electroweak Symmetry Breaking - Tome I: The Higgs Boson in the Standard Model. hep-ph/0503172, 1964.

[2] T. Hambye and K. Riesselmann. Phys. Rev., D55:7255, 1997.

[3] LEP Electroweak Working Group. http://lepewwg.web.cern.ch/LEPEWWG/.

[4] CDF and D0 Collaborations. Results of the Tevatron Higgs Sensitivity Study. FERMILABPUB-03/320-E, 2003.

[5] A. Djouadi, J. Kalinowski, and M. Spira. HDECAY: a Program for Higgs Boson Decays in the Standard Model and its Supersymmetric Extension. hep-ph/9704448, 1997.

[6] M. Sanders. http://www-d0.fnal.gov/〜msanders/Higgs/CSTimesBr.html.

[7] http://www-bdnew.fnal.gov/operations.html.

[8] C.W. Schmidt. Review of Negative Hydrogen Ion Sources. Linear Accelerator Conference, Albuquerque, NM, Sep. 1990.

[9] J. Thompson. Introduction to Colliding Beams at Fermilab. FERMILAB-TM-1909, 1994.

[10] http://www-bdnew.fnal.gov/operations/rookie_books/rbooks.html.

[11] V. Abazov et al. The Upgraded DØ Detector. Nucl. Instrum. Meth., A565:463, 2006.

[12] T. Edwards et al. Determination of the Effective Inelastic ppbar Cross-Section for the DØ Run II Luminosity Measurement. FERMILAB-TM-2278-E, 2004.

[13] D0 Vertexing Group. http://www-do.fnal.gov/global_tracking/vertex. html\#vertexing_tasks.

[14] A. Kumar et al. Electron Likelihood Study. Do Internal Note 4769, 2005.

[15] G. Blazey et al. Run II Jet Physics. hep-ex/0005012, 2000.

[16] A. Harel and J. Kvita. p20 JetID Efficiencies and Scale Factors. D0 Internal Note 5634, 2008.

[17] P. Calfayan et al. Muon Identification Certification for p17 data. D0 Internal Note 5157, 2007.

[18] T. Gadfort et al. Performance of the D $\varnothing \mathrm{NN}$ b-tagging Tool on p20 Data. D0 Internal Note $5554,2008$.

[19] The D0 Collaboration. Combined Upper Limits on Standard Model Higgs Boson Production from the D0 Experiment in $0.9-4.2 \mathrm{fb}^{-1}$. D0 Internal Note 5902, 2009. 
[20] Higgs working group for the CDF Collaboration Tevatron New Phenomena and D0 Collaboration. Combined CDF and DZero Upper Limits on Standard Model Higgs Boson Production with up to $4.2 \mathrm{fb}^{-1}$ of Data. hep-ex/09034001, 2009.

[21] A. Djouadi, K. Kalinowski, and M. Spira. Comp. Phys. Rev., 108C:56, 1998.

[22] C. Bouchiat, J. Iliopoulos, and Ph. Meyer. Phys. Lett., 38B:519, 1972.

[23] P.W. Higgs. Phys. Rev. Lett., 13:508, 1964.

[24] F. Englert and R. Brout. Phys. Rev. Lett., 13:321, 1964.

[25] G.S. Guralnik, C.R. Hagen, and T. Kibble. Phys. Rev. Lett., 13:585, 1965.

[26] T. Kibble. Phys. Rev., 155:1554, 1967.

[27] J. Goldstone, A. Salam, and S. Weinberg. Phys. Rev., 127:965, 1962.

[28] A. Arhrib. Unitarity Constraints on Scalar Parameters of the Standard and Two Higgs Doublet Models. hep-ph/0012353, 2000.

[29] K. Riesselmann and S. Willenbrock. Phys. Rev., D55:311, 1997.

[30] C.F. Kolda and H. Murayama. JHEP, 0007:035, 2000.

[31] ALEPH Collaboration, DELPHI Collaboration, L3 Collaboration, OPAL Collaboration, and The LEP Working Group for Higgs Boson Searches. Phys. Lett. B, 565:61, 2003.

[32] M. Carena et al. Report of the Tevatron Higgs Working Group. FERMILAB-conf-00/279-T, 2000.

[33] T. Hahn, S. Heinemeyer, F. Maltoni, G. Weiglein, and S. Willenbrock. SM and MSSM Higgs Boson Production Cross Sections at the Tevatron and the LHC. hep-ph/0607308, 2006.

[34] John Conway. http://www.physics.ucdavis.edu/ conway/research/higgs/ smhiggs-tev.html.

[35] http://www.fnal.gov/pub/now/tevlum.html.

[36] D0 Collaboration. The DØ Upgrade Central Fiber Tracker Technical Design Report. D0 Note, 4154, 1999.

[37] D0 Collaboration. The Muon System of the Run II DØ Detector. Nucl. Inst. Meth., A 552:372, 2005 .

[38] D0 Offline Reconstruction Program. http://www-d0.fnal.gov/computing/algorithms/ howto/howtoreco.html.

[39] D0 RunII Event Data Model. http://www-d0.fnal.gov/software/data_model/data_ model.html\#docs.

[40] A. Khanov. HTF: histogramming method for finding tracks. The algorithm description. D0 Internal Note, 3778, 2000.

[41] G. Borissov. Ordering a Chaos or ... Technical Details of AA Tracking. http://www-do. fnal.gov/global_tracking/talks/, 2003.

[42] P.V.C. Hough. Machine Analysis of Bubble Chamber Pictures. International Conference on High Energy Accelerators and Instrumentation, 1959.

[43] H. Greenlee. The DØ Kalman Track Fit. D0 Internal Note, 4303, 2004. 
[44] A. Schwartzman and C. Tully. Primary Vertex Reconstruction by Means of Adaptive Vertex Fitting. D0 Internal Note 4918, 2005.

[45] M. Narain and A. Schwartzman. Probabilistic Vertex Selection. D0 Internal Note 4042, 2002.

[46] Y. Peters, A. Schwartzman, and M. Strauss. Certification of the Adaptive Primary Vertex in p17. D0 Internal Note 5192, 2006.

[47] F. Fleuret. The D $\varnothing$ Electron Photon Analysis Package EMAnalyze. $h t t p: / / w w w-d o$. fnal. gov/d0dist/dist/releases/p21.09.00/em_analyze/doc/EM\%Analyze_doc.ps, 1999.

[48] L. Duflot and M. Ridel. The CellNN algorithm: cell level clustering in the DØ calorimeter. Do Internal Note 3923, 2001.

[49] M. Narain. Electron Identification in the DØ Detector. DPF 92 FERMILAB-CONF-93-054E, 1992.

[50] J. Kozminski et al. Electron Likelihood in p14. D0 Internal Note 4449, 2004.

[51] E. Busato and B. Andrieu. Jet Algorithms in the D0 RunII Software: Description and User's Guide. Do Internal Note 445\%, 2004.

[52] G. Bernardi, B. Olivier, B. knuteson, and M. Strovink. NADA: A New Event by Event Hot Cell Killer. Do Internal Note 368\%, 1999.

[53] G. Bernardi and Sophie Trincaz-Duvoid. Improvement of the NADA Algorithm: Hot Cell Killing in D0 RunII Data. D0 Internal Note 405\%, 2002.

[54] U. Bassler and G. Bernardi. Towards a Coherent Treatment of Calorimetric Energies: Missing Transverse Energy, Jets, EM objects and the T42 Algorithm. D0 Internal Note 4124, 2003.

[55] J. Vlimant, U. Bassler, G. Bernardi, and S. Trincaz-Duvoid. Technical Description of the T42 Algorithm for the Calorimeter Noise Suppression. D0 Internal Note 4146, 2003.

[56] G. Bernardi, E. Busato, and J. Vlimant. Improvements from the T42 Algorithm on Calorimeter Ojects Reconstruction. D0 Internal Note 4335, 2004.

[57] L. Sawyer and A. Stone. Missing ET Reconstruction: Variable and Methods. D0 Internal Note 3957, 2003.

[58] S. Trincaz-Duvoid and P. Verdier. Missing ET Reconstruction in p17. D0 Internal Note 4474, 2004.

[59] D0 Muon ID Group. Runii d0 muon identification.

[60] C. Luo. Muon Identification and B Physics Studies at the Tevatron Collider Experiment D0. Ph. D. Thesis, Indiana University, 2003.

[61] O. Peters. Muon segmentation algorithm.

[62] D0 Muon ID Group. p20 muon certification criteria.

[63] D0 JES Group. Jet Energy Scale Determination at D0 RunII. D0 Internal Note 5382, 2007.

[64] Devaughan et al. Jet Energy Scale Determination for D0 RunIIb. D0 Internal Note 5801, 2008.

[65] N. Makovec and J. Grivaz. Shifting, Smearing and Removing Simulated Jets. Do Internal Note 4914, 2005.

[66] N. Makovec and J. Grivaz. The Relative Data-Monte Carlo Jet Energy Scale. Do Internal Note 4807, 2005. 
[67] B. Andrieu, A. Harel, H. Nogima, M. Rangel, and M. Voutilainen. Measuring Reconstruction Jet-ID Efficiencies using the tag-and-probe Method in p17. D0 Internal Note 5250, 2006.

[68] S. Callvet, P. Verdier, and E. Kajfasz. Towards Missing $E_{T}$ Certification and Unclustered Energy Studies. D0 Internal Note 4927, 2005.

[69] C. Amsler et al. (Particle Data Group). Phys. Lett., B667:1, 2008.

[70] A. Schwartzman and M. Narain. Secondary Vertex Reconstruction Using the Kalman Filter. D0 Internal Note 3908, 2001.

[71] L. Feligioni et al. Update on b-quark Jet Identification with Secondary Vertex Reconstruction Using D0RECO p14. D0 Internal Note 4414, 2004.

[72] R. Demina et al. b-tagging with Counting Signed Impact Parameter Method. D0 Internal Note 4049, 2002.

[73] D. Bloch et al. Update of the JLIP b-tagger Performance in p14/pass2 with JES 5.3. D0 Internal Note 4824, 2005.

[74] K. Hanagaki et al. Identification of b-jet by Soft Muon. D0 Internal Note 4867, 2005.

[75] M. Anastasoaie et al. Performance of the NN b-tagging Tool on p17 Data. Do Internal Note 5213, 2007.

[76] B. Clement et al. SystemD or how to get signal, backgrounds and their efficiencies with real data. Do Internal Note 4159.

[77] S. Dutt, G. Garzon, D. Lincoln, and S. Beri. p20 Taggability Studies. D0 Internal Note 5773, 2008.

[78] The DØ RunII Software Algorithms. http://www-d0.fnal.gov/computing/algorithms/.

[79] Common Samples Group. http://www-d0.fnal.gov/Run2Physics/cs/skimming/ fixPass2p170903.html.

[80] Common Samples Group. http://www-d0.fnal.gov/Run2Physics/cs/skimming/p20_ pass $2 . h t m l$.

[81] Common Samples Group. http://www-d0.fnal.gov/Run2Physics/cs/skimming/p20_ pass4.html.

[82] Common Samples Group. http://www-d0.fnal.gov/Run2Physics/cs/skimming/p20_ pass2_skims.html.

[83] S. H. Ahn et al. Accessing Triggered Luminosity Information via Flat Files. DØ Note 3969.

[84] DØ Luminosity ID group. http://www-d0.fnal.gov/phys_id/luminosity/.

[85] Begel et al. Luminosity and Performance of DØ during the First Year of Run 2. DØ Note 3973, 2003.

[86] Michael Begel, Dan Edmunds, Philipe Laurens, and Richard Partridge. DØ Luminosity in Run 2: Triggered. DØ Note 3971, 2003.

[87] P. Calfayan. ORing single-muon triggers in P17 data. DØ Note 5329.

[88] J. Pumplin, D.R. Stump, J. Huston, H.L. Lai, P. Nadolsky, and W.K. Tung. New Generation of Parton Distributions with Uncertainties from Global QCD Analysis. hep-ph/0201195, 2002.

[89] T. Sjostrand, Stephen Mrenna, and Peter Skands. PYTHIA 6.4 Physics and Manual. hepph/0603175, 2006. 
[90] M.L. Mangano, M. Moretti, F. Piccinini, R. Pittau, and A. Polosa. A Generator for Hard Multiparton Processes in Hadronic Collisions. hep-ph/0206293, 2003.

[91] E. Boos, V. Bunichev, M. Dubinin, L. Dudko, V. Ilyin, A. Kryukov, V. Edneral, V. Savrin, A. Semenov, and A. Sherstnev. CompHEP 4.4 - Automatic Computations from Lagrangians to Events. hep-ph/0403113, 2004.

[92] V. N. Gribov and L. N. Lipatov. Sov. J. Nucl. Phys., 15:438, 1972.

[93] G. Altarelli and G. Parisi. Nucl. Phys., B126:298, 1977.

[94] L. Dokshitzer. Sov Phys. JETP, 46:641, 1977.

[95] B. Andersson, G. Gustafson, G. Ingelman, and T. Sjostrand. Phys. Rep., 97:31, 1983.

[96] S. Hoche, F. Krauss, N. Lavesson, L. Lonnblad, and Mangano. Matching parton showers and matrix elements. hep-ph/0602031, 2006.

[97] E.E. Boos, V.E. Bunichev, L.V. Dudko, V.I. Savrin, and V.V. Sherstnev. Methods for simulating electroweak top-quark production events in the nlo approximation: Singletop event generator. Phys. Atom. Nucl., 69:1317, 2006.

[98] Y. Fisyak and J. Womersley. D0gstar d0 geant simulation of the total apparatus response. Do Internal Note, 3191, 1997.

[99] D0 Sim. http://www-d0.fnal.gov/computing/MonteCarlo/simulation/d0sim.html.

[100] S. Agostinelli et al. Geant4 - a simulation toolkit. Nucl. Inst. Meth., A 506:250, 2003.

[101] D0 Data Quality Coordination. http://www-do.fnal.gov//computing/data_quality/ dataqual.html.

[102] E. Barberis et al. The Matrix Method and its Error Calculation. D0 Internal Note 4564, 2004.

[103] G. Bernardi et al. Improved Limits on WH Production Using a Neural Net Algorithm with $1 \mathrm{fb}^{-1}$ of Run IIa Data. D0 Internal Note 5713, 2008.

[104] G. Bernardi et al. Search for WH Associated Production Using a Neutral Network and Matrix Element Approach with 2.7 fb $b^{-1}$ of RunII Data. D0 Internal Note 5714, 2009.

[105] J. Pumplin et al. Parton distributions and the strong coupling: Cteq6ab pdfs. JHEP, 0602:032, 2006.

[106] the Les Houches Accord PDF Interface. http://projects. hepforge. org/lhapdf/.

[107] F. Maltoni and T. Stelzer. Madevent: Automatic event generation with madgraph. JHEP, 0302:027, 2003.

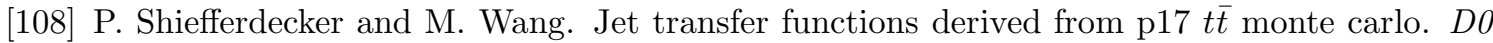
Internal Note 5136, 2006.

[109] Private communication with 1 . wang. parameterization found in the file. /work/ cole-cluedo/leiwang/wz_epmcs/p170303_sampling/wz_epmcs/src/pmcsan\%a. cpp.

[110] P. Haefner and F. Fiedler. Determination of the muon transfer function for top mass measurements. D0 Internal Note 4818, 2005.

[111] P. Haefner and F. Fiedler. Muon transfer function parameters for p17 mc. D0 Internal Note $5214,2006$. 
[112] G.P. Lepage. VEGAS: An Adaptive Multidimensional Integration Program. Cornell Laboratory of Nuclear Sciences Report CLNS-80/447, 1980.

[113] The gnu scientific library. http://www. gnu. org/software/gsl/.

[114] A. Hocker et al. TMVA (Toolkit for Multivariate Data Analysis with ROOT) Users Guide. arxiv physics/0703039, CERN-OPEN-2007-007, 2008.

[115] ROOT. http://root. cern. ch/drupal/.

[116] T. Junk. Confidence Level Computation for Combining Searches with Small Statistics. Nucl. Inst. Meth., A 434:435, 1999.

[117] W. Fisher. Calculating Limits for Combined Analyses. D0 Internal Note 4975, 2006.

[118] W. Fisher. Systematics and Limit Calculations. Do Internal Note 5309, 2006.

[119] J. Tukey. Exploratory Data Analysis. Addison-Wesley, Vol. I, Chapter 7, 1970.

[120] K. Cranmer. Kernel Estimation in High-Energy Physics. Comput. Phys. Commun. (arXiv:hep-ex/0011057v1), 136:198. 